\title{
sil
}

$4 \mathrm{H} 31992$

\section{APS Undulator and Wiggler Sources: Monte-Carlo Simulation}

by S. L. Xu, B. Lai, and P. J. Viccaro

Advanced Photon Source 
Argonne National Laboratory, with facilities in the states of Illinois and Idaho, is owned by the United States government, and operated by The University of Chicago under the provisions of a contract with the Department of Energy.

\section{DISCLAIMER}

This report was prepared as an account of work sponsored by an agency of the United States Government. Neither the United States Government nor any agency thereof, nor any of their employees, makes any warranty, express or implied, or assumes any legal liability or responsibility for the accuracy, completeness, or usefuliness of any information, apparatus, product, or process disclosed, or represents that its use would not infringe privately owned rights. Reference herein to any specific commercial product, process, or service by trade name, trademark, manufacturer, or otherwise, does not necessarily constitute or imply its endorsement, recommendation, or favoring by the United States Government or any agency thereof. The views and opinions of authors expressed herein do not necessarily state or reflect those of the United States Government or any agency thereof.

Reproduced from the best available copy.

Available to DOE and DOE contractors from the Office of Scientific and Technical Information

P.O. Box 62

Oak Ridge, TN 37831

Prices available from (615) 576-8401, FTS 626-8401

Available to the public from the

National Technical Information Service

U.S. Department of Commerce

528.5 Port Royal Road

Springfield, VA 22161 
Distribution Category: Atomic, Molecular, and Chemical Physics (UC-411)

ARGONNE NATIONAL LABORATORY

9700 South Cass Avenue

Argonne, Mlinois 60439
ANL/APS/TB--1

DE92 011209

ANL/APS/TB-1

\title{
APS Undulator and Wiggler Sources: Monte-Carlo Simulation
}

\author{
by S. L. Xu, B. Lai, and P. J. Viccaro \\ Experimental Facilities Division \\ Advanced Photon Source
}

February 1992

DISTRIBUTION OF THIS DOCUMENT IS UNLUMITED

work sponsored by

U.S. DEPARTMENT OF ENERGY

Office of Energy Research 


\section{INTRODUCTION}

Standard insertion devices will be provided to each sector by the Advarrced Photon Source. It is important to define the radiation characteristics of these general purpose devices. In this document, results of Monte-Carlo simulation are presented. These results, based on the SHADOW program, ${ }^{1}$ include the APS Undulator A (UA), Wiggler A (WA), and Wiggler B (WB).

In an undulator, the energy tuning is accomplished by changing the magnetic gap opening. Fig. 1 shows the first harmonic energy and the deflection parameter $\mathrm{K}$, as a function of the magnetic gap size for Undulator $A$. Note that by varying the magnetic gap, the first harmonic energy may be tuned from $4.5 \mathrm{keV}$ to $13.5 \mathrm{keV}$, which corresponds to a change in $\mathrm{K}$ from 2.1 to 0.3 . The brilliance of the first and the third harmonic were calculated for this range of $\mathrm{K}$ values, and the spectra are displayed in Fig. 2. Very high brilliance on the order of $10^{18}$ is generally obtained from the iirst harmonic, while the third harmonic extends the spectral range up to $40 \mathrm{keV}$. The parameters of Undulator A used for this calculation are listed in Table 1, and the energies of the first 3 harmonics computed using:

$$
\mathrm{E}=\frac{\mathrm{nhc} 2 \gamma^{2}}{\lambda_{\mathrm{u}}\left(1+\mathrm{K}^{2} / 2\right)} \quad \mathrm{n}=1,2,3
$$

are listed in Table 2.

To adequately handle and make efficient use of this unprecedented brilliance in the hard $x$-rays, detailed information about its spatial distribution is needed. For this purpose, the angular

B. Lai and F. Cerrina, Nucl. Instr. and Meth. A246, 337 (1986); B. Lai, K. Chapman and F. Cerrina, Nucl. Instr. and Meth. A 266, 544 (1988). 
distribution of $\mathrm{x}$-rays produced by Undulator $\mathrm{A}$ has been simulated here (see Figs. UA1 - UA57) for $\mathrm{K}$ ranging from 2.1 to 0.3 in steps of 0.1 . For each $\mathrm{K}$, the radiation pattern in angular space $\left(x^{\prime}, y^{\prime}\right)$ was modeled at the first 3 harmonic energies using an undulator modeling method described previously. ${ }^{2}$ In each figure, the APS beam emittance case is shown together with the limiting case of zero emittance, so that contribution from higher harmonics and effects of emittance broadening may be easily identified. Note that in most cases, the central cone is dominated by the beam divergence. These angular plots, when multiplied by the distance from the source, represent the spatial flux pattern obtained in the far-field regime. For the near-field region, the spatial distribution is further broadened by the finite source size in $x$ and $y$ (besides the zeroemittance pattern is more complex). ${ }^{3} \mathrm{~A}$ program to take into account the near-field effects is being developed at APS. Each diagram is generated with 1000 rays and is displayed over an angular limit of 1 mrad by $1 \mathrm{mrad}$ in $x^{\prime}$ and $y^{\prime}$. Because a fixed number of rays (1000) is always used in creating the angular plots, they do not show the relative intensity variation between the harmonics. That information is contained in Fig. 2.

In the second part, similar angular plots for the two wigglers are presented (Figs. WA1 - WA8 and WB1 - WB8). Wiggler A (WA), with critical energy of $32.6 \mathrm{keV}$, is optimized for the high-energy region and will extend the spectral range to $200 \mathrm{keV}$ and above (Fig. 3). Wiggler $B(W B)$, on the other hand, is designed for the lowenergy region with $E_{c}=9.8 \mathrm{keV}$ (Fig. 4). Because the radiation from a wiggler is more spread out in the horizontal direction $x^{\prime}$, all the

2 K. Chapman, B. Lai, F. Cerrina and P. J. Viccaro, Nucl. Instr. and Meth. A 283, 88 (1989).

3 See for instance R. P. Walker, Nucl. Instr. and Meth. A267, 537 (1988). 
angular diagrams are plotted over a wider limit of $2.5 \mathrm{mrad}$ by 2.5 mrad. The actual extent of the ray distribution is determined by $\mathrm{K} / \gamma$. Thus, since WA has larger $K$ than $W B$, the photons are distributed over $2 \mathrm{mrad}$ for WA versus $1 \mathrm{mrad}$ for WB. As for the spread in the vertical direction $y^{\prime}$, note that the photons are confined within smaller and smaller angles as the photon energy increases, which is similar to bending magnet radiation. The parameters used in the wiggler calculation are listed in Table 3. 


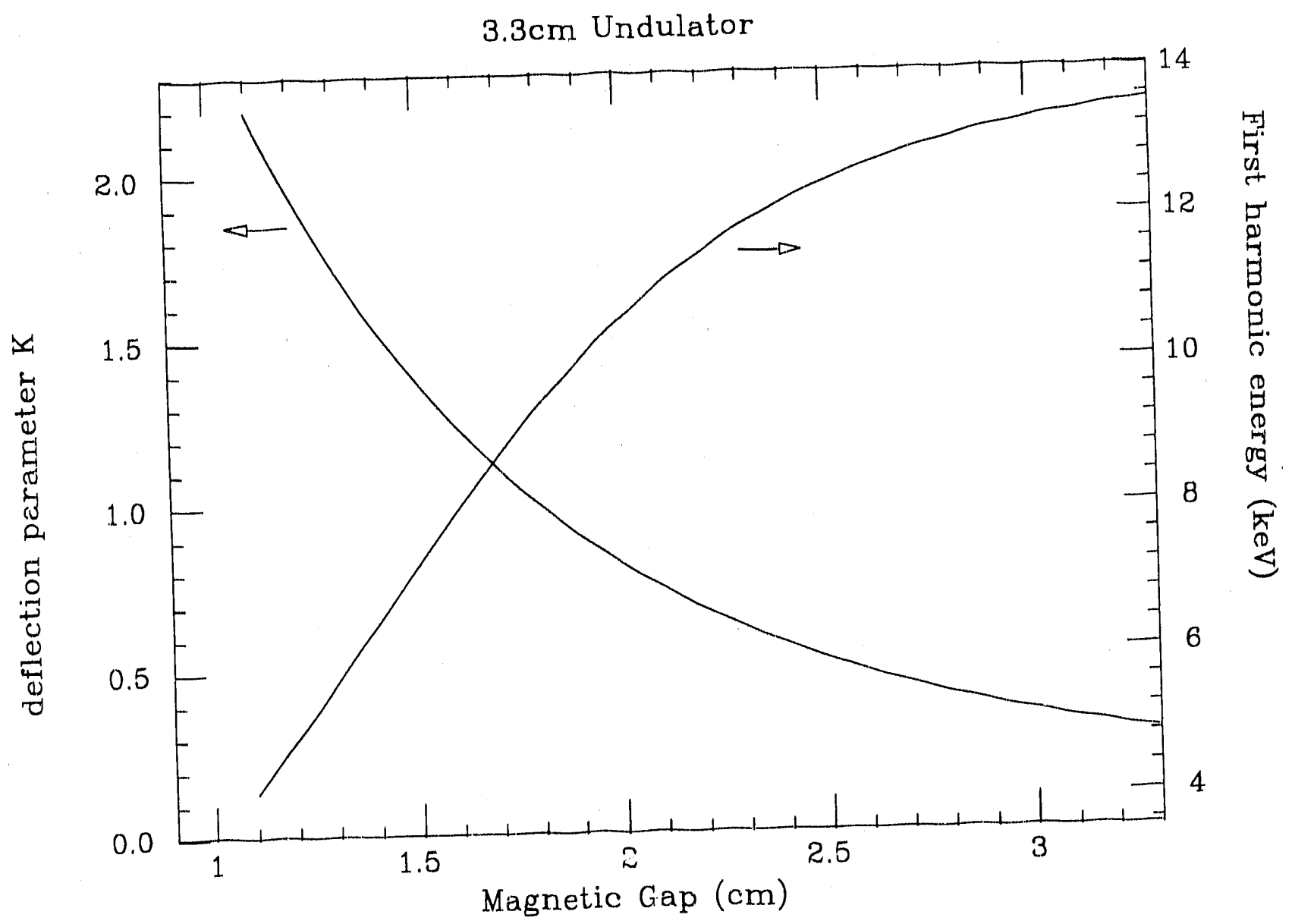

Fig. 1 First harmonic energy and deflection parameter $K$ as a function of the magnetic gap for Undulator A. 


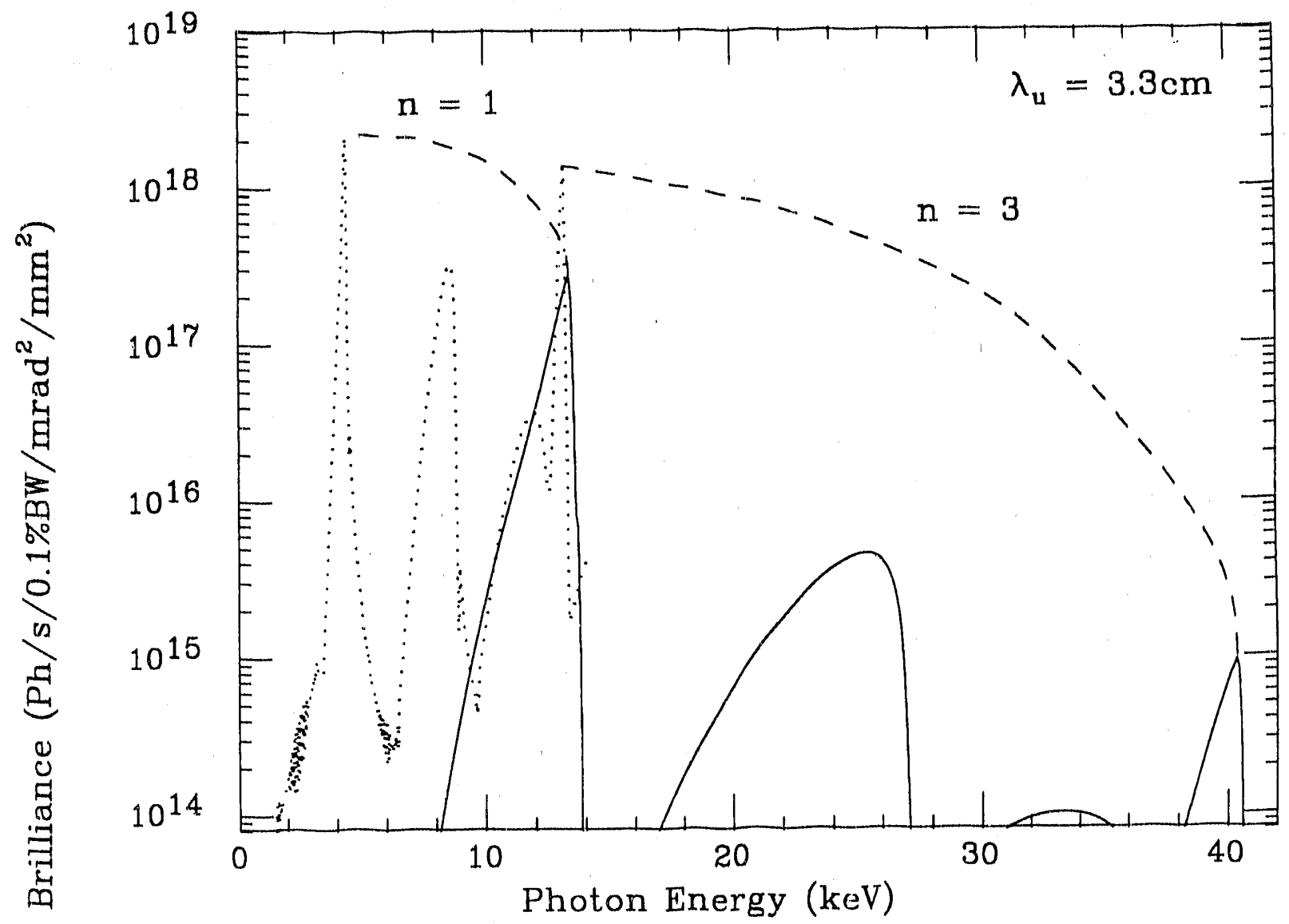

Fig. 2 On-axis brilliance of Undulator A. The spectral distribution of the first 3 harmonics are shown for the case of $\mathrm{K}=0.3$ (solid line) and $K=2.1$ (dotted line). The overall envelope of the first and the third harmonic for the $\mathrm{K}$-values in between are showed in dashed lines. 


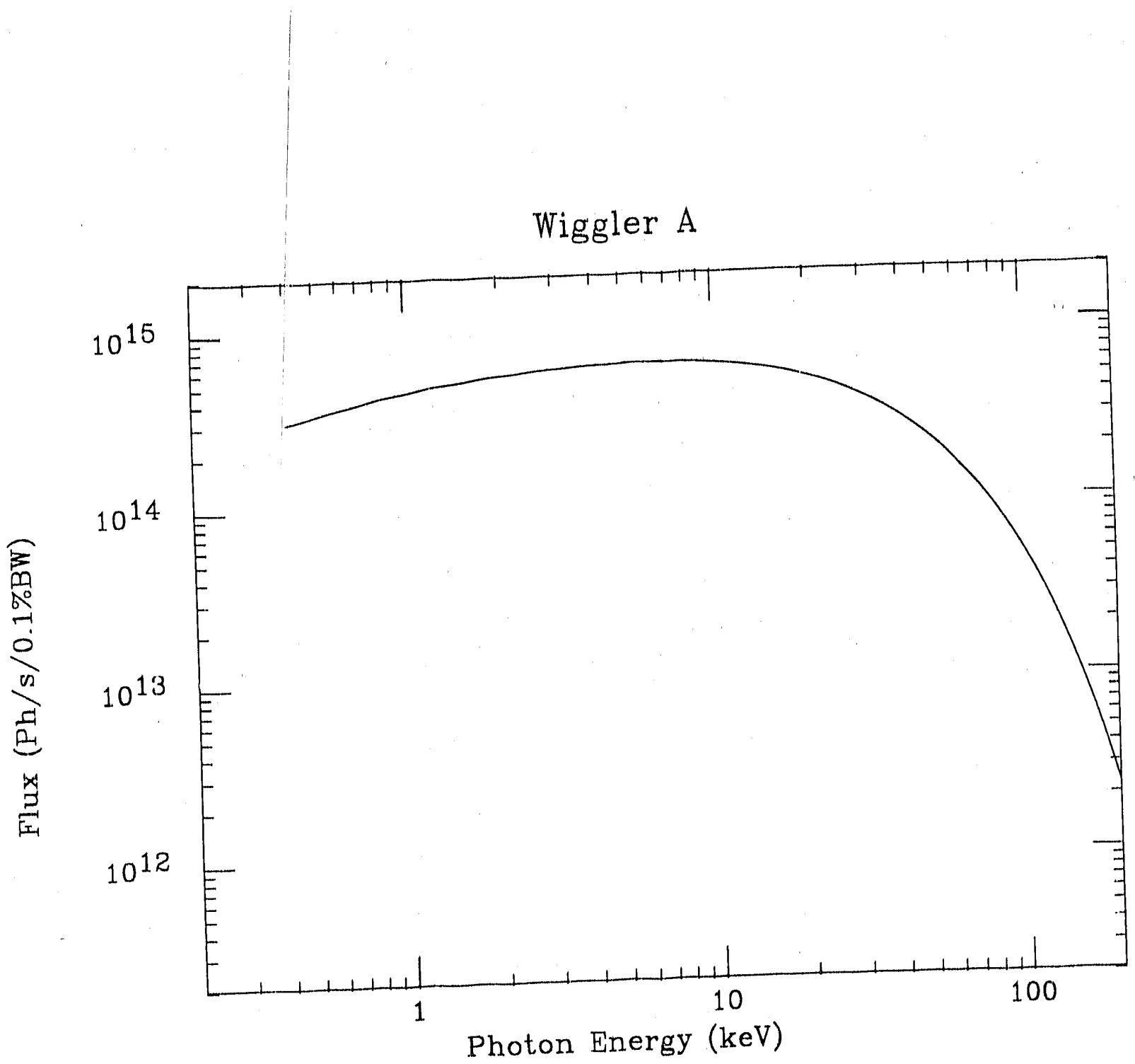

Fig. 3 Angle integrated flux for Wiggler A. 
Wiggler $B$

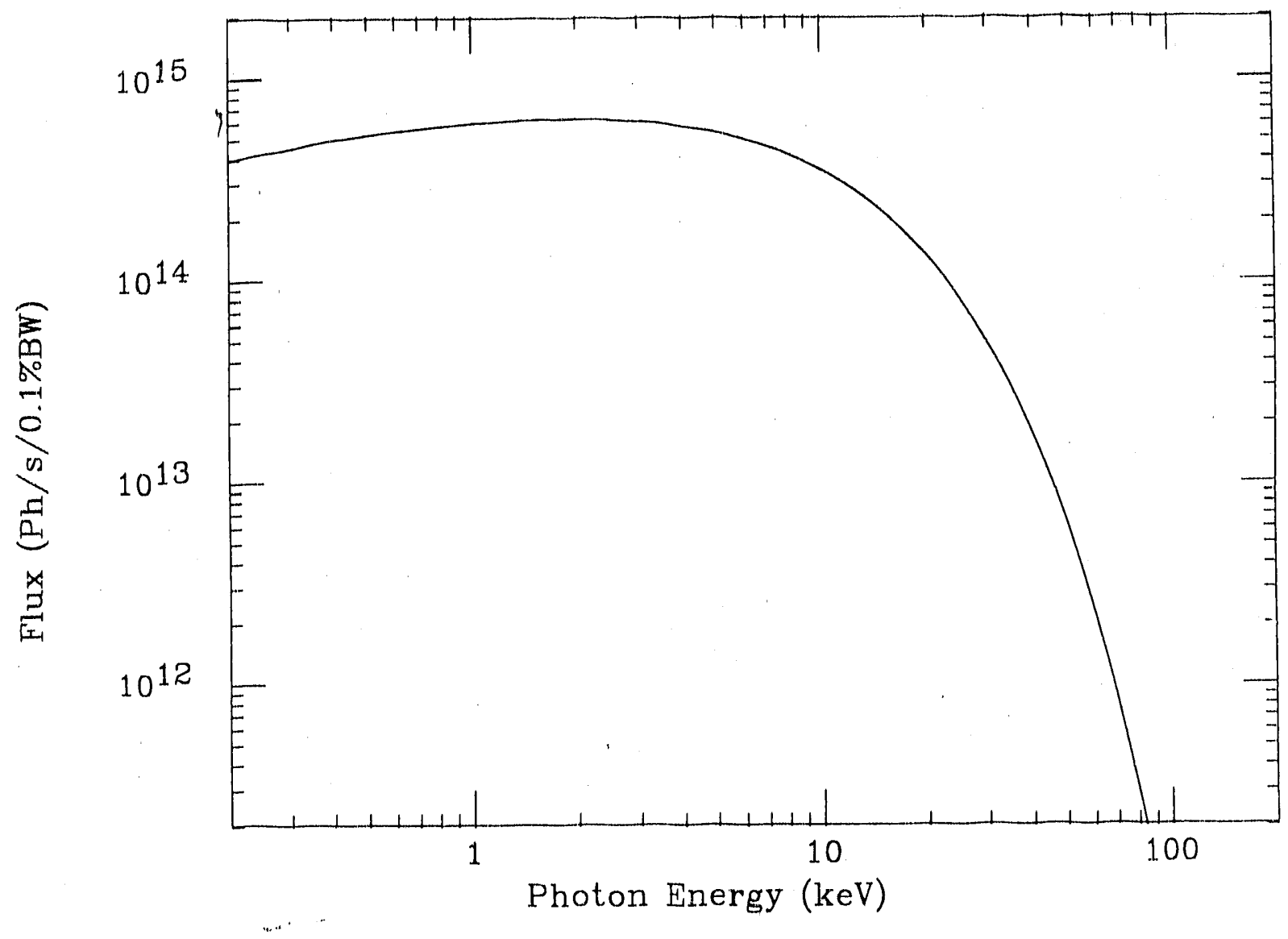

Fig. 4 Angle integrated flux for Wiggler B. 
Table 1. Parameters of Undulator A used in calculation

\begin{tabular}{|c|c|}
\hline$\lambda_{0}$ & $3.3 \mathrm{~cm}$ \\
\hline $\mathrm{K}$ & $0.3-2.1$ \\
\hline $\mathrm{N}$ & $70 \mathrm{periods}$ \\
\hline $\mathrm{E}_{\mathrm{b}}$ & $7.0 \mathrm{GeV}$ \\
\hline $\mathrm{I}_{\mathrm{b}}$ & $100 \mathrm{~mA}$ \\
\hline$\sigma_{\mathrm{x}}$ & $0.308 \mathrm{~mm}$ \\
\hline$\sigma_{\mathrm{y}}$ & $0.085 \mathrm{~mm}$ \\
\hline$\sigma_{x^{\prime}}$ & $24 \mu \mathrm{rad}$ \\
\hline$\sigma_{y^{\prime}}$ & $9 \mu \mathrm{rad}$ \\
\hline$\Delta \mathrm{E} / \mathrm{E}$ & $0.1 \% \mathrm{Bandwidth}$ \\
\hline Maximum angle & $0.3 \mathrm{mrad}$ \\
\hline
\end{tabular}

\begin{tabular}{|c|l|c|}
\hline \multirow{2}{*}{$\begin{array}{c}\text { Beam } \\
\text { Emittance }\end{array}$} & UAS-0 & zero \\
\cline { 2 - 3 } & UAS-1 & $\varepsilon_{x}=7.3, \varepsilon_{y}=0.73 \mathrm{~nm}-\mathrm{rad}$ \\
\hline
\end{tabular}


Table 2. Energy of the first 3 harmonics for Undulator A

\begin{tabular}{|c|c|c|c|}
\hline $\mathrm{K}=$ & $1^{\text {st }}$ harmonic & $2^{\text {nd }}$ harmonic & $3^{\text {rd }}$ harmonic \\
\hline 2.1 & 4.40 & 8.80 & 13.20 \\
2.0 & 4.70 & 9.40 & 14.10 \\
1.9 & 5.03 & 10.06 & 15.08 \\
1.8 & 5.38 & 10.77 & 16.15 \\
1.7 & 5.77 & 11.54 & 17.30 \\
1.6 & 6.19 & 12.37 & 18.56 \\
1.5 & 6.64 & 13.27 & 19.91 \\
1.4 & 7.12 & 14.24 & 21.37 \\
1.3 & 7.64 & 15.29 & 22.93 \\
1.2 & 8.20 & 16.40 & 24.60 \\
1.1 & 8.79 & 17.57 & 26.36 \\
1.0 & 9.40 & 18.80 & 28.20 \\
0.9 & 10.04 & 20.07 & 30.11 \\
0.8 & 10.68 & 21.37 & 32.05 \\
0.7 & 11.33 & 22.65 & 33.98 \\
0.6 & 11.95 & 23.90 & 35.85 \\
0.5 & 12.54 & 25.07 & 37.61 \\
0.4 & 13.06 & 26.12 & 39.17 \\
0.3 & 13.50 & 26.99 & 40.49 \\
\hline
\end{tabular}


Table 3. Parameters of Wiggler A and Wiggler B used in calculation

\begin{tabular}{|c|c|c|}
\hline & WA & WB \\
\hline$\lambda_{0}$ & $15 \mathrm{~cm}$ & $25 \mathrm{~cm}$ \\
\hline $\mathrm{K}$ & 14 & 7 \\
\hline $\mathrm{N}$ (periods) & 10 & 20 \\
\hline$E_{c}$ & $32.57 \mathrm{keV}$ & $9.77 \mathrm{keV}$ \\
\hline $2 \theta$ & 2 rnrad & $1 \mathrm{mrad}$ \\
\hline $\mathrm{E}_{\mathrm{b}}$ & \multicolumn{2}{|c|}{$7 \mathrm{GeV}$} \\
\hline$I_{b}$ & \multicolumn{2}{|c|}{$100 \mathrm{~mA}$} \\
\hline$\Delta \mathrm{E} / \mathrm{E}$ & \multicolumn{2}{|c|}{$0.1 \%$ Bandwidth } \\
\hline$\sigma_{x}$ & \multicolumn{2}{|c|}{$0.308 \mathrm{~mm}$} \\
\hline$\sigma_{y}$ & \multicolumn{2}{|c|}{$0.085 \mathrm{~mm}$} \\
\hline$\sigma_{x^{\prime}}$ & \multicolumn{2}{|c|}{$24 \mu \mathrm{rad}$} \\
\hline$\sigma_{y^{\prime}}$ & \multicolumn{2}{|c|}{$9 \mu \mathrm{rad}$} \\
\hline$\varepsilon_{\mathrm{x}}$ & \multicolumn{2}{|c|}{$7.3 \mathrm{~nm}-\mathrm{rad}$} \\
\hline$\varepsilon_{\mathrm{y}}$ & \multicolumn{2}{|c|}{0.73 nm-rad } \\
\hline
\end{tabular}


-

잉

C 


\section{APS UNDULATOR A}

\section{RAY-TRACING RESULTS}


USER \$DISK:[XUS.SHADOW]BEGIN.DAT;7
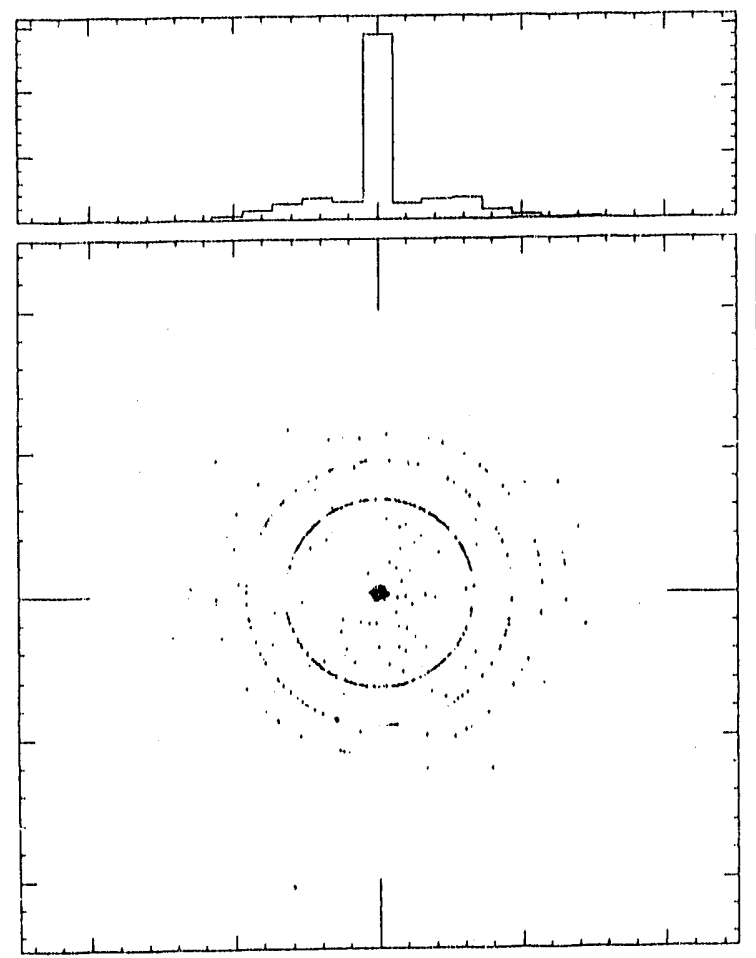

USER \&DISK:[XUS.SHADOW]BEGIN.DAT;8
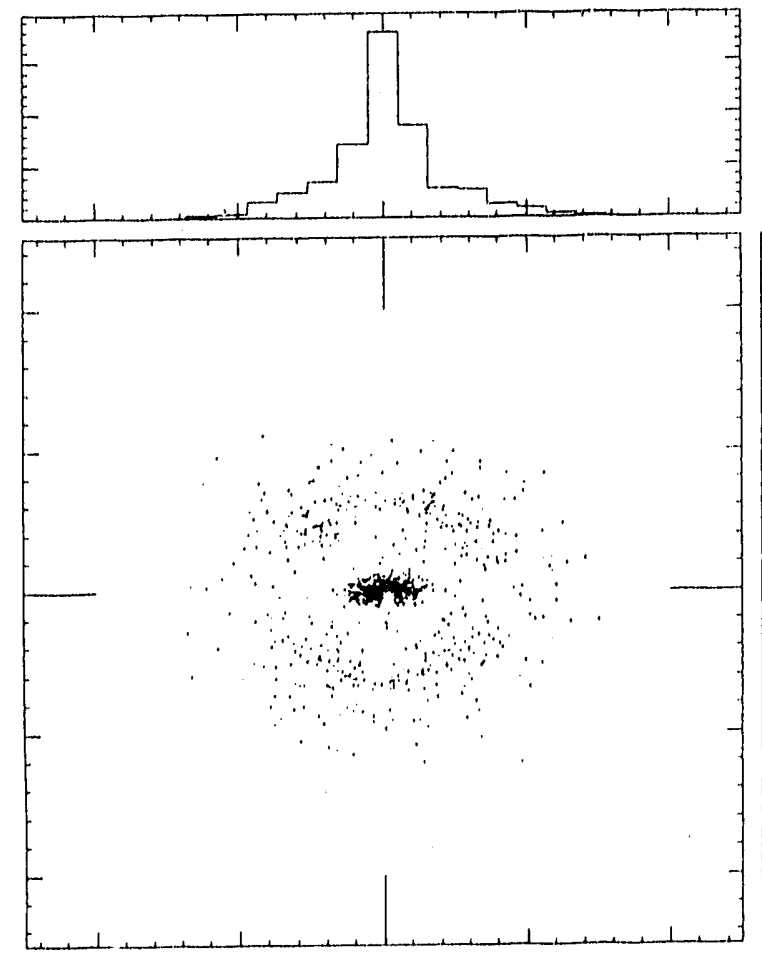

10)-APR+1991 08:51

UAS O $k=2.1 F E=4.4 k \theta$

V

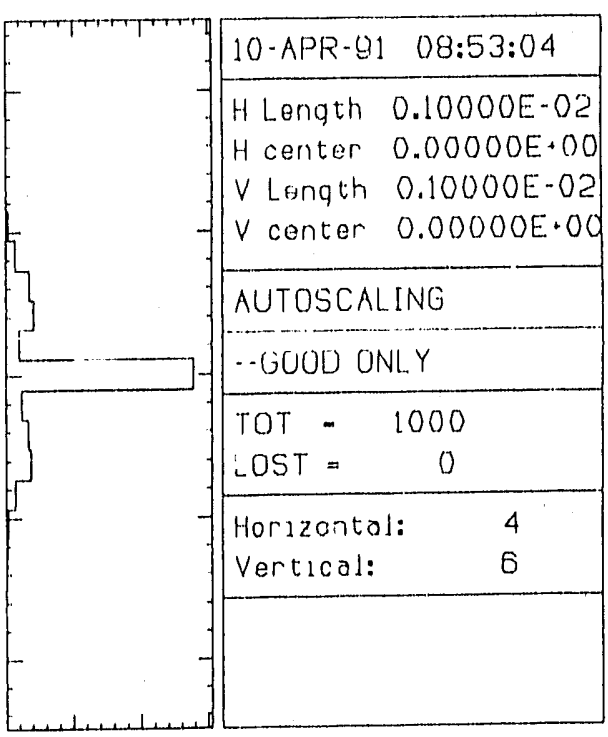

$10 \cdot A P R-199108: 55$

UAS-1 $K=2.1 F \quad E=4.4 \mathrm{ke}$

V

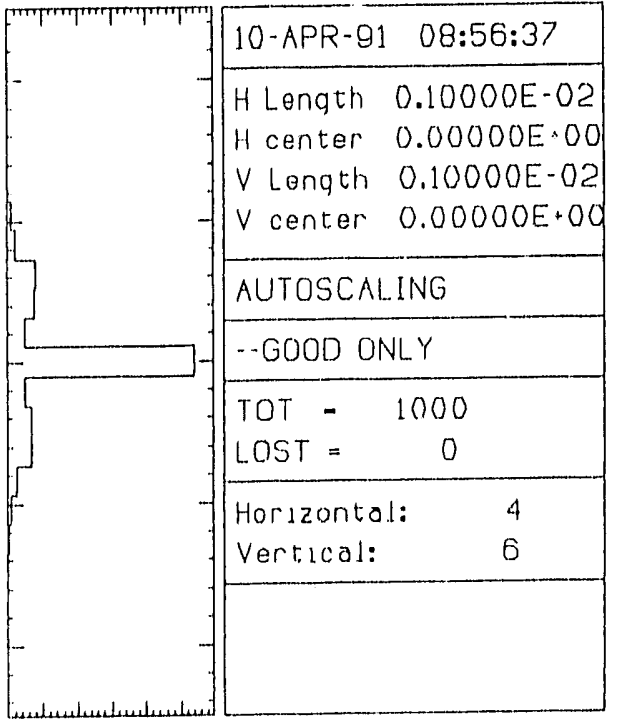

Fig. UA-1. The angular pattern at the first harmonic $(\mathrm{h} v=4.40 \mathrm{keV})$ for $K=2.1$. The zero-emittance case is shown on the top (UAS-0), while the bottom part shows the case for APS emittance (UAS-1). The limits for each plot are $1 \mathrm{mrad} \times 1 \mathrm{mrad}$. 


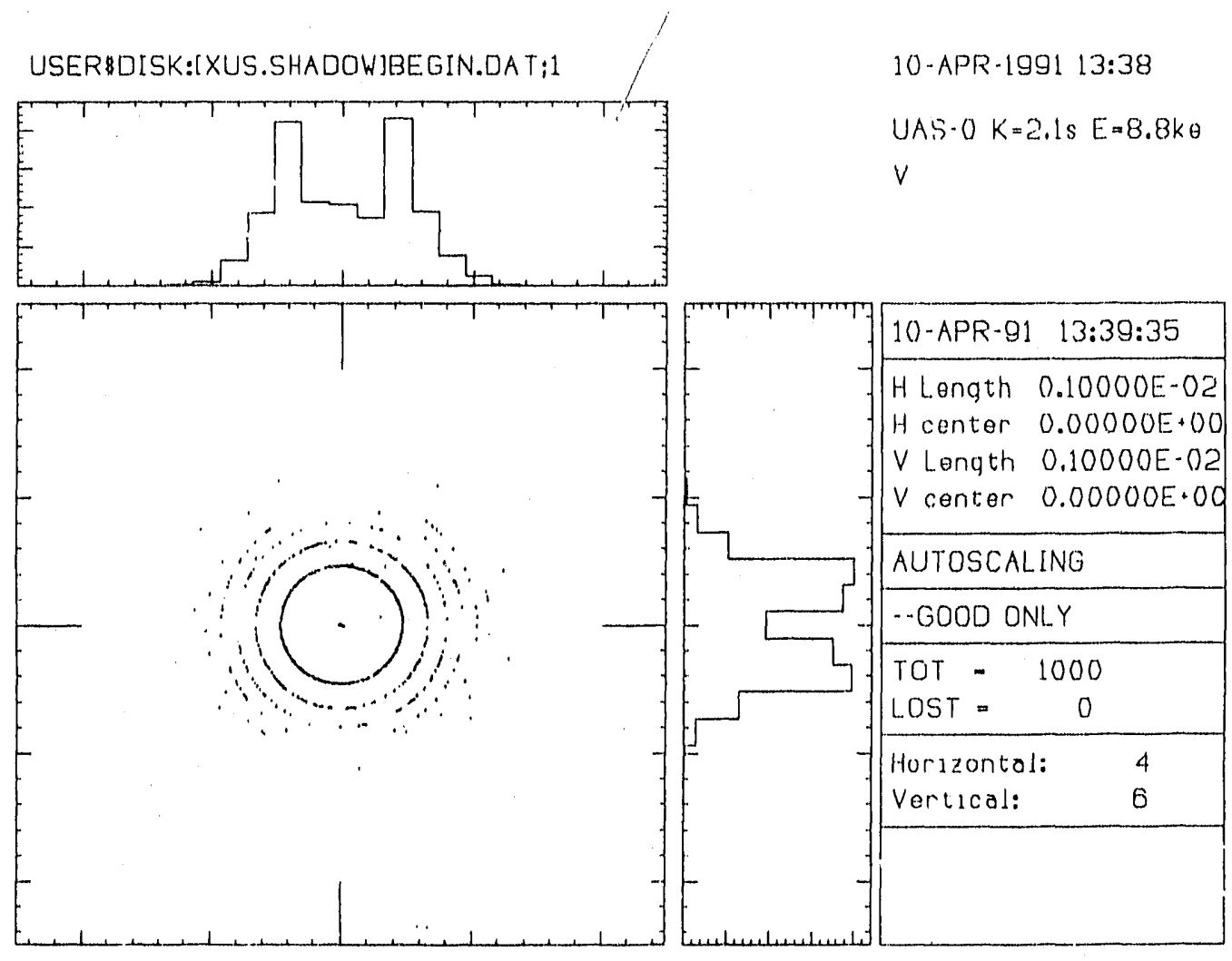

USER SDISK:[XUS.SHADOW]BEGIN.DAT;2
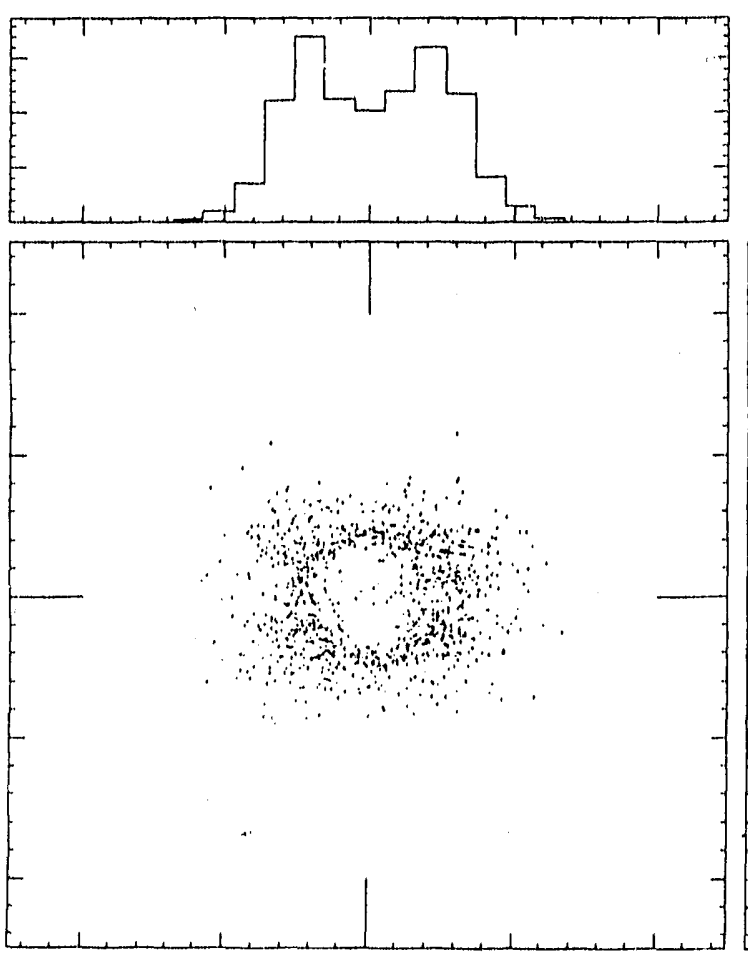

Fig. UA-2. $\quad \mathrm{K}=2.1$, second harmonic.

10-APR-1991 1.3:41

UAS $-1 K=2.1 \mathrm{~s} E=8.8 \mathrm{~kg}$

$\checkmark$

$\checkmark$ center $0.00000 \mathrm{E}$.0

AUTOSCALING

-GOOD ONLY

TOT - 1000

LOST $=0$

Horizontal: 4

Vertical: $\quad 6$

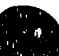


USERBDISK:[XUS.SHADDW]BEGIN.DAT;3
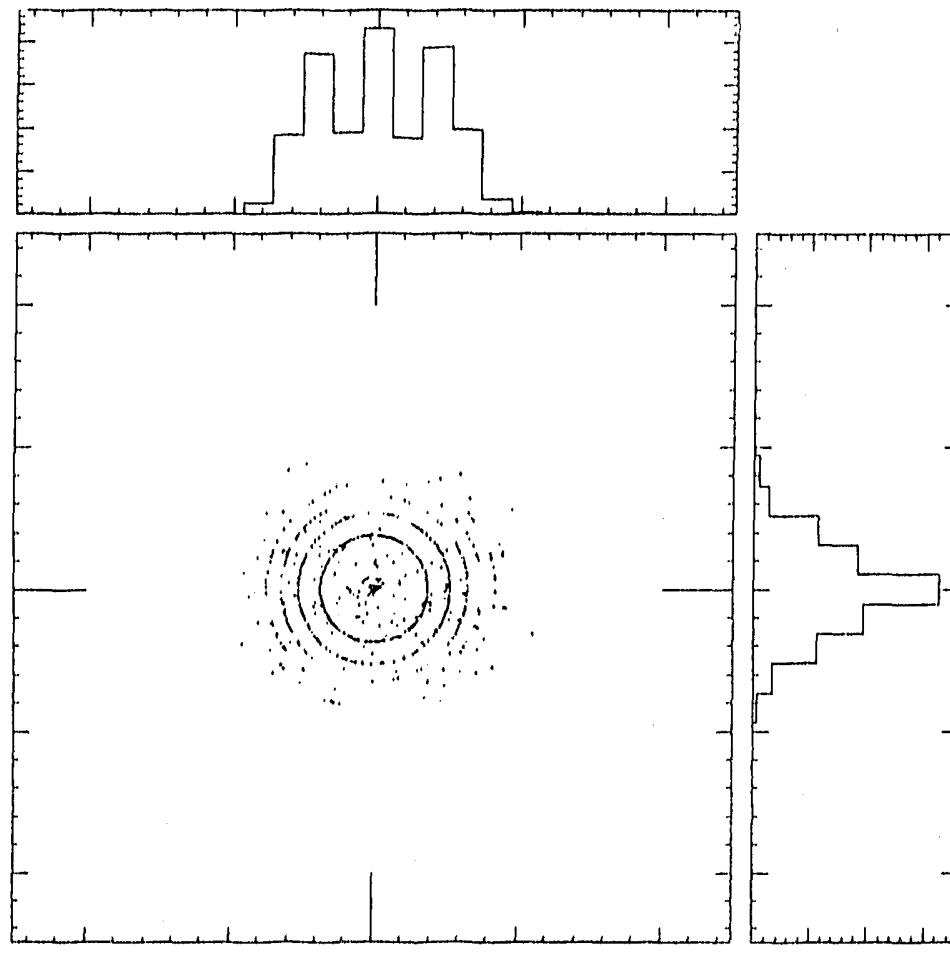

USER\&DISK:IXUS.SHADOWIBEGIN.DAT;4
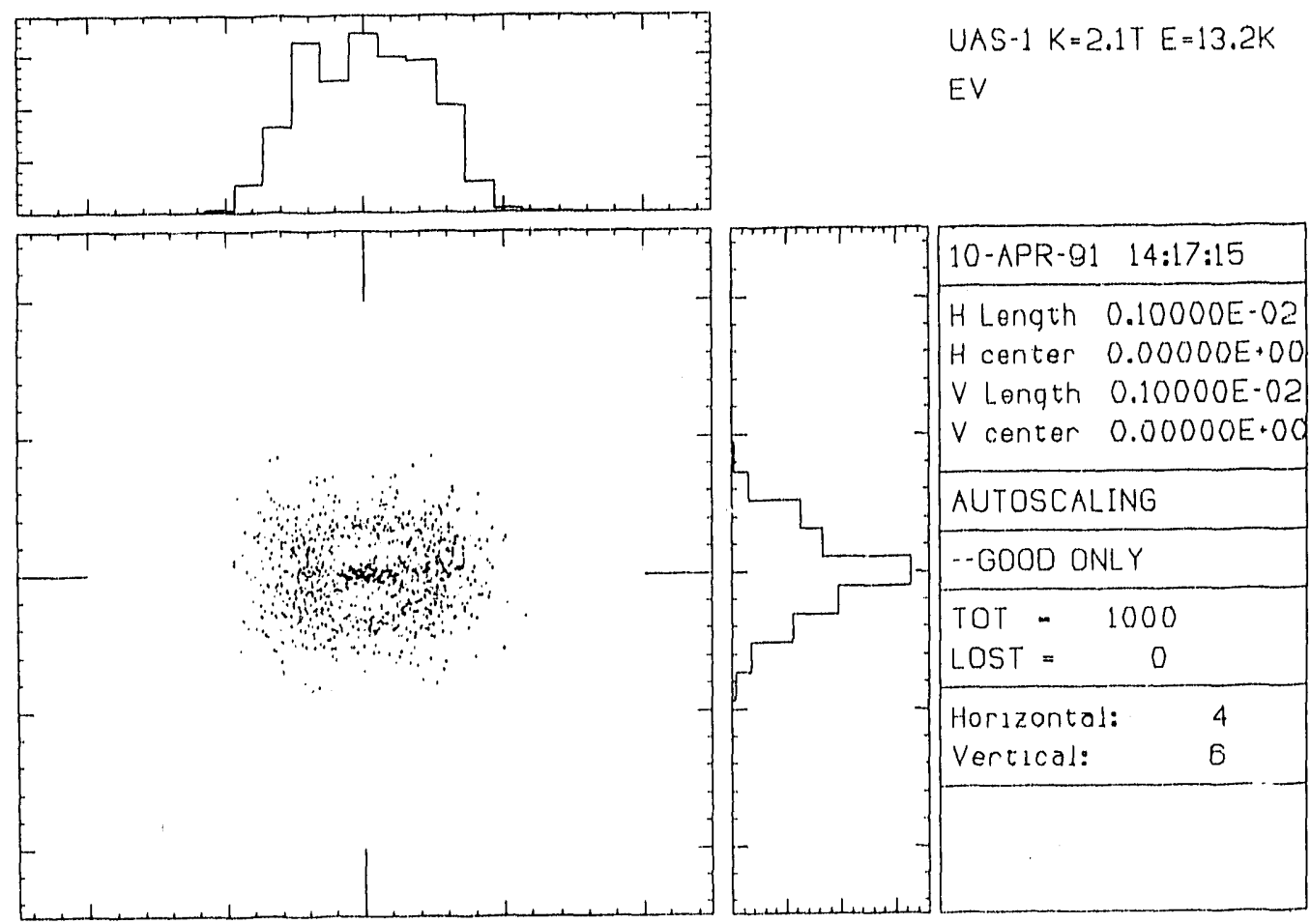

Fig. UA-3. $\quad \mathrm{K}=2.1$, third harmonic.
10-APR-1991 14:05

UAS-O $K=2.1 T E=13.2 \mathrm{k}$

${ }_{\theta} \mathrm{V}$

10-APR-91 14:06:35

$H$ Length $0.10000 E-02$

H center $0.00000 E+00$

$V$ Length $0.10000 E-02$

$V$ center $0.00000 E \cdot 00$

AUTOSCALING

--GOOD ONLY

TOT - 1000

LOST $=0$

Horizontal: 4

10-APR-1991 14:13

UAS-1 $K=2.1 \mathrm{~T} E=13.2 \mathrm{~K}$

EV
Vertical: 6 
USER OUISK:LXUS.SHADOWIBEGIN.DAT:7
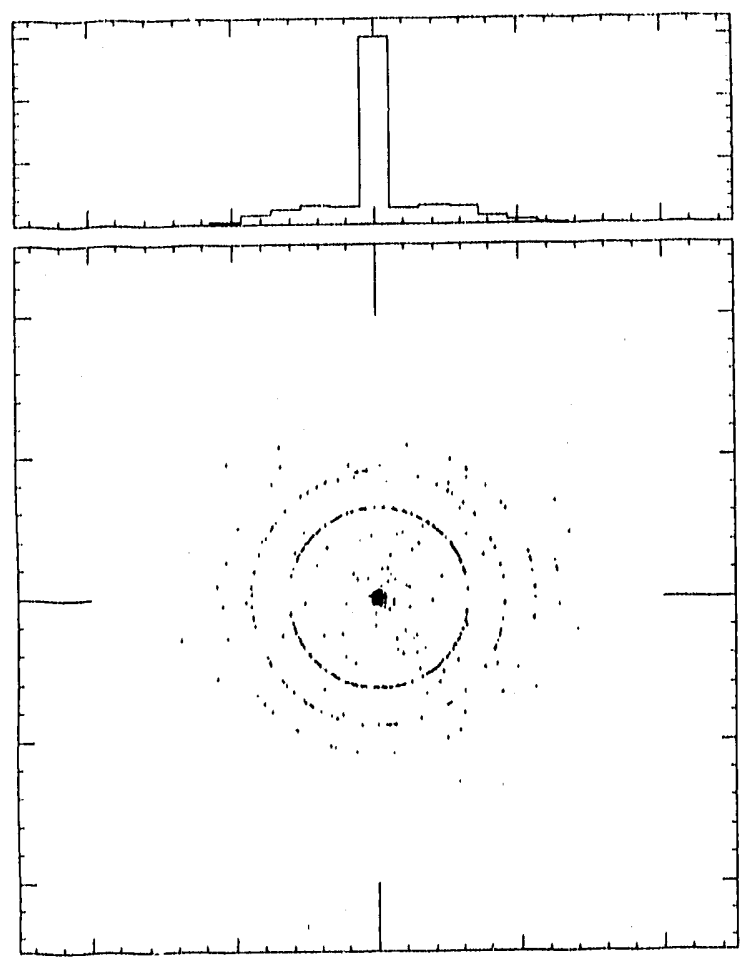

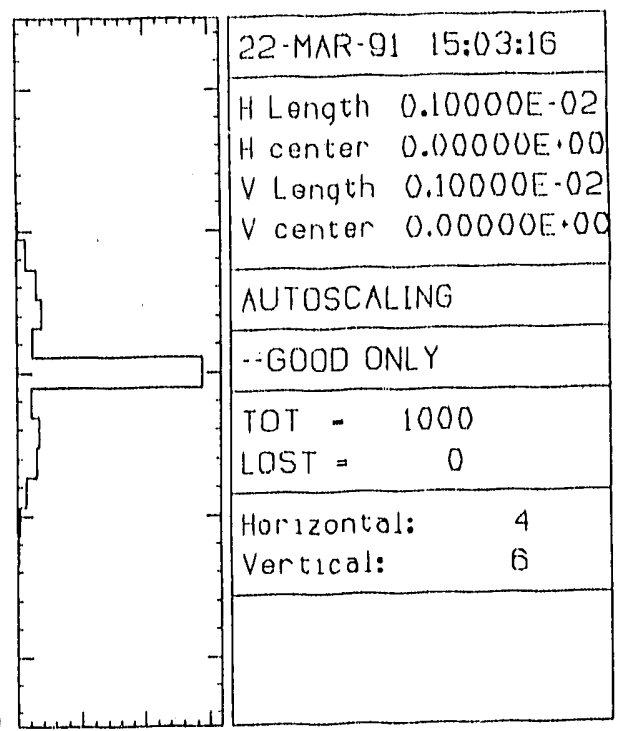

22-MAR-1991 15:01

UAS-O $k^{\prime}=2.0 \quad E=4.7 \mathrm{kgV}$
USER \#DISK:[XUS.SHADOW]BEGIN.DAT:8
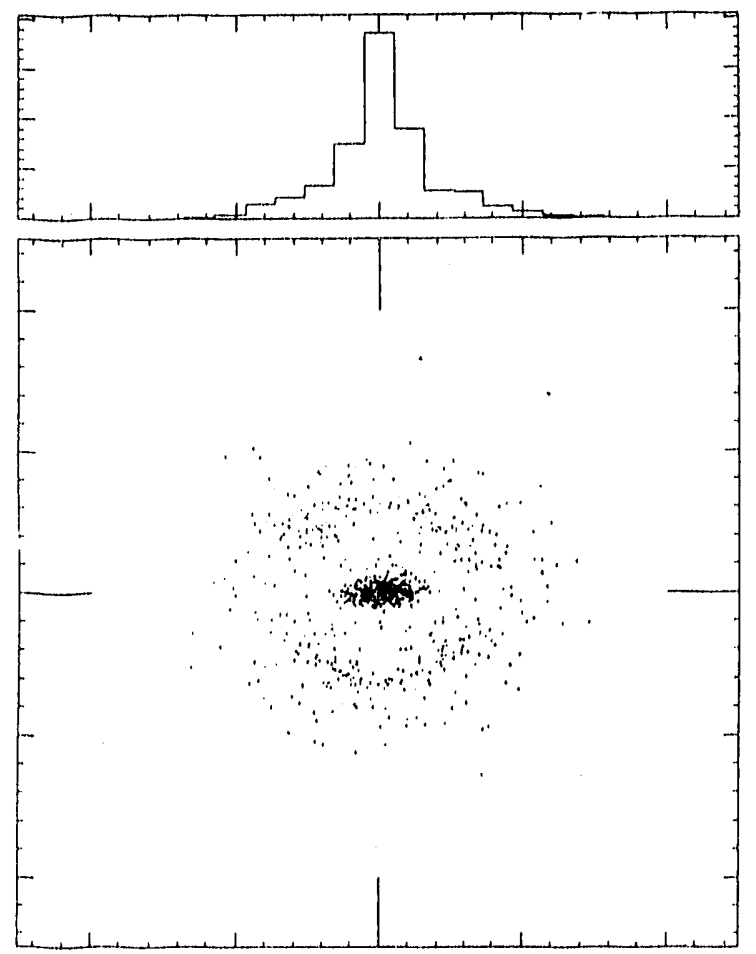

22-MAR-19011 15:07

UAS-1 $K=2.0 E=4.7 \mathrm{kOV}$

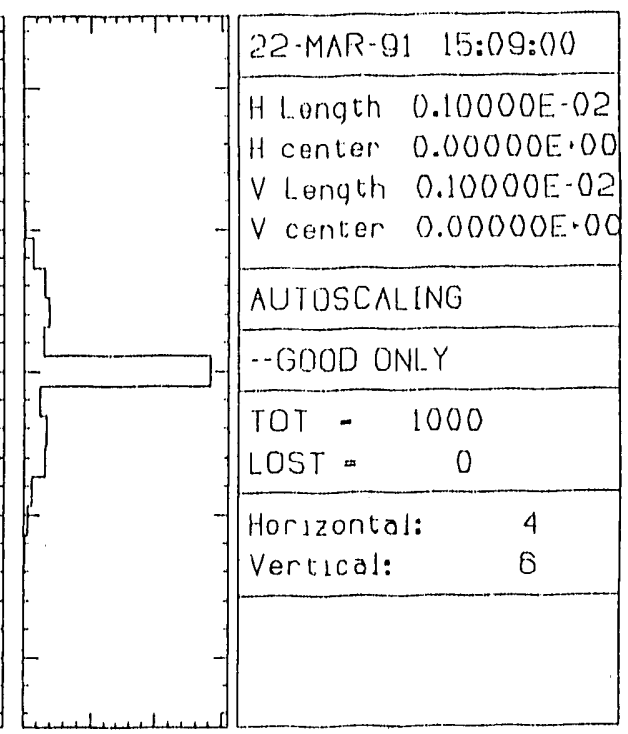

Fig. UA-4. $\quad K=2.0$, first harmonic. 

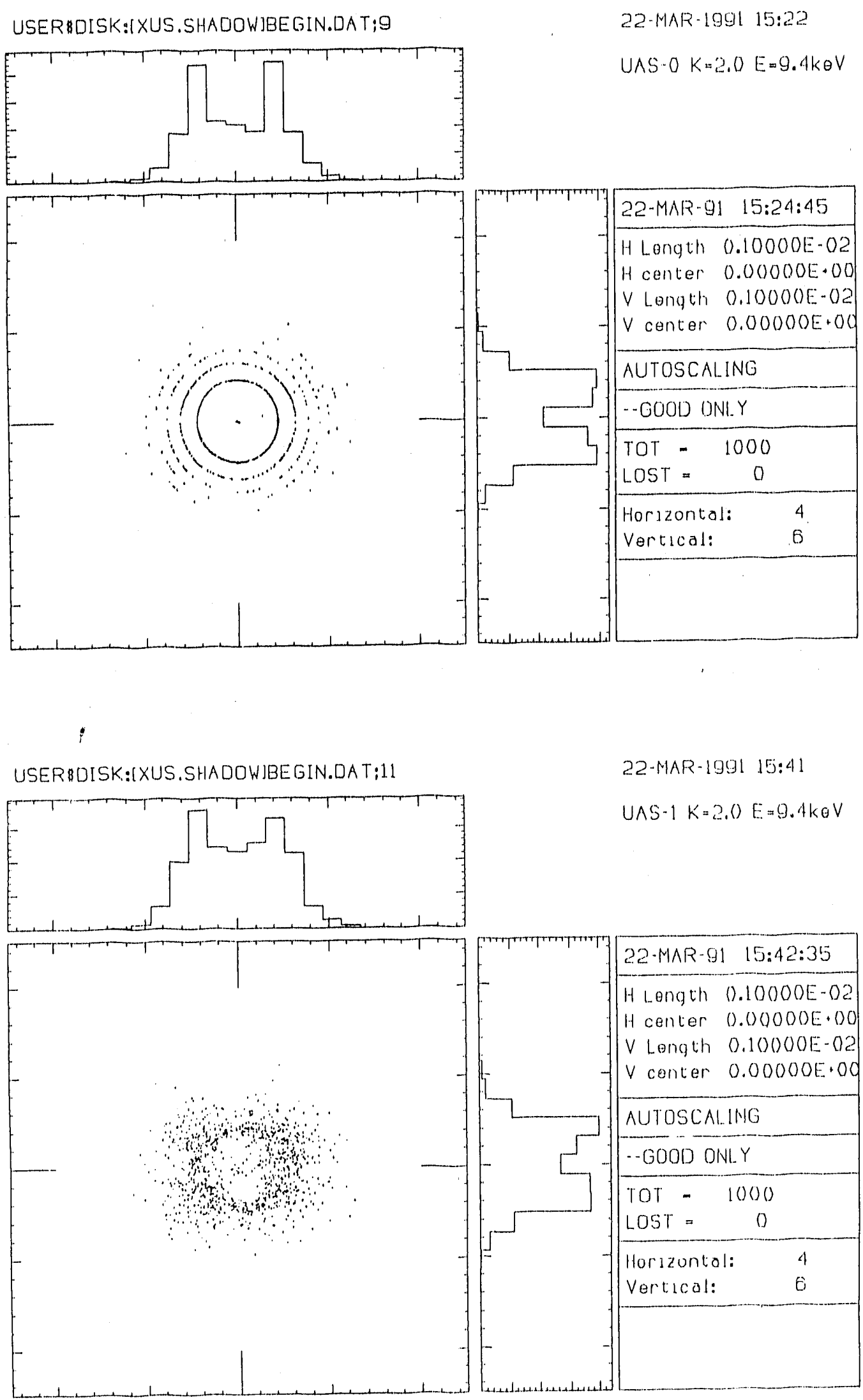

Fig. UA-5. $\quad \mathrm{K}=2.0$, second harmonic. 
USERBLISK:IXIJS.SIIAOOWJBEGIN.DATIL
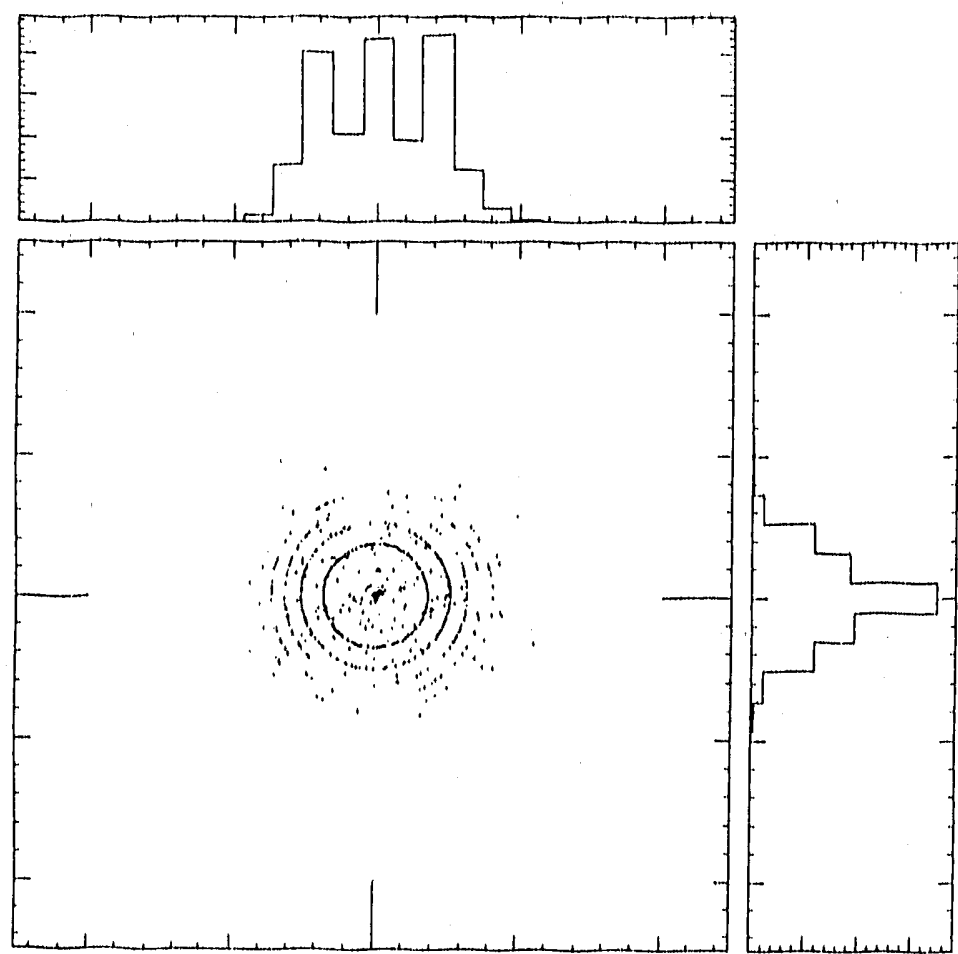

Q2-MAR-10911 16:16

$U \wedge S-() K=2.0) E=14.1 k \theta$

$\checkmark$
22.-MAR-91 10:18:30

H Longth $0.10000 E-02$

H center $0.00000 E+00$

$V$ l.ength $0.10000 \mathrm{E}-02$

$\checkmark$ center $0.00000 E+00$

AUTOSCALING

-GOOD ONLY'

TOT - 1000

LOST = 0

Horizontal: 4

Vertical: $\quad 6$

22-MAP-1991 16:02

UAS-1 $K=2.0 E=1.1 .1 k \theta$

$\checkmark$

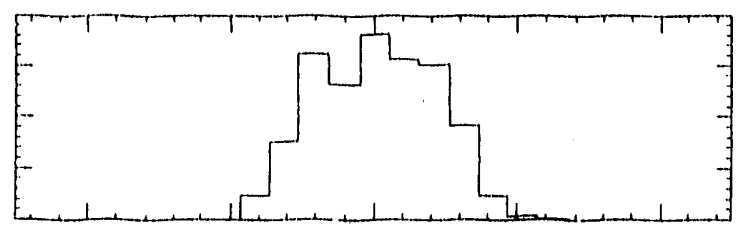

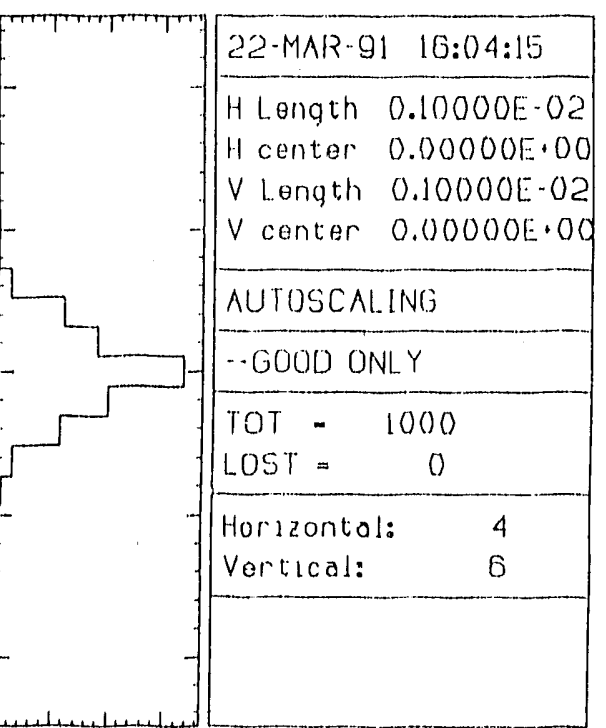

Fig. UA-6. $\quad \mathrm{K}=2.0$, third harmonic. 
LSERBDISK:IXUSIBEGIN.DAT:1
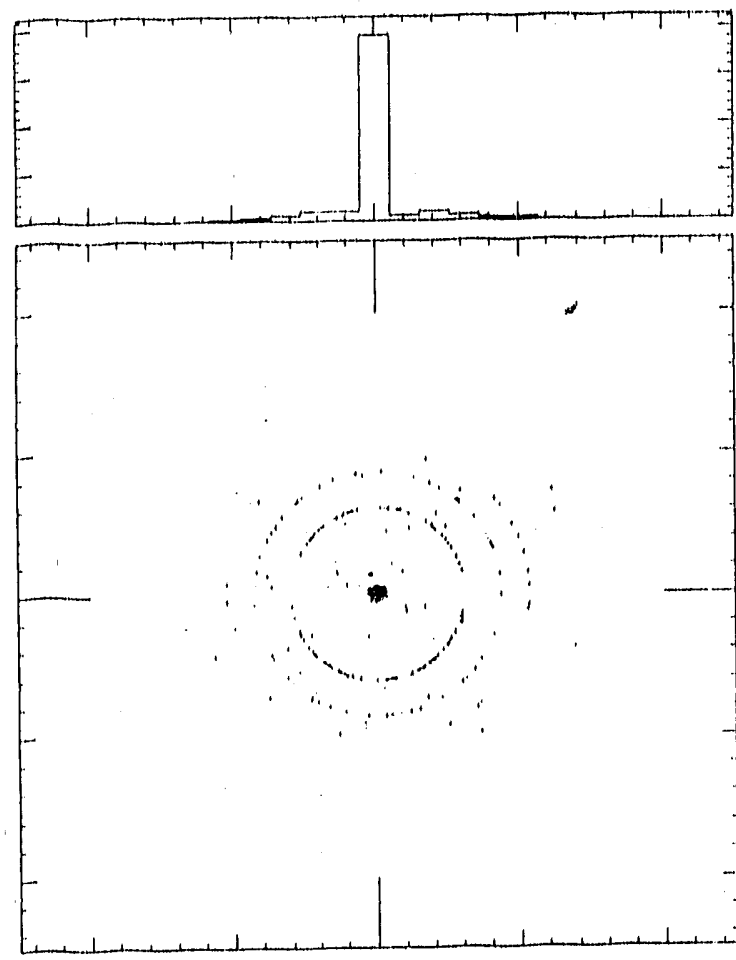

USERADISK:LXUSIBEGIN.DAT:3
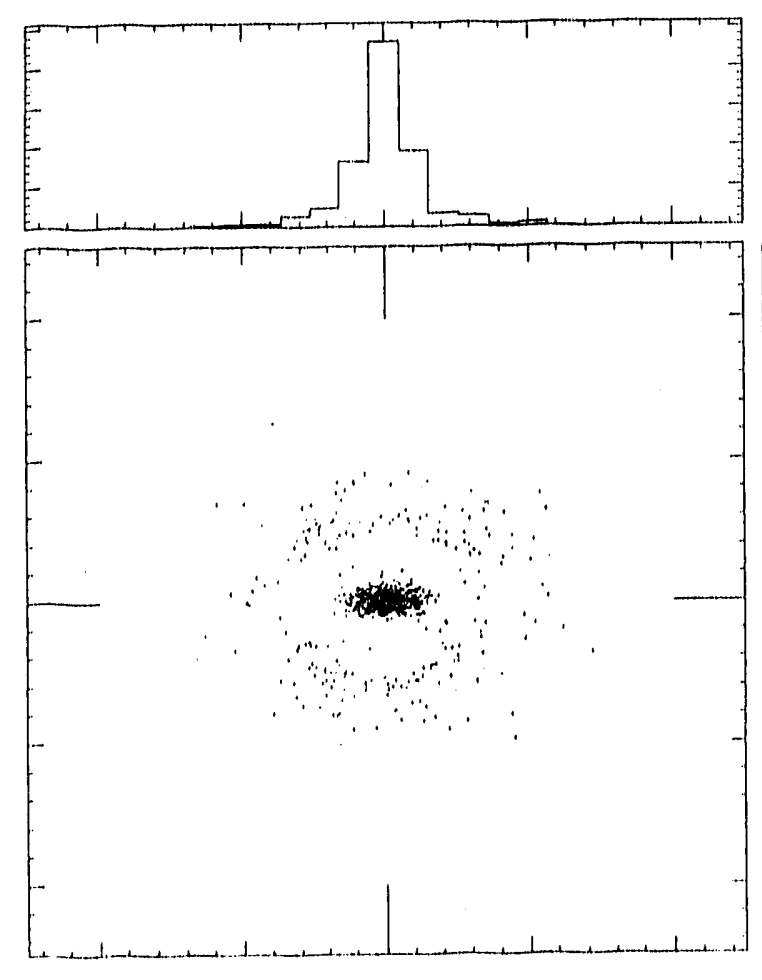

12.APR-1991 08:40

UAS.0 K=1.9F E $=5.03 \mathrm{~K}$

$\theta \mathrm{V}$

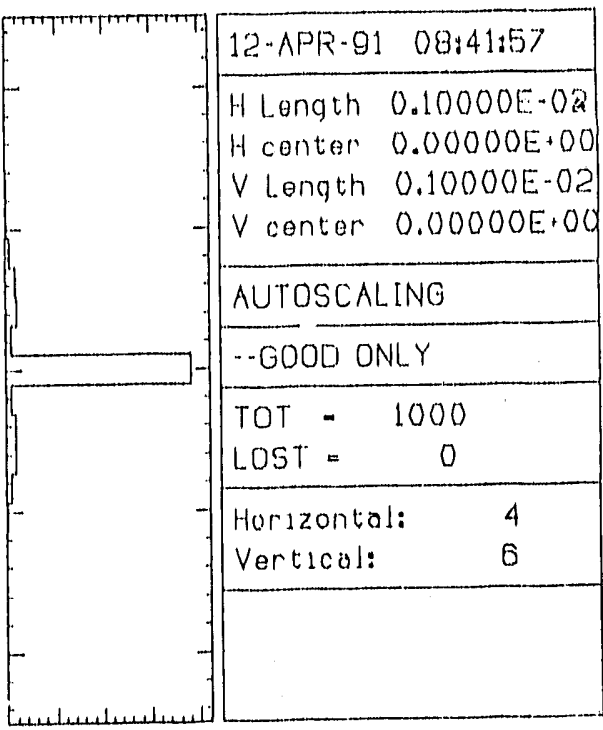

12.APR-199108:45

UAS $-1 K=1.9 F \quad E=5.03 K$

$\theta \mathrm{V}$

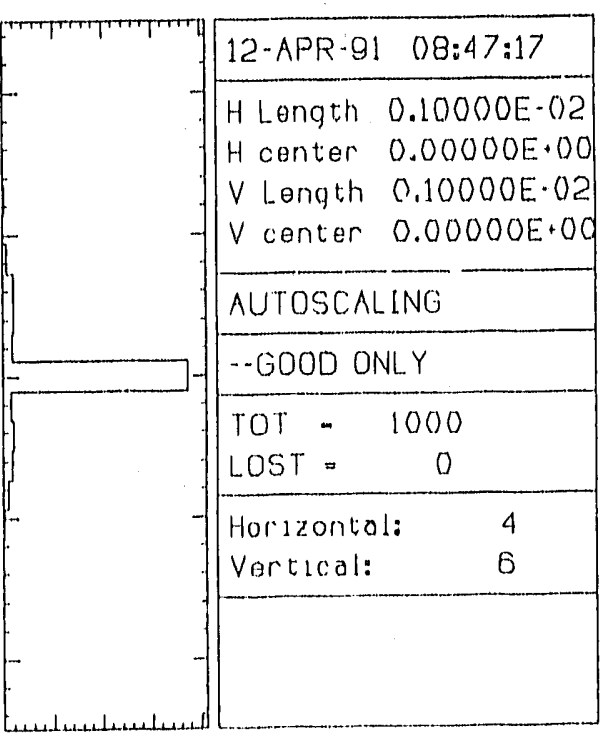

Fig. UA-7. $\quad \mathrm{K}=1.9$, first harmonic. 
USERBDISK:IXUSIBEGIN.OAT:A
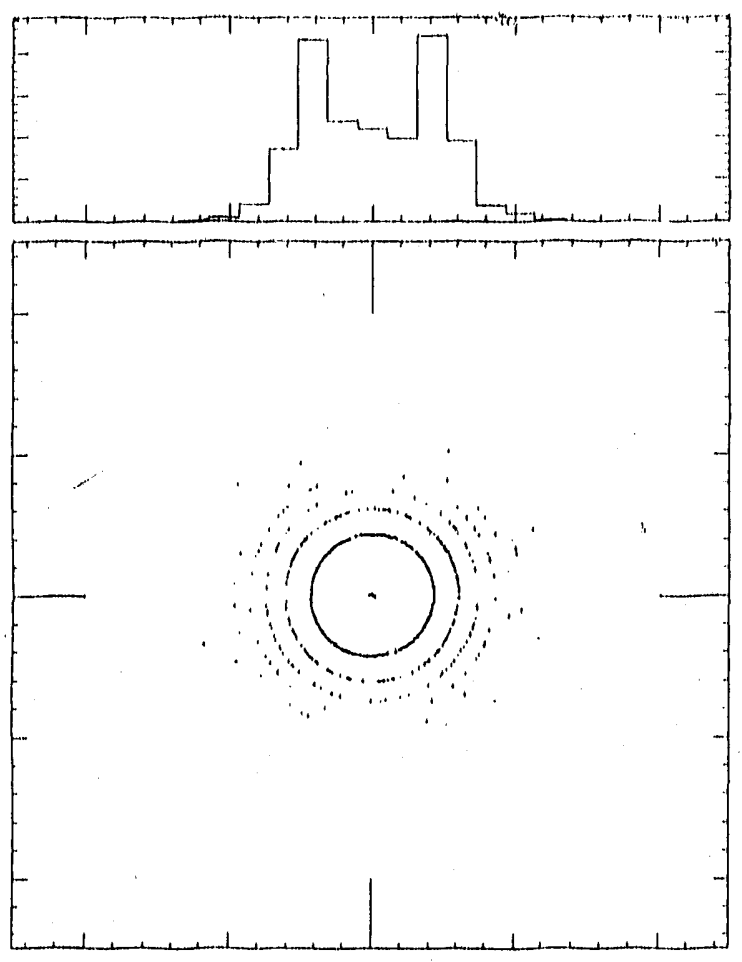

USERBDISK:IXUSIBEGIN.DAT;5
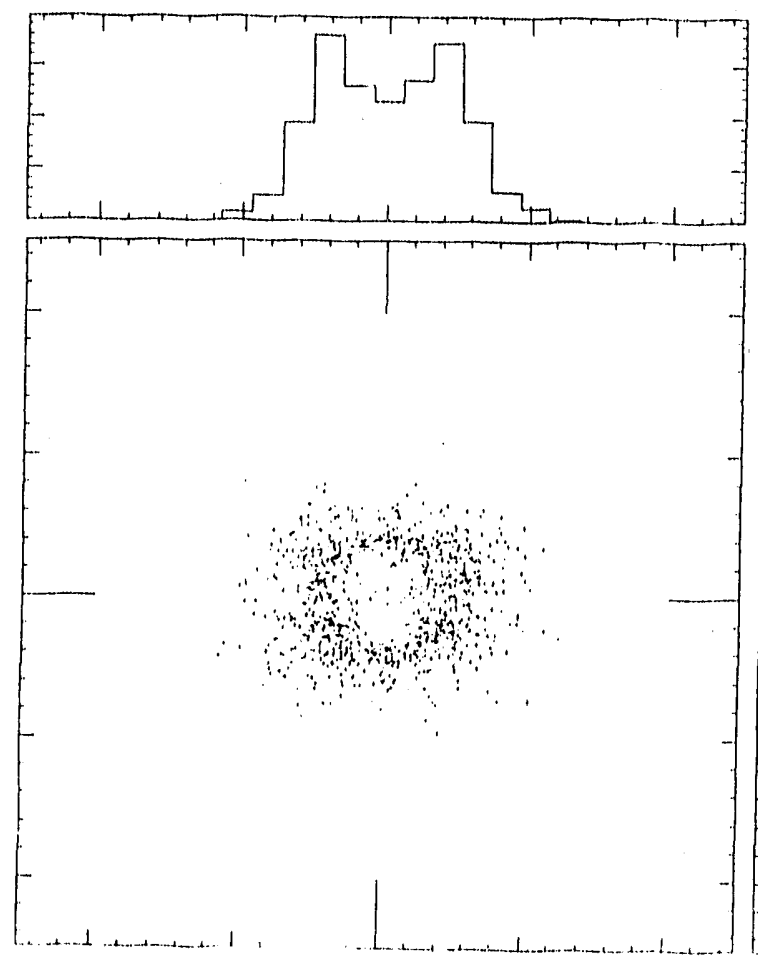

12!-APR-1091108:56

$(14 ; 0) k=1.09 \quad 0: 10.00$

$k 0 V$

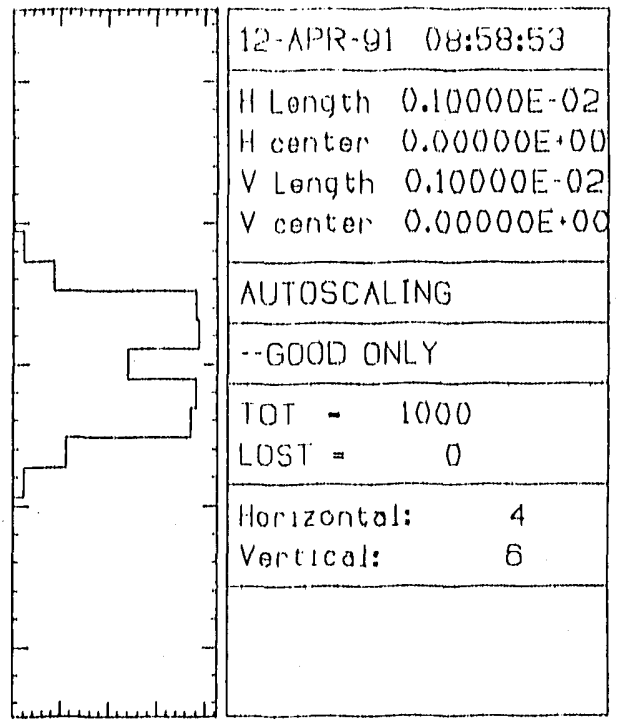

12-APR-199109:01

UAS-1 K=1.9: $E=10.06$

koV

Fig. UA-8. $\mathrm{K}=1.9$, second harmonic. 
USERBDISK:IXUSIBEGIN,DAT;6
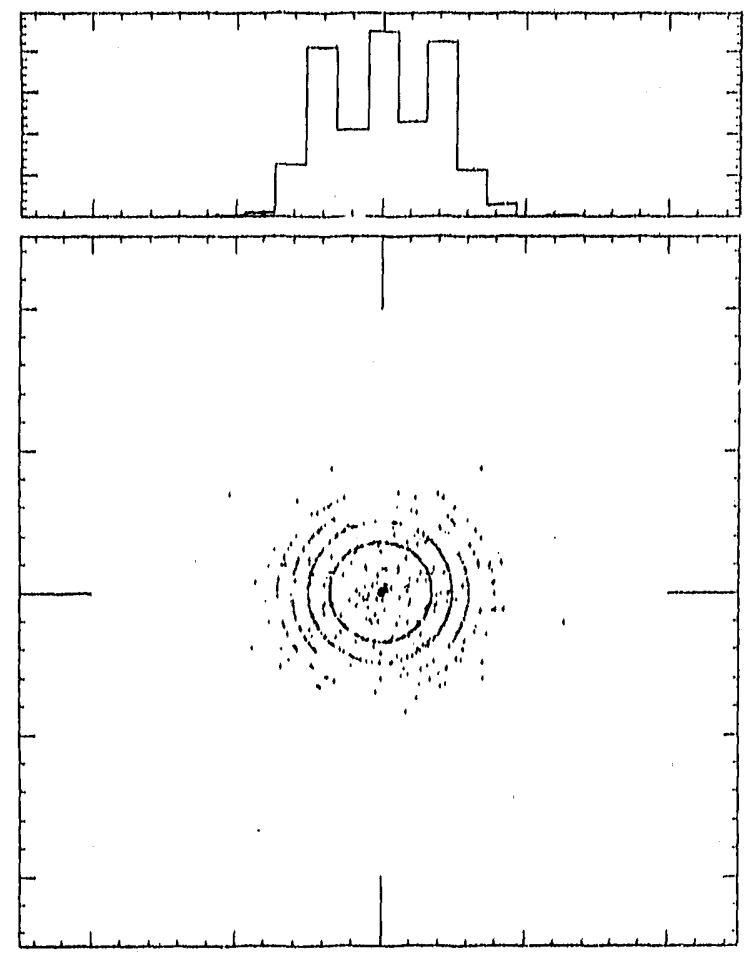

USER DISK:[XUSIBEGIN.DAT:7
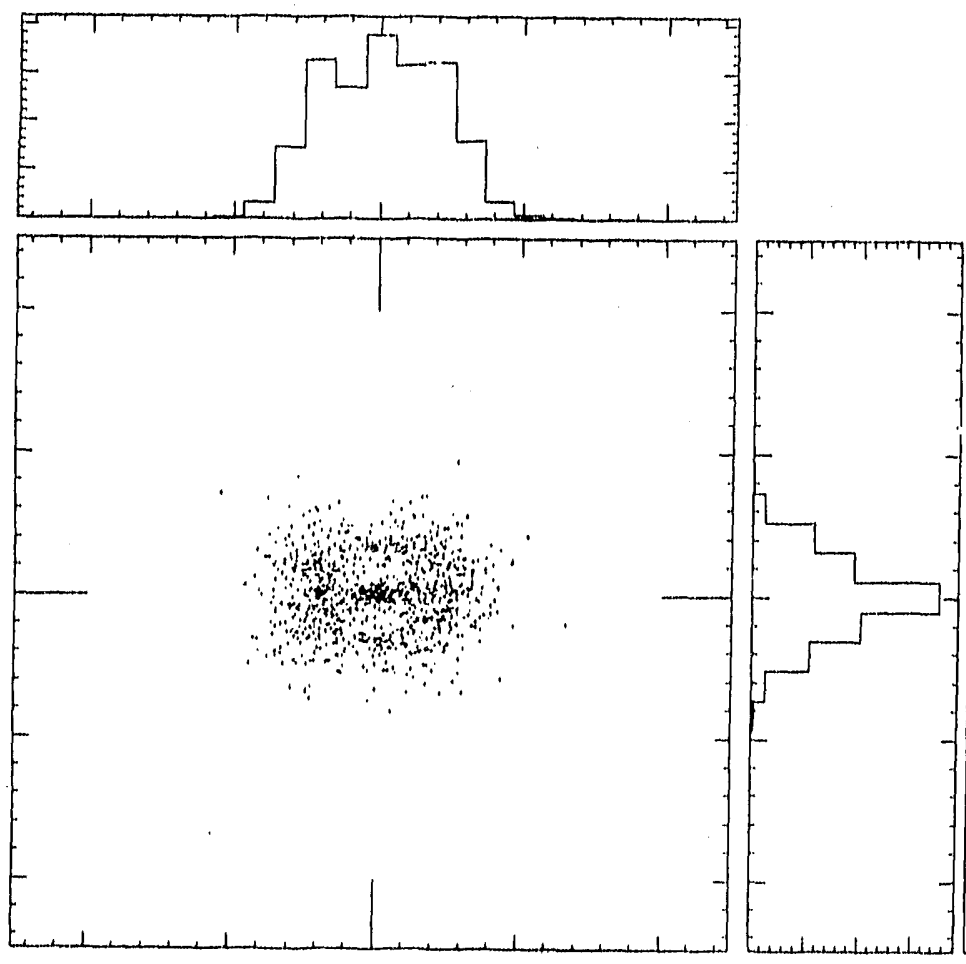

12-APR-1991 09:12

UAS-0 $K=1.9 \mathrm{t} \quad E=15.08$

$k \in V$

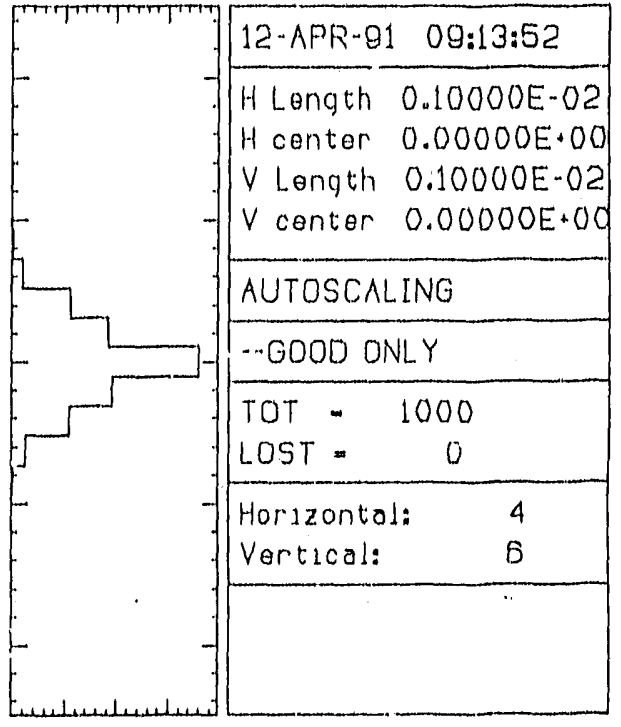

Fig. UA-9. $\quad \mathrm{K}=1.9$, third harmonic. 
USER BISK:[XUS.SHRDOW]BEGIN.DAT;3
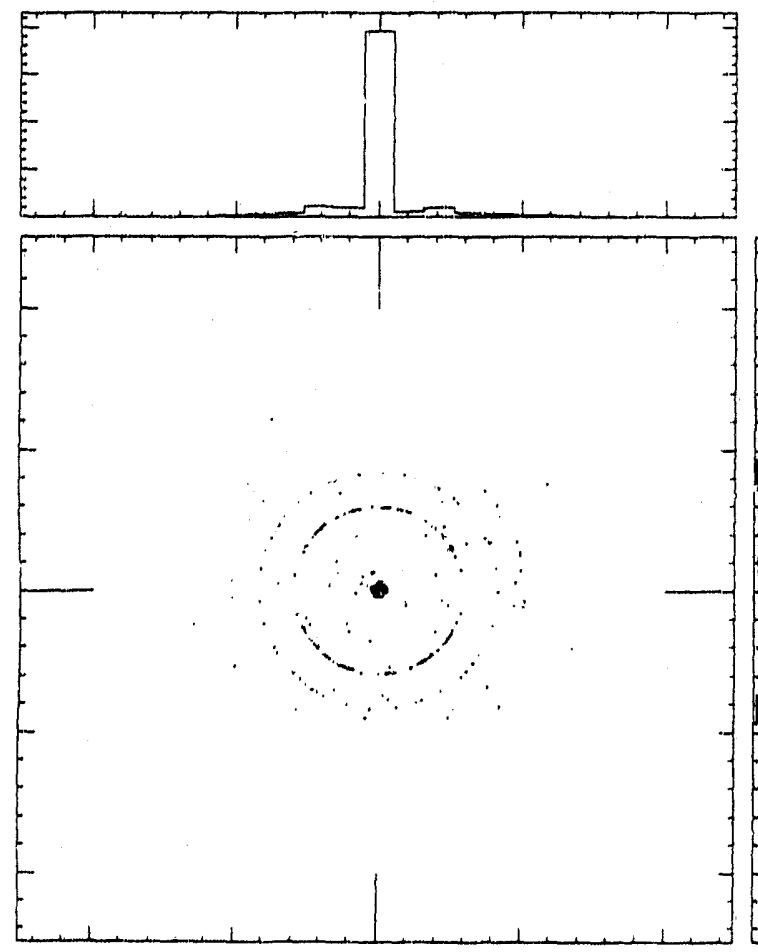

USER PISK:[XUS.SHADOWIBEGIN.DAT;4
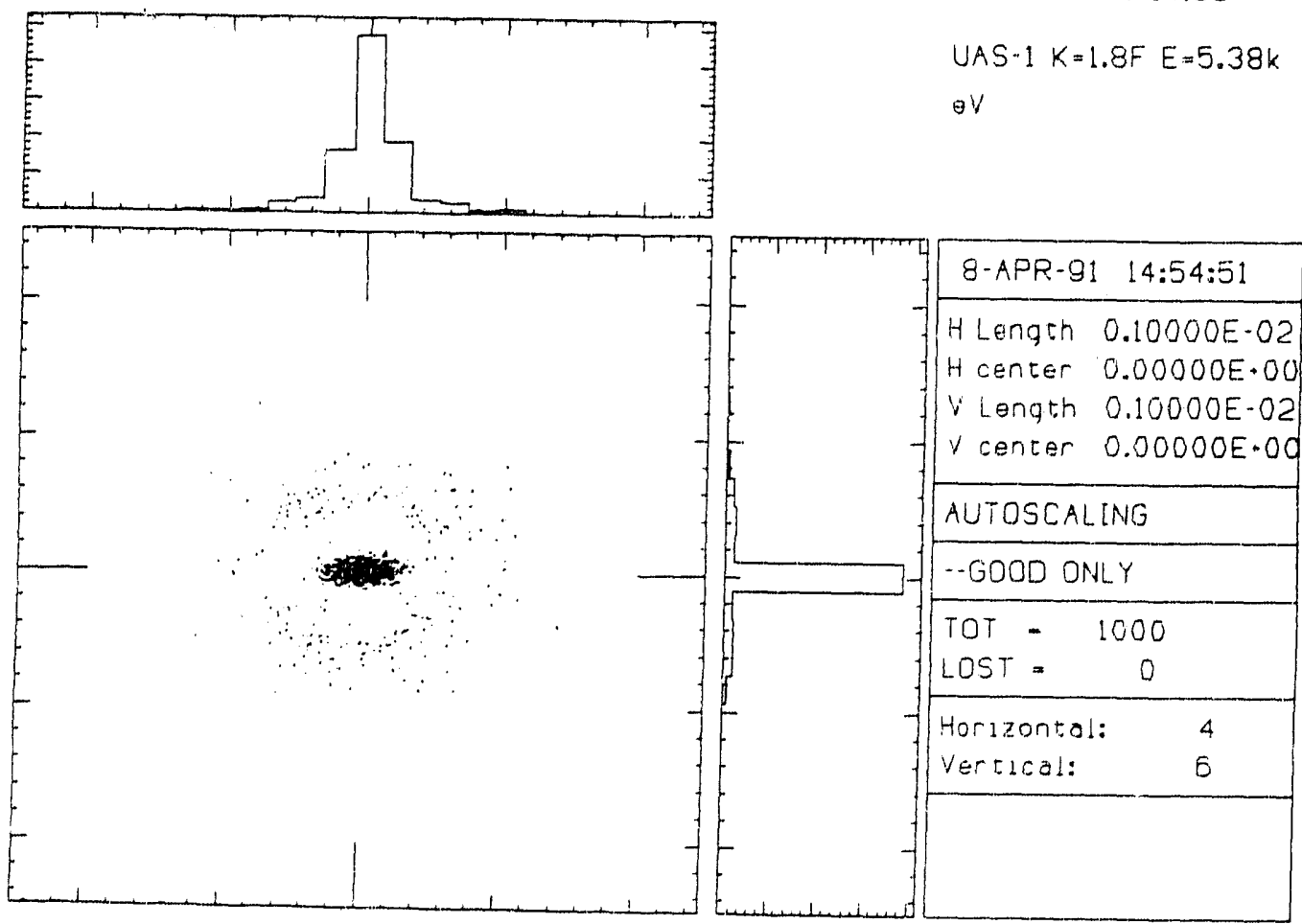

8-APR-1991 $14: 48$

UAS-O $K=1.8 F \quad E=5.38 \mathrm{~K}$

$\theta \mathrm{V}$

\begin{tabular}{|c|c|c|}
\hline \multirow{4}{*}{ 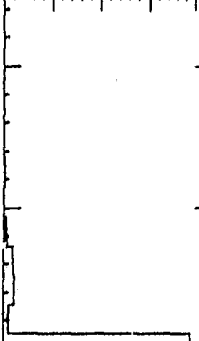 } & \multicolumn{2}{|c|}{ 8-APR-91 $14: 49: 48$} \\
\hline & \multicolumn{2}{|c|}{$\begin{array}{ll}H \text { Length } & 0.10000 E-02 \\
H \text { center } & 0.00000 E-00 \\
V \text { Length } & 0.10000 E-02 \\
V \text { center } & 0.00000 E+00\end{array}$} \\
\hline & \multicolumn{2}{|c|}{ AUTOSCALING } \\
\hline & \multicolumn{2}{|c|}{ - GOOD ONLY } \\
\hline & \multicolumn{2}{|c|}{$\begin{array}{lc}\text { TOT }= & 1000 \\
\text { LOST }= & 0\end{array}$} \\
\hline & $\begin{array}{l}\text { Horizontal: } \\
\text { Vertical: }\end{array}$ & $\begin{array}{l}4 \\
6\end{array}$ \\
\hline & & \\
\hline
\end{tabular}

8-APR-1991 14:53

UAS $1 K=1.8 F \quad E=5.38 K$

QV

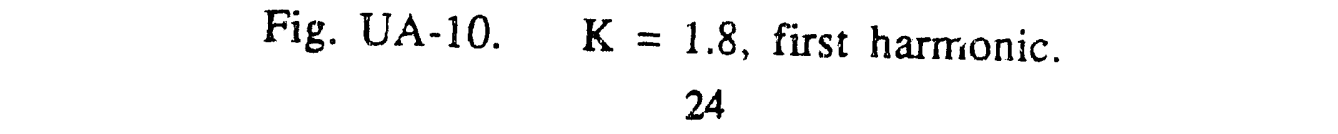




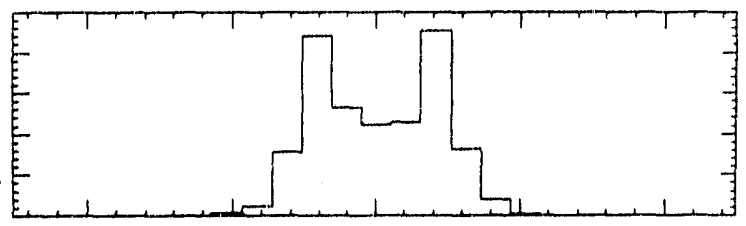

UAS-C $K=1.8 \mathrm{~s} E=10.76$

$k \in V$

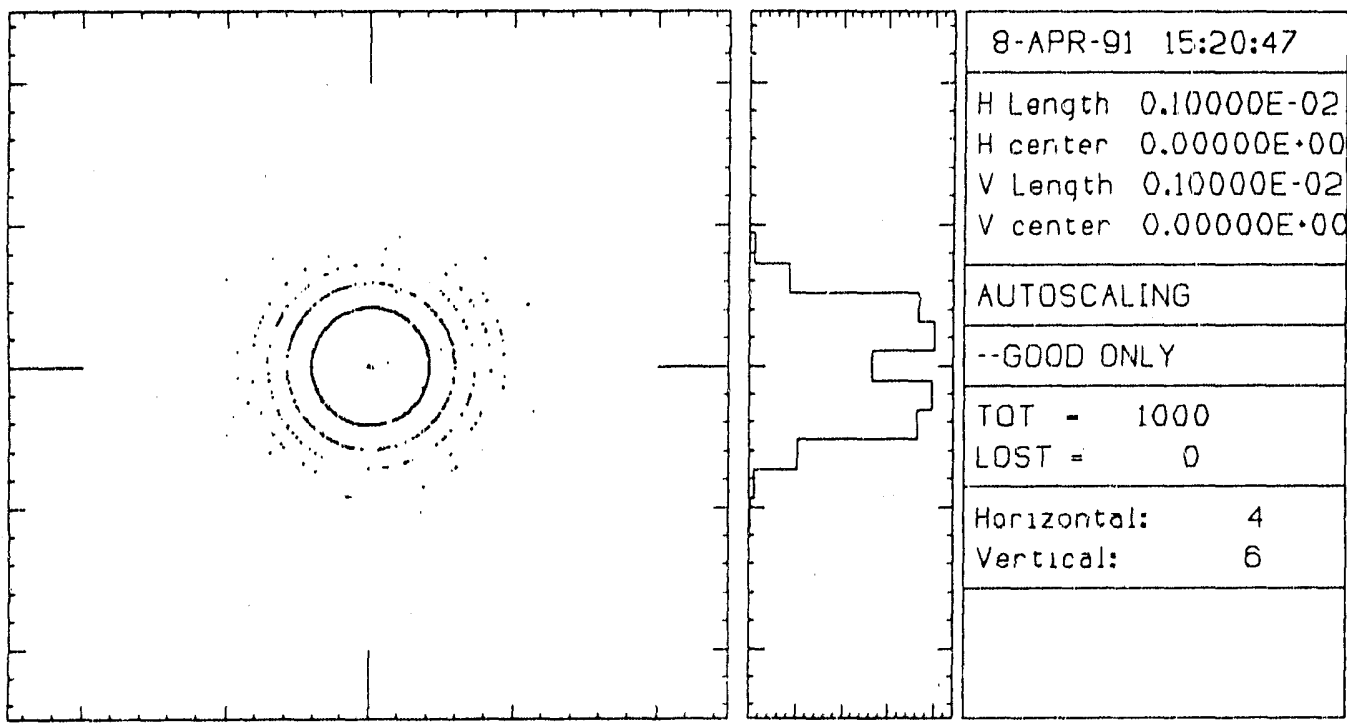

USER DISK:[XUS.SHADOW]BEGIN.DAT;O
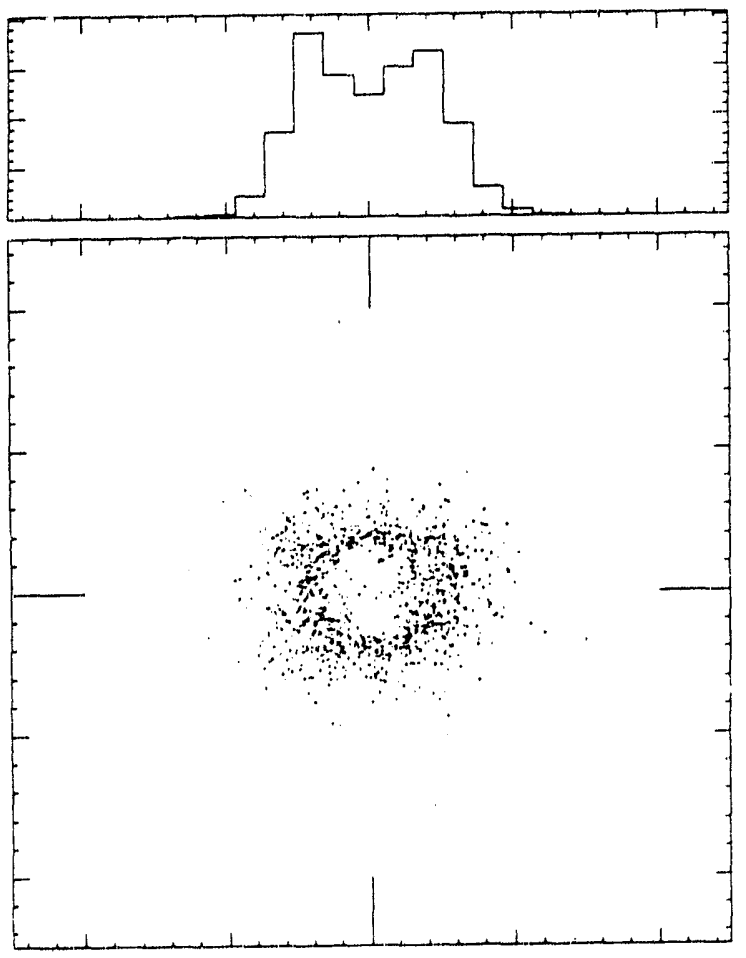

Q-APR-1991 15:23

UAS-1 $K=1.8 \mathrm{~s} \quad E=10.76$

$k \theta V$

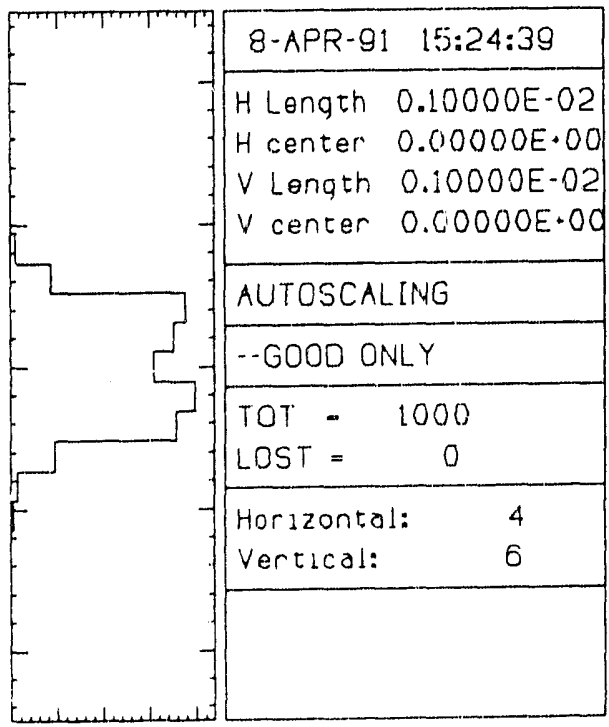

Fig. UA-11. $\quad \overline{\mathrm{K}}=1 . \overline{8}$, second hăminonic. 
USER 1OISK:[XUS.SHADOW]BEGIN.DAT;7
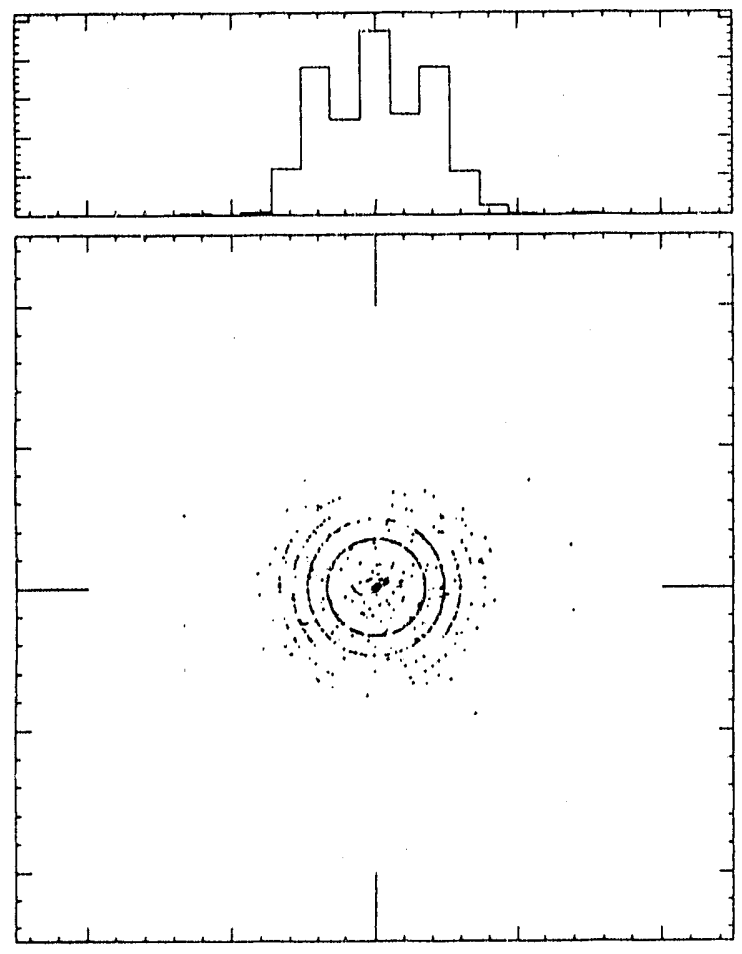

USER \&ISK:[XUS.SHADOW]BEGIN.DAT;8
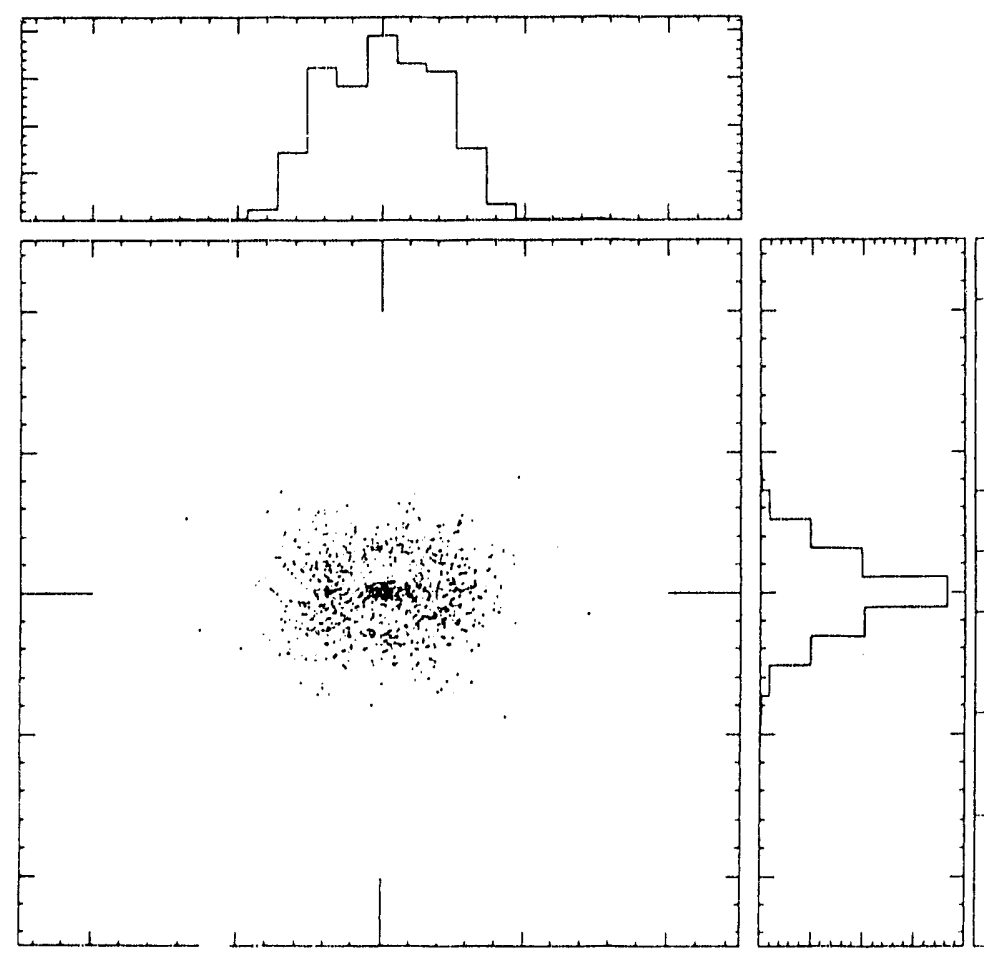

8-APR-1991 15:33

UAS-O $K=1.8 T E=16.15$

$k \in V$

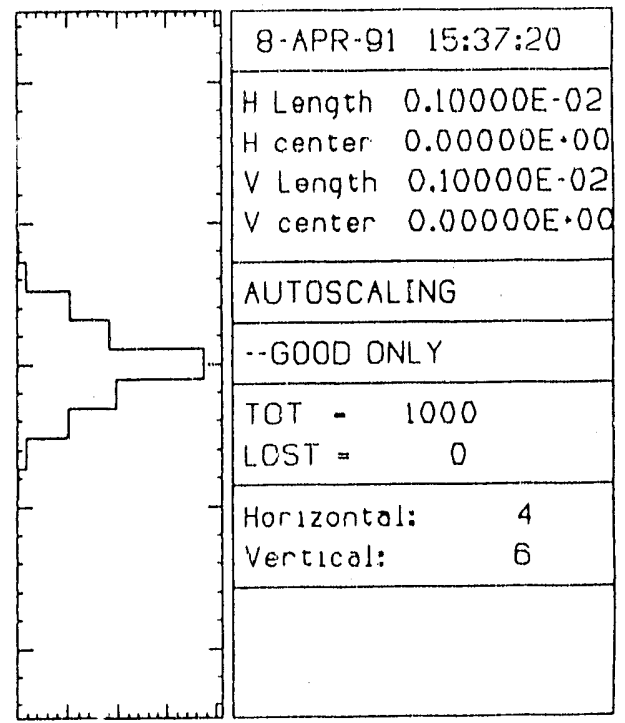

8-APR-1991 15:41

UAS - $1 \quad K=1.8 T E=16.15$

keV

8-APR-91 15:43:04

$H$ Length $0.10000 E-02$

$H$ center $0.00000 E \cdot 00$

$V$ Lenqth $0.10000 E-02$

$\checkmark$ center $0.00000 E \cdot 00$

AUTOSCALING

-GDOD ONLY

TOT - 1000

LOST $=0$

Horizontol. 4

Vertical: 6

Fig. UA-12. $\mathrm{K}=1.8$, third harmonic. 
USER DISK:[XUS.SHADOW]BEGIN.DAT:1
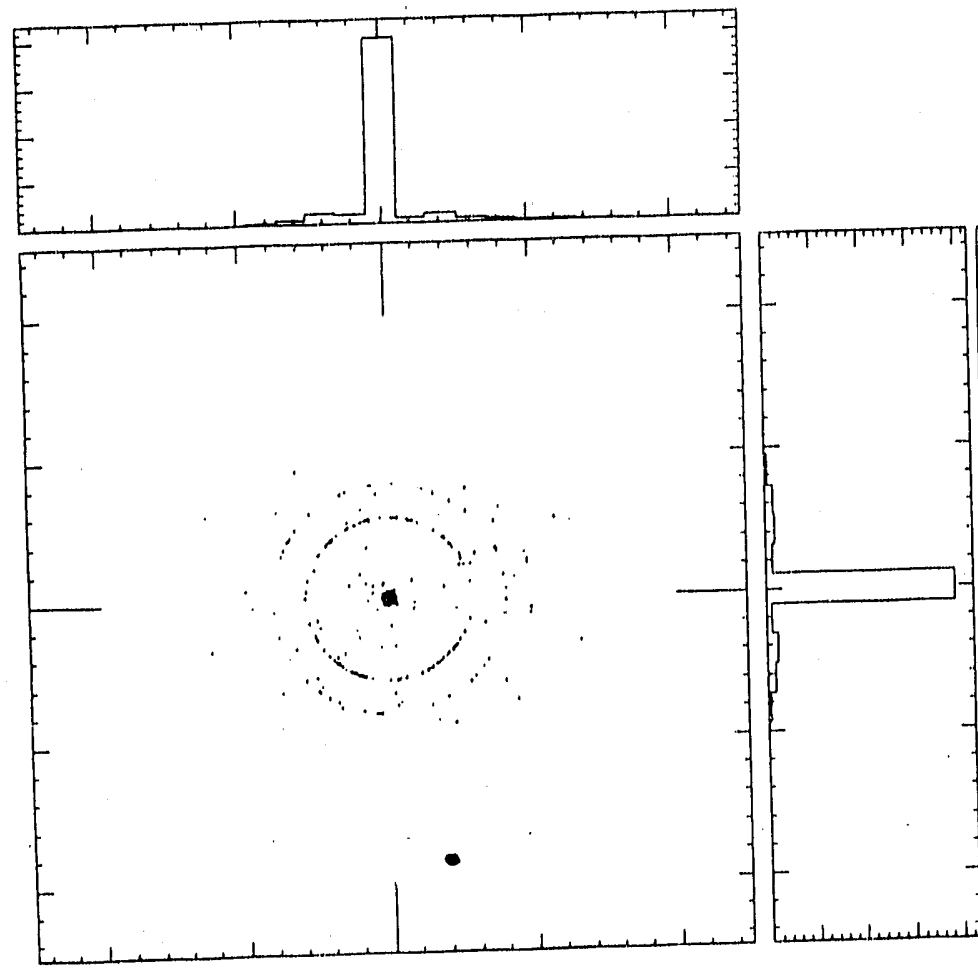

12-APR-1991 14:15

UAS-O $K=1.7 F \quad E=5.77 \mathrm{~K}$

eV

12-APR-91 14:17:07

H Length 0.10000 E-02

$H$ center $0.00000 E+00$

$V$ Lenqth $0.10000 E-02$

$\checkmark$ center $0.00000 E+00$

AUTOSCALING

-.GOOD ONLY

TOT - 1000

LOST $=0$

Horizontal: 4

vertical:

6

12-APR-1991 14:20

USERBDISK: (XUS.SHADOW]BEGIN.DAT;2

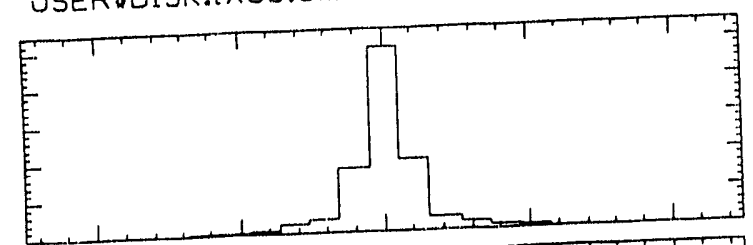

UAS-1 $K=1.7 F \quad E=5.77 K$

$\mathrm{eV}$

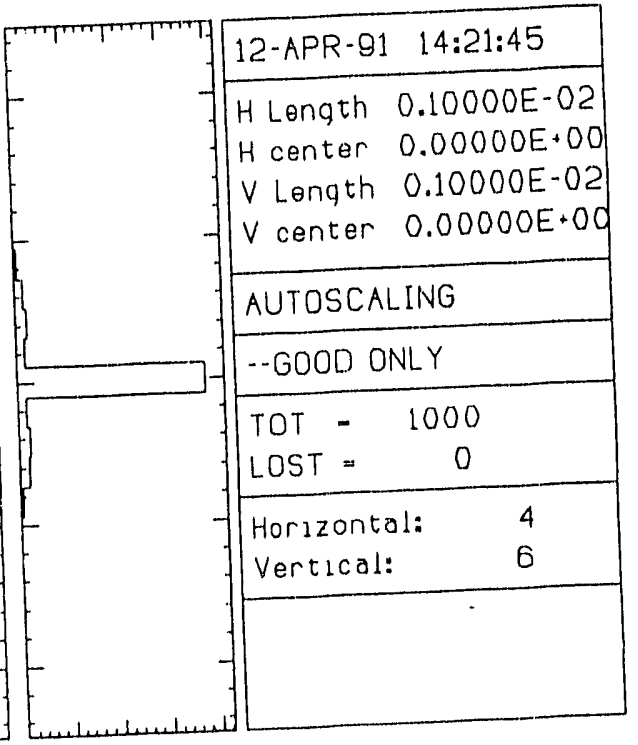

Fig. UA-13. $\quad \mathrm{K}=1.7$, first harmonic. 

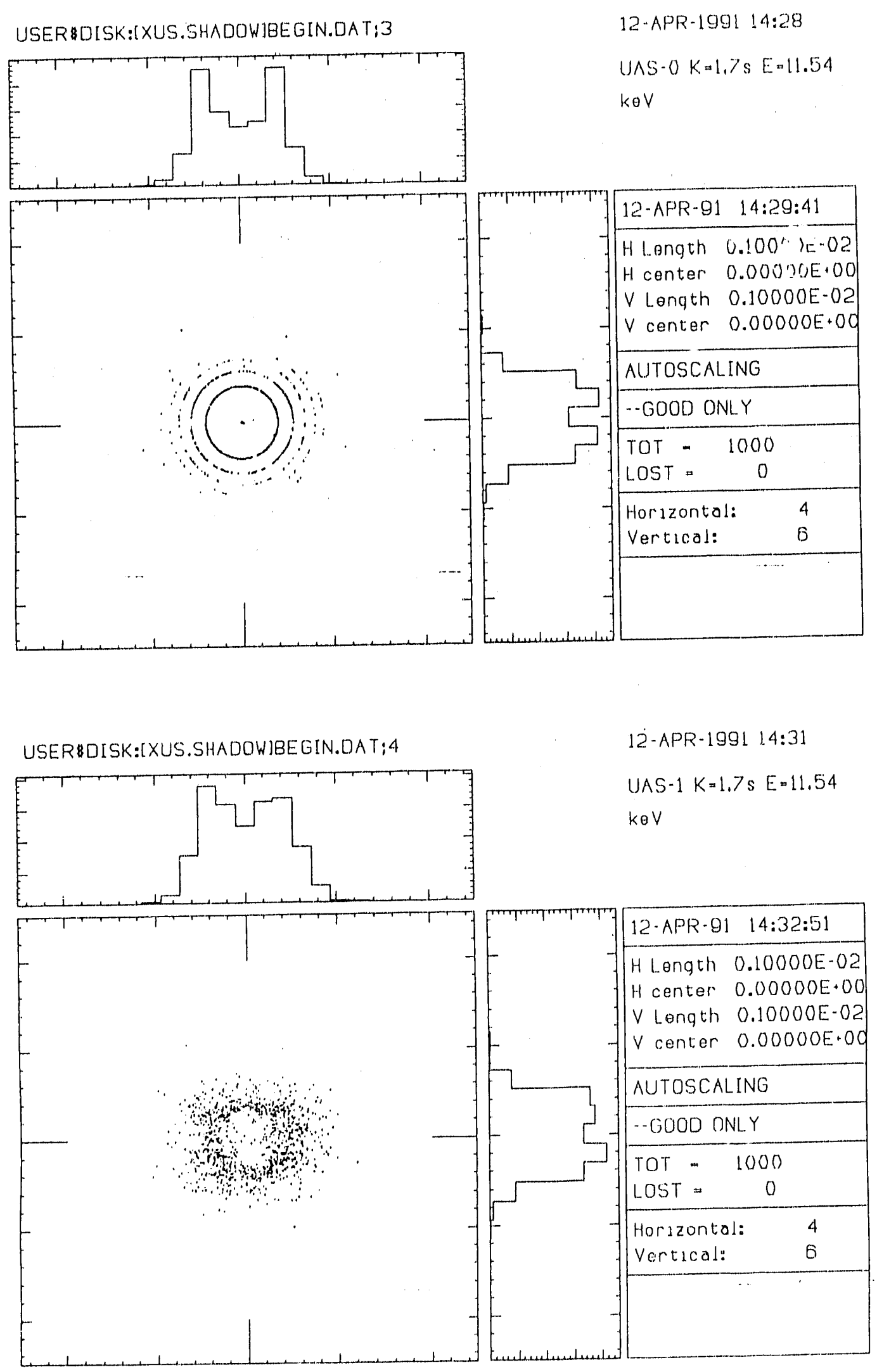

Fig. UA-14. $\quad \mathrm{K}=1.7$, second harmonic. 
USERBDISK:IXUSIBEGIN.MAT;O
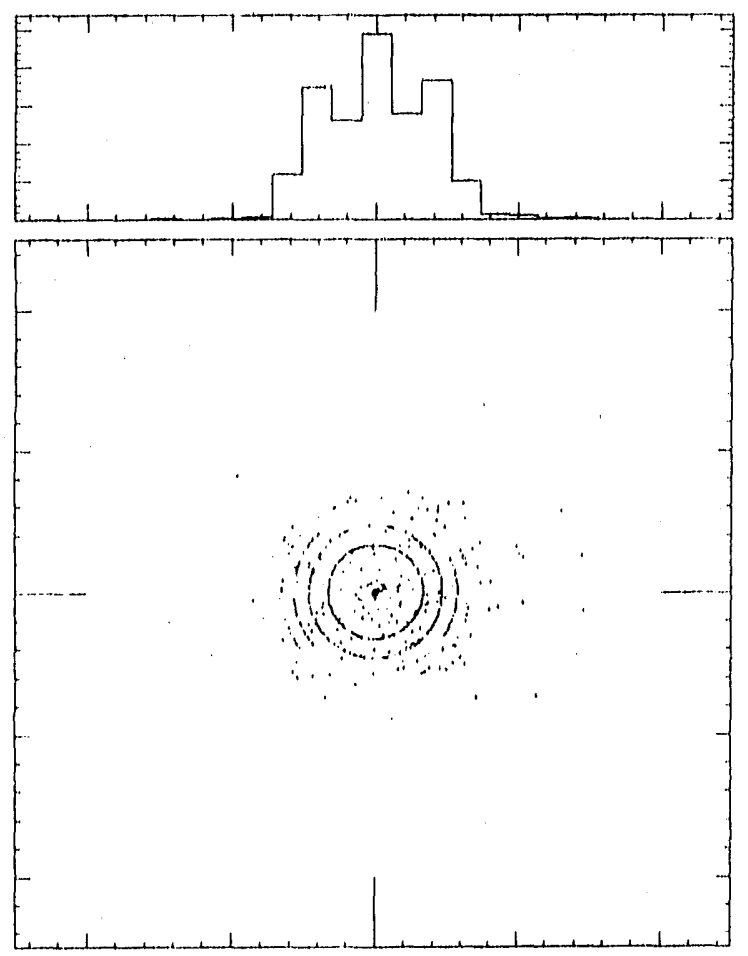

USER \&OISK:IXUS.SHADOW]BEGIN.DAT;5
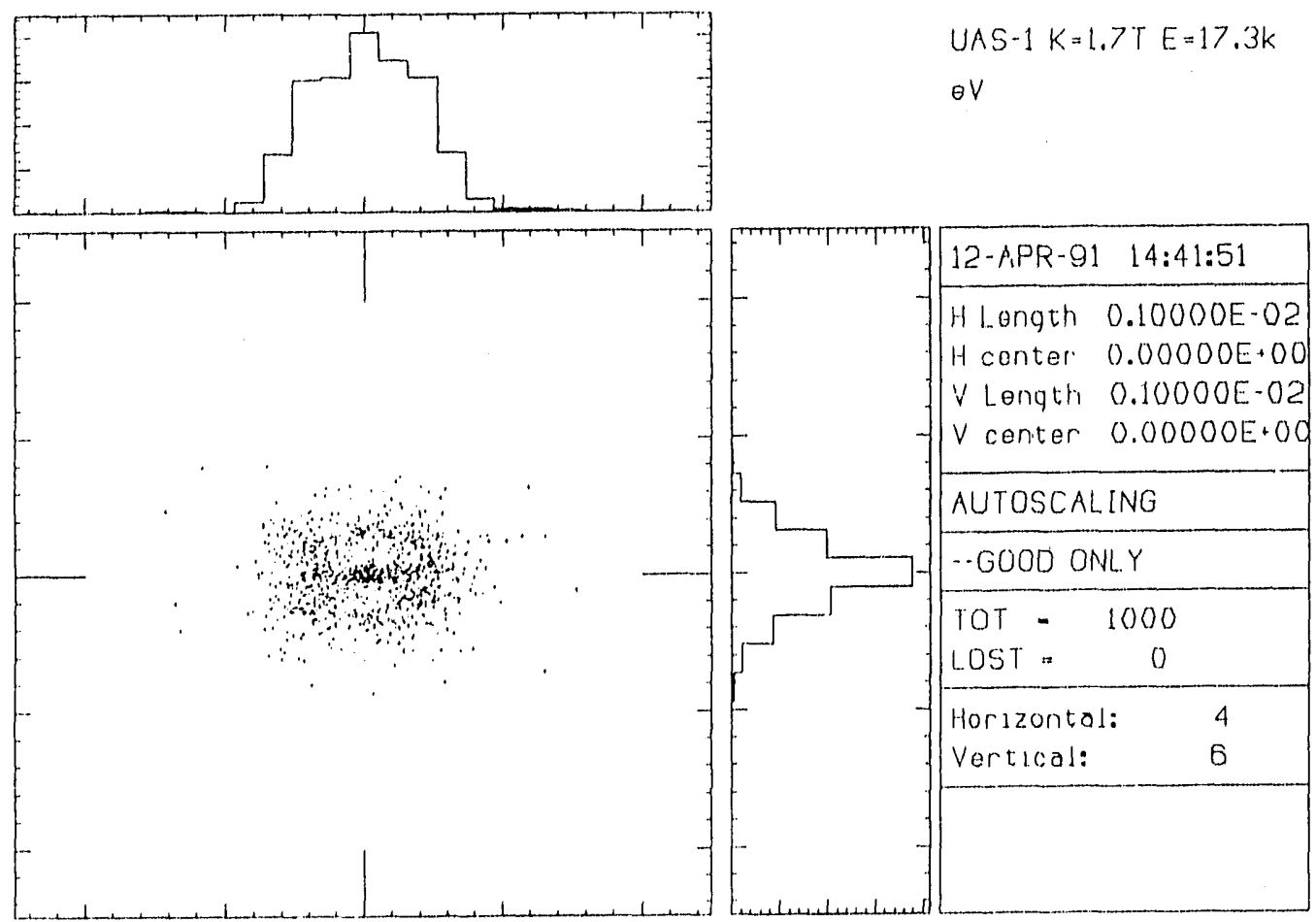

Fig. UA-15. $\quad \mathrm{K}=1.7$, third harmonic. 


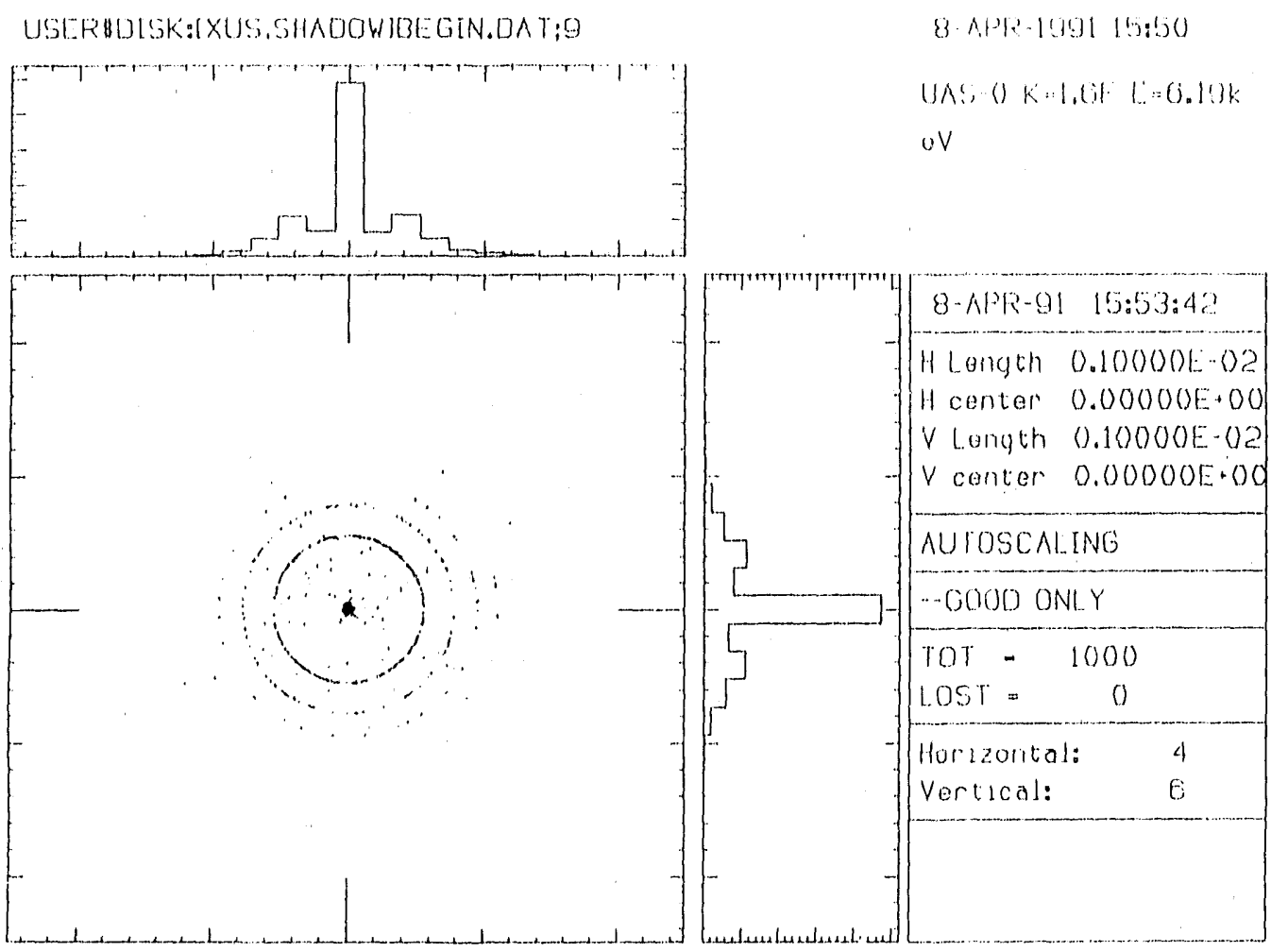

USERHDISK:[XUS.SHADOW]BEEIN.DAT;10

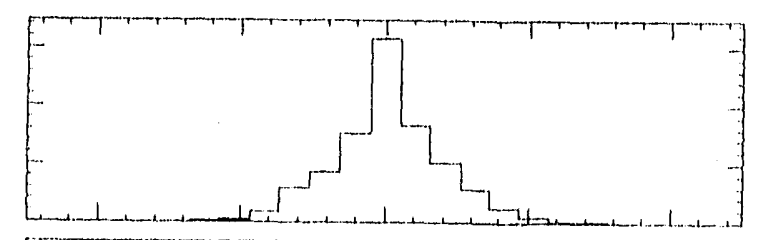

8-APR-1991 15:55

$U A S \cdot 1 K=1.6 \% E=0.19 k$

$\theta \mathrm{V}$

Fig. UA-16. $\mathrm{K}=1.6$, first harmonic. 


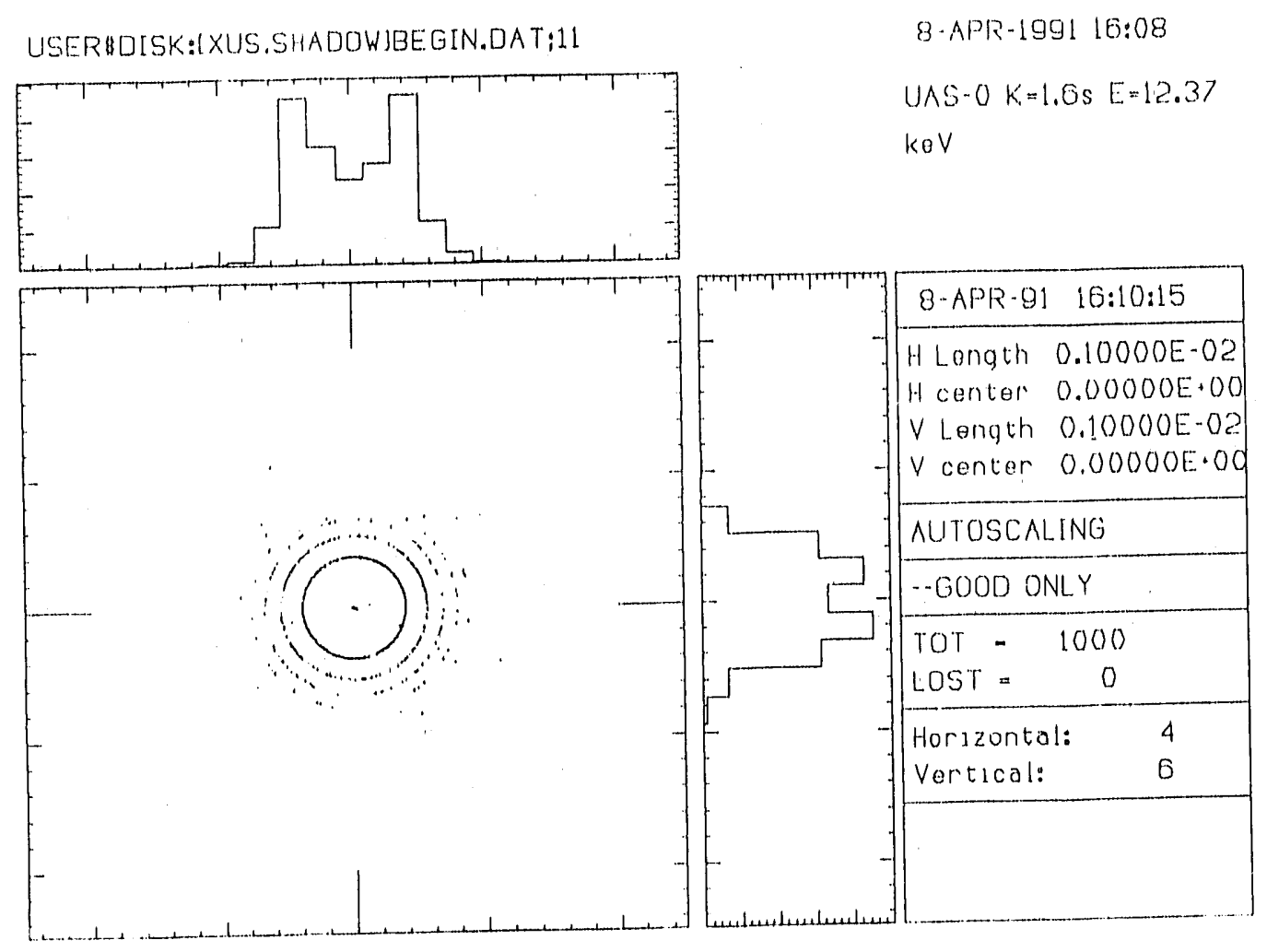

USERBDISK:[XUS.SHADOWJBEGIN.DAT;12
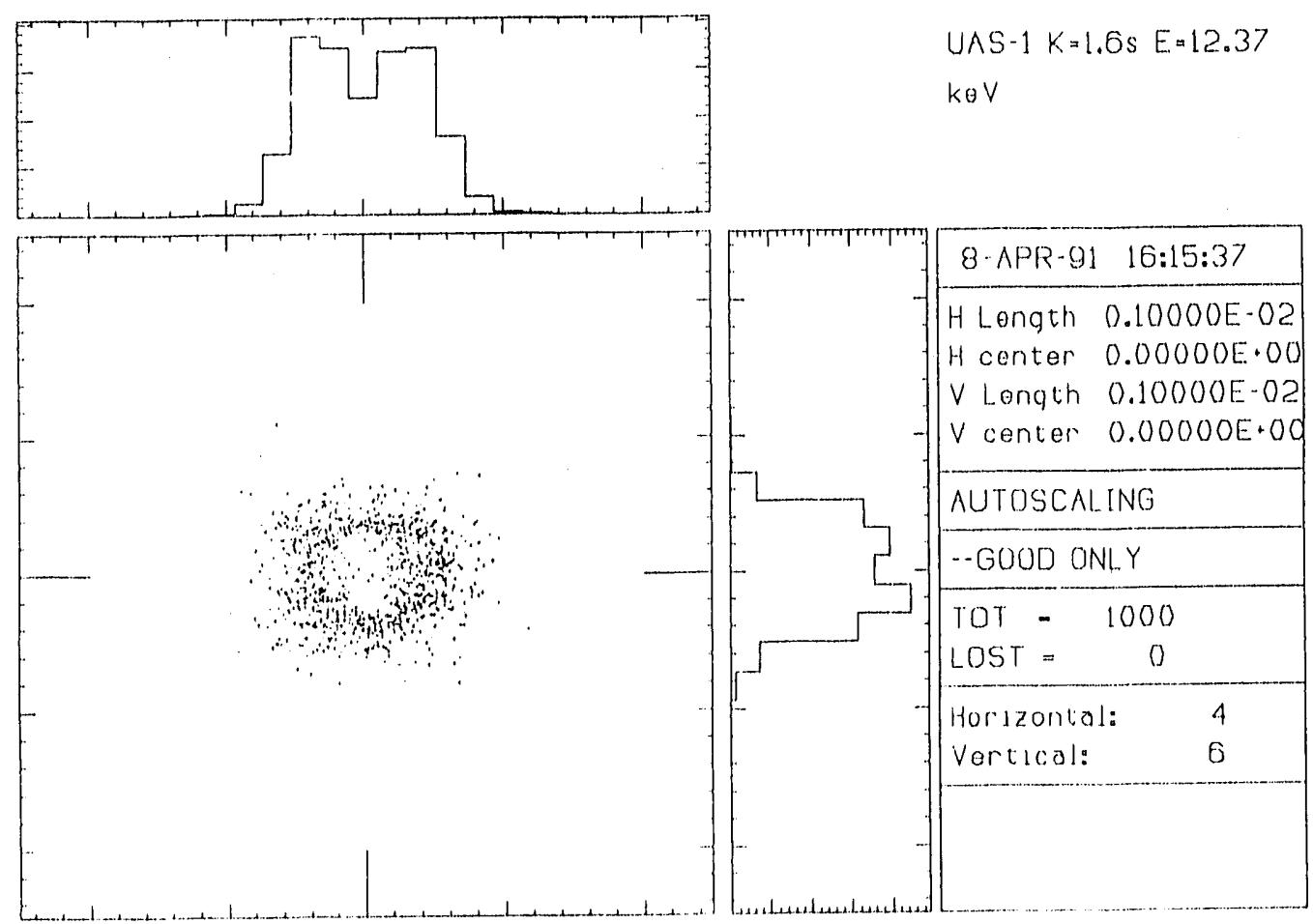

8-APR-1991 16:13

UAS-1 $K=1.6$ S $E=12.37$

$k \in V$

Fig. UA-17. $\mathrm{K}=1.6$, second harmonic. 
USER8DISK:IXIJ:SHADOWIBE GIN.DA T:1
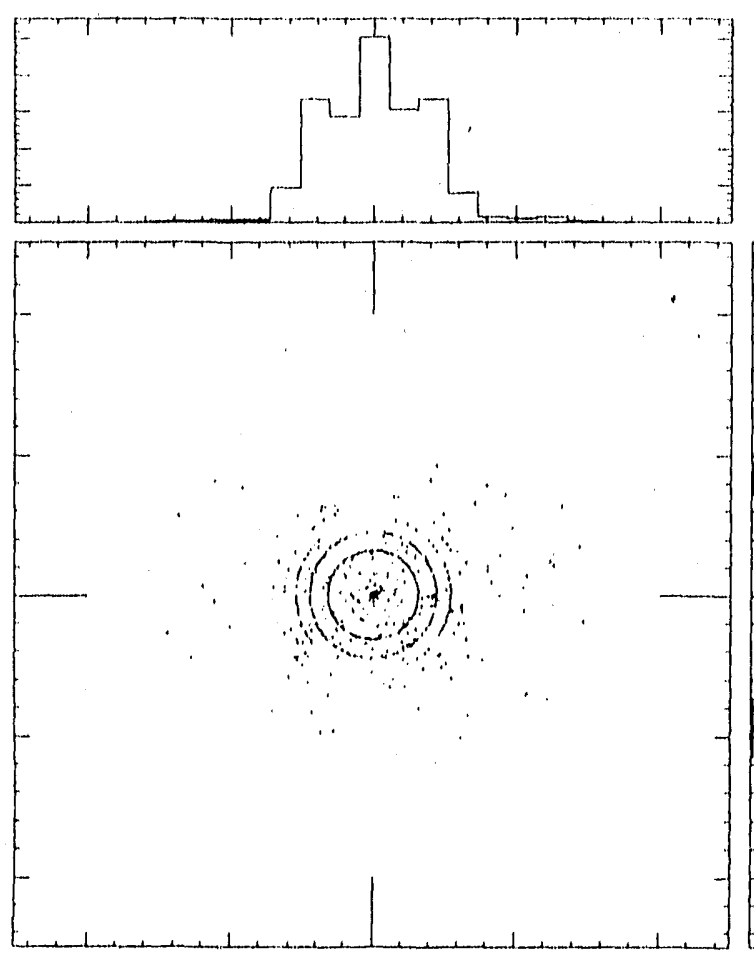

is Alk 1941 110:?

UAs $0 K=1.0111: 183.56$

HeV
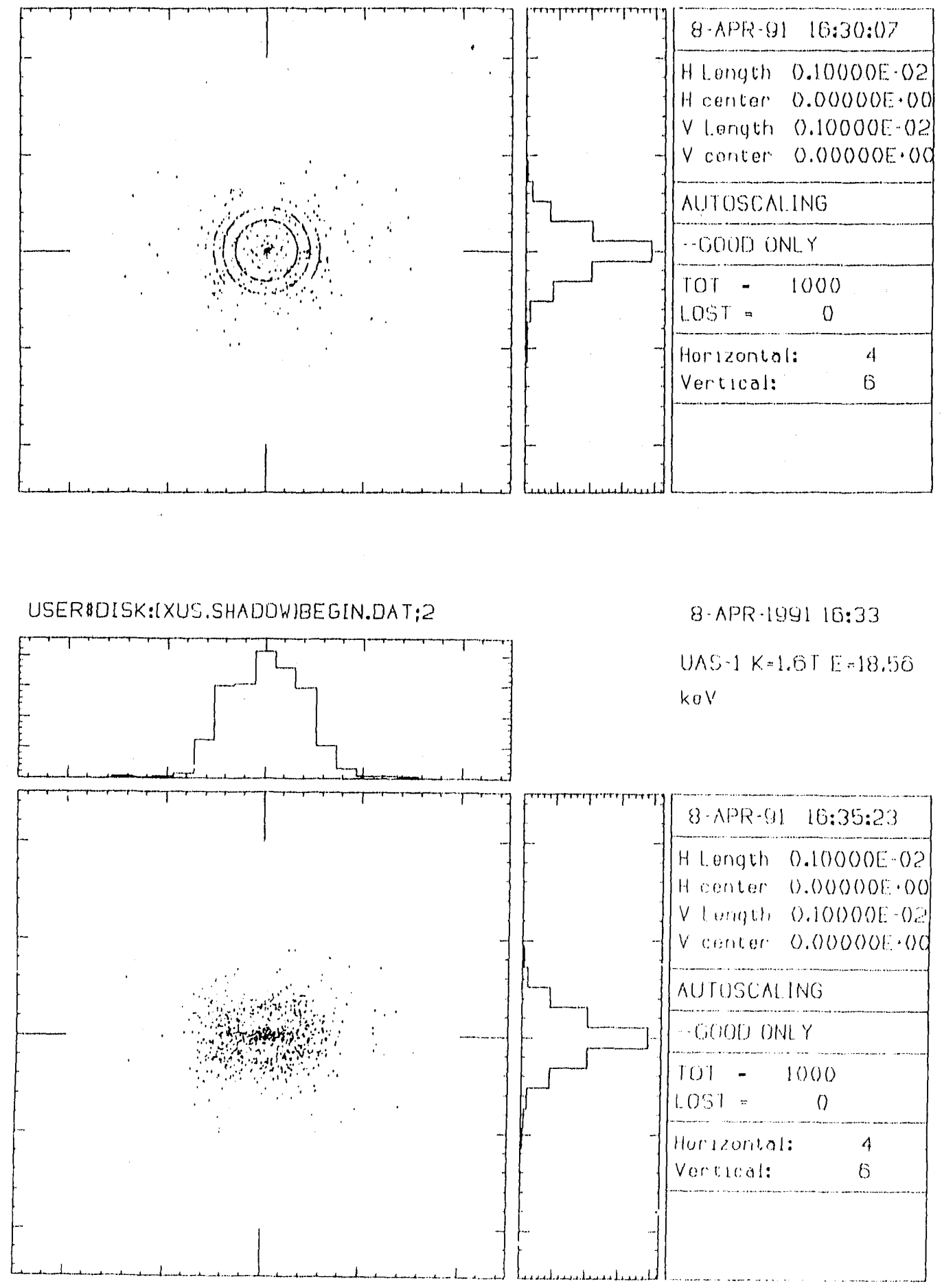

Fis. UA-18. $\quad k=1.0$, third harmonic. 


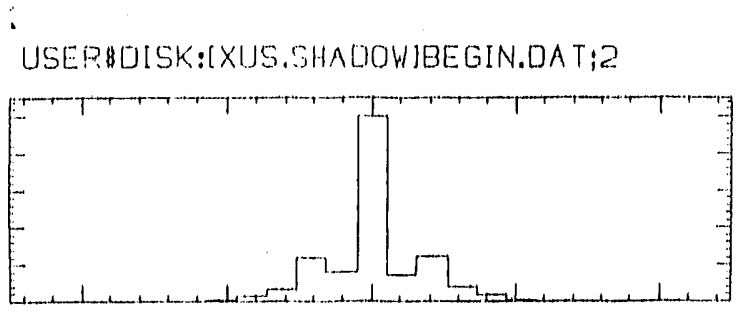

$25 \cdot M A 1 \cdot-1991 \quad 08953$

$U \wedge S-0 k=1.61=6.6 \mathrm{keV}$
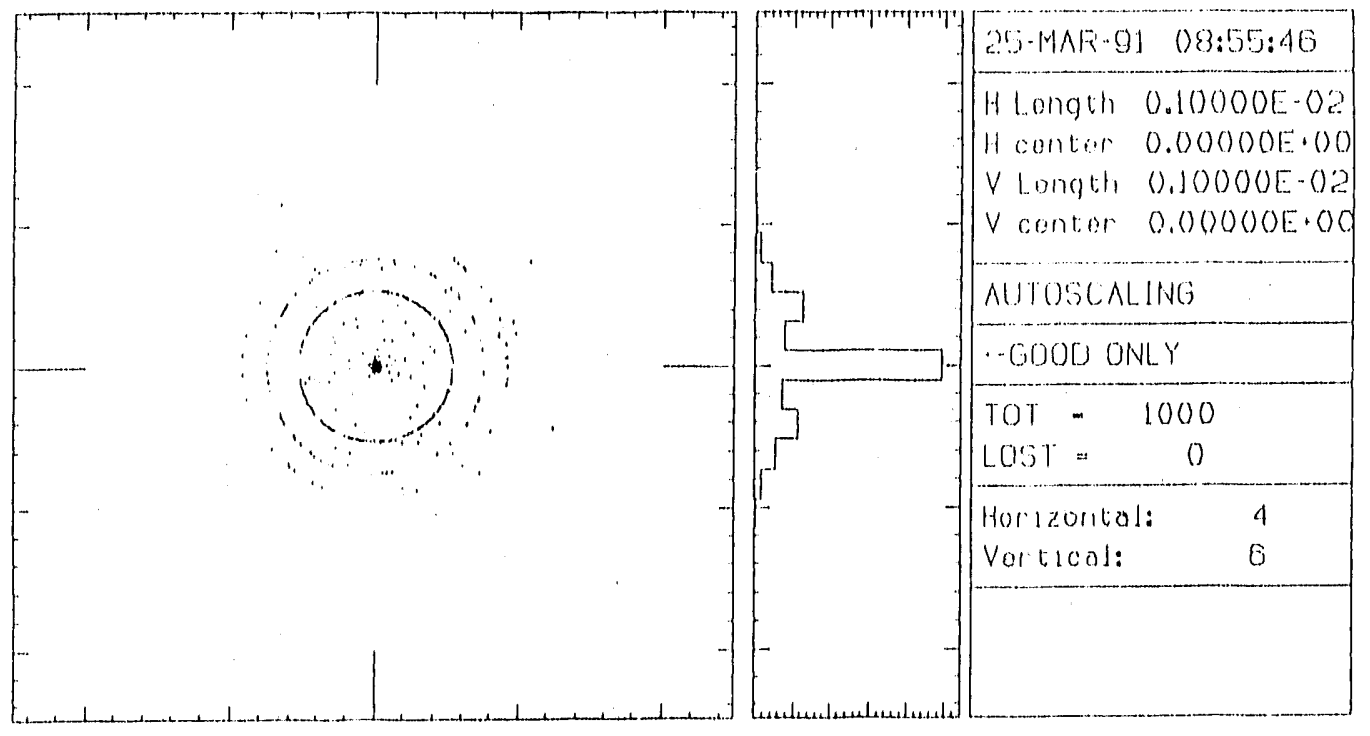

USERBDISK:LUS.SHAOOWIBEGIN.DAT;1
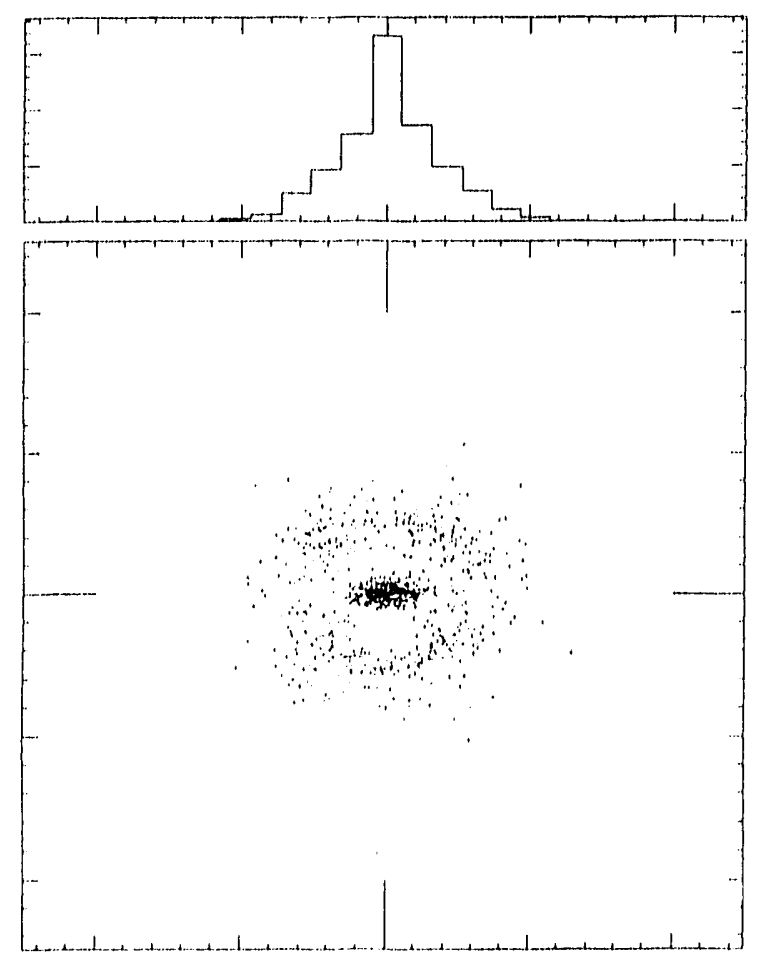

25-MAR-1991 08:4\%

UAS-1 K. $=1.5 E=0.0 \mathrm{k} \cdot \mathrm{V}$

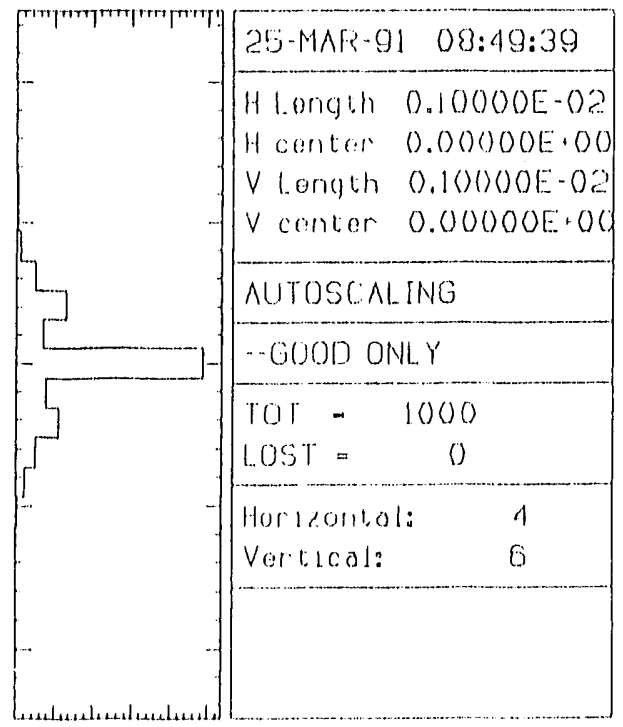

Fig. UA-19. $\quad \mathrm{K}=1.5$, first harmonic. 
I.ISERRDISK:IXUS.SHAOOWIBEOEIN.OAT:3
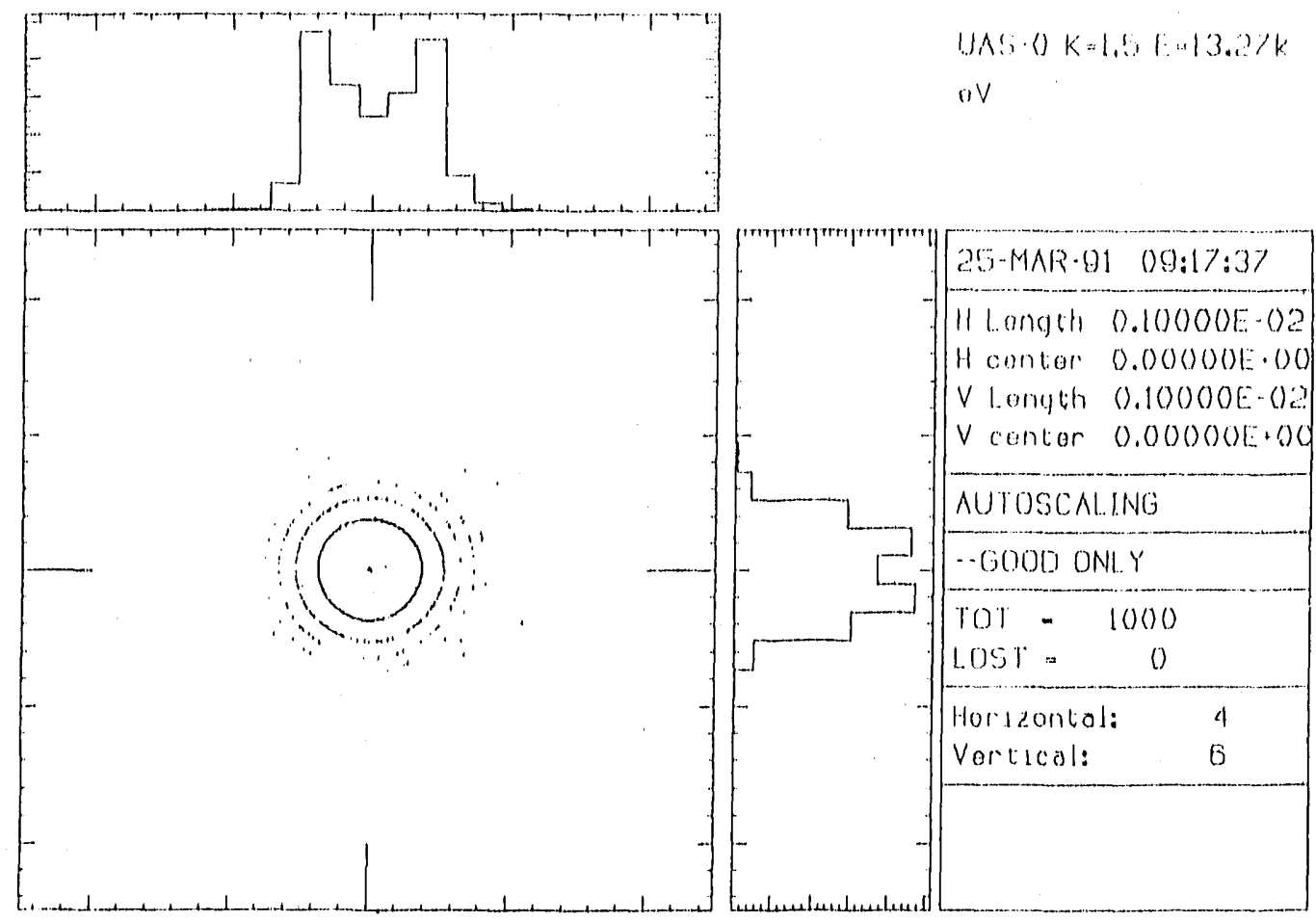

USERBDISK:IXUS.SHADOWIBEGIN.DAT;4
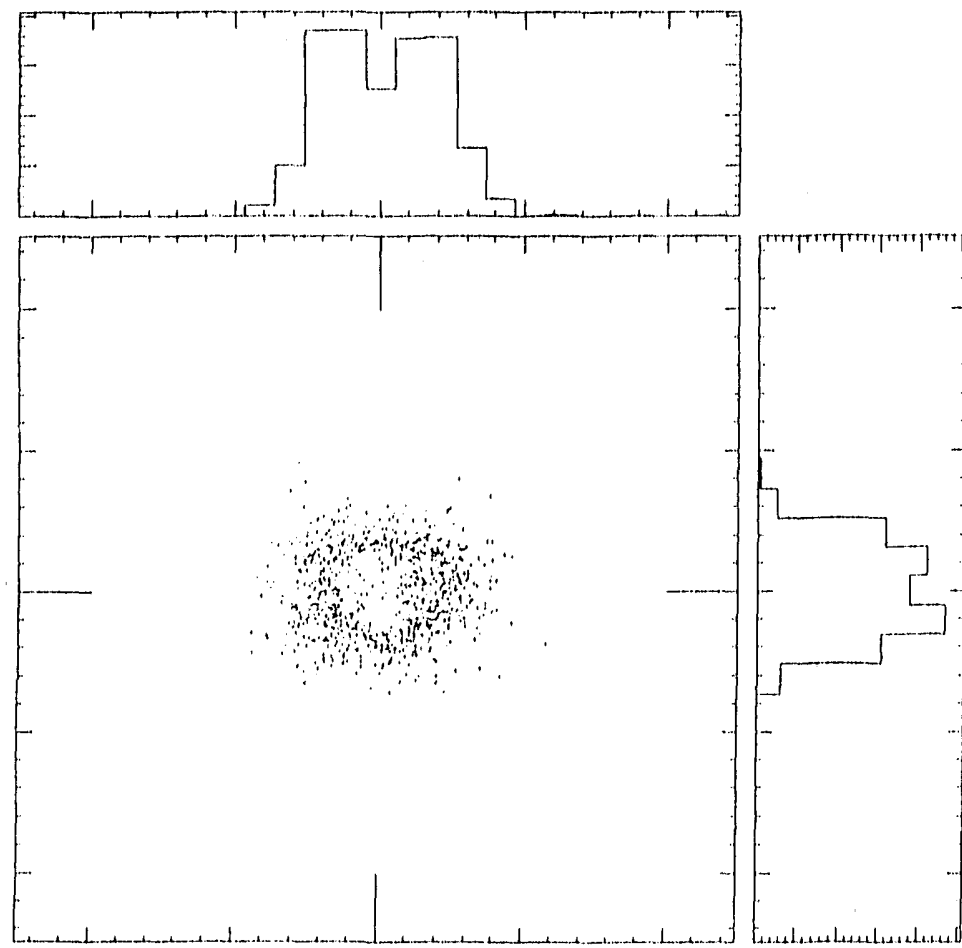

2.) $+14 R \cdot 199189116$

$1 . M S \cdot 3) k=1,61:=13.2 \% k$

$o \mathrm{~V}$
20 MAR-01 $09: 17: 37$

4 conter 0.000000 .00

$\checkmark$ longth $0.10000 E-(1)$ ?

AUTOSCALING

$-Q 000$ ONI. Y

TOT - 1000

Los $=0$

Hortzontal:

Vortical: $\quad$ b

$25-1 \cdot 1 A R-1901099: 21$

UAS $K=1.9 k=13.9 \% k$

oV
$V$ centior $0.00000(0)+00$

Fig. UA-20. $\mathrm{K}=1.5$, second harmonic. 
USERHOISK: XUS.SHAOUWIBEGIN.DATI7
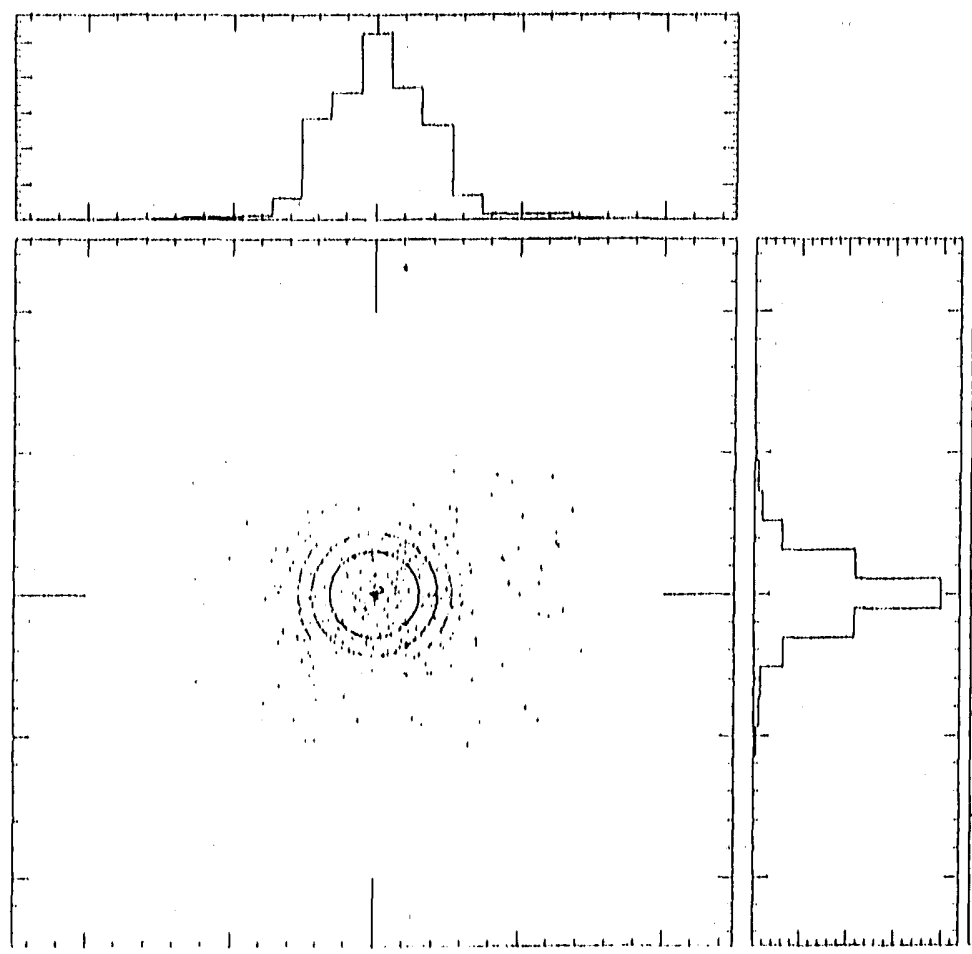

USERBDISK:IXUS.SHADOWIBEGIN.DAT:B
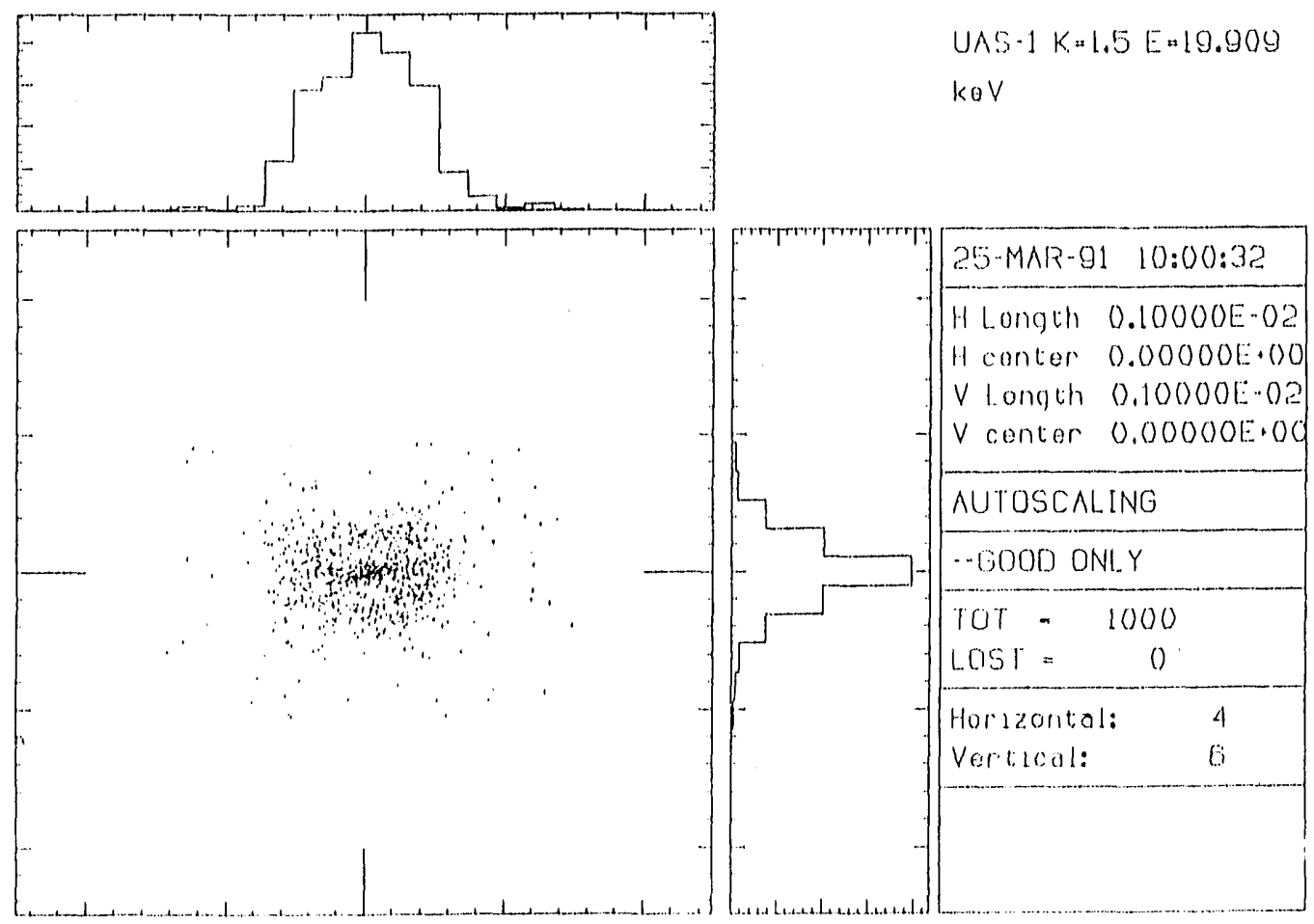

UAS-0 K=1.5E: $=10.000$

kov

25-NAR-91 ()9:55:31

$H$ longth $0.100000 \cdot 02$

$H$ conter $0.000000+00$

$V$ longth $0.10000 \mathrm{E} \cdot 0 \mathrm{O}$

$\checkmark$ cantar 0.000000 .00

AUTOSCALING

- GOOOD ONLY

TOT -1000

LOST $: 0$

Horizontal: 4

Vortical: $B$

25-MAR-199109:58

UAS-1 K. $=1.5 E=19.909$

$\mathrm{k} \theta \mathrm{V}$

25 -MAR-91 10:00:3?

H Longth 0.10000 E.02

H conter $0.00000 \mathrm{E} \cdot 00$

$\checkmark$ l.ongch 0.100000 .0 ?

-

$=$

Fig. UA-21. $\mathrm{K}=1.5$, third harmonic. 
USEROLISK:XUS.SHADOWIBEGIN.DATIB
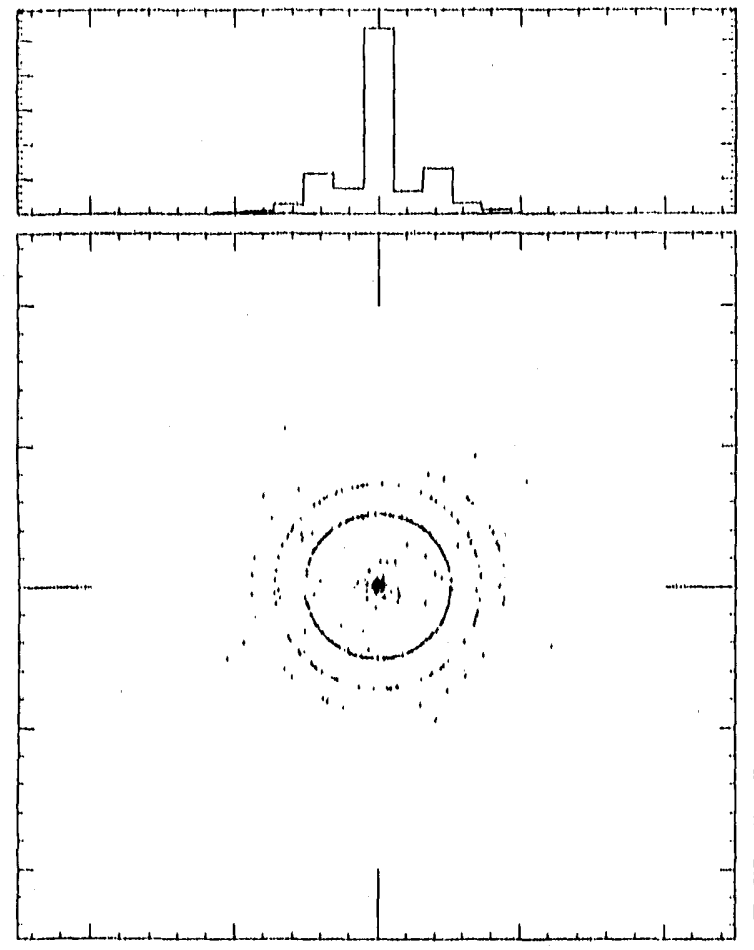

USERAOISK:IXUS.SHAOOWIBEGIN.OATTG
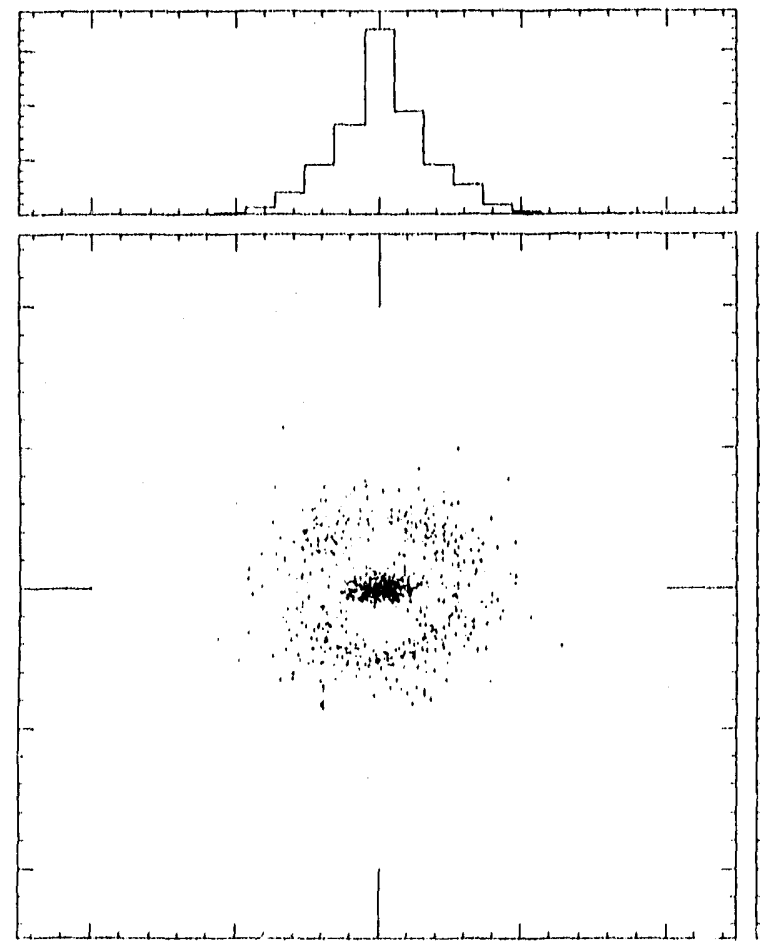

$8-\Lambda P R-199112: 06$

UAS () K=1.AF $1:=1.12 k$

oV

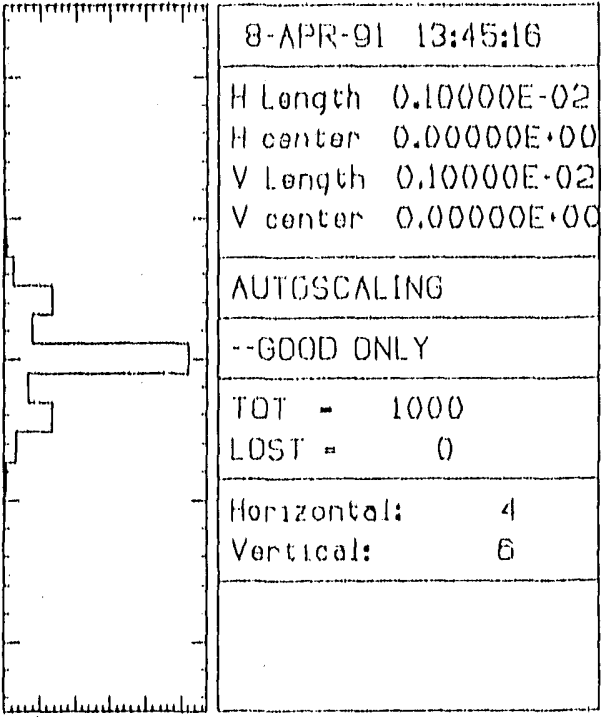

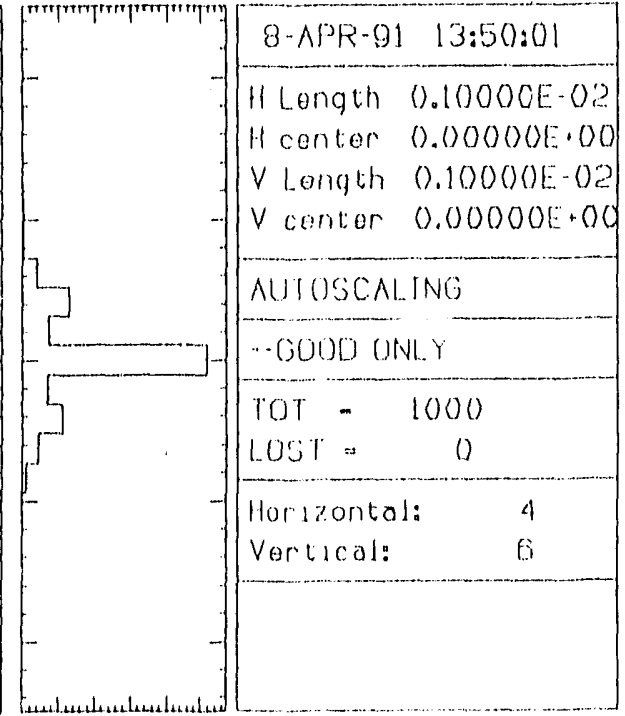

Fig. UA-22. $\mathrm{K}=1.4$, first harmonic. 

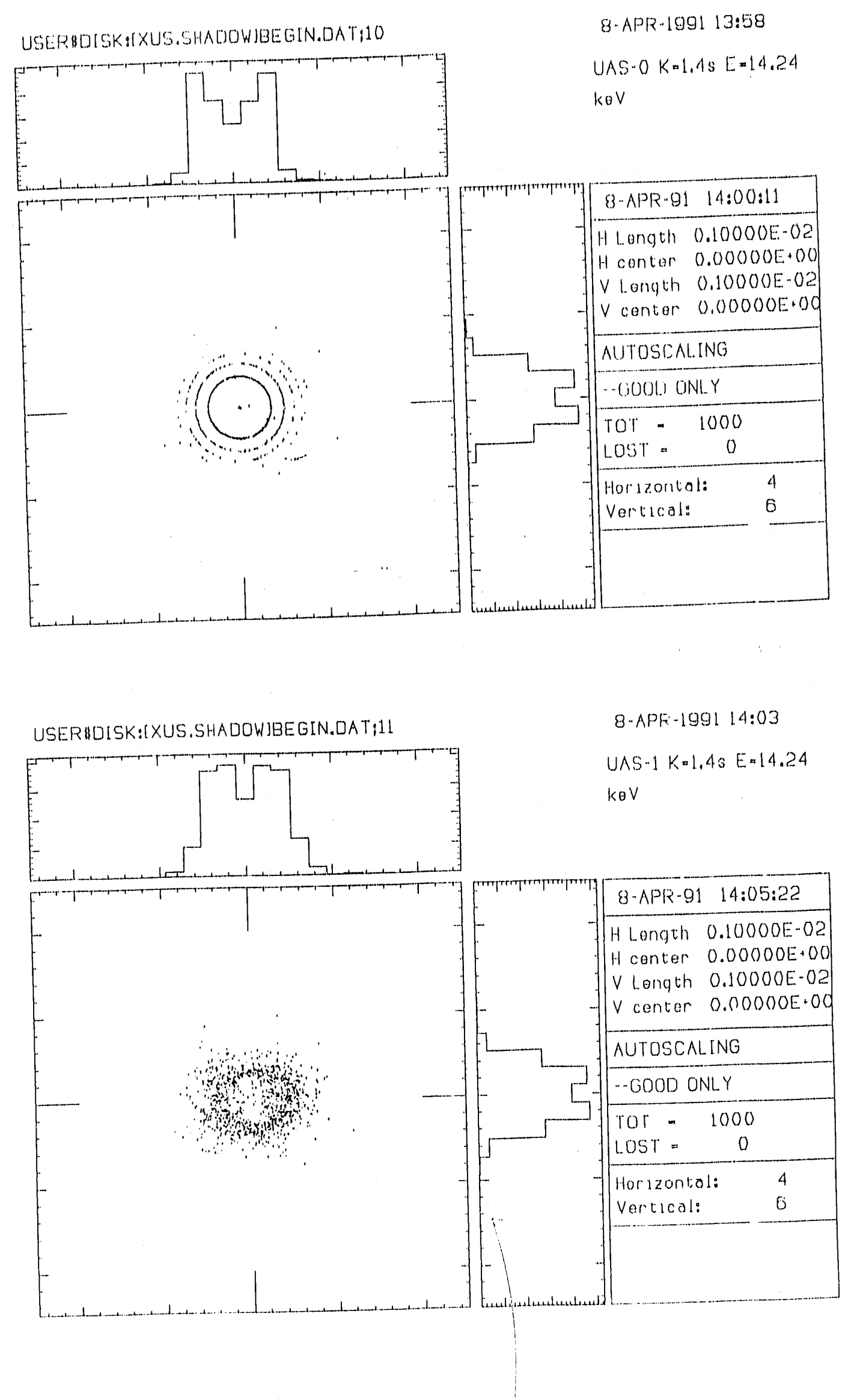

Fig. UA-23. $\quad K=1.4$, second harmonic. 
USERHDISK:IXUS. SHADOWIBEGIN.DAT:L
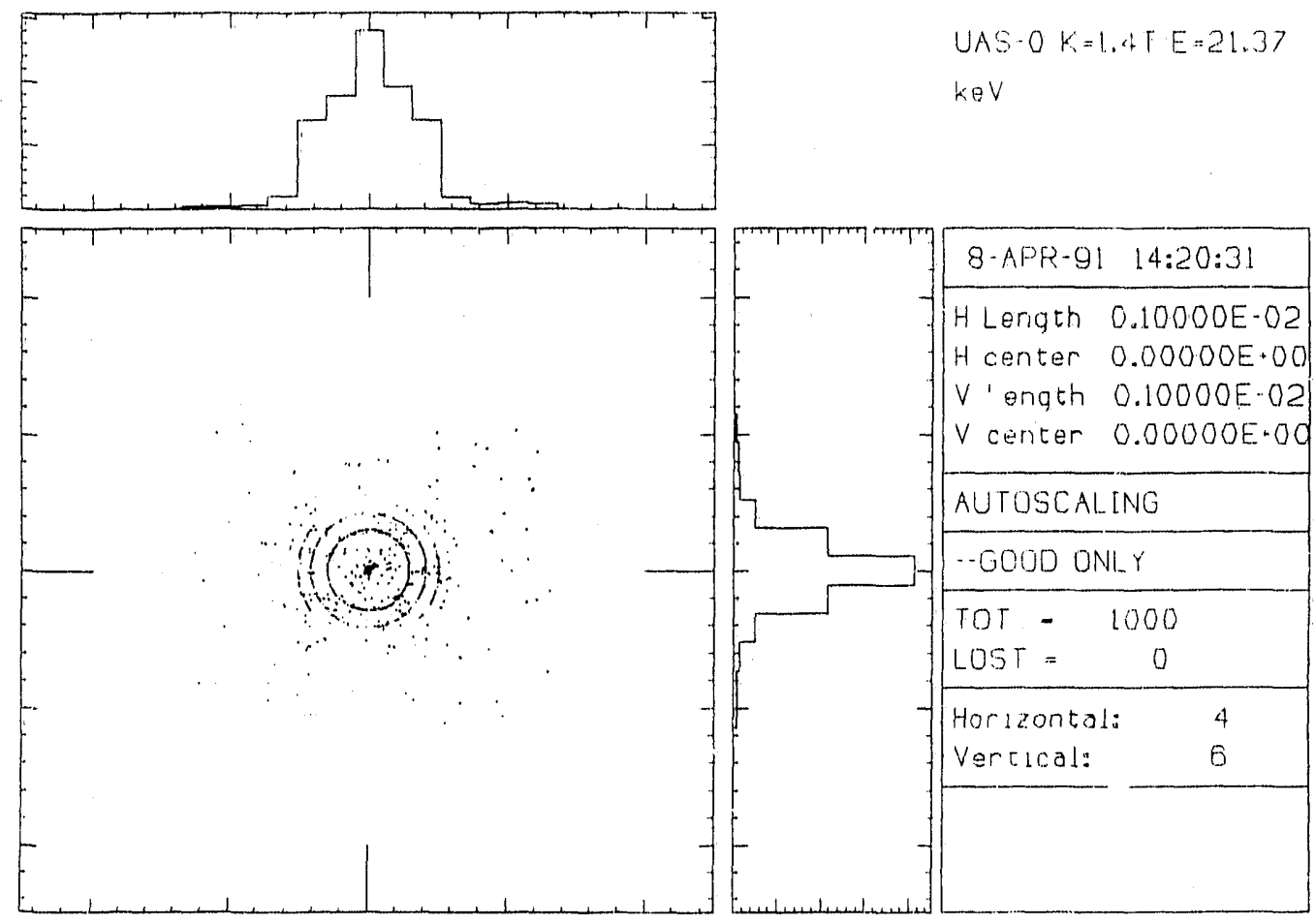

USER\&DISK:[XUS.SHADOW]BEGIN.OAT:Z
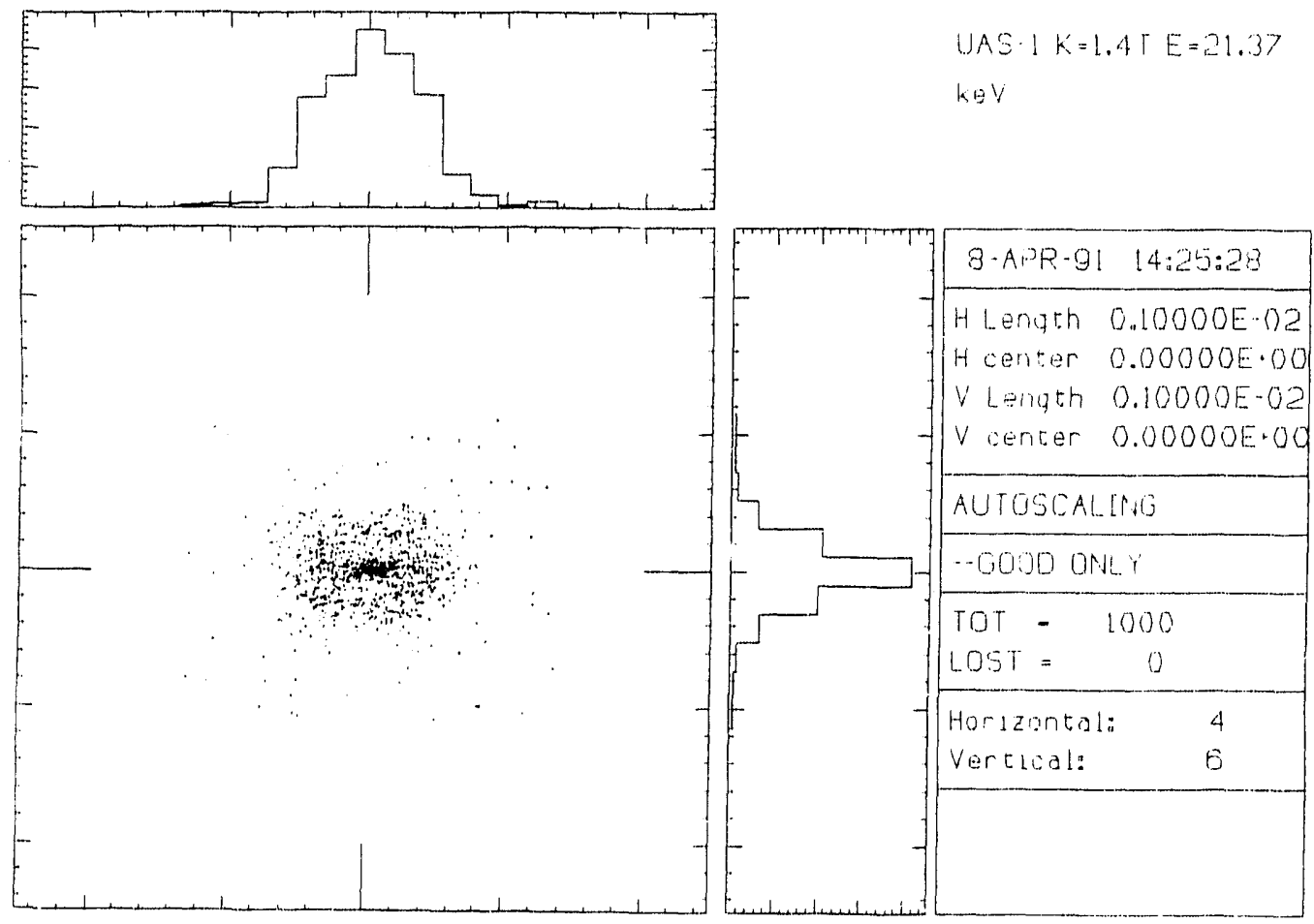

$U A S \cdot O K=1.4 T E=21.37$

$\mathrm{keV}$
8-APR-1991 14:22

USS.1K=1.4TE=21.37

k日V

Fig. UA-24. $\mathrm{K}=1.4$, third harmonic. 

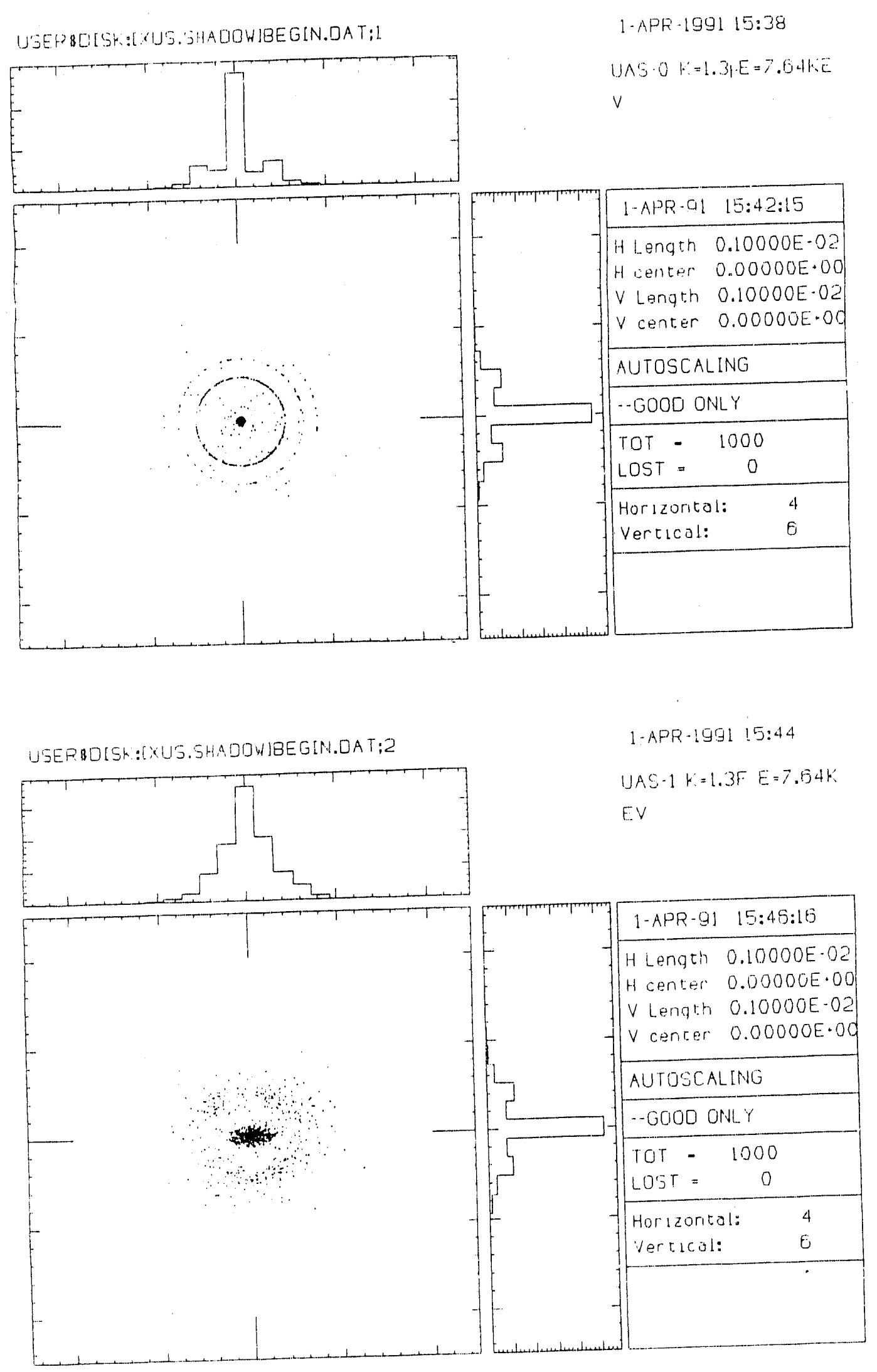

Fig. UA-25. $\mathrm{K}=1.3$, first harmonic. 

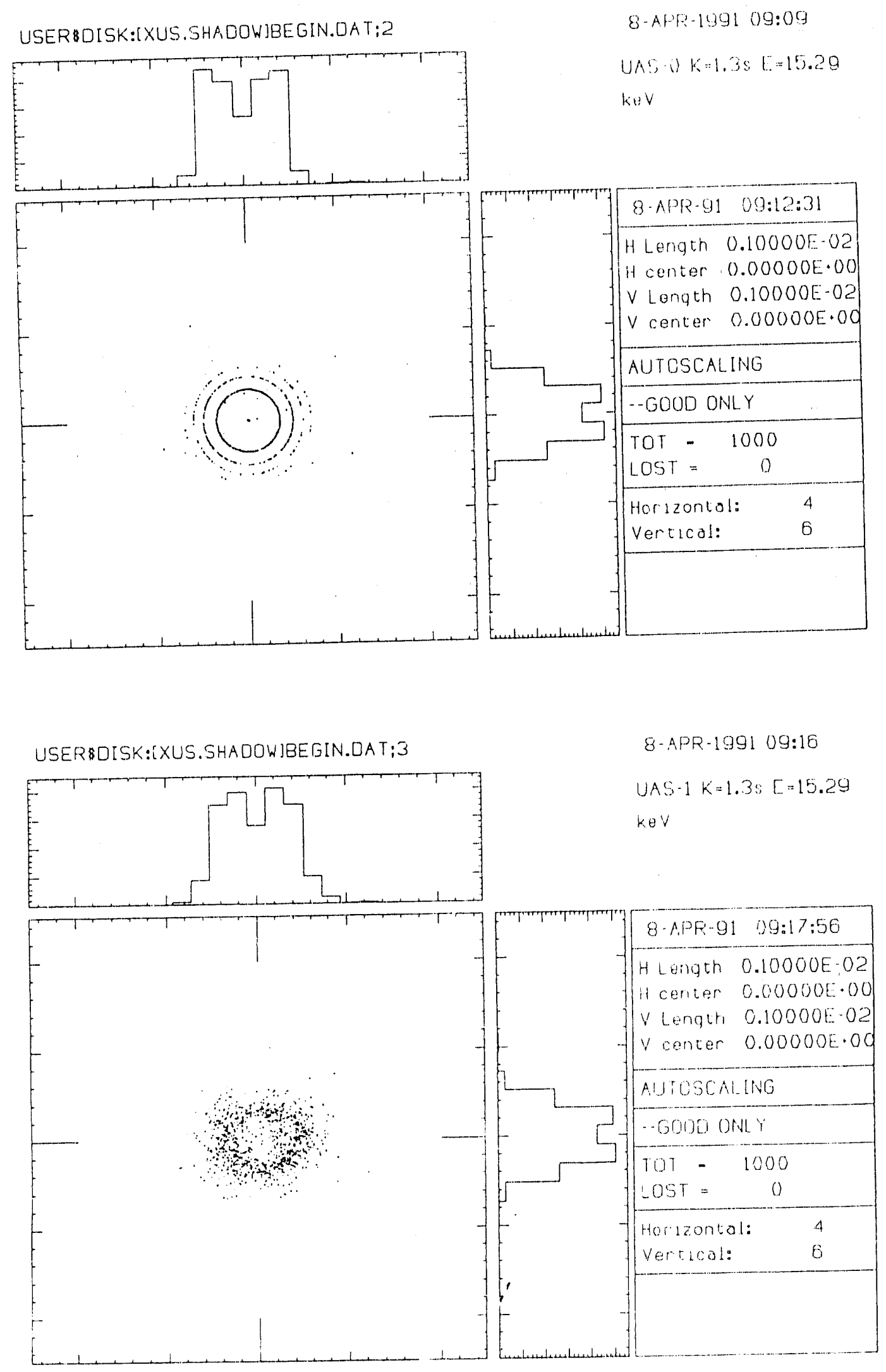

Fig. UA-26. $\quad \mathrm{K}=1.3$, second harmonic. 


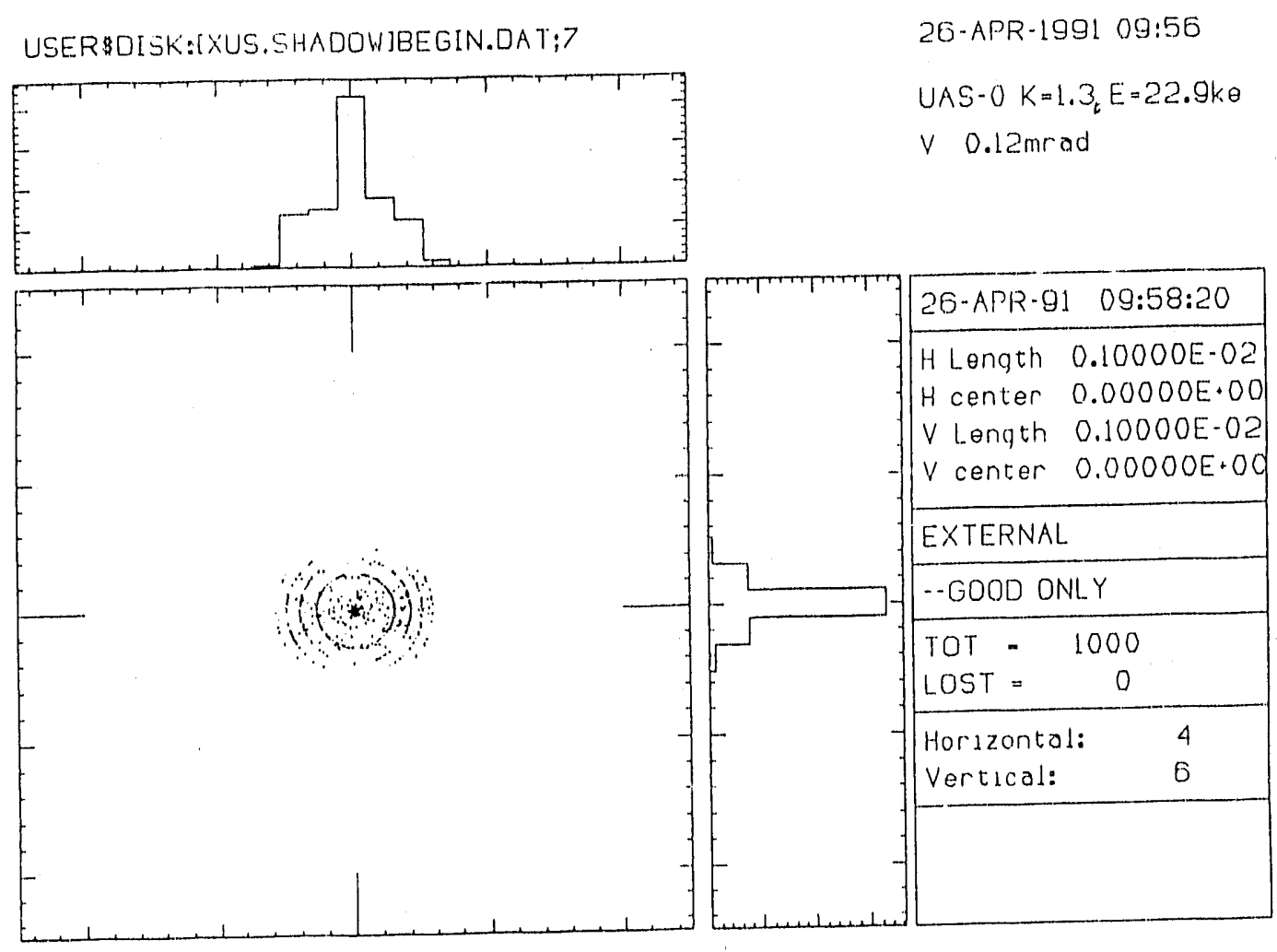

USER8DISK:IXUS.SHADOW]BEGIN.DAT;8
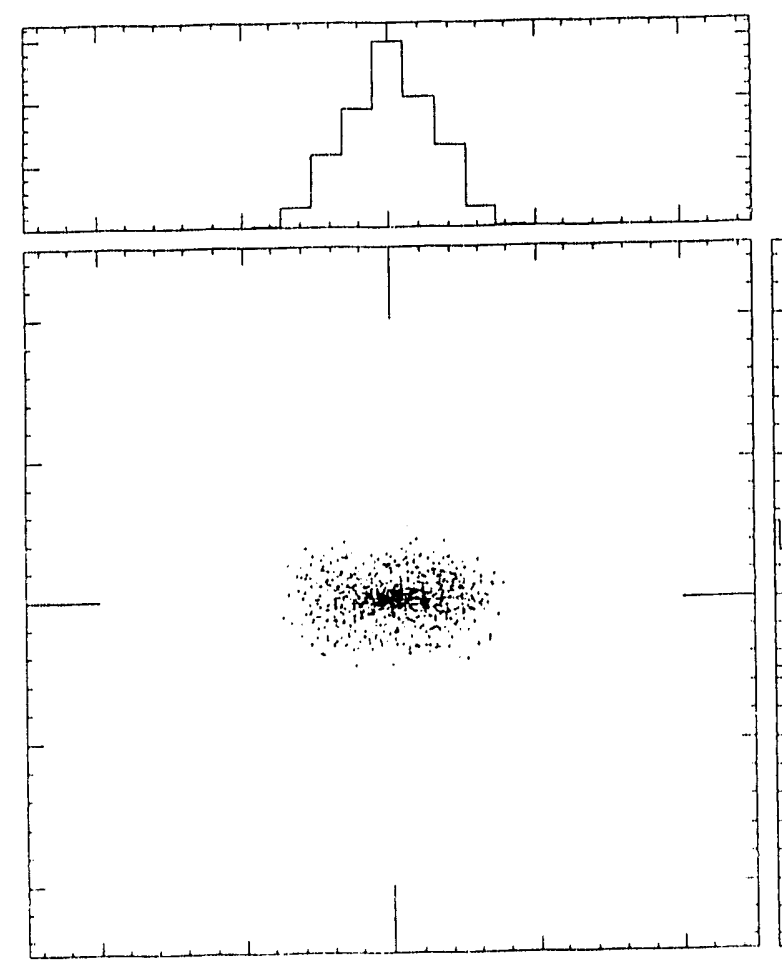

26-APR-1991 10:00

UAS $-1 K=1.3, E=22.9 k \theta$

$\checkmark 0.12 \mathrm{mrad}$

Fig. UA-27. $\mathrm{K}=1.3$, third harmonic.

41

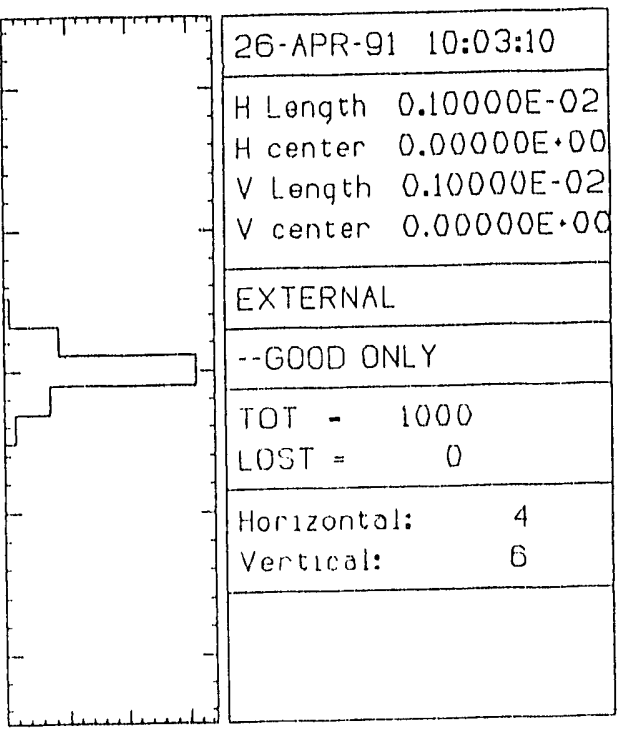


USERBDISK:IXUS.SHAOOWIBEGIN.DA T:13
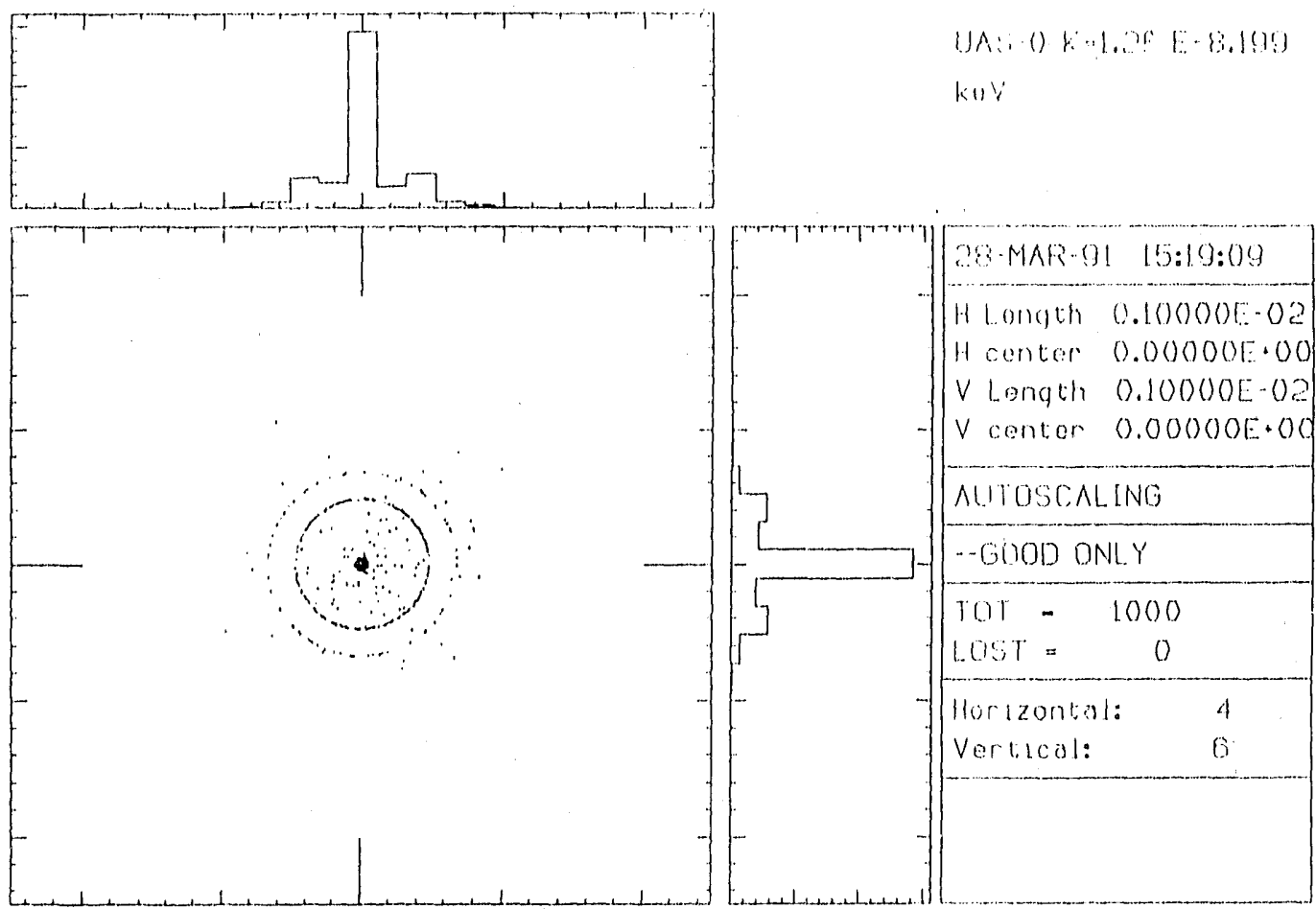

USEFULISK:[XUS.SHAOOW]BEGIN.DATI14
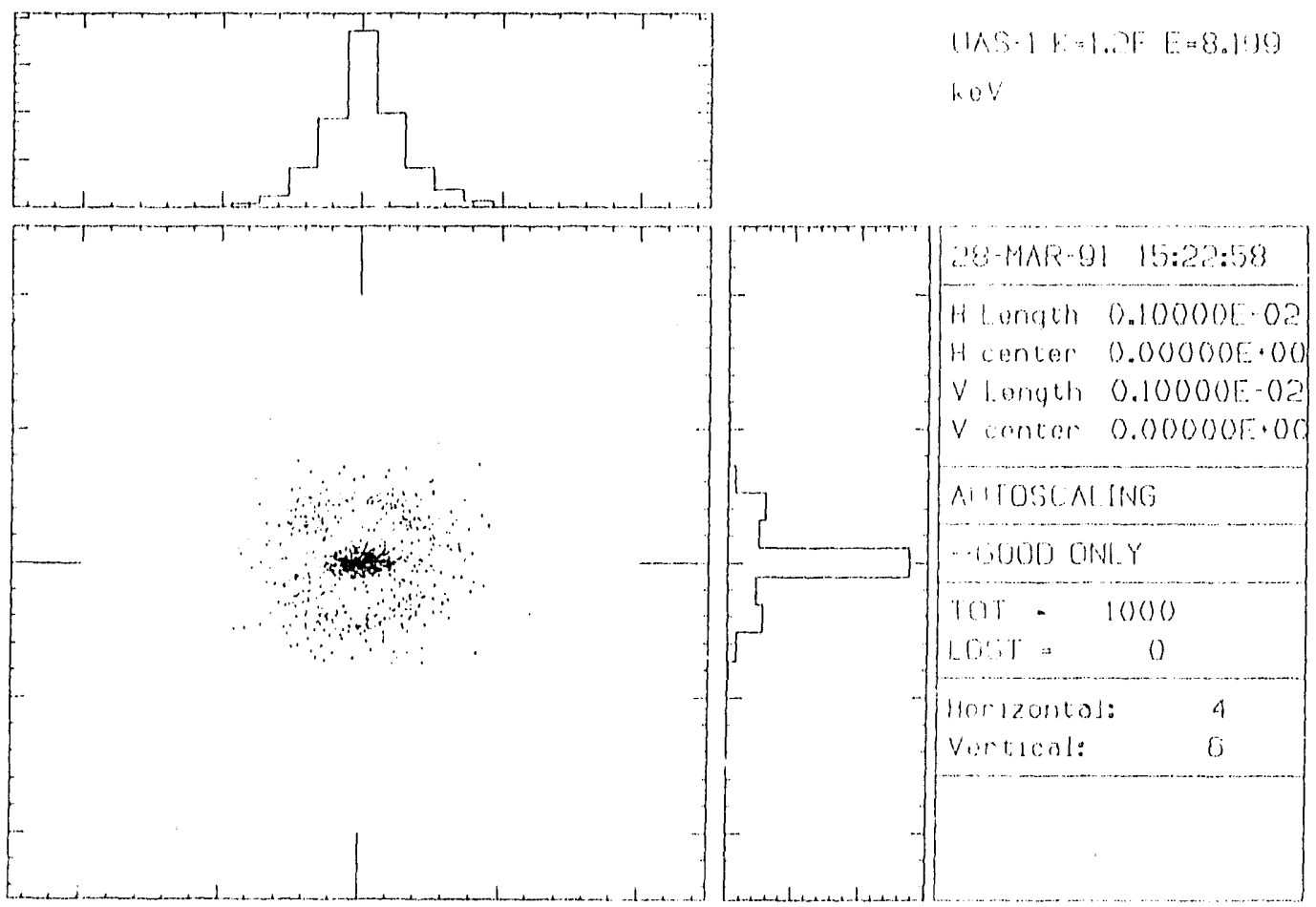

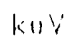

UA: () $8 \div 1.298-8.1(1)$

$28-M A R \cdot 0 ! \quad 15: 10: 00$

Vantor $0.000000+00$

ATOSCALINO

- GGOD DNL.Y

TOT -1000

lorlzontal: $\quad 4$

vertical:

?5: MNE-1431 15:?

$(1 \mathrm{~S} 5 \cdot 1 \%=1.2 \%=8.8 .190$

key

Fig. UA-28. $\quad \mathrm{K}=1.2$, first harmonic. 
USER BISK:[XUS.SHADOW]BEGIN.DAT:I
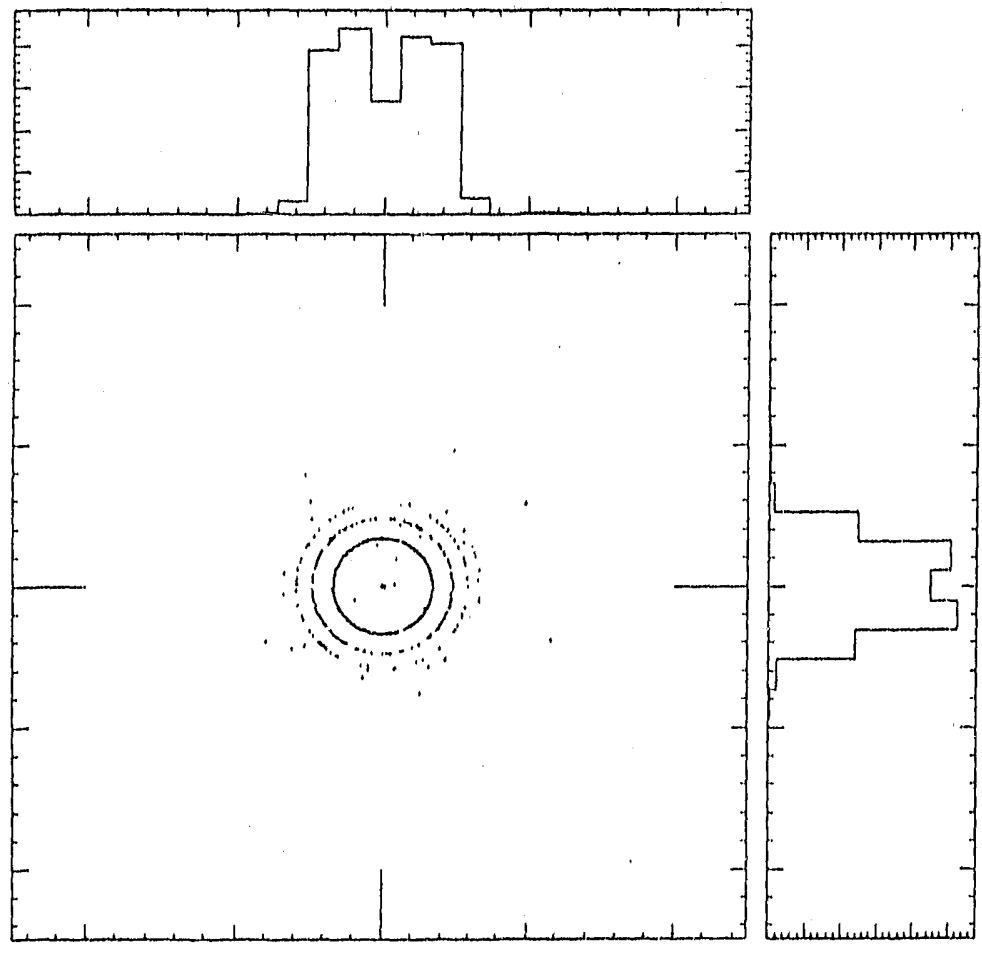

USERBDISK:[XUS.SHADOW]BEGIN.DAT:2
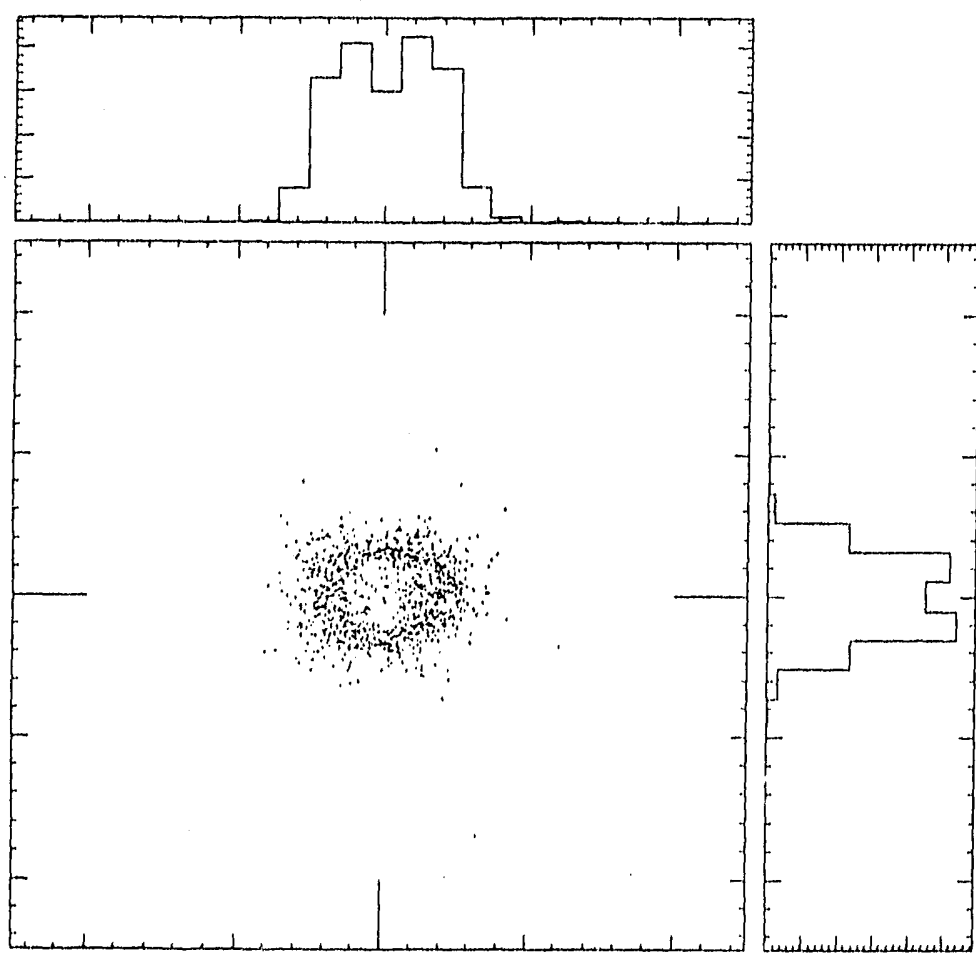

28-MAR-1991 15:31

UAS-O $K=1.25 \quad E=16.39$

$8 \mathrm{keV}$

28-MAR-91 $\quad 15: 33: 25$

H Length $0.10000 E-02$

$H$ center $0.00000 E+00$

$V$ Lenqth $0.10000 E-02$

$V$ center $0.00000 E+00$

AUTOSCALING

-GOOD ONLY

TOT - 1000

LOST $=0$

Horzzontol: 4

Vertical: 6

28-MAR-1991 15:35

UAS-1 $K=1.2$ S $E=16.39$

$8 k_{\theta} V$

28-MAR-91 15:36:53

H length $0.10000 E-02$

$H$ center $0.00000 E+00$

$V$ Length $0.10000 E-02$

$V$ center $0.00000 E+00$

AUTOSCALING

- -GOOD ONLY

TOT - 1000

LOST $=$

Horizontal: 4

Vertical: $\quad 6$

Fig. UA-29. $\mathrm{K}=1.2$, second harmonic. 
USERBDISK:IXUS.SHADOWIBEGIN.UAT;5
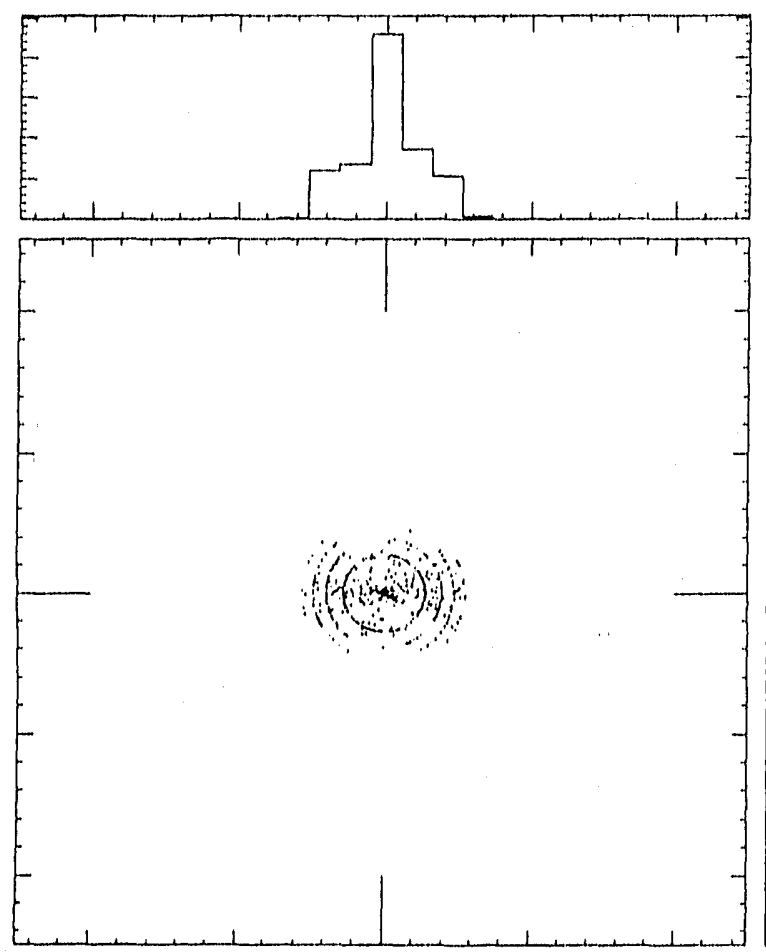

USER\&DISK:[XUS.SHADOW]BEGIN.DAT;6
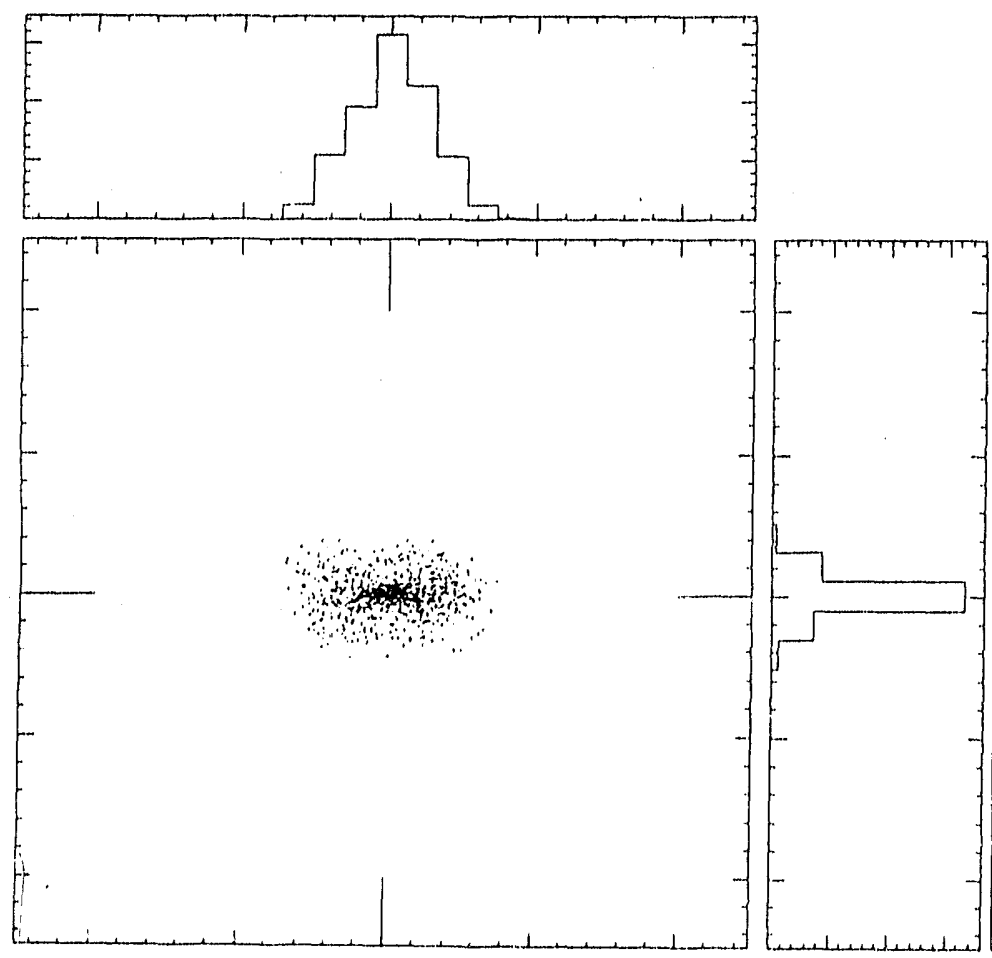

26-APR-1991 09:34

$U A S-0 K=1.2, E=24.6 k \theta$

$\checkmark 0.012 \mathrm{mrod}$

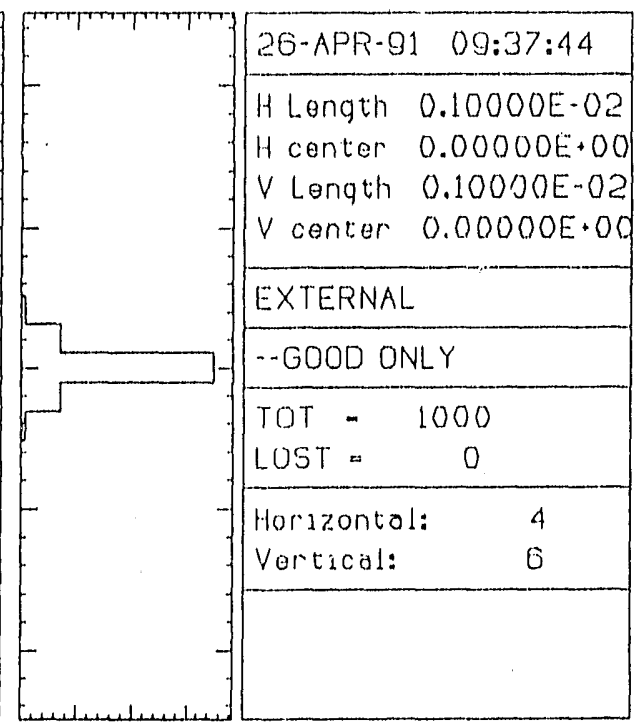

26-APR-199109:40

$U A S-1 K=1.2, E=24.6 k \theta$

V $0.12 \mathrm{mrad}$

1

26-APR-91 09:44:25

H Length $0.10000 E-02$

$H$ center $0.00000 E+00$

$V$ Length $0.10000 E-02$

$V$ center $0.000000+00$

EXTERNAL

-GODO ONLY

TOT - 1000

$1.05 T=0$

Horizontal: 4

Vertical: $\quad 6$

Fig. UA-30. $\mathrm{K}=1.2$, third harmonic. 
USERBDISK:[XUS.SHAOOWIBEGIN.DAT:7
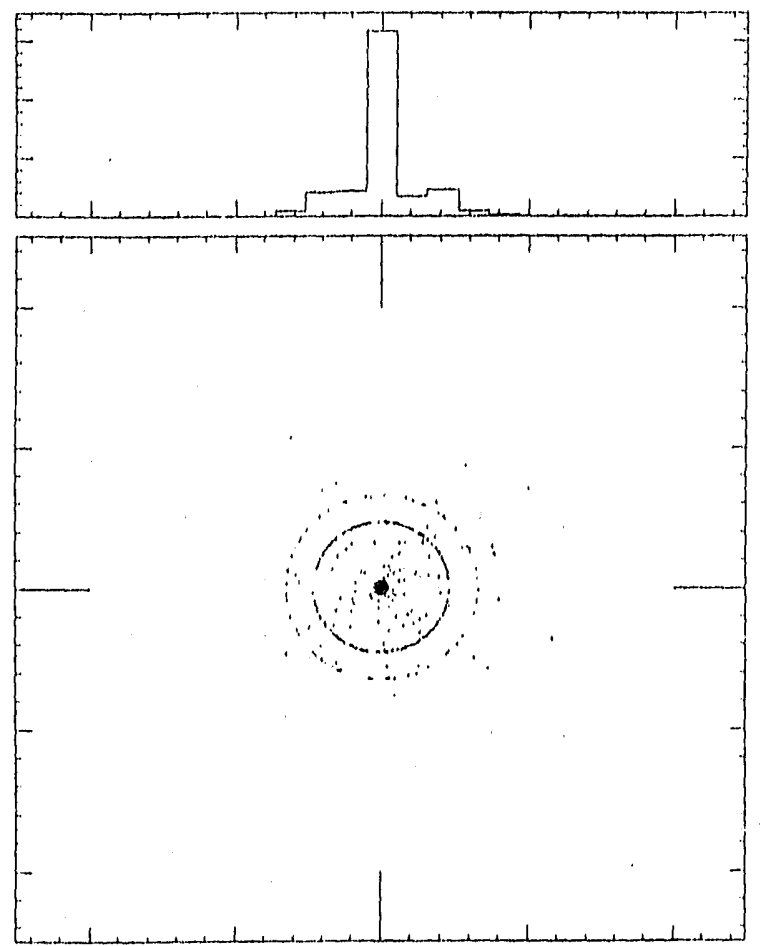

USERBDISK:[XUS.SHADOW]BEGIN.DAT:8
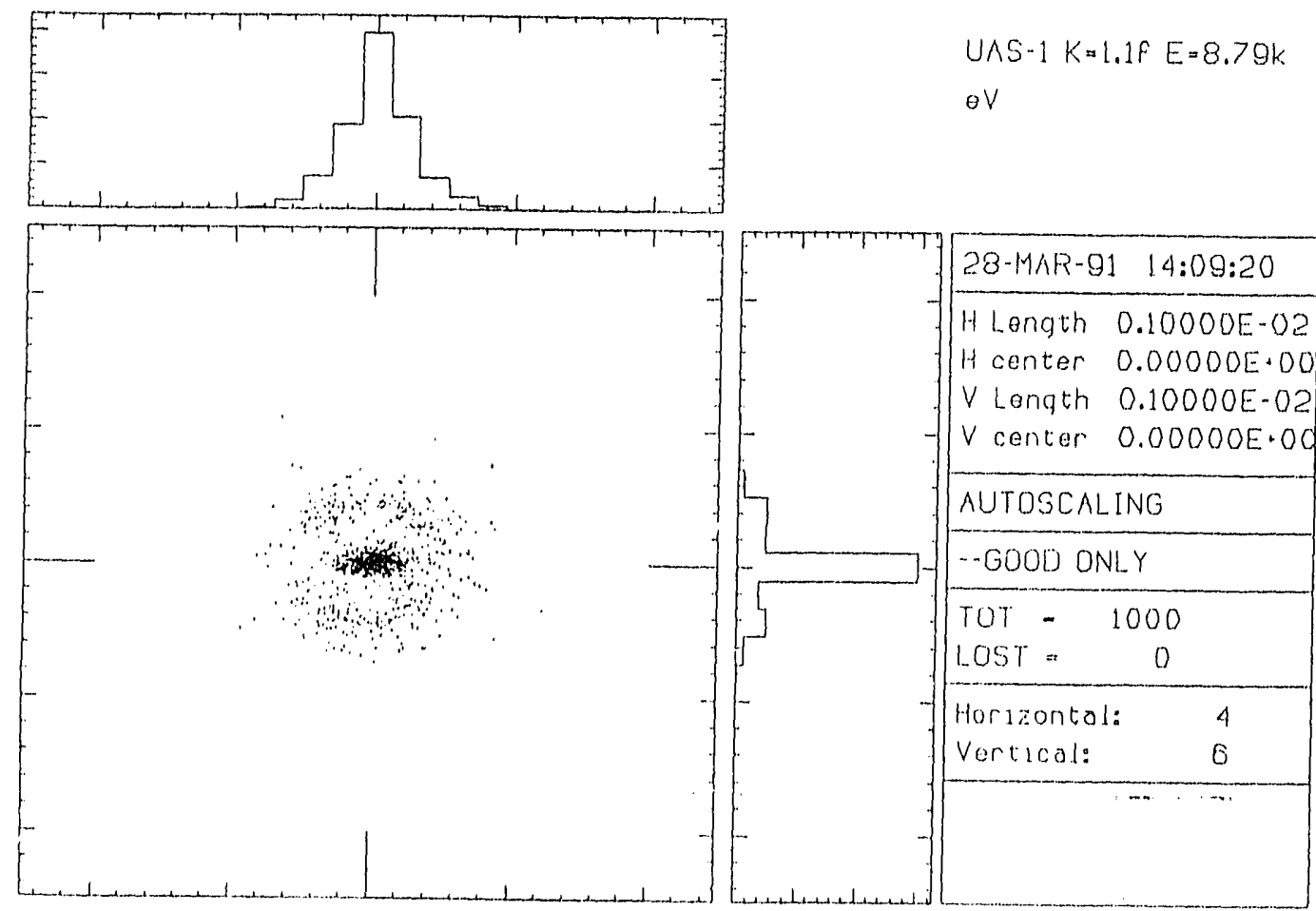

$U A S-O K=1.1 F E=8.79 K$

oV
23-MAR-1991 14:03

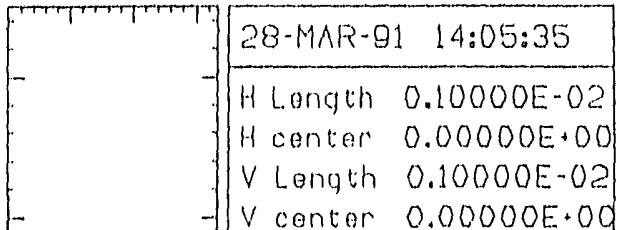

AUTOSCALING

- GOOD ONLY

TOT -1000 $\operatorname{LOST}=\quad 0$

Horizontal: 4

Vertical: 6

28-MAR-1991 14:07

$U \wedge S-1 K=1.1 \mathrm{f} E=8.79 \mathrm{~K}$

$\theta \mathrm{V}$

Fig. UA-31. $\quad \mathrm{K}=1.1$, first harmonic. 
USERHOISK:IXIJS.SHAOOWIBEGIN.DATIO

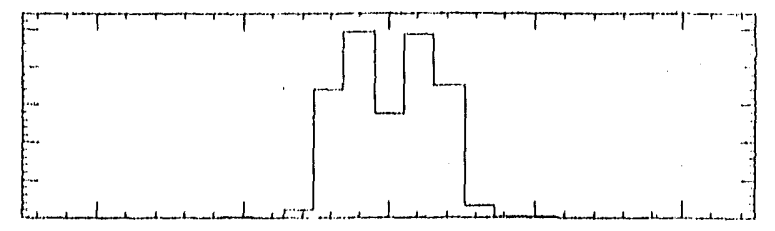

USERBOLSK:IXUS.SHAOOWIBEGIN.OAT:10
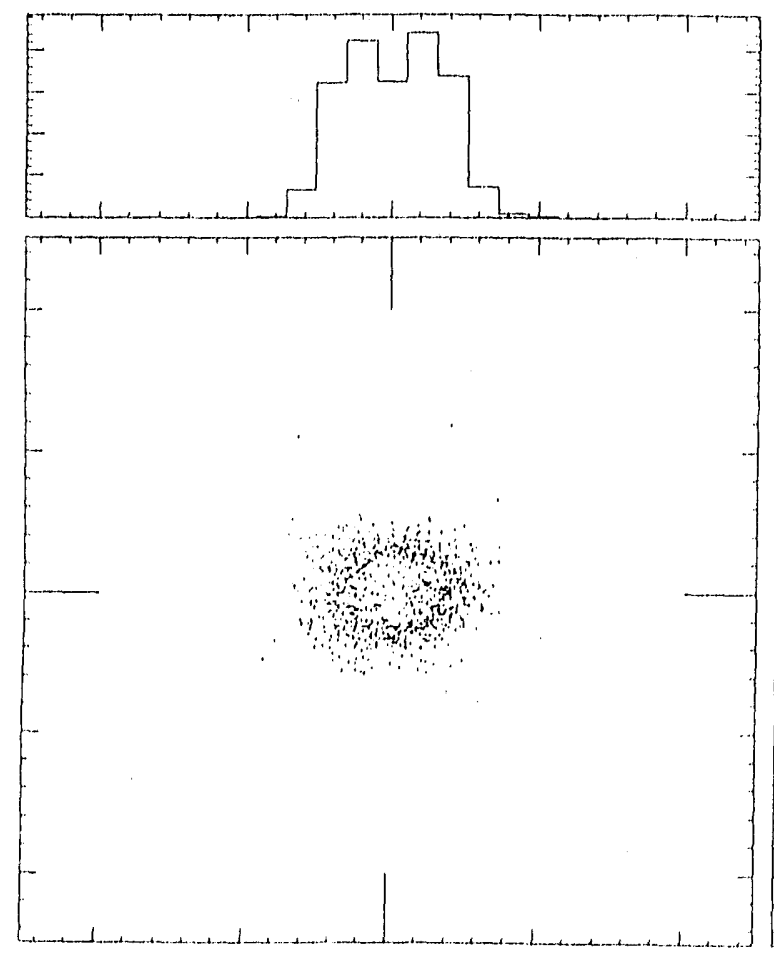

28 - HAR-1.991 14:183

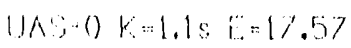

kov

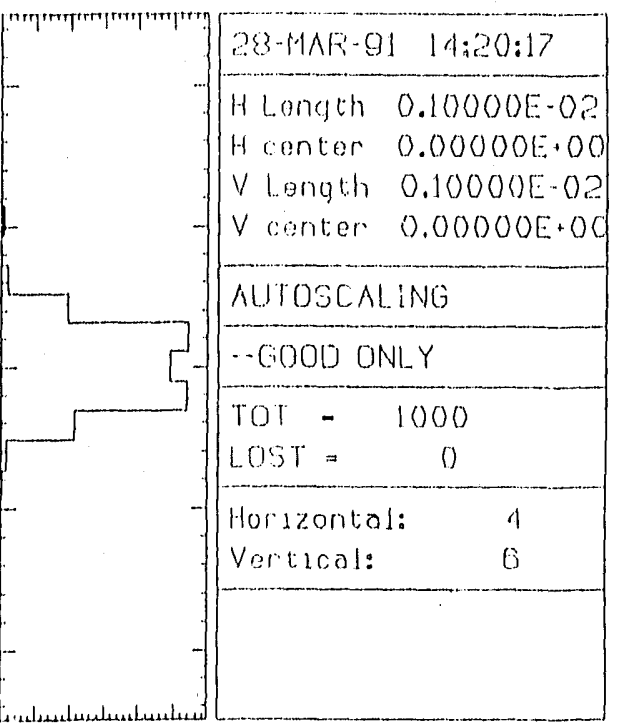

IS-MAR-1991 14:24 UAS-1 K.1.1s E:-1\%.57

k.1. V

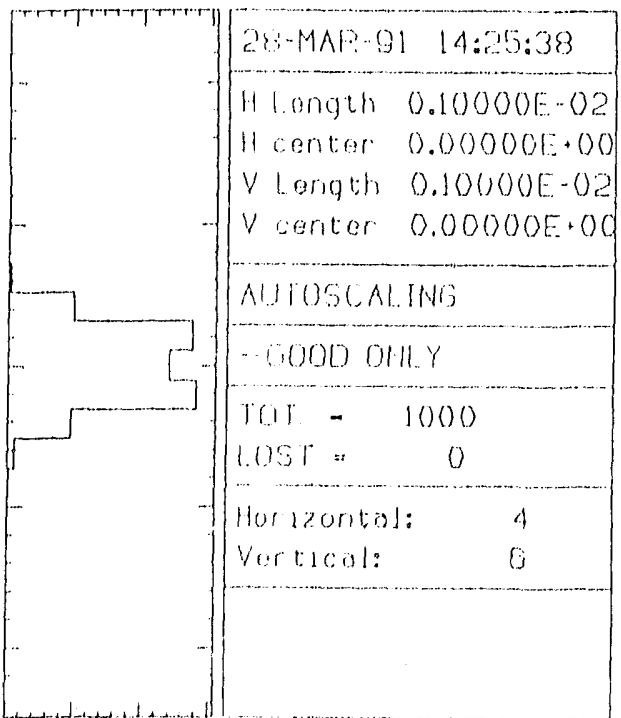

Fig. UA-32. $\mathrm{K}=1.1$, second harmonic. 
USERYDISK:IXUS.SHAOOWIBEGIN.DAT/3

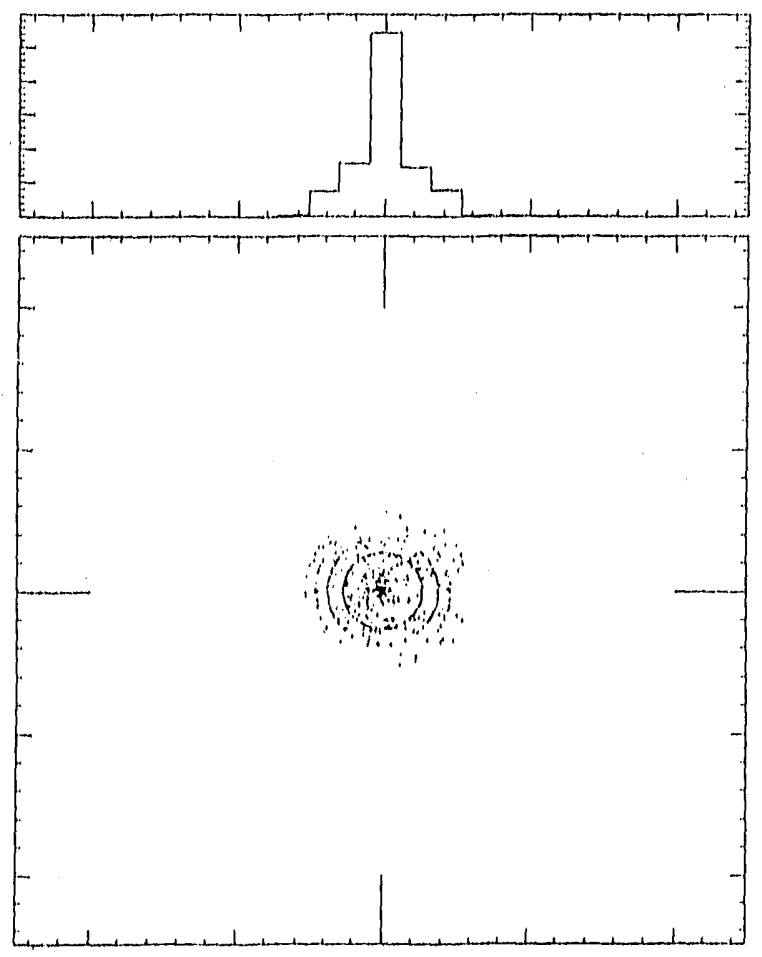

USERBDISK:IXUS.SHADOW]BEGIN.DAT;4
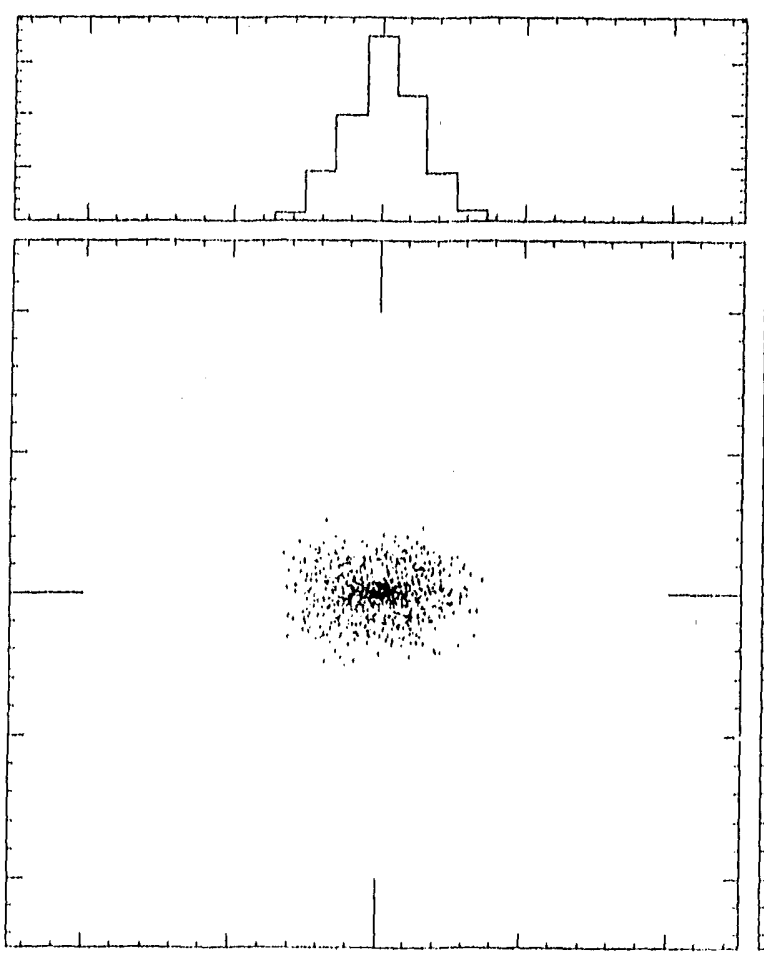

$26-A P F-199109: 05$

UAS-0 K=1.1, $F=26.36 \mathrm{~K}$

ov $0.12 m r$ od

20-APR-91 09:13:10

H Length 0.10000 E-02

H centur 0.00000E.00

$V$ Length $0.100000 \mathrm{E}-02$

$V$ centar $0.00000 E+00$

AUTOSCAL.ING

-GOOD ONL. Y

TOT - 1000

LOST $=0$

Horszontal: 4

Vertical: $\quad 6$

26-APR-1991 U9:15

UAS-1 $K=1.1, E=26.36 \mathrm{~K}$

ov $0.12 \mathrm{mrod}$

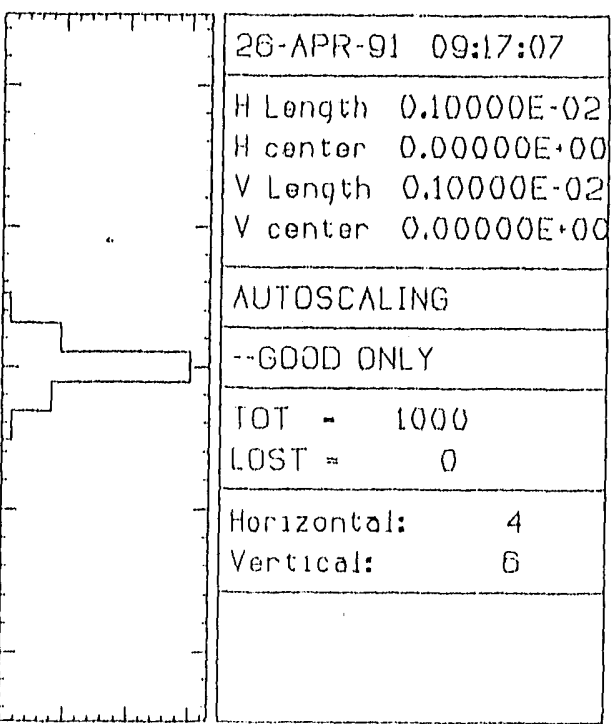

Fig. UA-33. $\mathrm{K}=1.1$, third harmonic. 


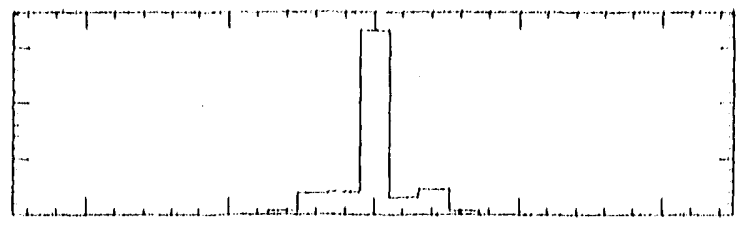

UA: () $K=1 .()+\cdots(1.41 .0) \mathrm{V}$

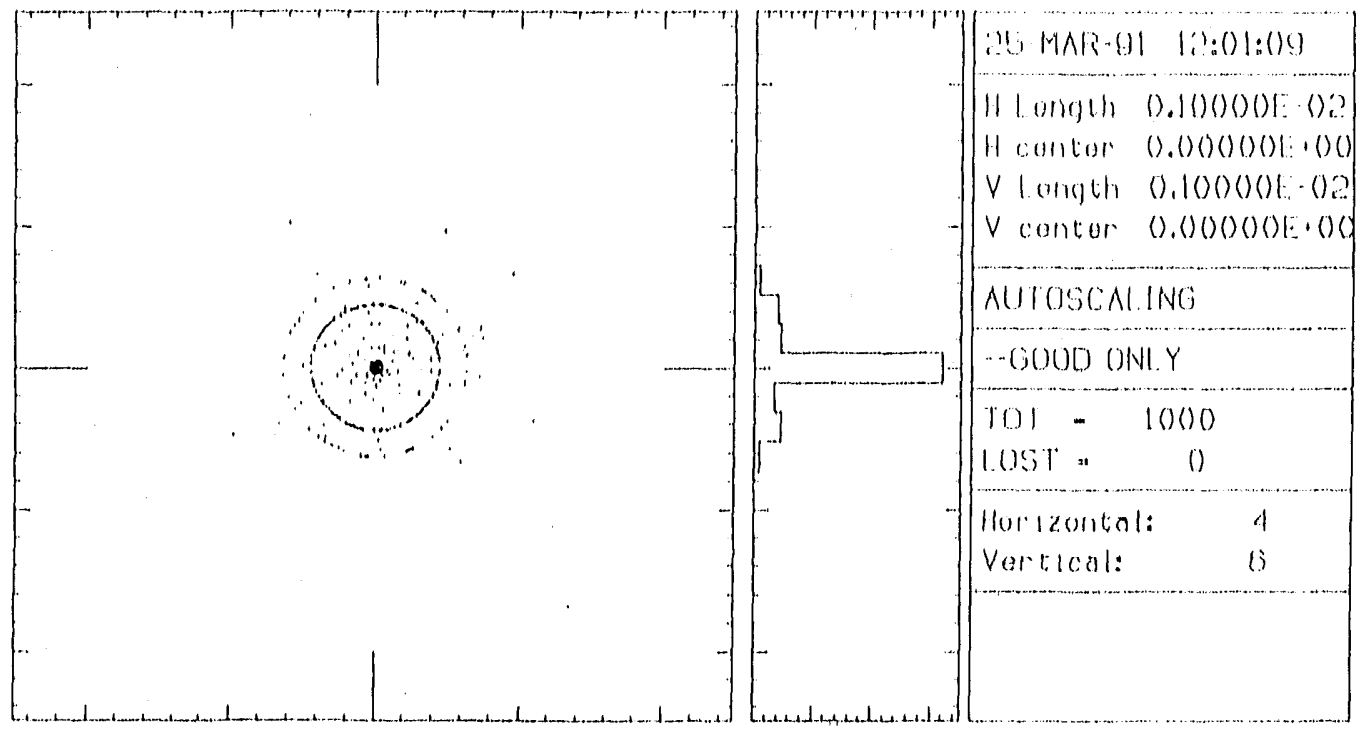

USERBDISK:LXUS.SHAOOWIBEGIN.OAT:S
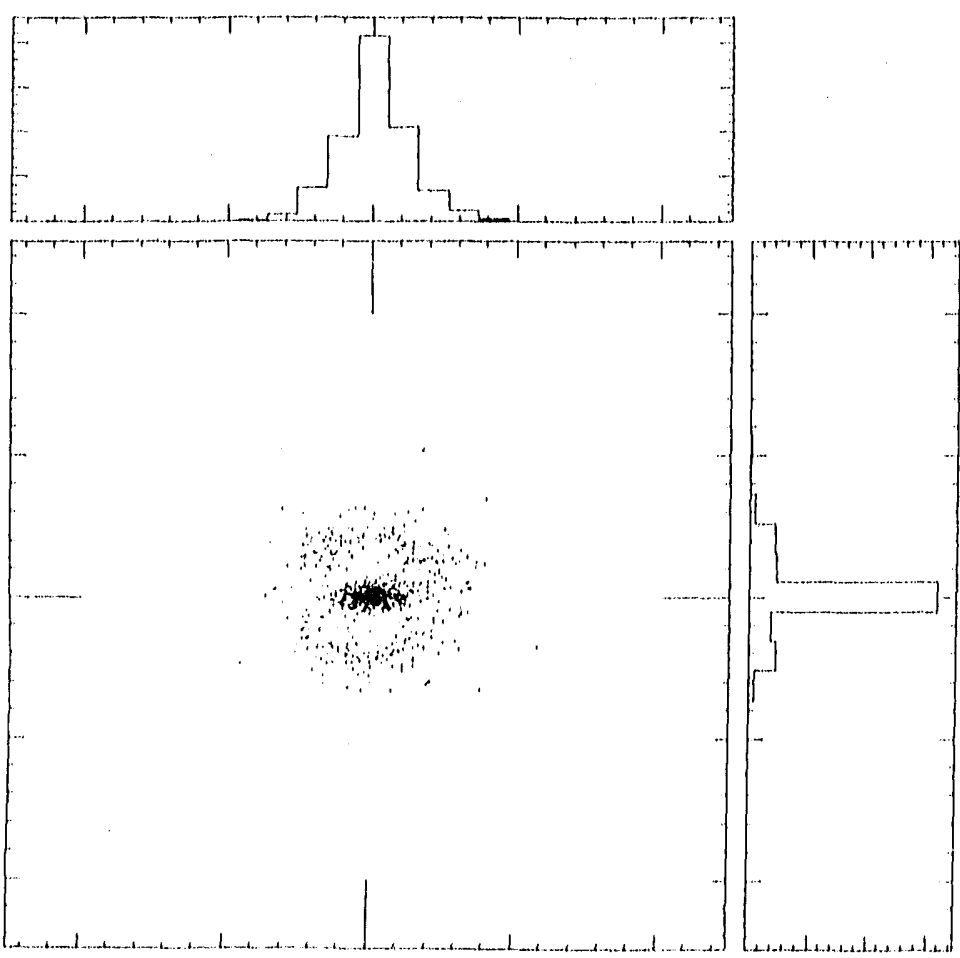

20.MAR-10911 11:55

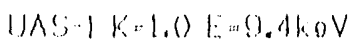

25 MARi-01 11:5\%:0\%

H Longth $0.10(0)()(0) \cdot 02$

$H$ conter $(0.0)(0)(0) \cdot(0)$

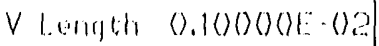

$\checkmark$ coeritor $0.00(0)(0) \cdot 0(0)$

AUTOSOALINE

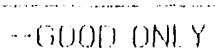

$101-2000$

$10151=0$

Horrantal: A

Vorticol: 6

Fig. UA-34. $\mathrm{K}=1.0$, first harmonic. 
USERHOISK:IXUS,SHAOOWIEZCOLN.DATII
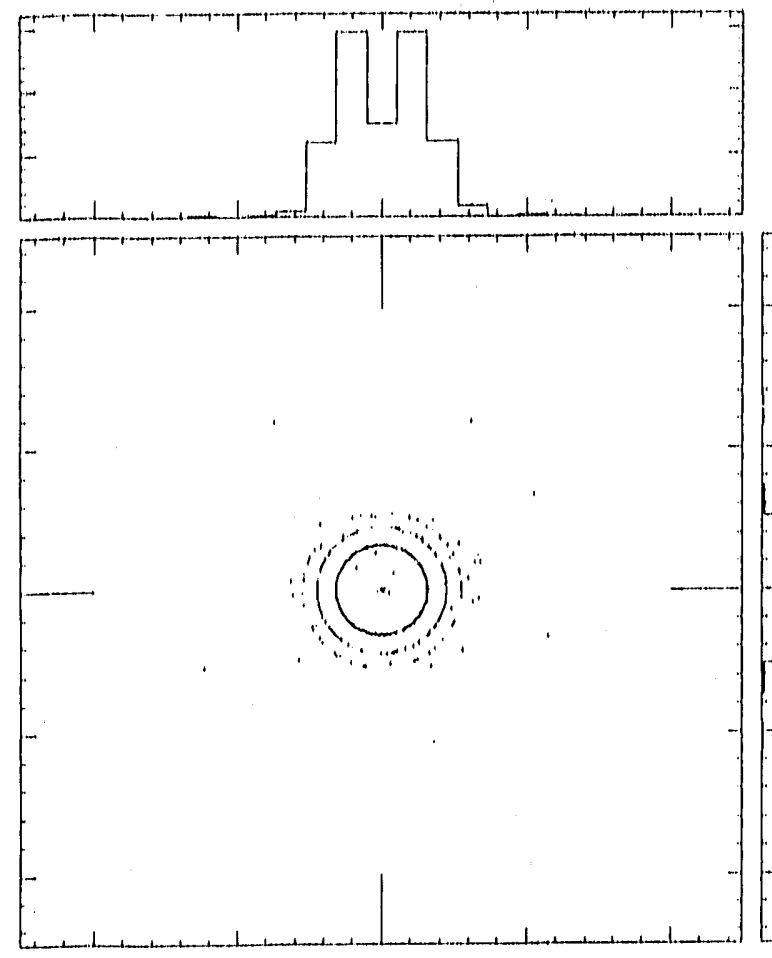

USERBDISK:IXUS,SHADOWIBEGIN.DAT:2
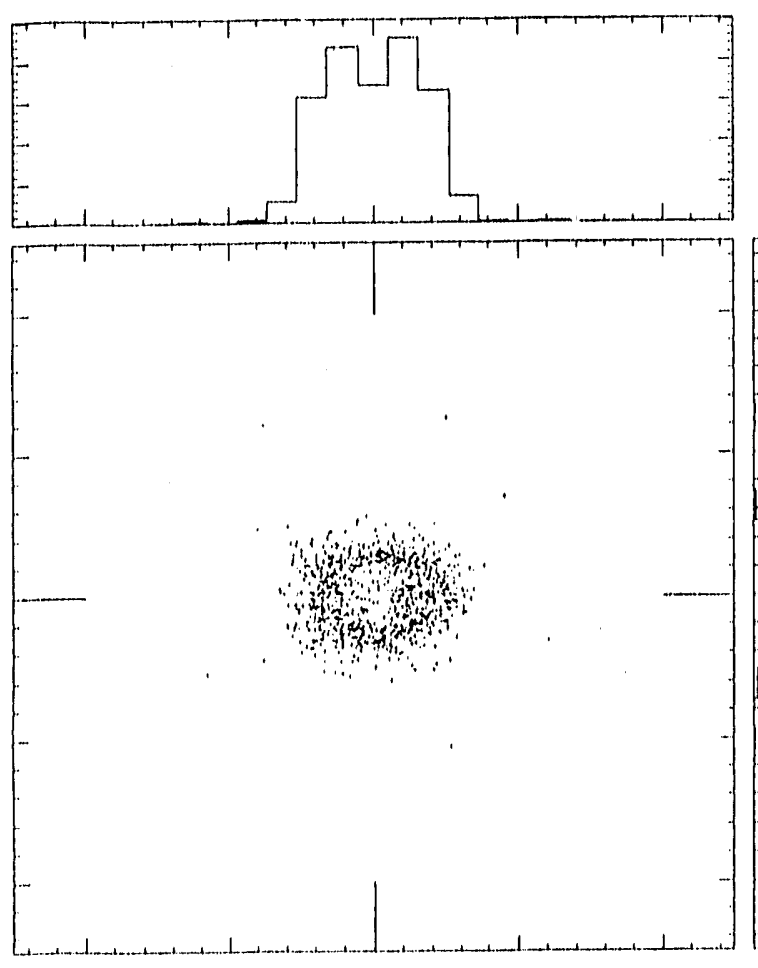

$25 \cdot M A R-1991 \quad 13: 57$

UAS () $K=1.0) E 18.8 \mathrm{ko}$

$\mathrm{v}$

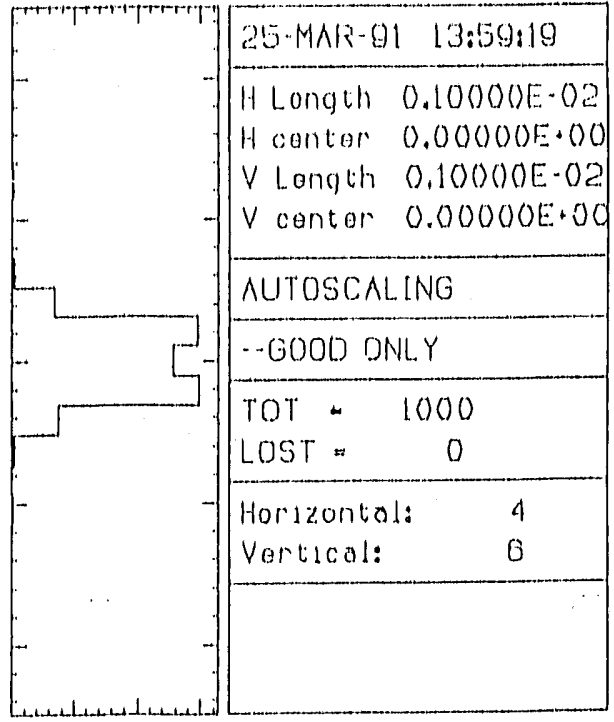

25.MAR-1991 14:02

UAS-1 K.1.0 E.18.8Ko

$\checkmark$

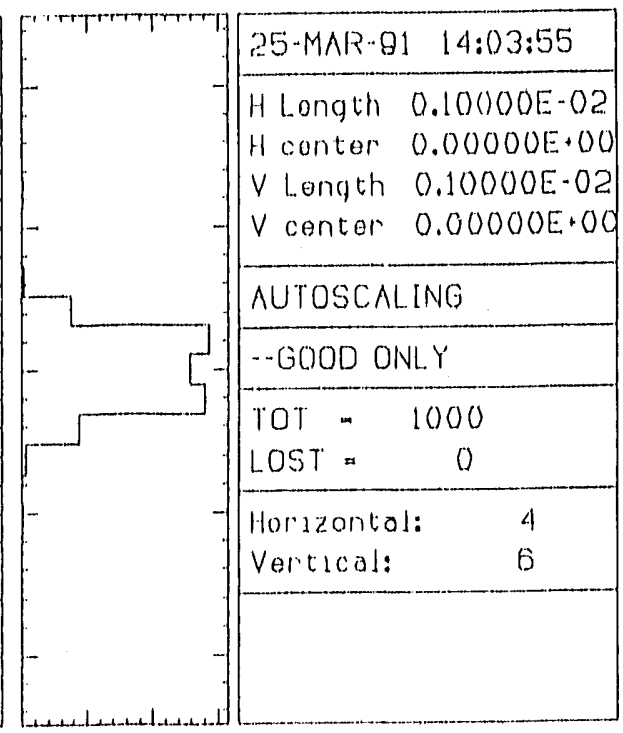

Fig. UA-35. $\quad \mathrm{K}=1.0$, second harmonic. 
USERHOISKGXUS, SHAOOWIBEOLIN.OATR
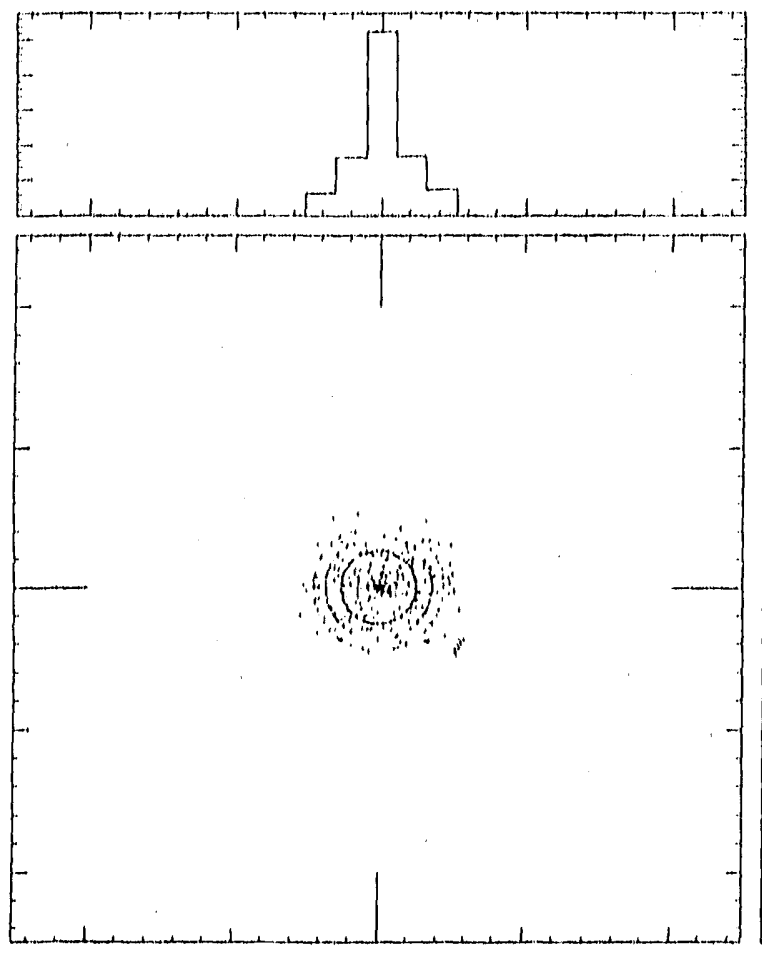

USERBDISK: XUS.SHADOWIBEGIN.OAT;1

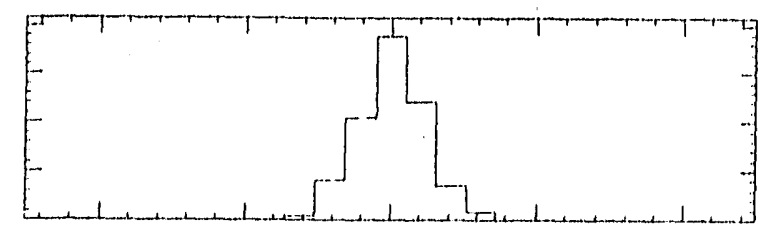

$26-A P R-19911 \% 27$

UAS-1 Kal, E-28.2kOV

$0.12 \mathrm{mrad}$

Fig. UA-36, $\mathrm{K}=1.0$, third harmonic. 
USERHDISK:IXUS.SHADOWIBEGIN.DATII
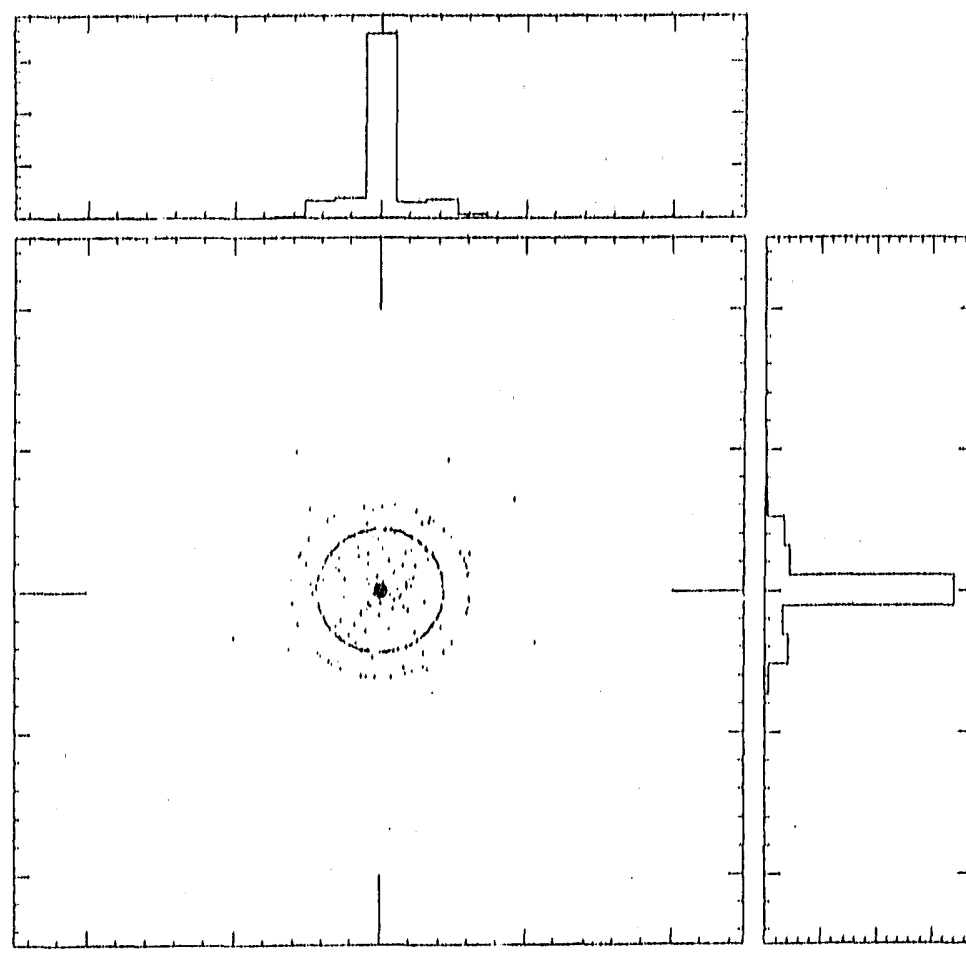

28 MAR-199108159

UAS.0 K.0.9F E. $=10.04$

kov
28-MAR-91 00:01:01

H Longth 0.10000E-02

H center 0.00000 E.00

$V$ Length $0.10000 E-02$

$\checkmark$ center $0.00000 E \cdot 00$

AUTOSCALING

-GOOD ONLY

TOT - 1000

LOST $=0$

Horizontal: 4

Vertical: 6

28-MAR-199109:03

$U A S-1 K=0.9 \% E=10.04$

$k \theta V$

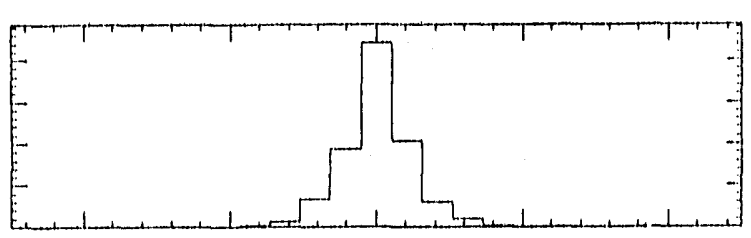

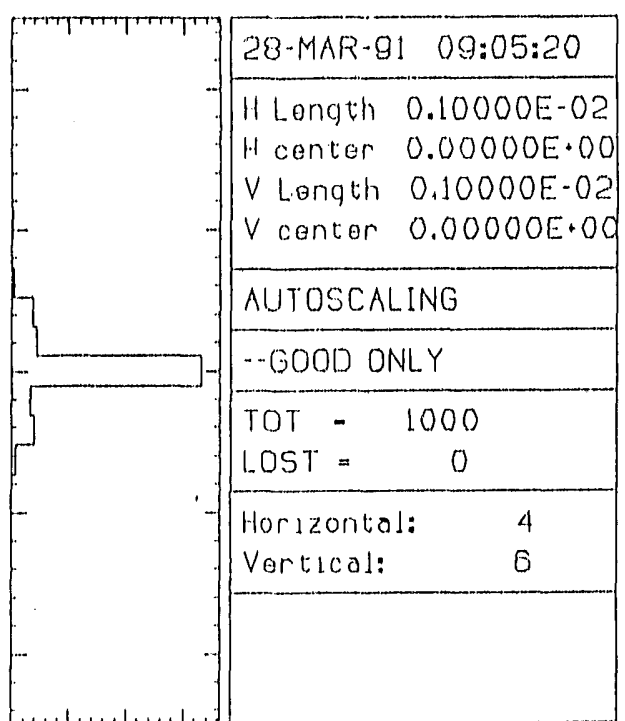

Fig. UA-37. $\mathrm{K}=0.9$, first harmonic. 
USERBDISK:LXUS.SHADOWIBEGIN.DAT:3
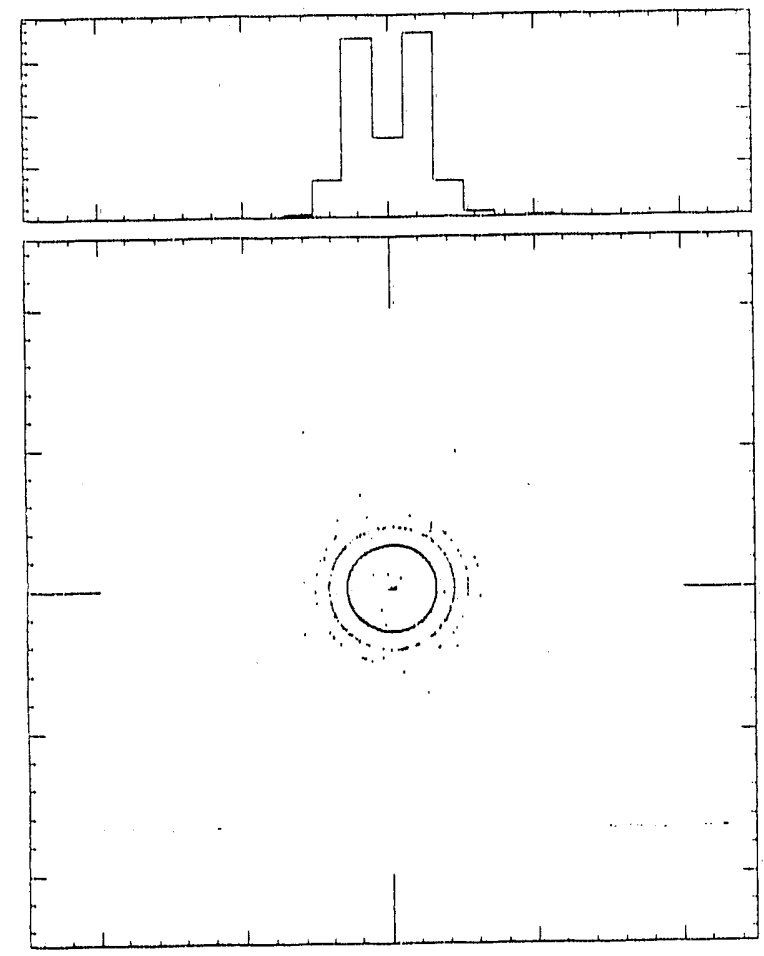

USERMDISK.[XUS.SHADOW]BEGIN.DAT;4
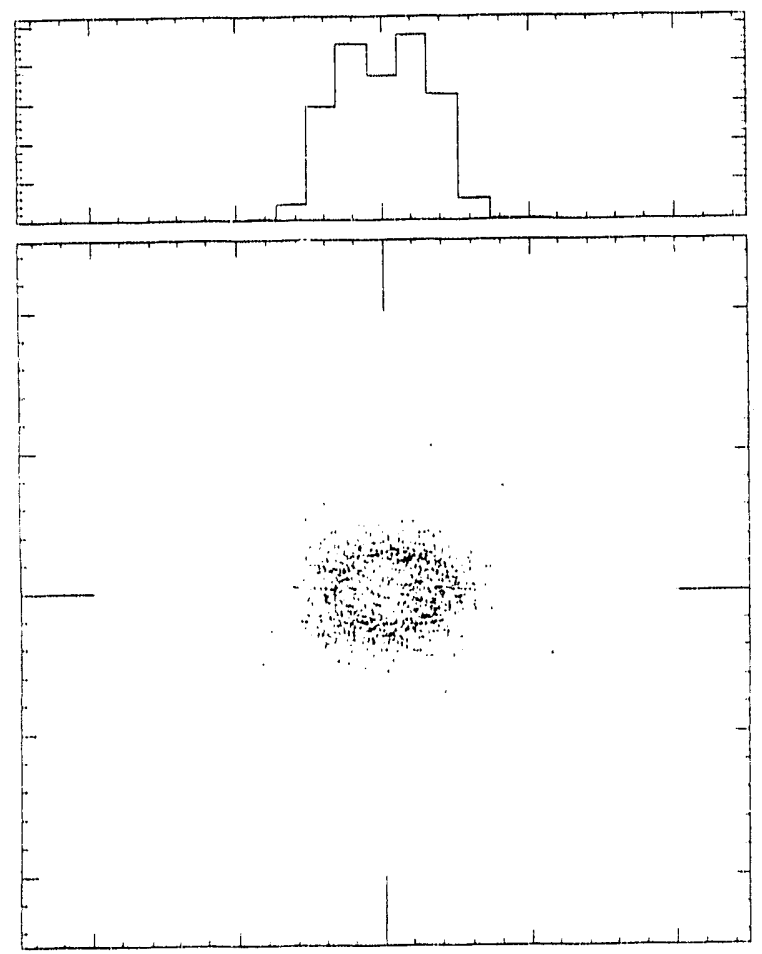

$314,49-199109: 19$

1 is:0 $4=0.95 E=20.07$

$1.0 \%$
$29 \cdot M A R-199109: 24$

$U A: 1 K=0.90 E=20.07$

key

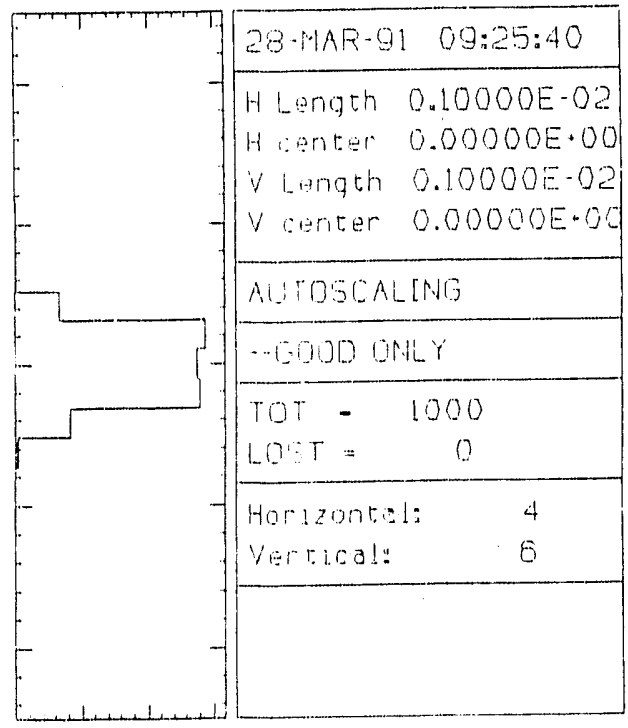

Fig. UA-38. $\mathrm{K}=0.9$, second harmonic. 

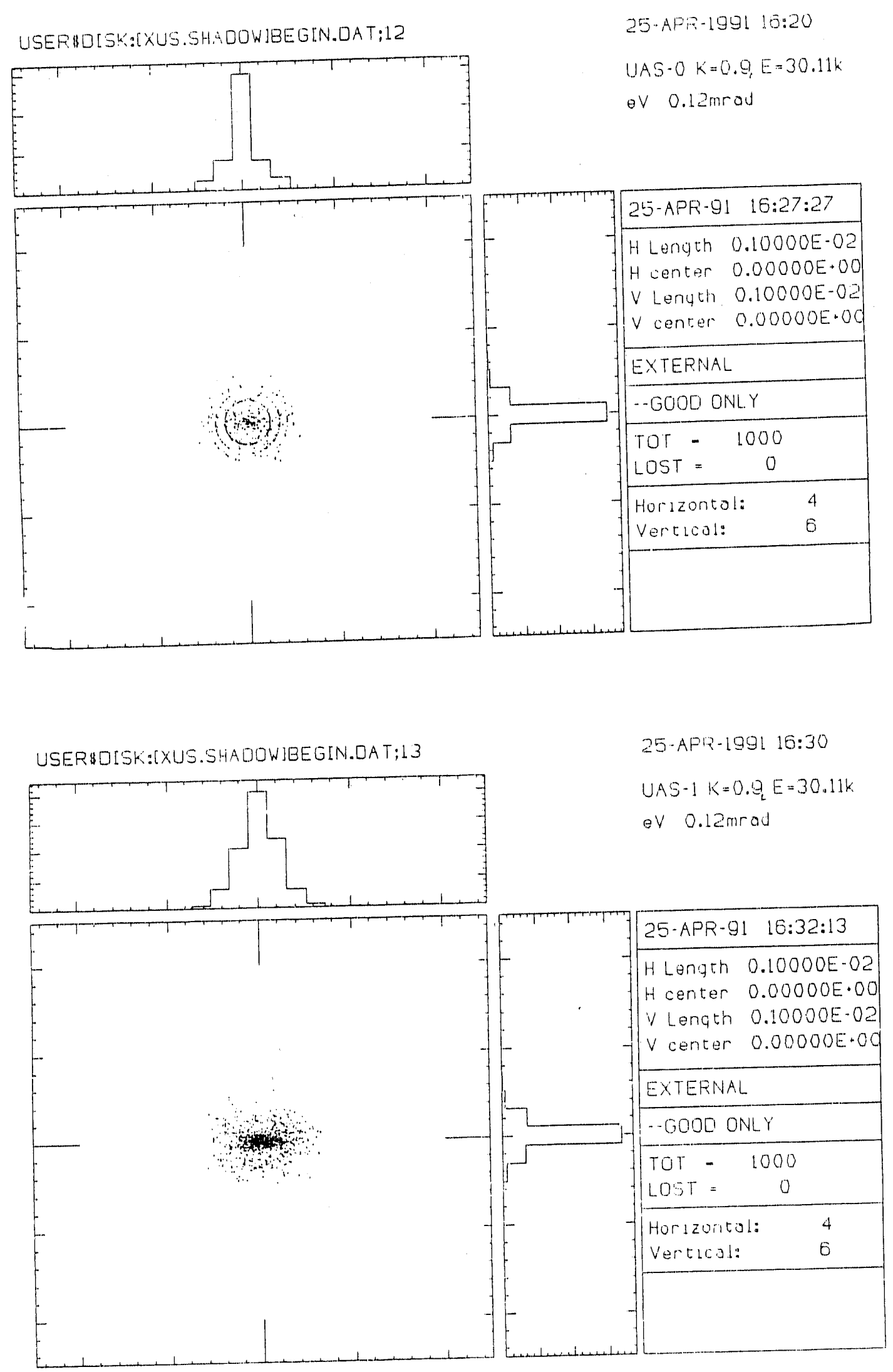

Fig. UA-39. $\mathrm{K}=0.9$, third harmonic. 
USER BDISK:LUS.SHAOOW]BEGIN.DAT:1
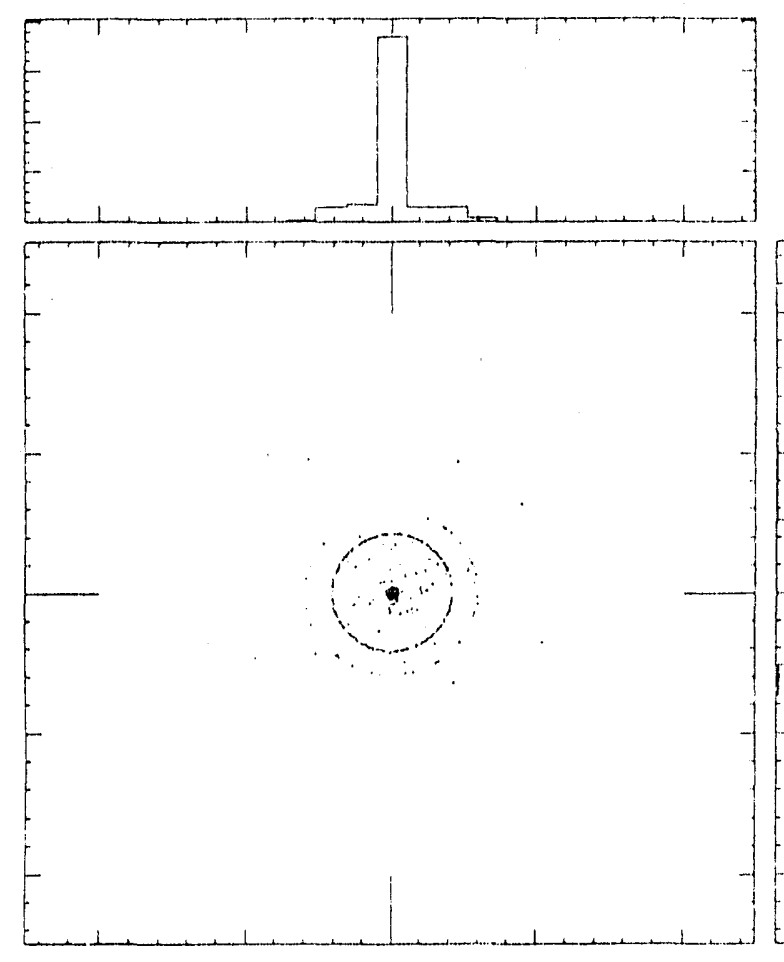

P.MA-19O1 11:18

$1.45-0) 6=0.8+\quad 10.68$

$4.6 \%$
USEPQOLSH:CUS.SHAODWIEEGIN.DAT:2
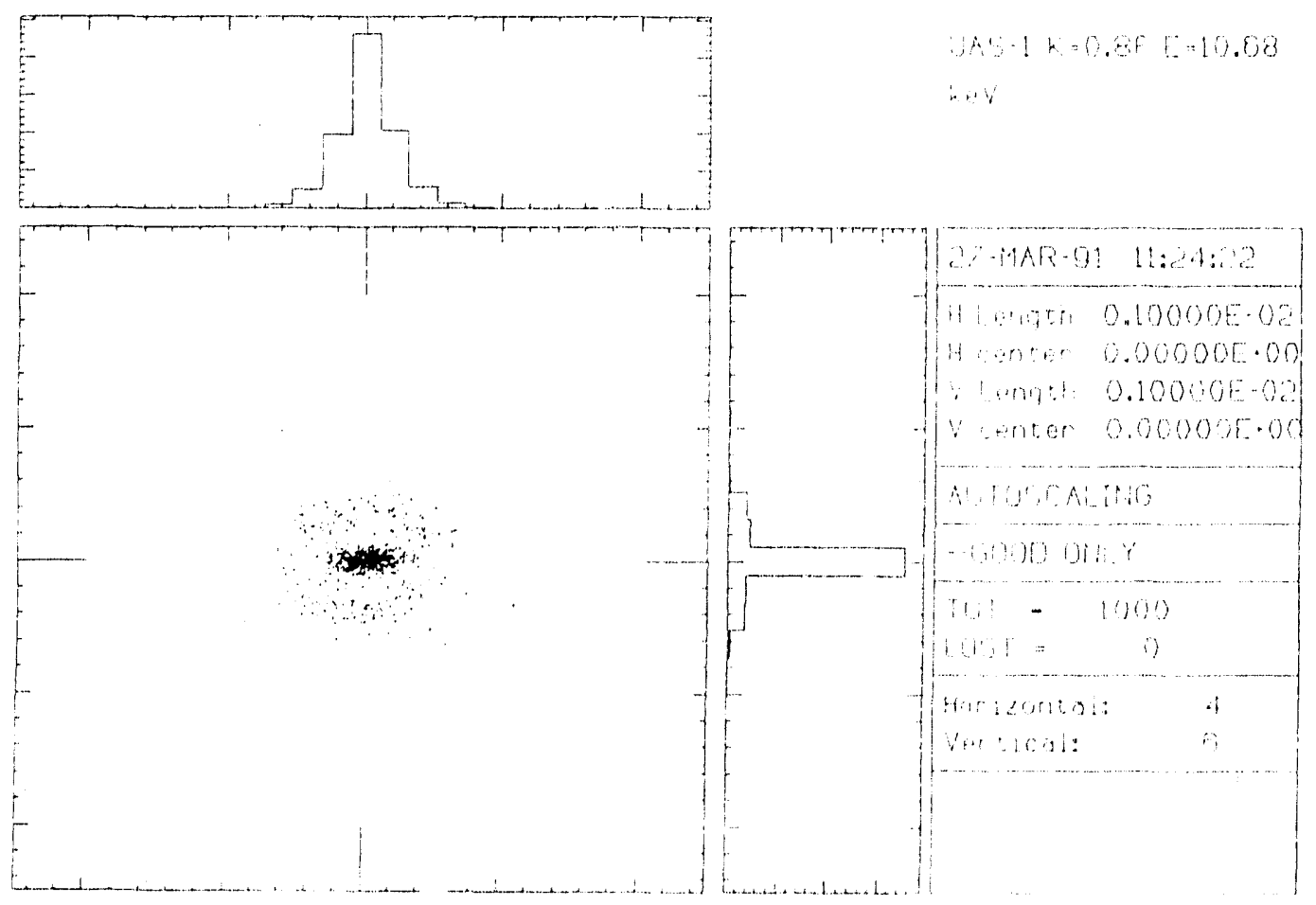

$2+14:-199111.22$

A. $1 h=0.96 \quad 6=10.08$

in.

Fig. UA-40. $\mathrm{K}=0.8$, first harmonic. 

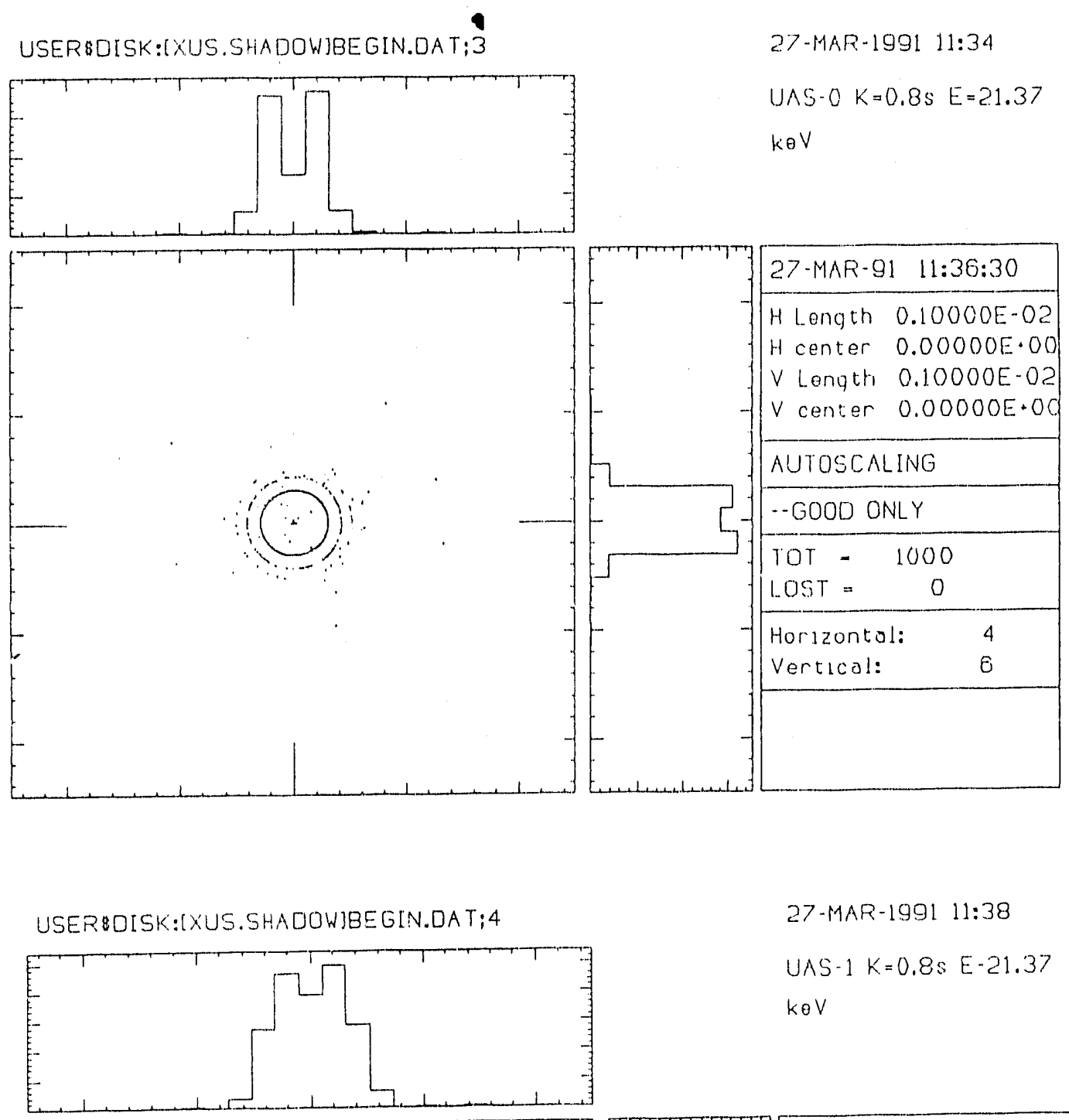

27-MAR-1991 11:38
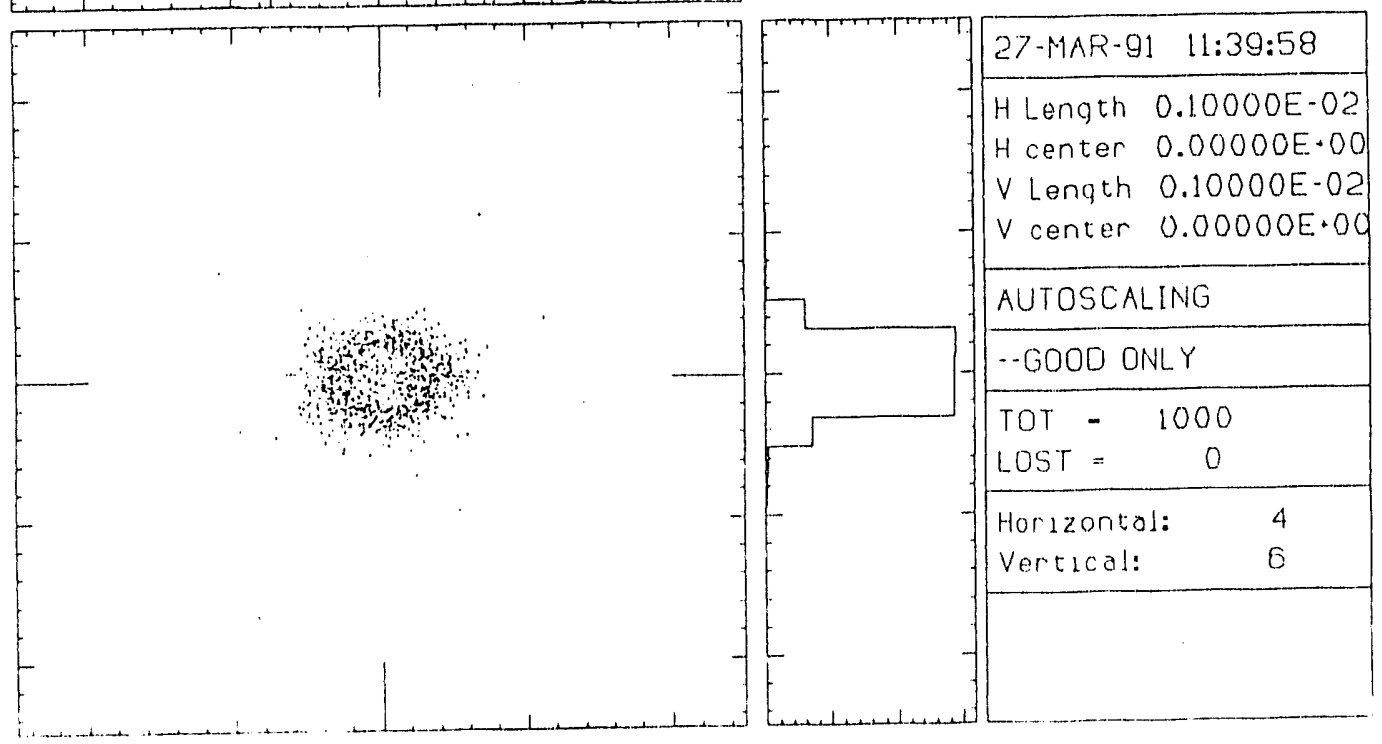

Fig. UA-41. $\mathrm{K}=0.8$, second harmonic. 
USER8DISK:LXS.SHALOWIBEGIN.OA T:15
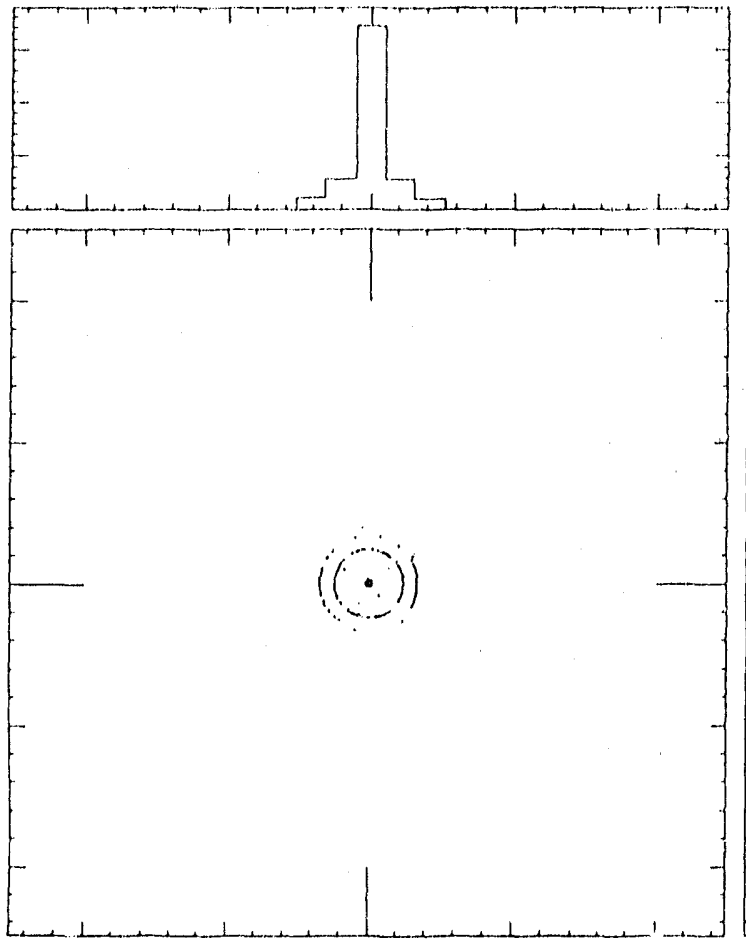

USERBOISK:IXU,.SHAOOWIBEGIN.DAT;IE

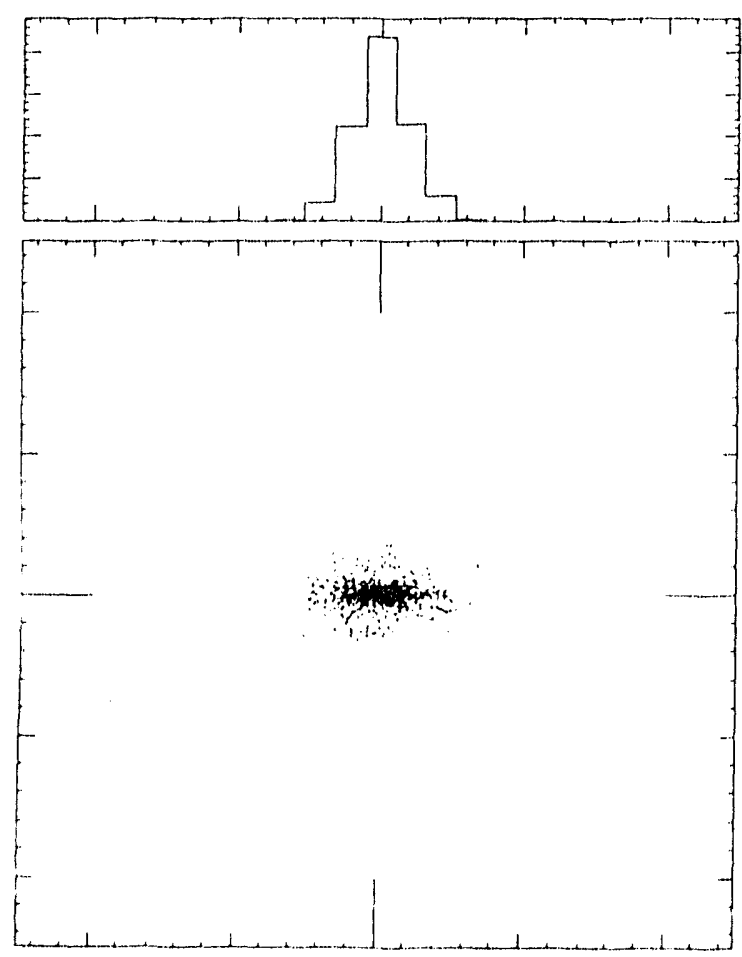

$9 \cdot 4: 1 \cdot 190109: 14$

UAS. () $K=0.8 \% R=32.055^{\circ}$

hov (). () 33 men 011

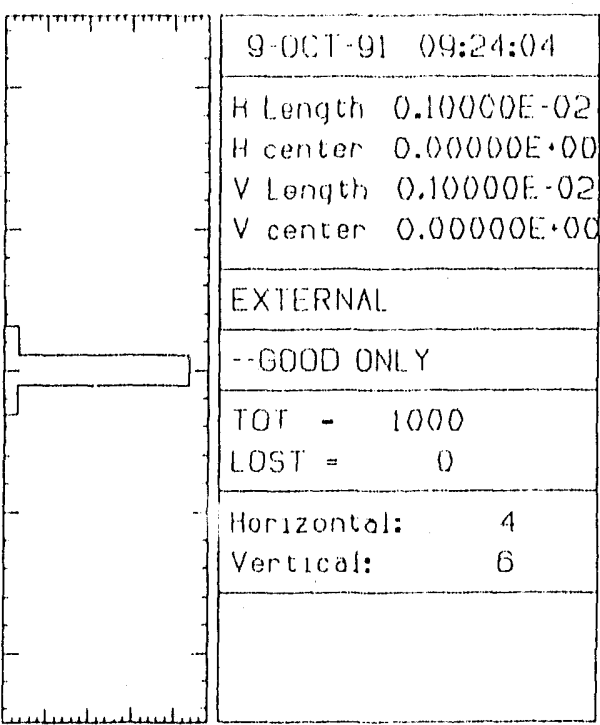

$9-0 T^{2} \cdot 199109: 26$

UAS-1 $K=0.8 E=32.05$

keV O.0umr ad

Fig. UA-42. $\mathrm{K}=0.8$, third harmonic. 
USERBOISK.:XUS.SHAOOWIBEGIN.DAT:I
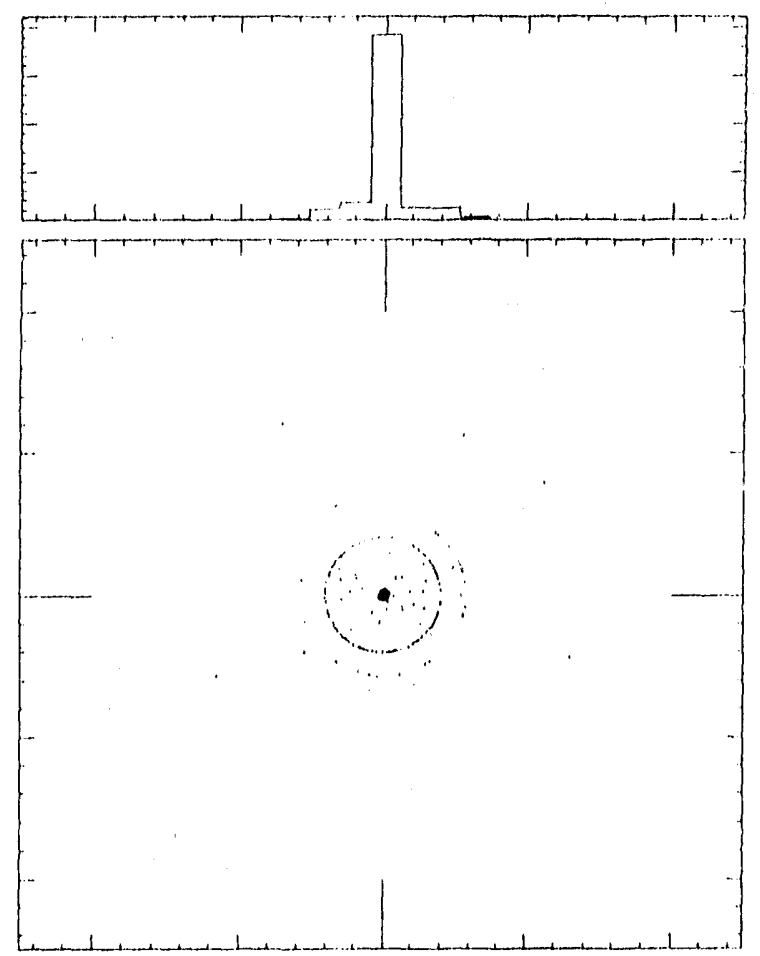

USER.MULSK:IXUS.SHADOWIBEGIN.DATTZ2
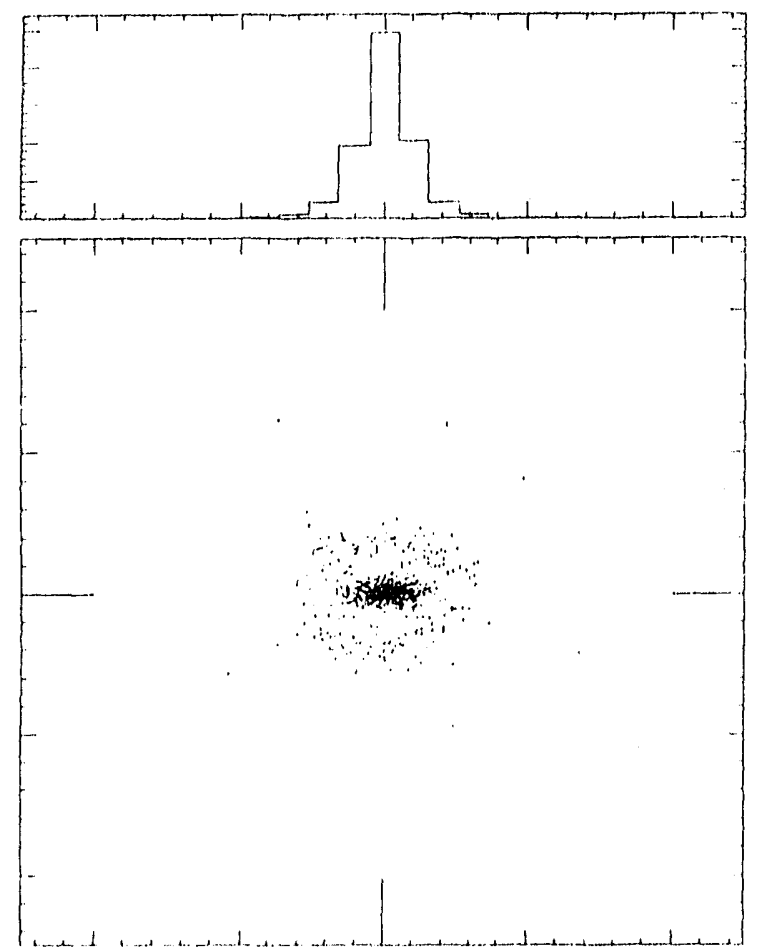

25 -MAK $1991 \quad 15: 13$

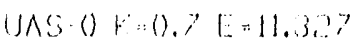

kov

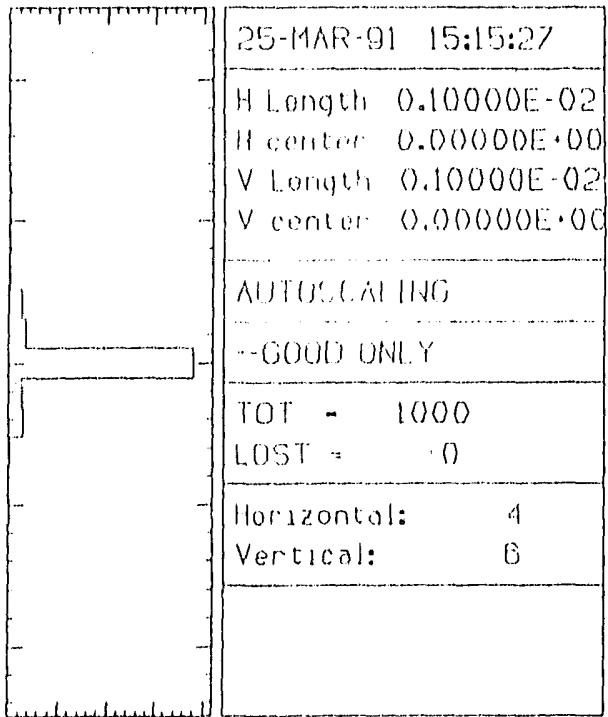

$25-M A R-1991 \quad 15: 17$

UAS-1k.0.7 E=11.327

kov

\begin{tabular}{|c|c|c|}
\hline & $25-M A R-91$ & $15: 19: 11$ \\
\hline & $\left\{\begin{array}{l}H \text { Length } \\
H \text { center } \\
V \text { Lenyth } \\
V \text { center }\end{array}\right.$ & $\begin{array}{l}0.10000 E-02 \\
0.00000 E \cdot 00 \\
0.10000 E-02 \\
0.00000 E \cdot 00\end{array}$ \\
\hline & AUTOSCALL & ING3 \\
\hline & - GOOJD OND & \\
\hline & $\begin{array}{l}\text { TOT }-1 \\
\text { LOST }=\end{array}$ & $\begin{array}{c}1000 \\
0\end{array}$ \\
\hline & $\begin{array}{l}\text { Hortzontal: } \\
\text { Vertical: }\end{array}$ & $\begin{array}{l}\quad 4 \\
\quad 6\end{array}$ \\
\hline & & \\
\hline
\end{tabular}

Fig. UA-43. $\quad \mathrm{K}=0.7$, first harmonic. 
USERBDISK:IXUS.SHADOWIBEGIN.BAT:3
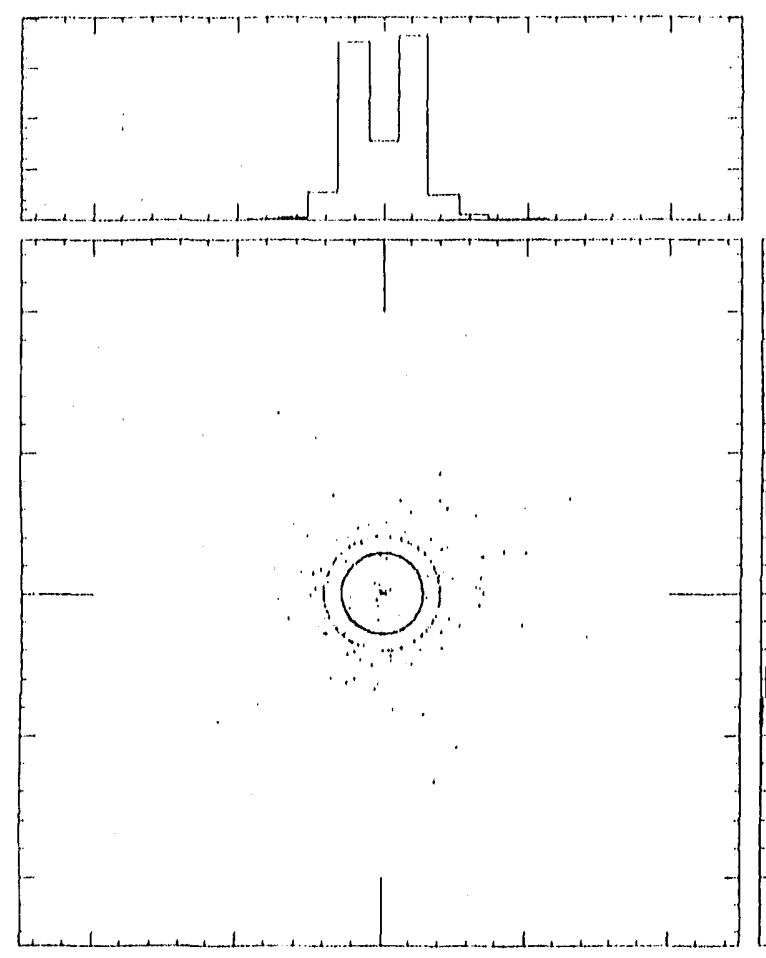

$2 ! 3-N A R \cdot 195115933$

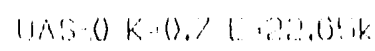

oV

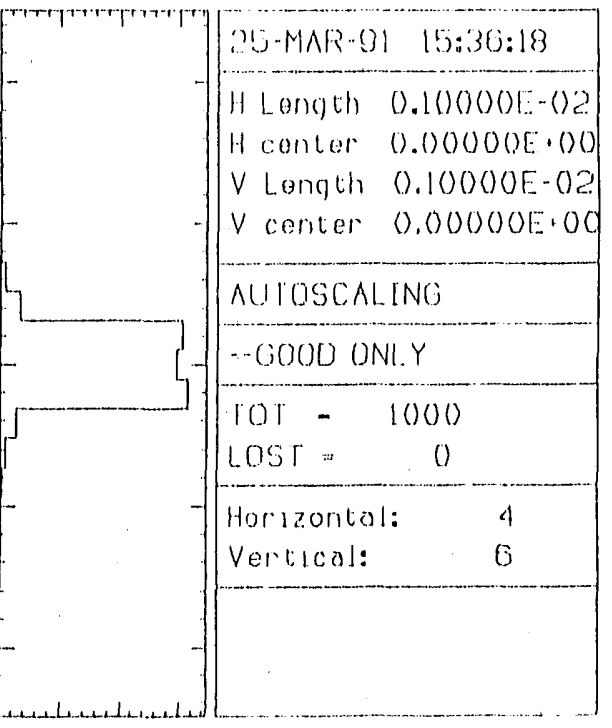

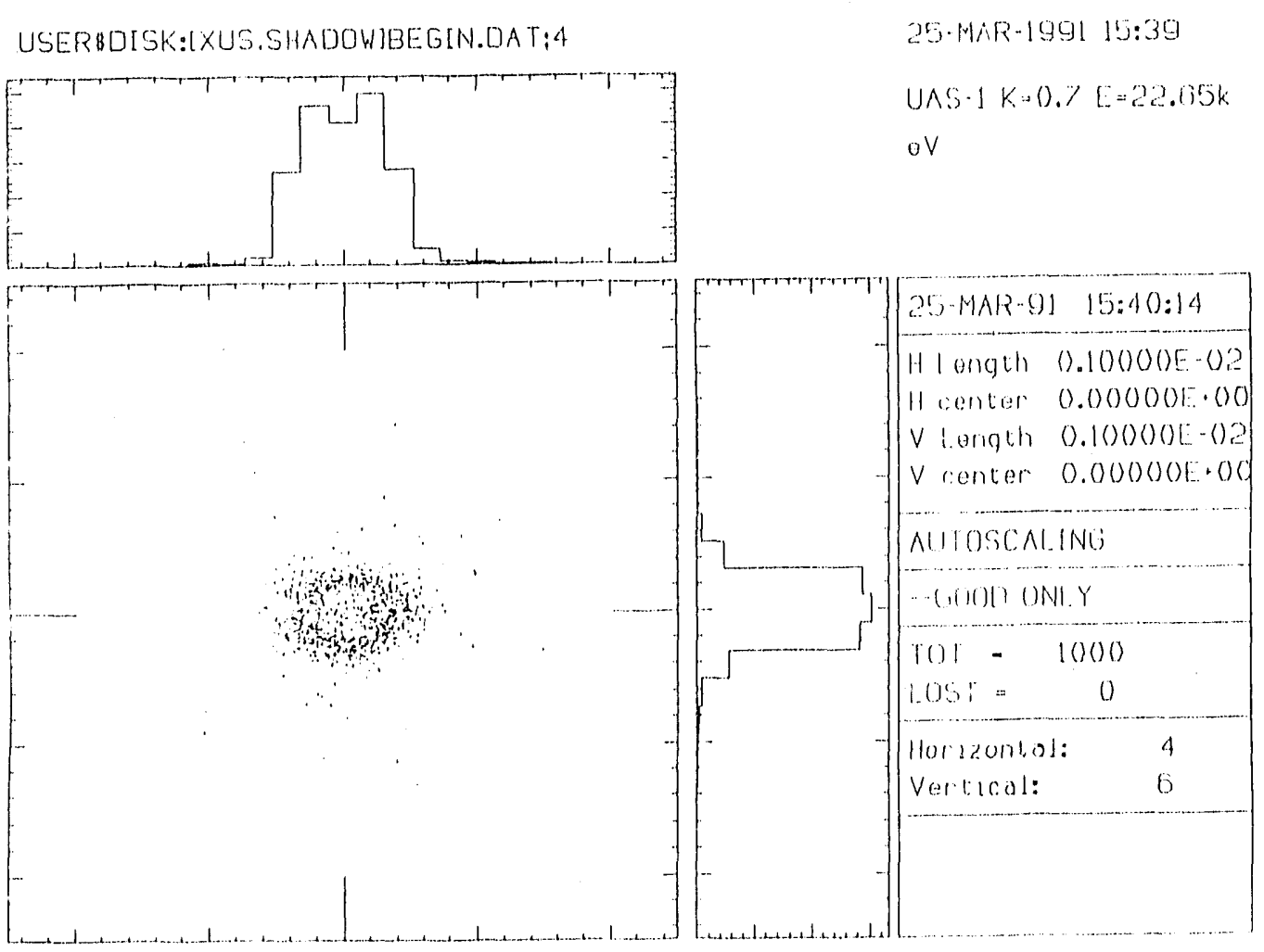

Fig. UA-44. $K=0.7$, second harmonic. 

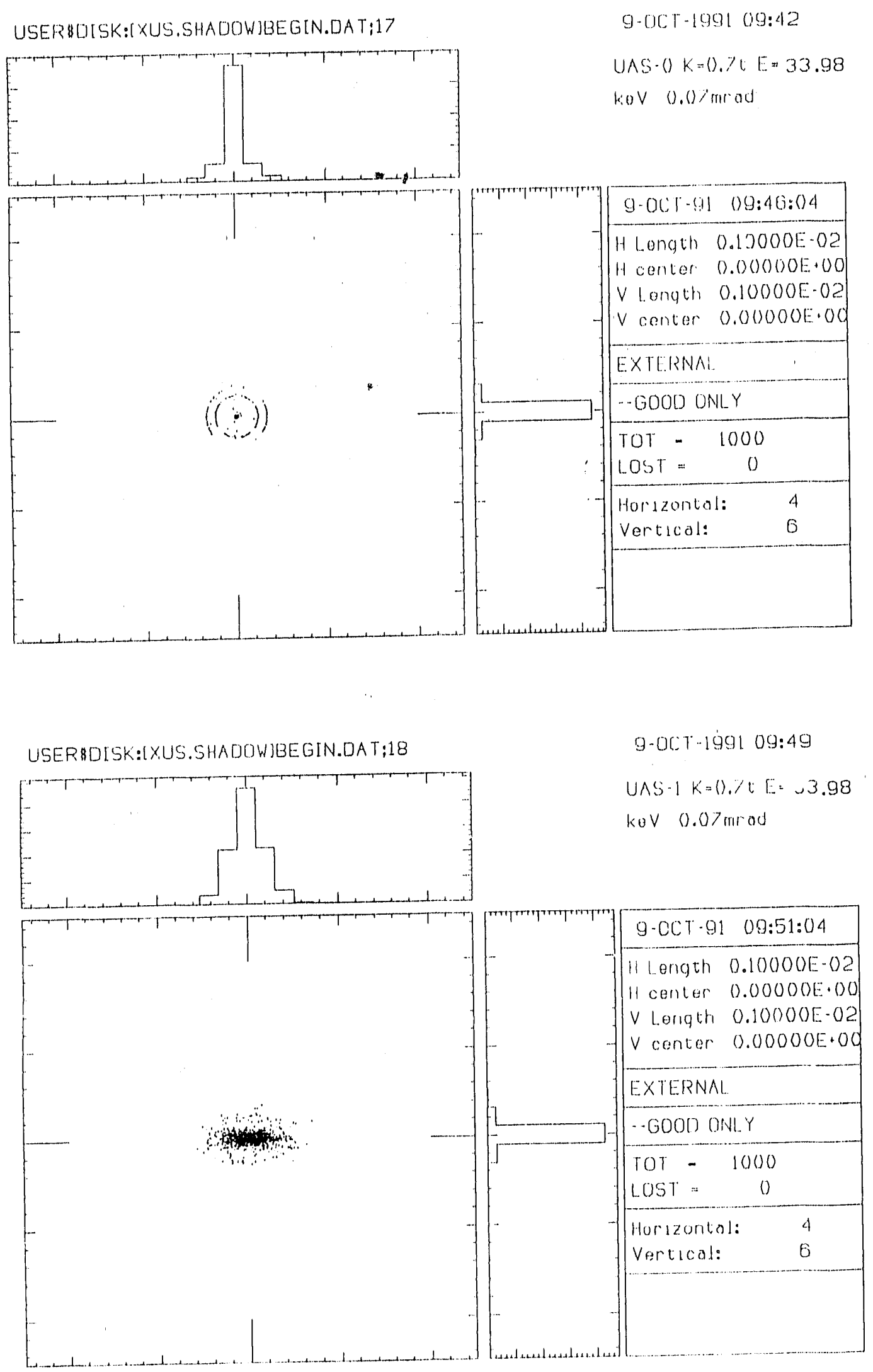

Fig. UA-45. $\quad \mathrm{K}=0.7$, third harmonic. 
USEF\&OISHIELAI.IFACY.TESIIBEGIN.TAN:A3
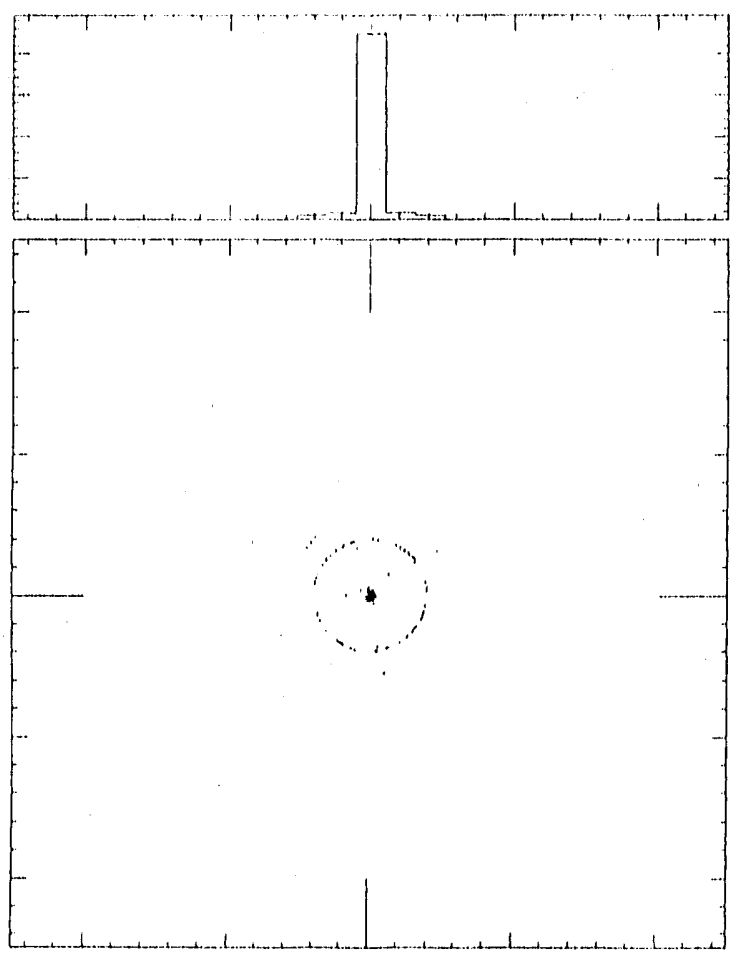

USERBOISKIOLAI.TRACY.TESTIBEGIN.OIAT:44

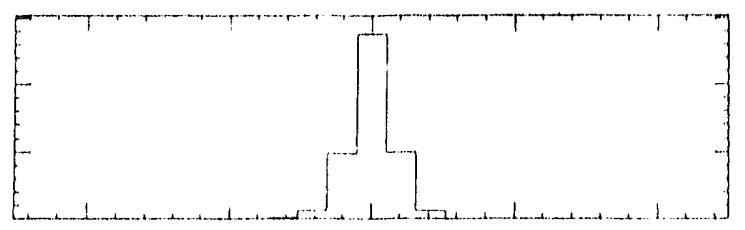

$\cong$

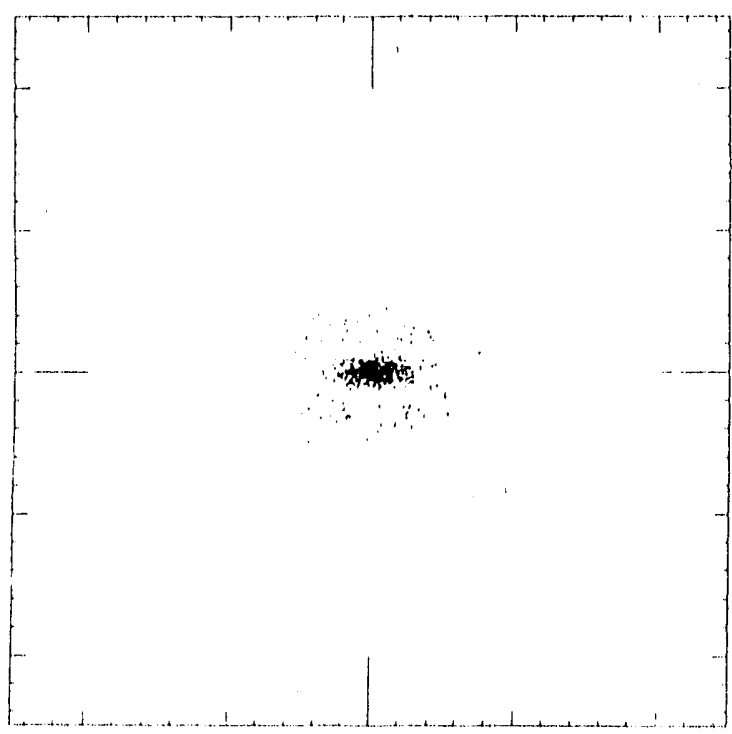

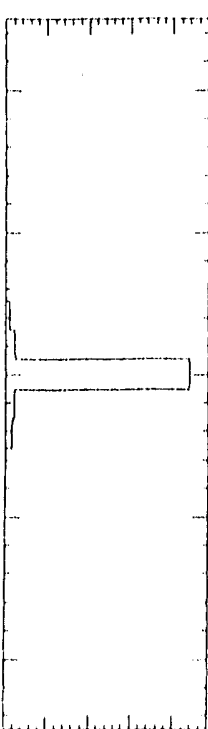

1․ $120190222: 45$

$1.49 \cdot 0 k=0.6 \% 11.95 k$

$\because \vee$

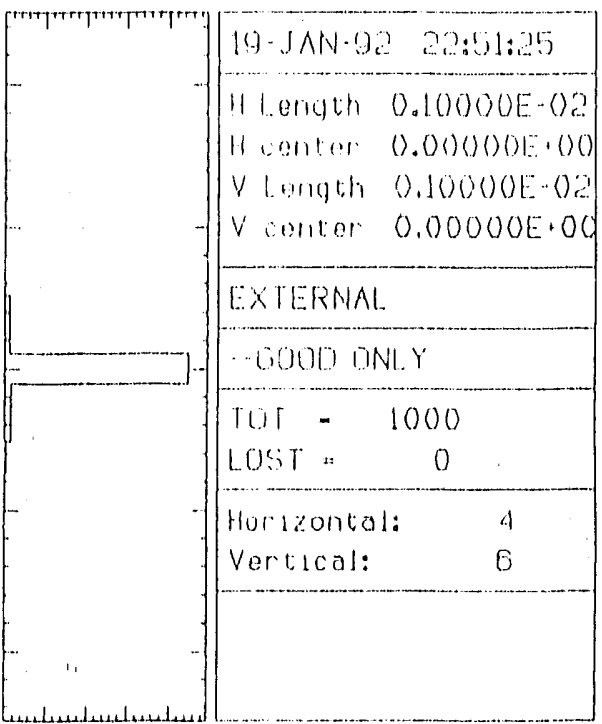

19. $\operatorname{lnN} 190220152$

$13 A .16=0.0 \mathrm{E}=11.96 \mathrm{k}$

ov

\begin{tabular}{|c|c|}
\hline $19 \cdot \operatorname{IAN}-92 ?$ & $22: 54: 46$ \\
\hline$H$ Languth 0 & $0.10000 E \cdot 02$ \\
\hline 11 comber 0 & $0.60000 E \cdot 00$ \\
\hline V longth o & $0.10000 E-02$ \\
\hline V concer & $0.00000 E \cdot 00$ \\
\hline EXIIRNAL & \\
\hline GluO GN & \\
\hline $\mid \begin{array}{lll}10 \Gamma-1 & -1 \\
1.051 & =\end{array}$ & $\begin{array}{c}000 \\
0\end{array}$ \\
\hline Holdoutal: onta & 4 \\
\hline Verturol: & $\theta$ \\
\hline
\end{tabular}

Fig. UA-46. $\mathrm{K}=0.6$, first harmonic. 
USERHOISK:CXUS.SHADOWIBEGIN.DATI19
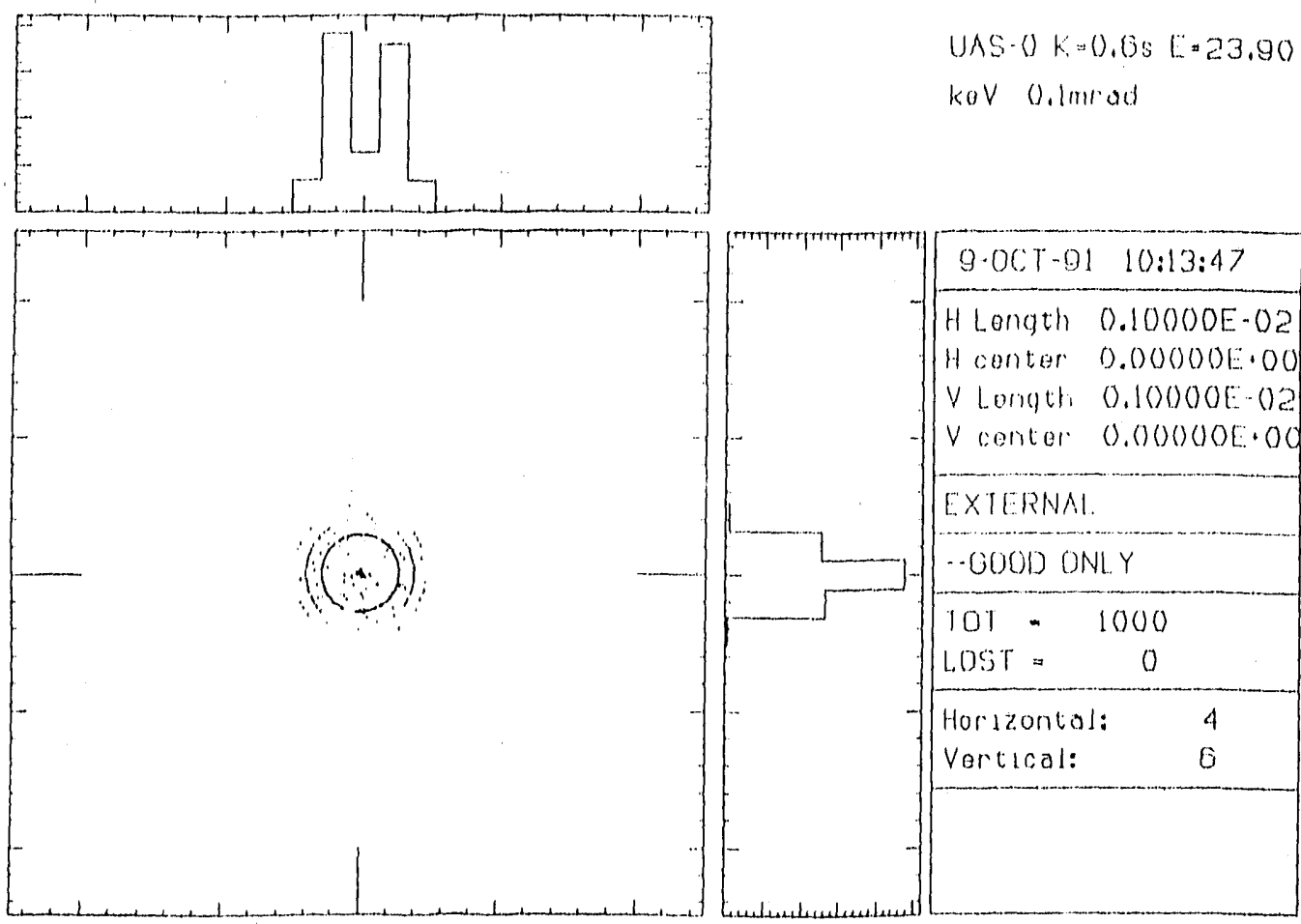

LSEFBDISK:LXUS.SHA.DOWIBEGIN.DAT:20
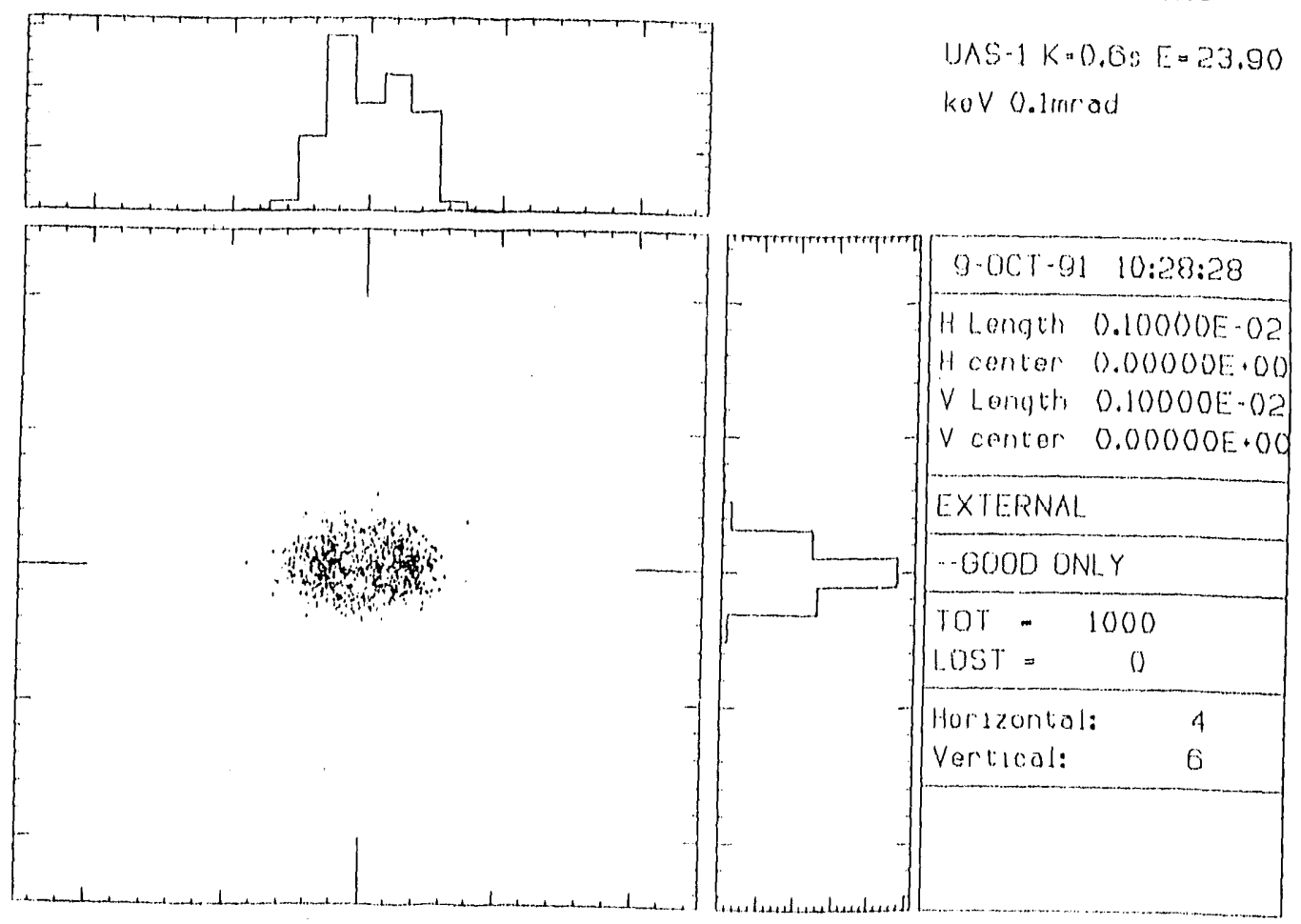

UAS- $K)=(0.038=23.90$

kov $0.1 \mathrm{mrad}$ 


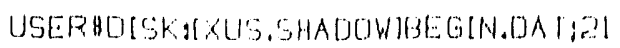
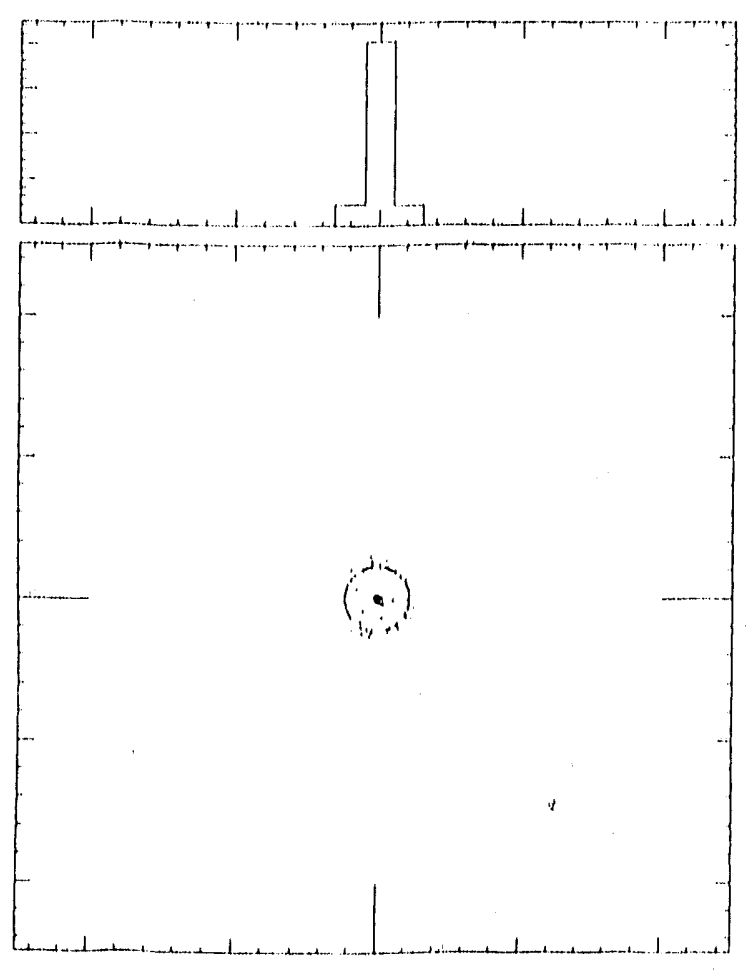

USERBDISK:(X1,3.SHACOWWEEGIN.DA TIA:
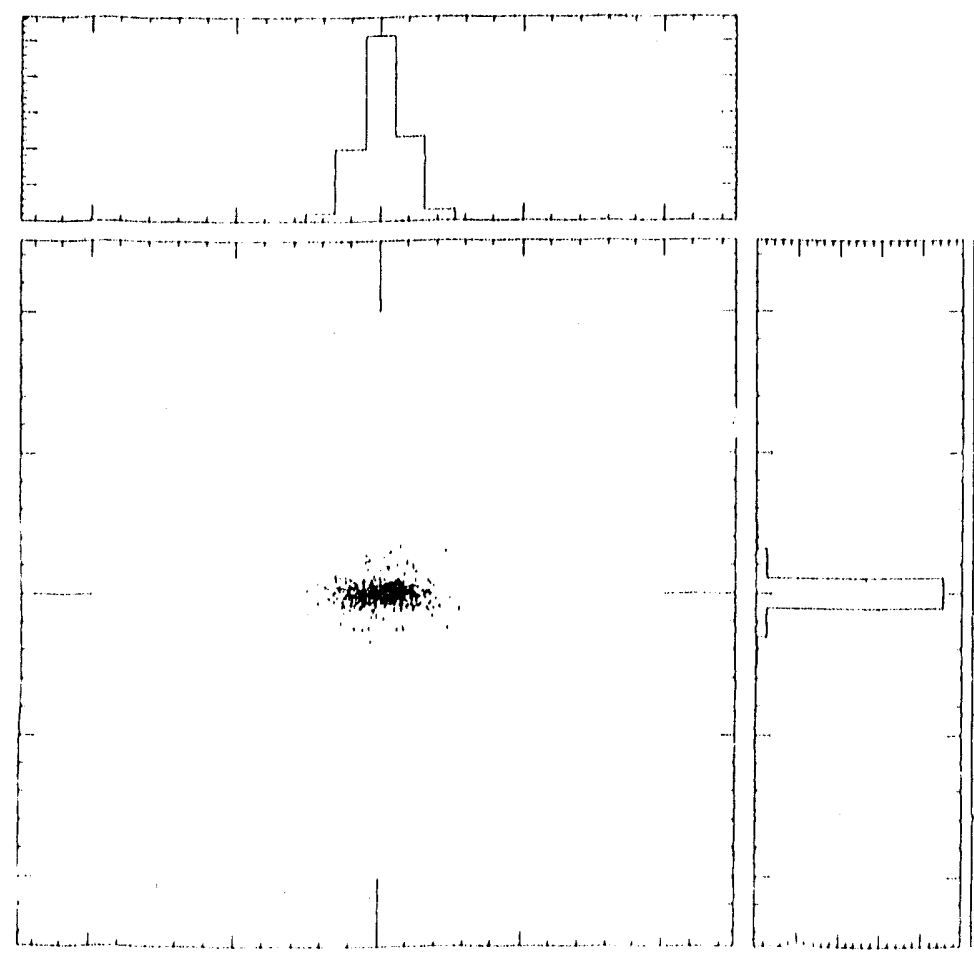

4) 13: T-1981 10:4?

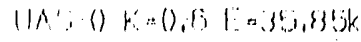

..V (), () bom ad

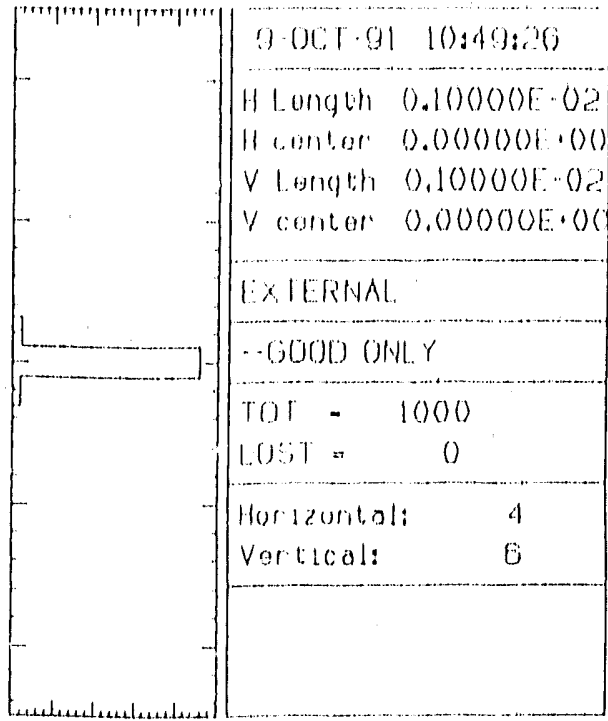

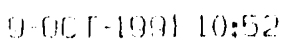

UAG. $1+0.601=30.85$

$k ! y \quad 0,0 b m a d$

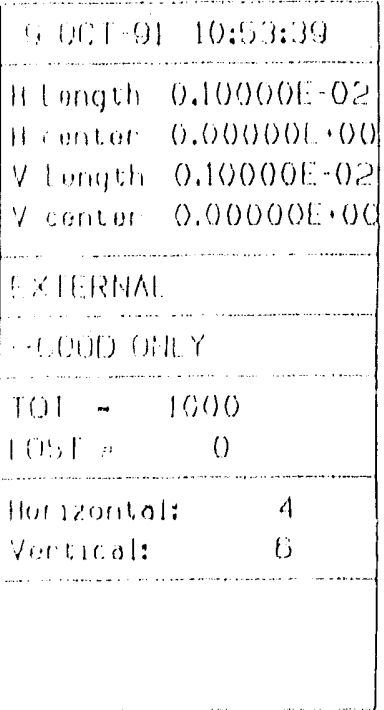

Fig. UA-48, $K=0.6$, third harmonic. 
ISGEAOISK,ABLALITHCY.TESTIBEGIN.OATI3E
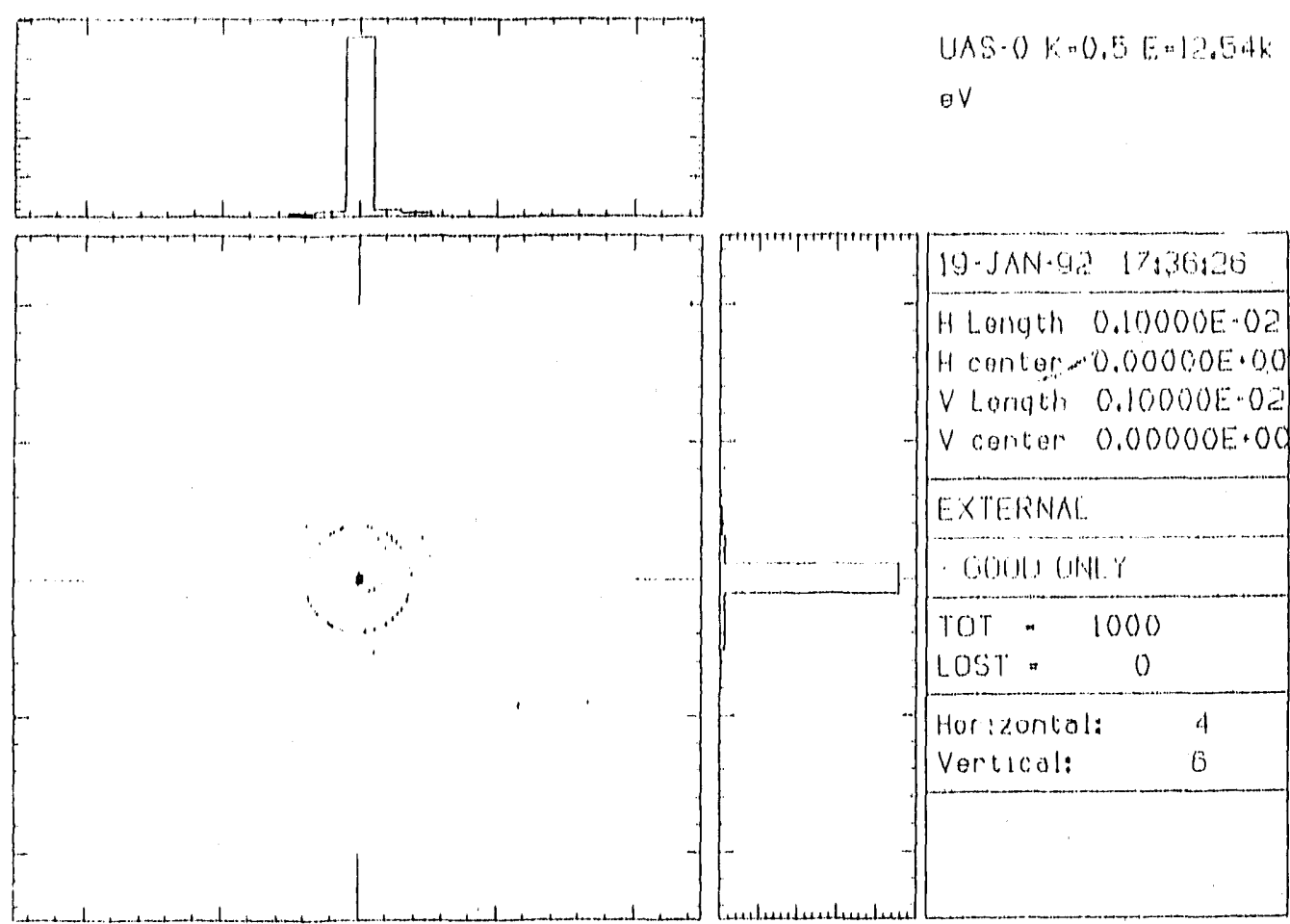

WSERBLISKIBLAI,TRACY.TESTIBEGIN.DAT,37
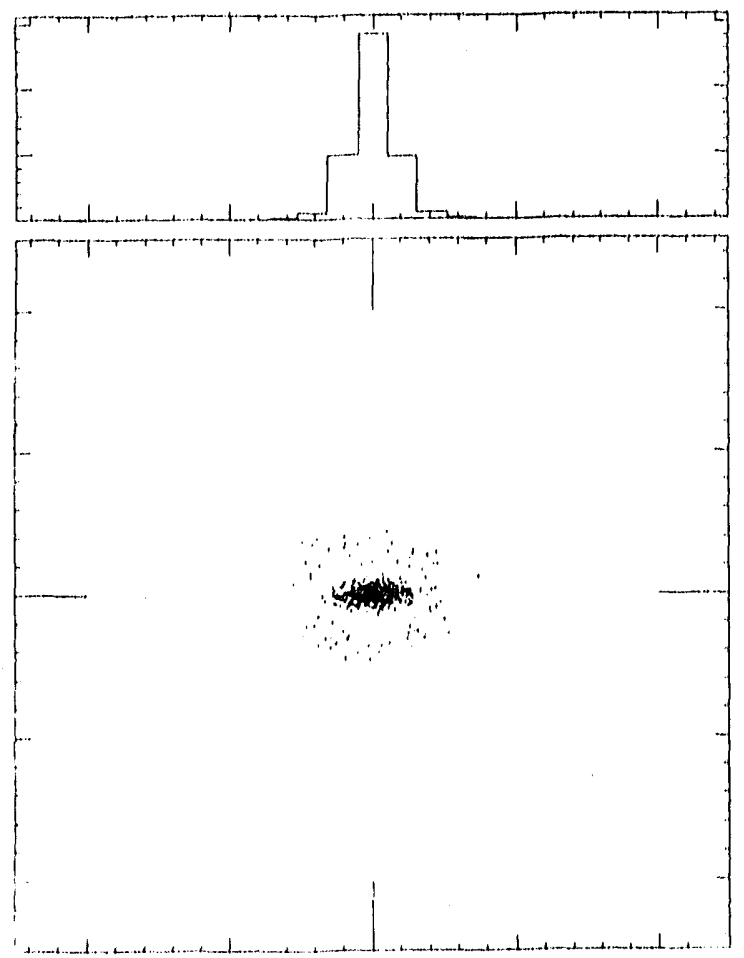

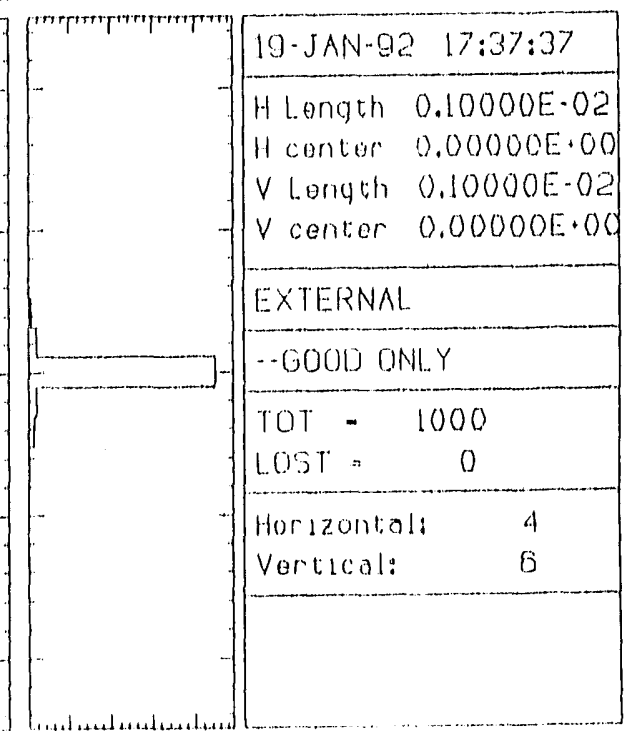

Fig. UA-49. $\quad \mathrm{K}=0.5$, first harmonic. 

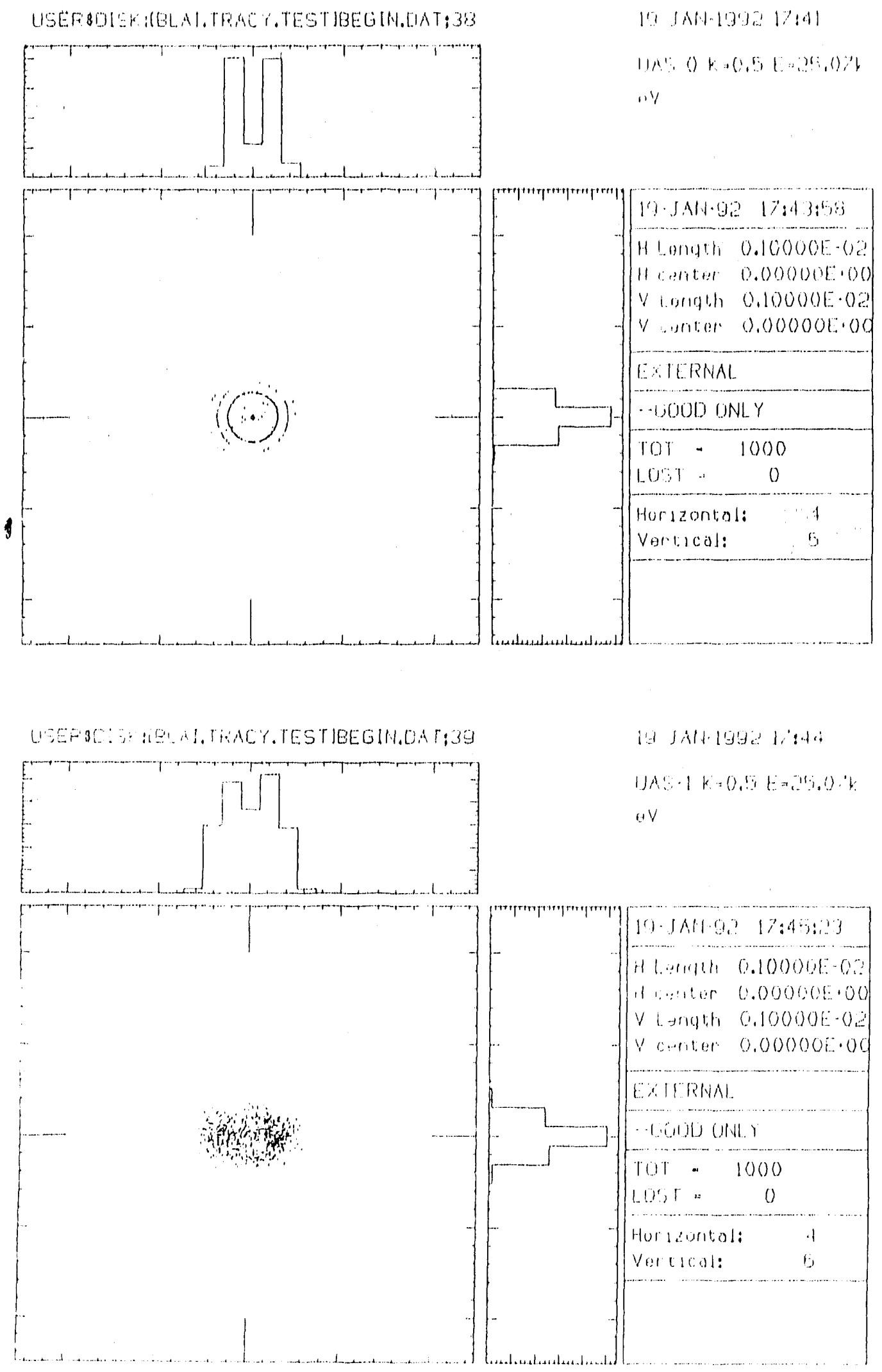

Fig. UA-50. $\quad \mathrm{K}=0.5$, second harmonic. 


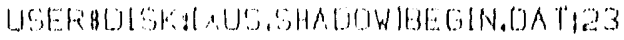
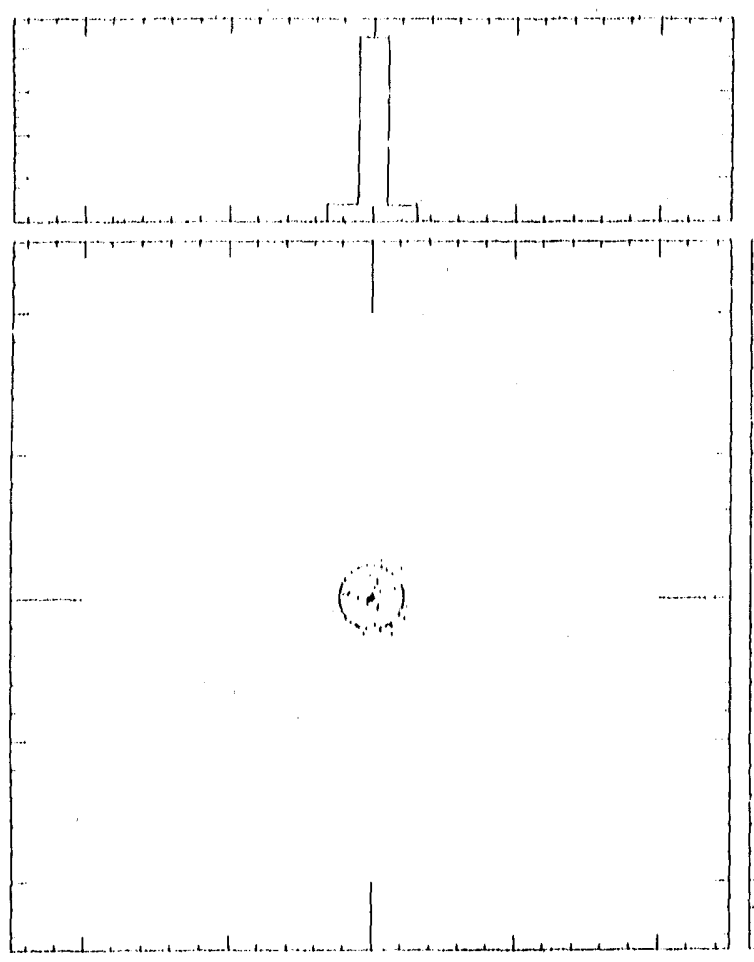

USERBLISKaIXUS.SHALOUWBEGIN.DATIRS
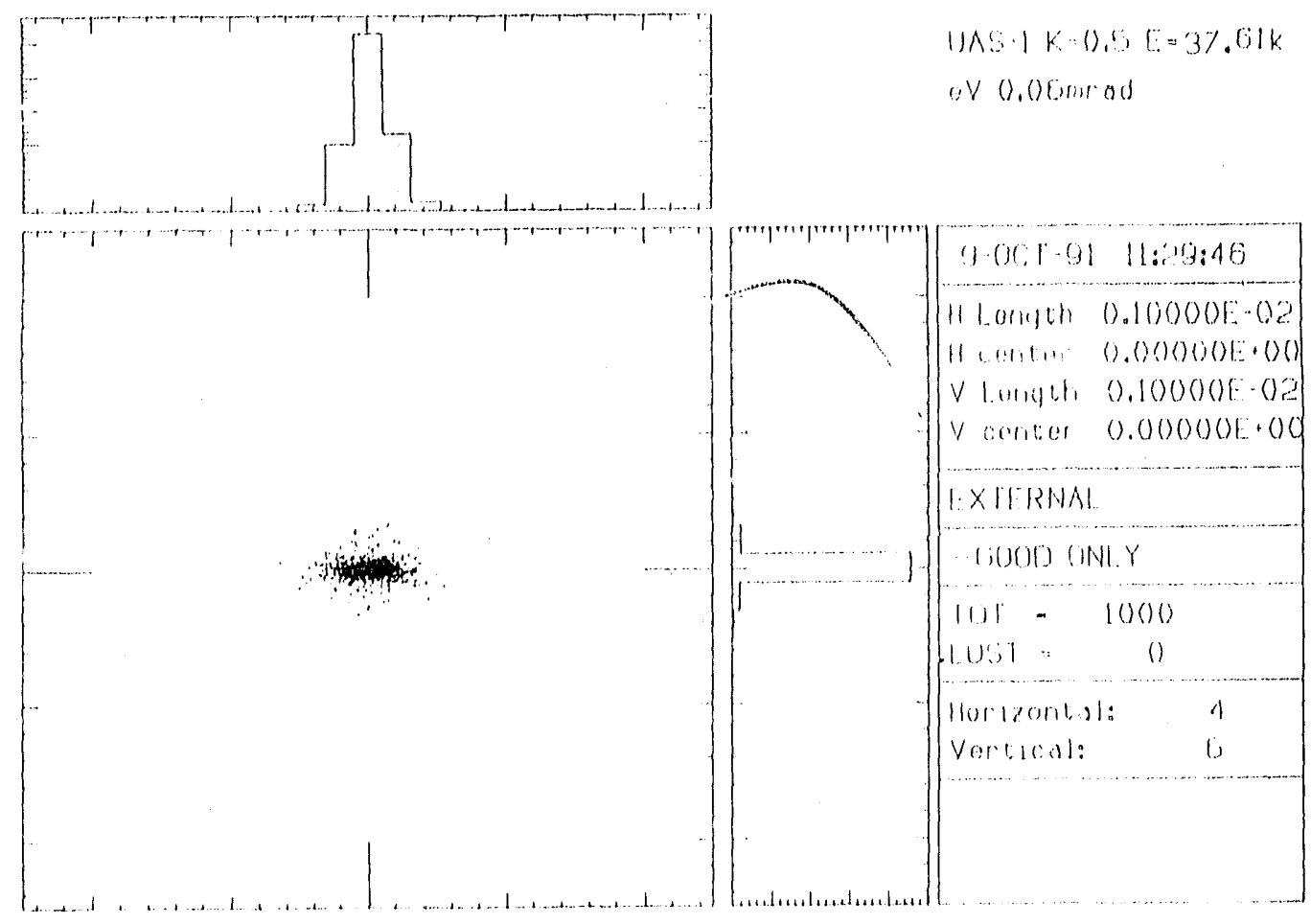

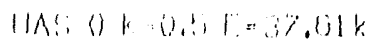

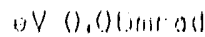

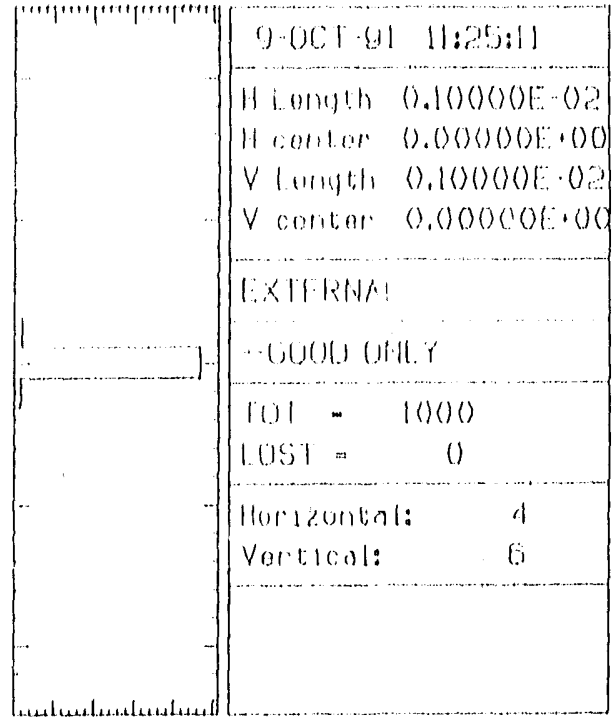

Fig. UA-51. $K=0.5$, third harmonic. 

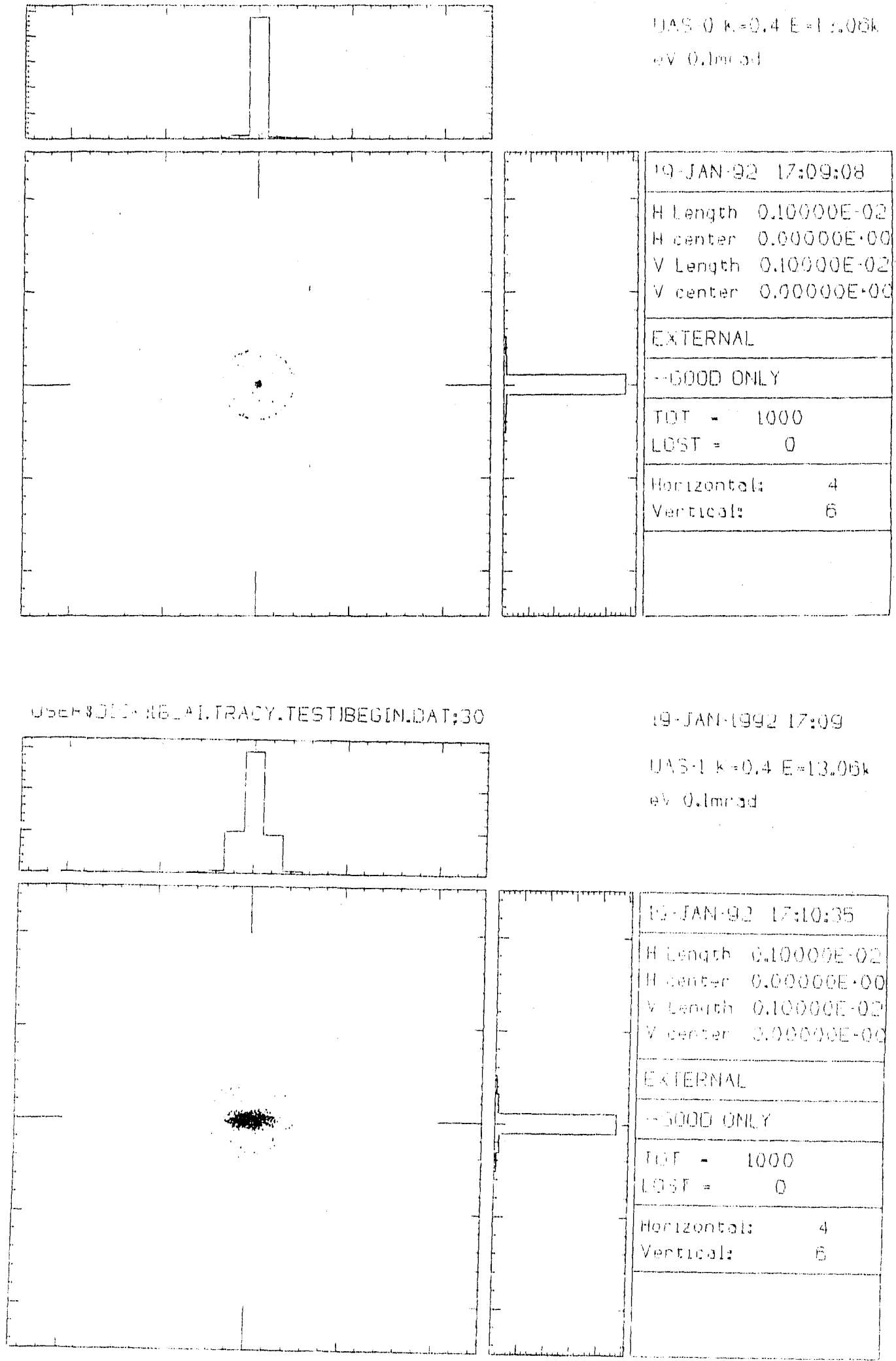

Fig. UA-52. $\mathrm{K}=0.4$, first harmonic. 

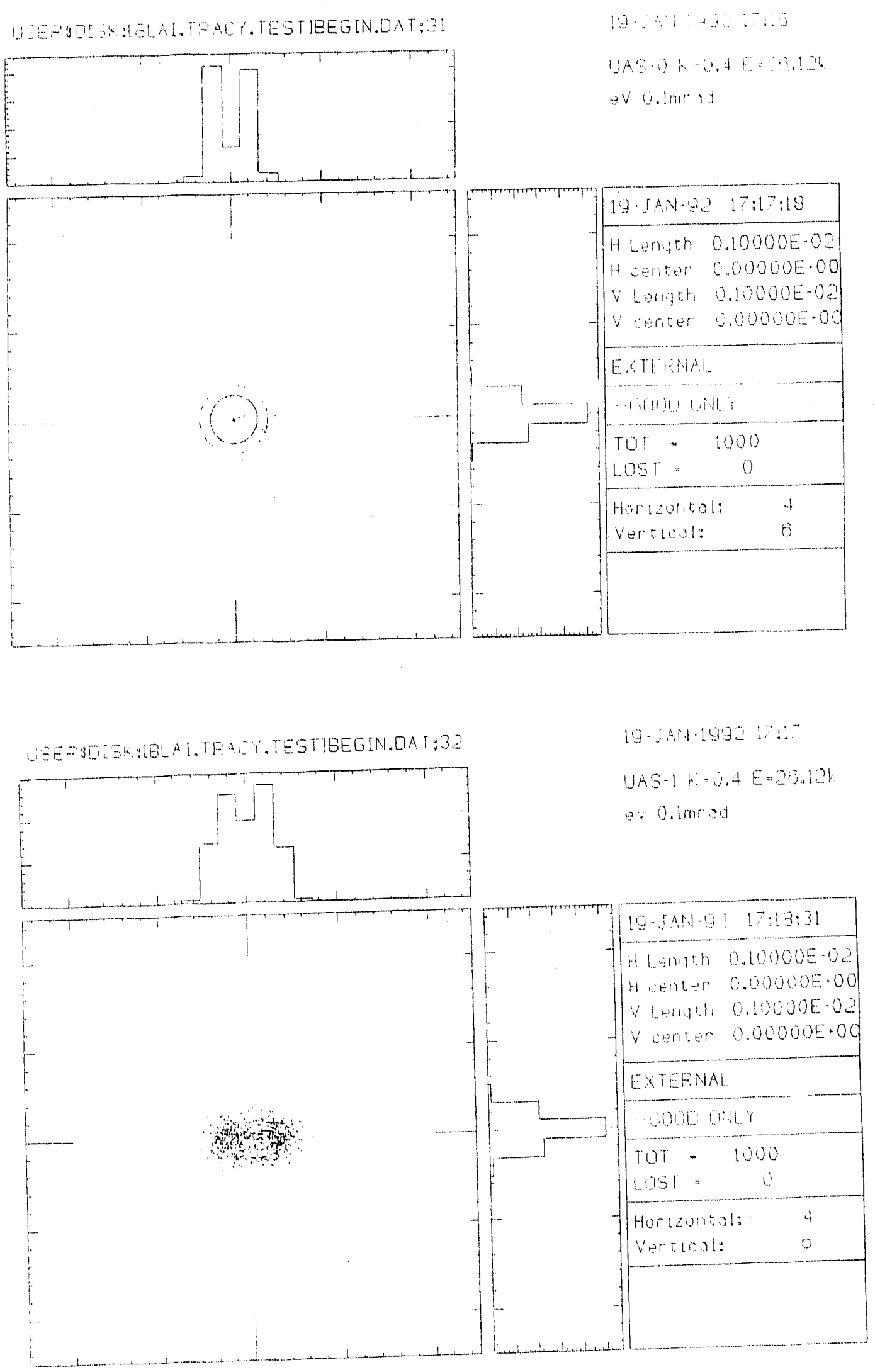

Fig. UA-53. $\quad K=0.4$, second harmonic. 


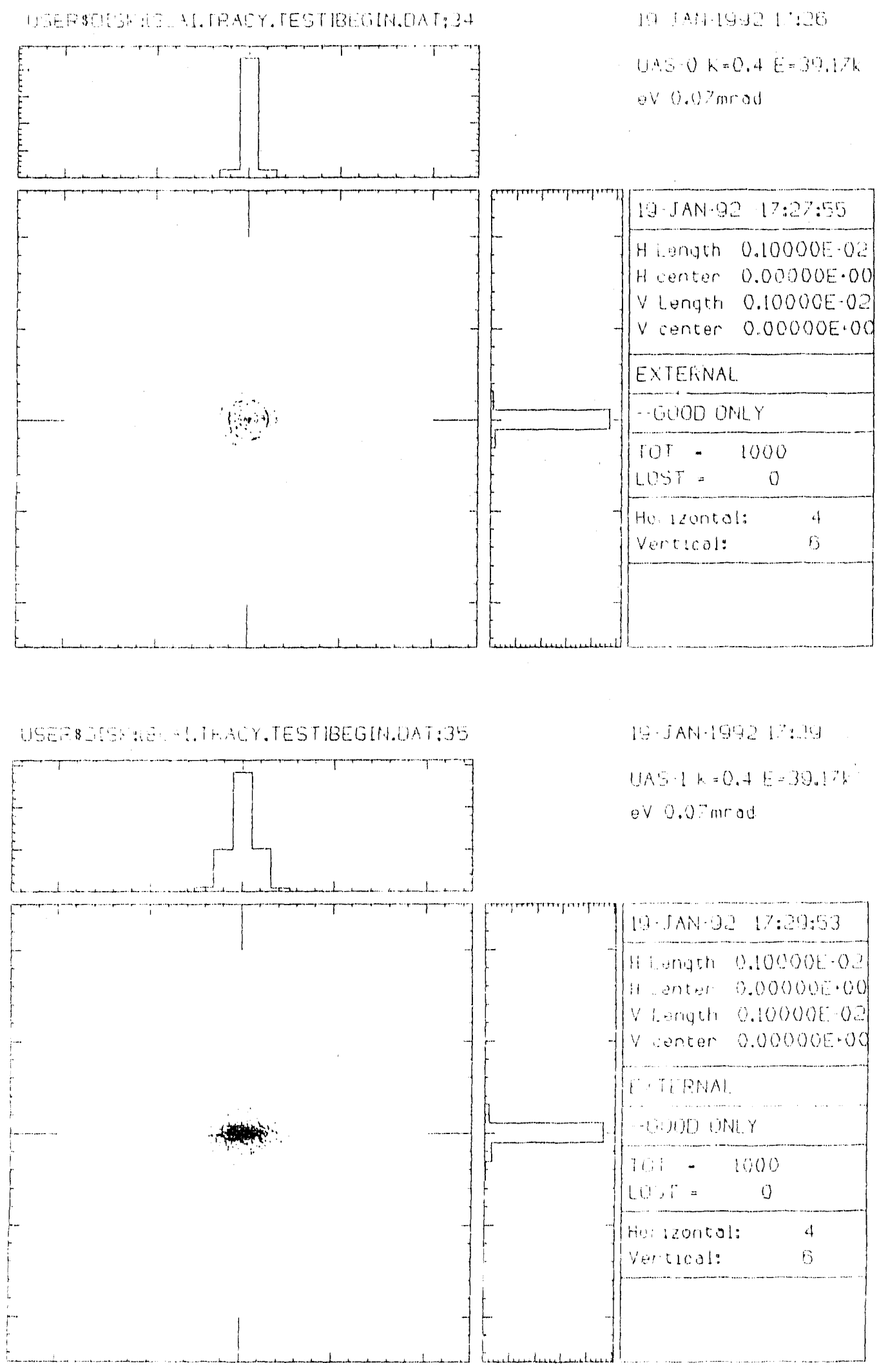

Fig. UA-54. $K=0.4$, third harmonic. 


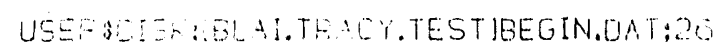
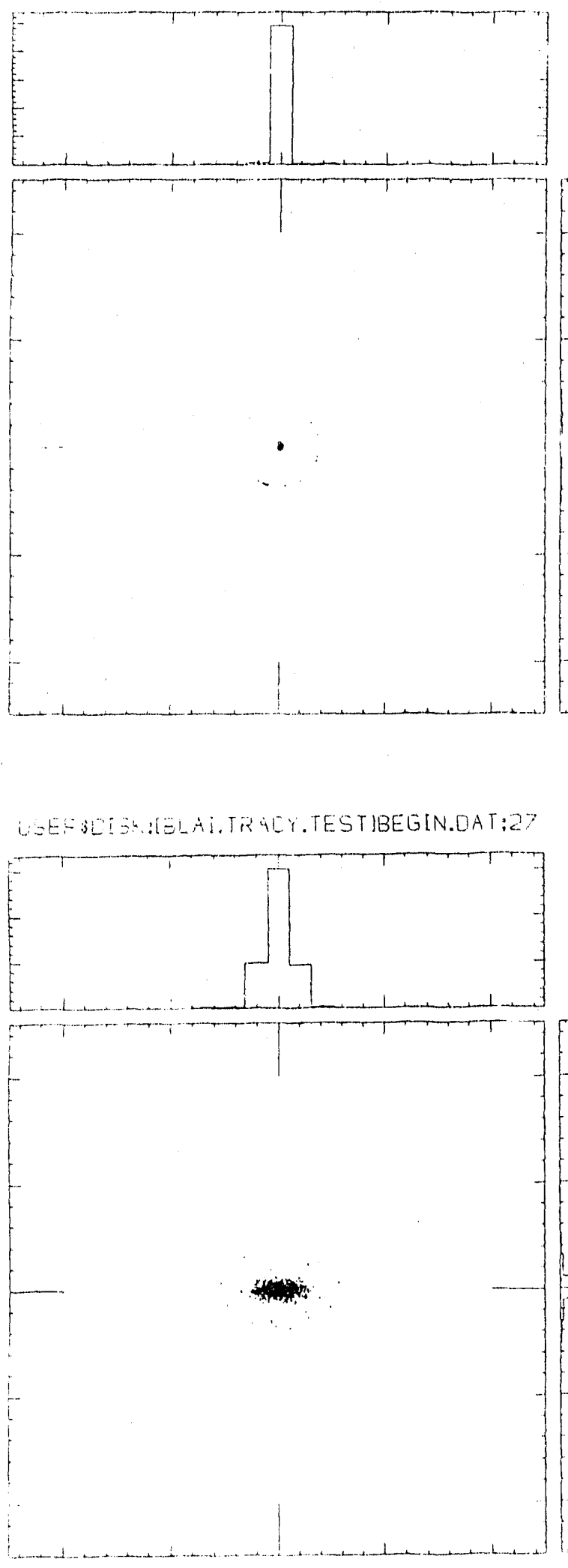

$19 \cdot 3, \cdots \cdot 90,26954$

$A=0)+28: 290 \%$

$\because 40.03000$

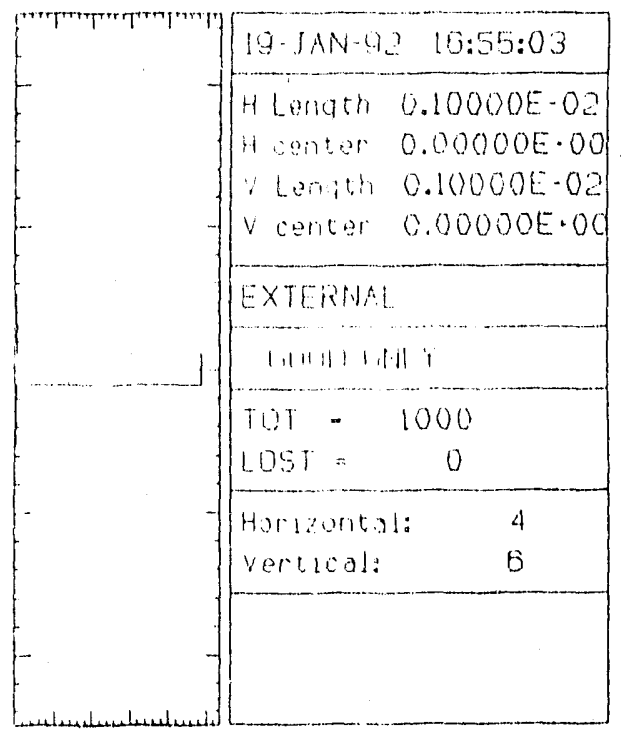

$19-54139216: 55$

UAS $1 K=0.3 E=13.50 k$

eV $0.08 \mathrm{mrod}$

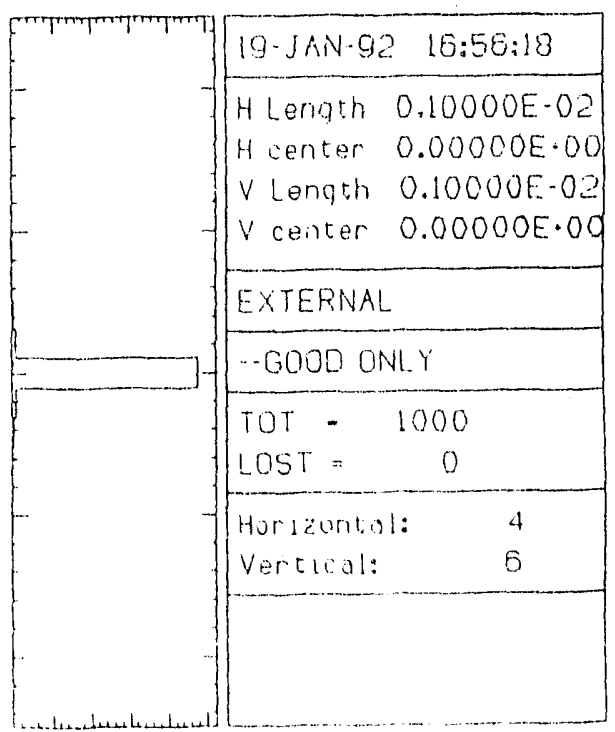

Fig. UA-55. $\quad \mathrm{K}=0.3$, first harmonic. 


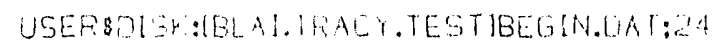
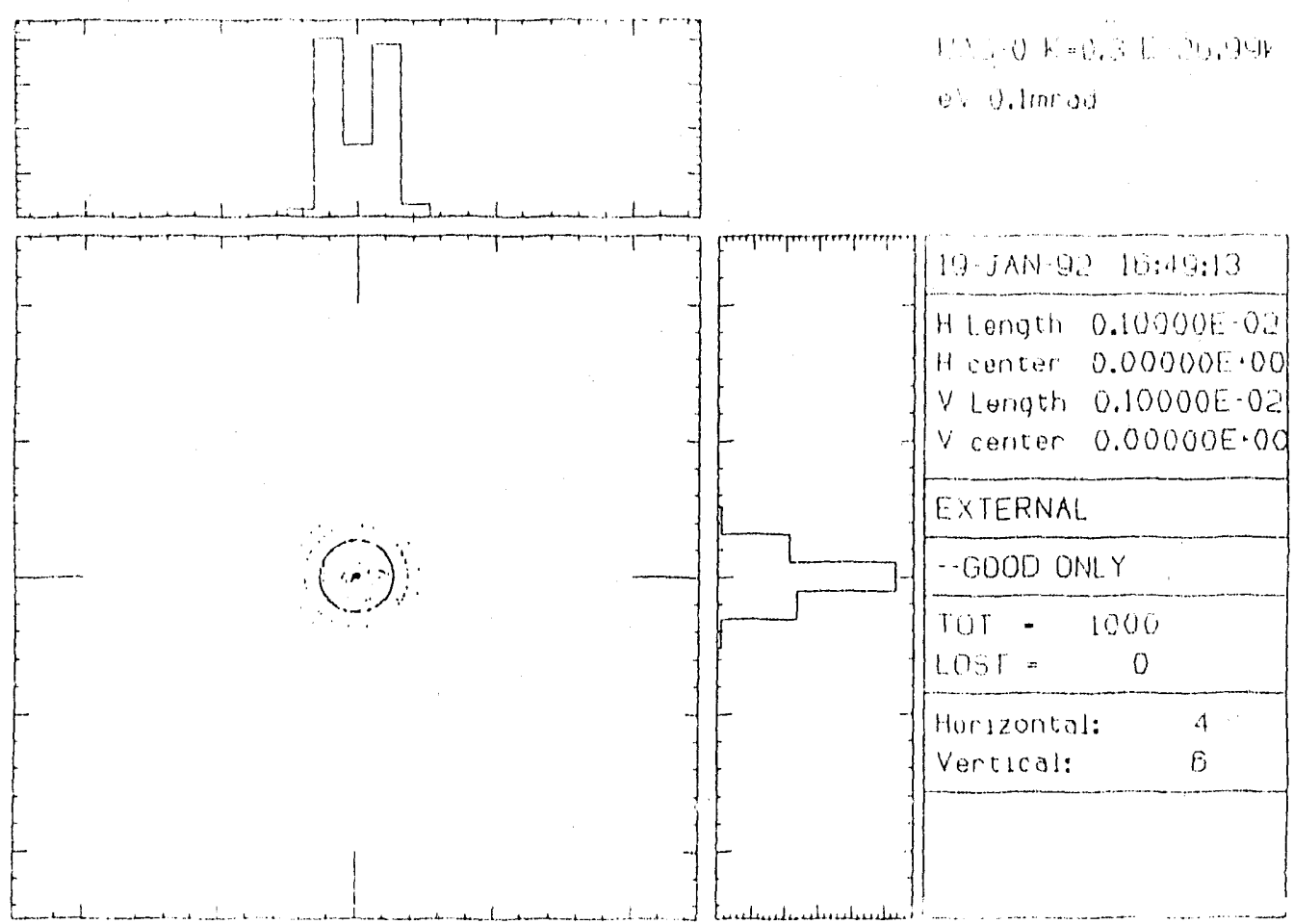

USERBISKISU AITRACY.TESTIBEGIN.0AT:25
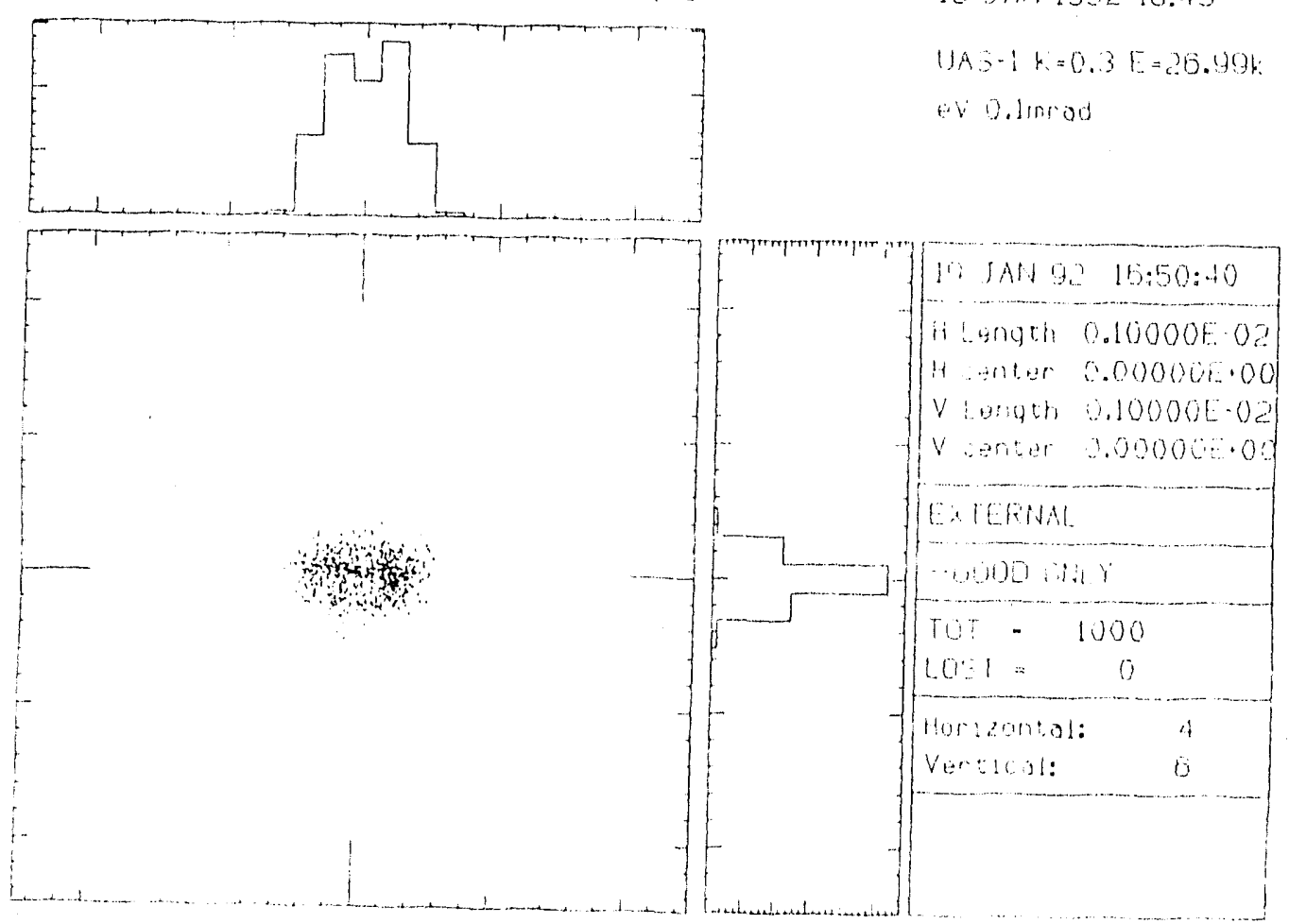

Fig. UA-56. $\mathrm{K}=0.3$, second harmonic.
$1 \because J A N 144,113: 46$

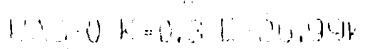

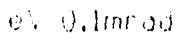

19.JAN.1902 16:49

$13.3-14=0.3 E=26.49 k$

ti $0 . \ln r o d$

I. JAN 9.2 15:50:40

if bengeh 0.16000F. 0?

H. onter a.000006.00

$V$ antath $0.10000 E-0 a$

$V$ antar $\quad 200000000$

EATERNA

-

TOT - 1000

Hor acontal:

Ver:elosle 
LSEFACEK.SLALTRAQY.TESTJBEGIN.DAT:22
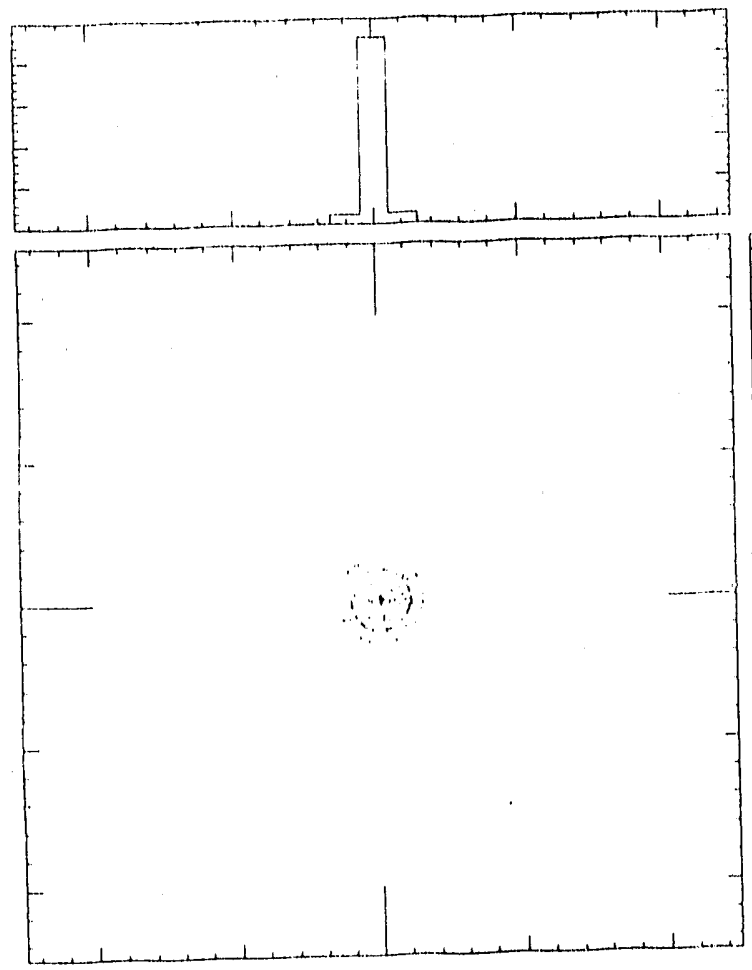

USEF.SISK:ISLAI.TRALY.TESTIBEGIN.DAT:23
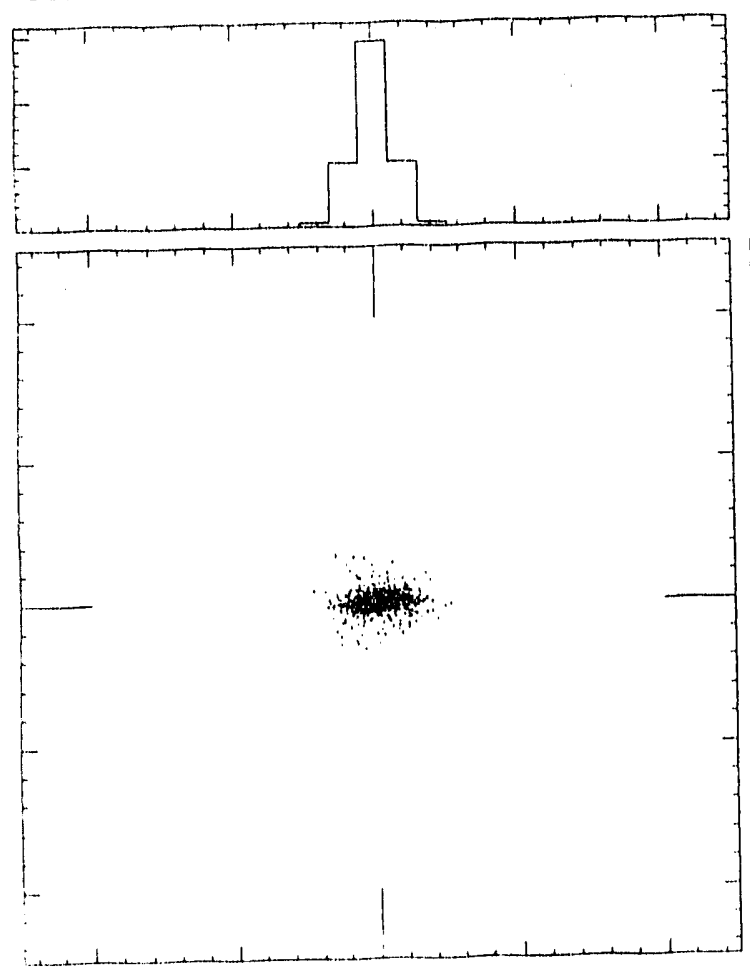

Fig. UA-57. $\quad \mathrm{K}=0.3$, third harmonic.

EXTE:RNAL.

19.5AN-1 19? 16:27

UAS-0 K=0.3E=41.49k.

$\theta \vee 0.07 \mathrm{mrad}$

$19-5$ AN-0.2 16:37:42

H Lengeh $0.10000 E-02$

H center 0.00000E-00

$\checkmark$ Lenuth $0.10000 E-02$

$v$ cencer $0.00000 E^{\circ}(0)$

- Bo(1)! Ond. Y

TOT $=1000$

LOST $=0$

Horlzontol: 4

Vertical: $\quad 6$

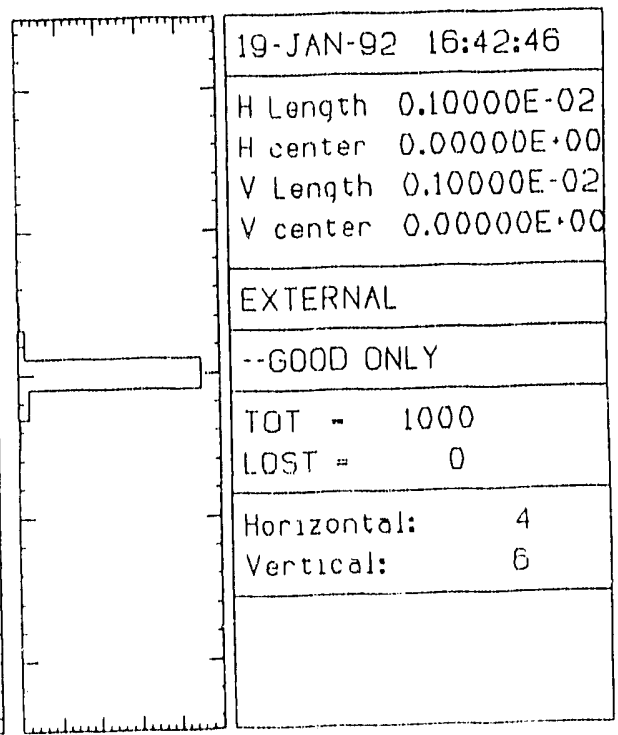

UAS- $1 K=0.3 E=40.49 K$

eV $0.07 \mathrm{mrod}$

19-JAN-1992 16:41 
APS WIGGLER A

\section{RAY-TRACING RESULTS}


-

c 

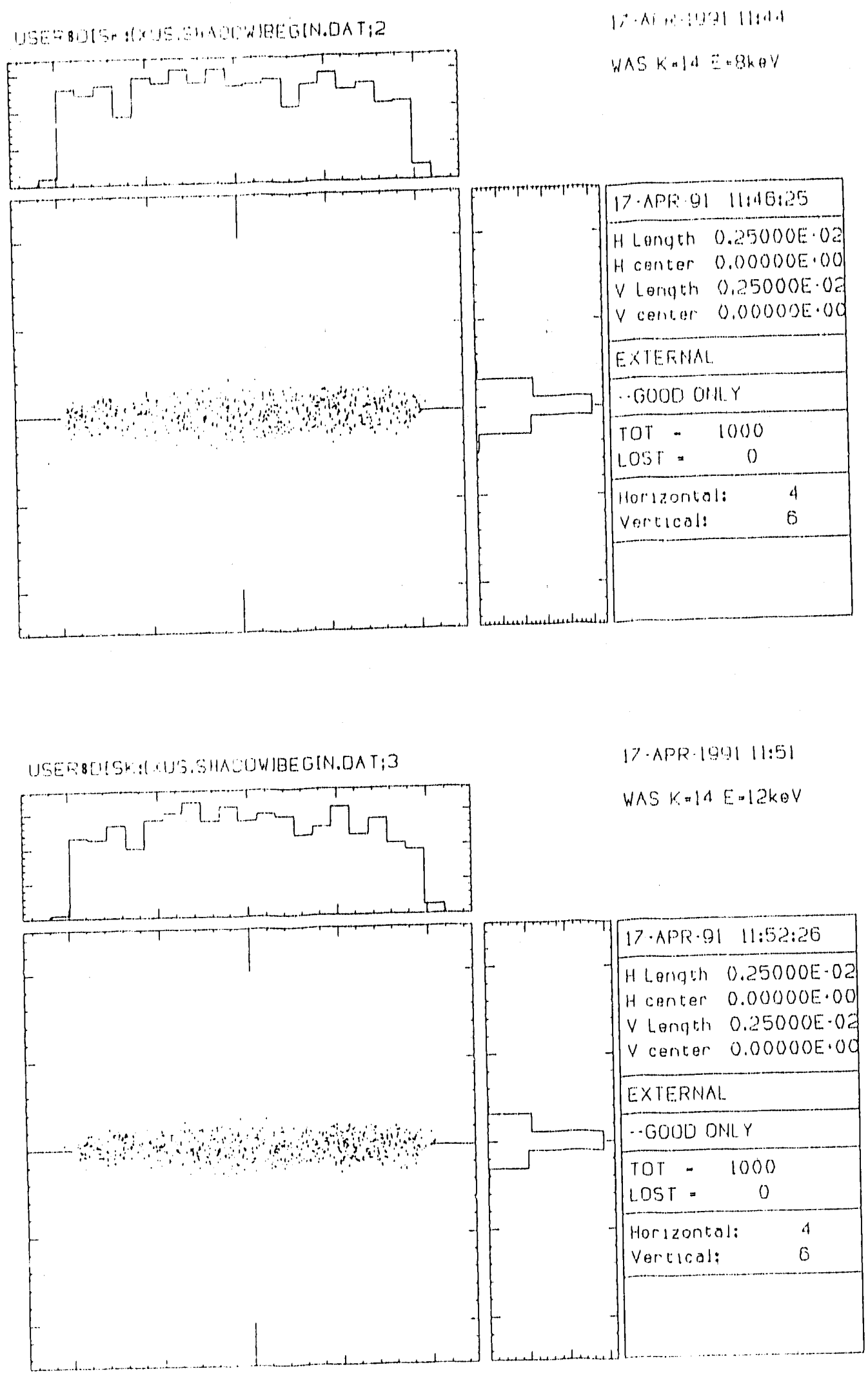

Fig. WA-1. Wiggler A. Top: $h v=8 \mathrm{keV}$, bottom: $h v=12 \mathrm{keV}$. The limits for each plot are $2.5 \mathrm{mrad} \times 2.5 \mathrm{mrad}$. 


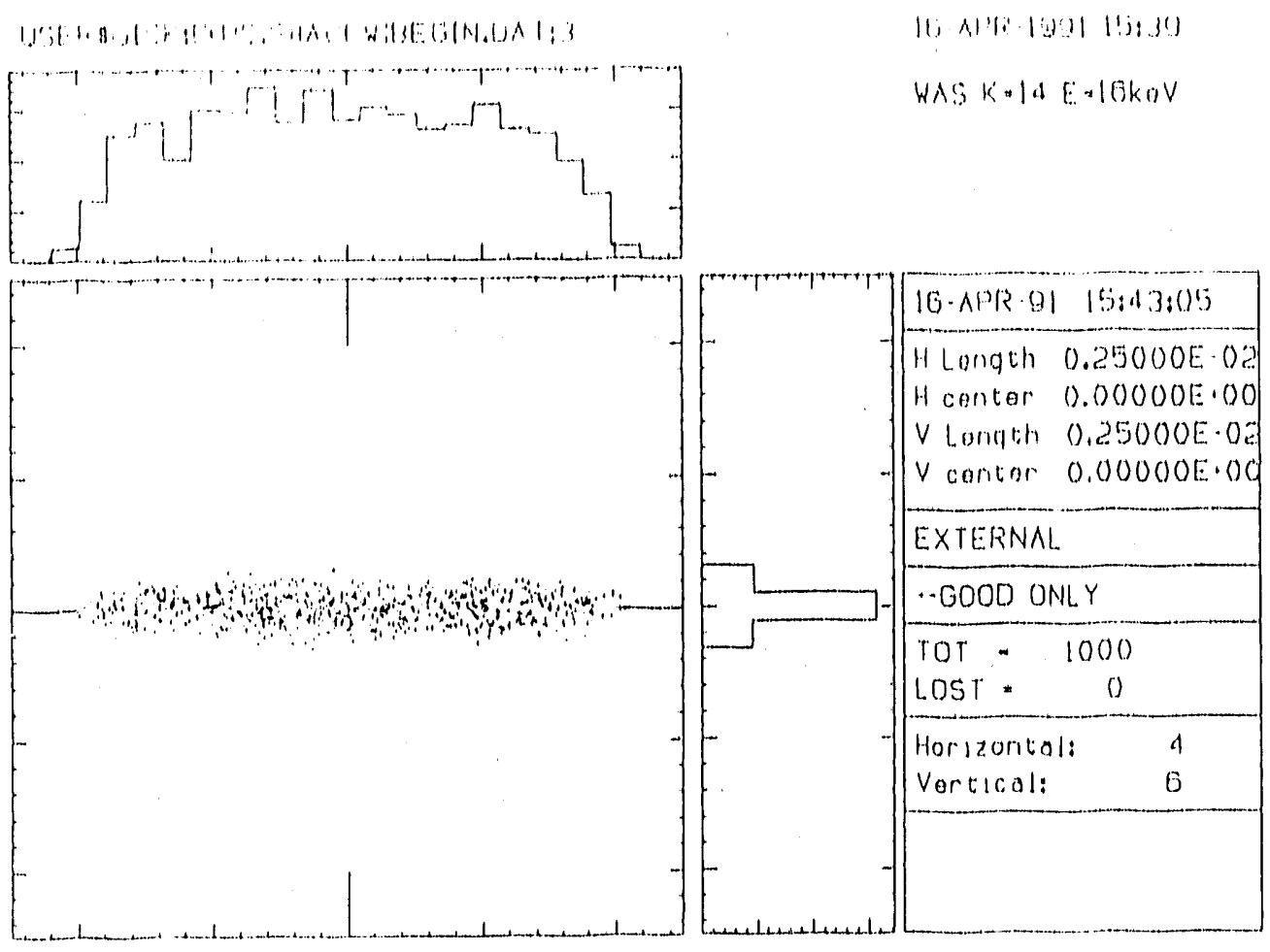

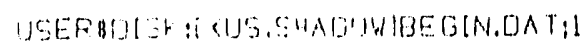
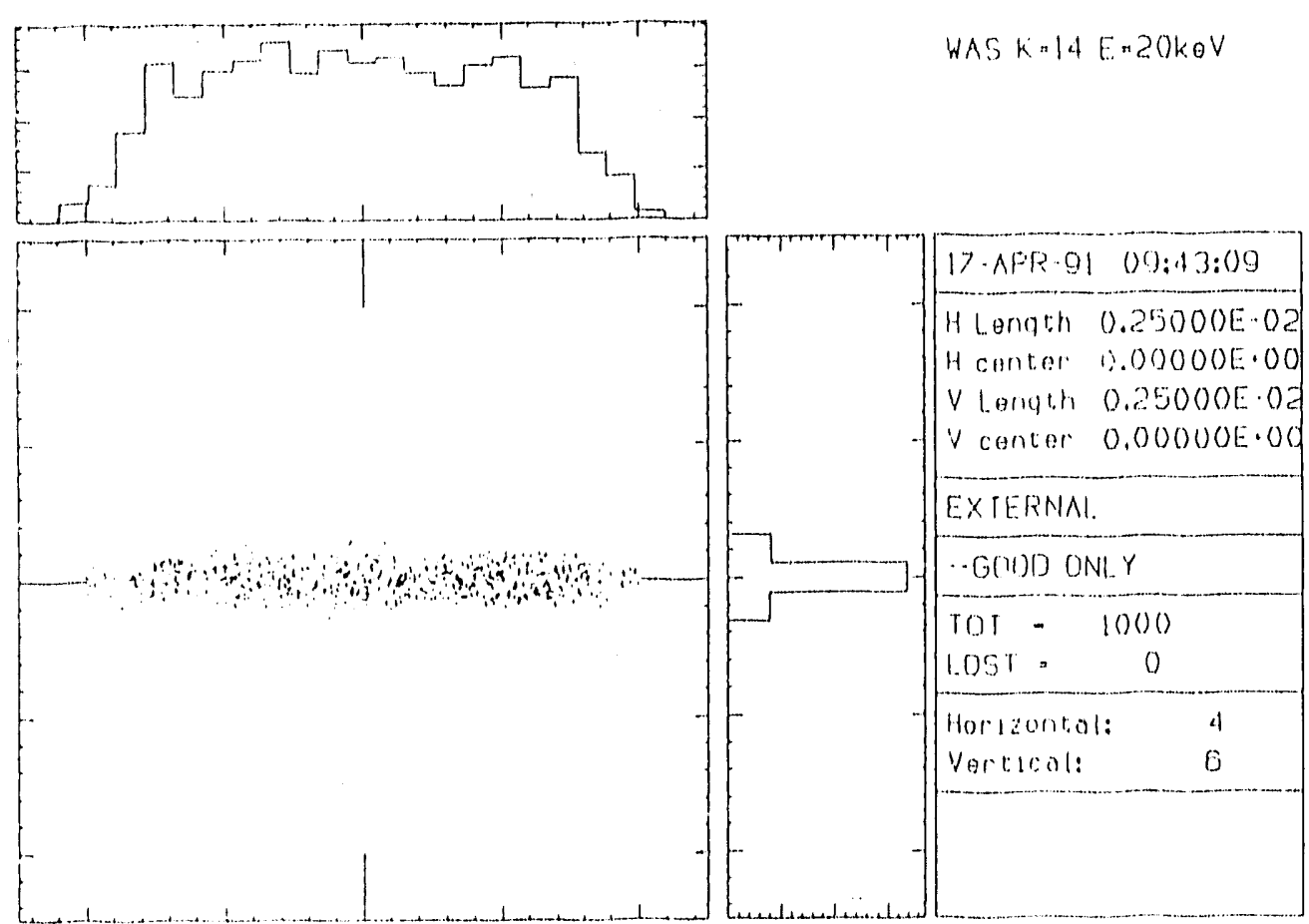

17.APF.1991 09:37

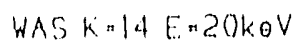

Fig. WA-2. Wiggler A. Top: hv $=16 \mathrm{keV}$, bottom: hv $=20 \mathrm{keV}$. 

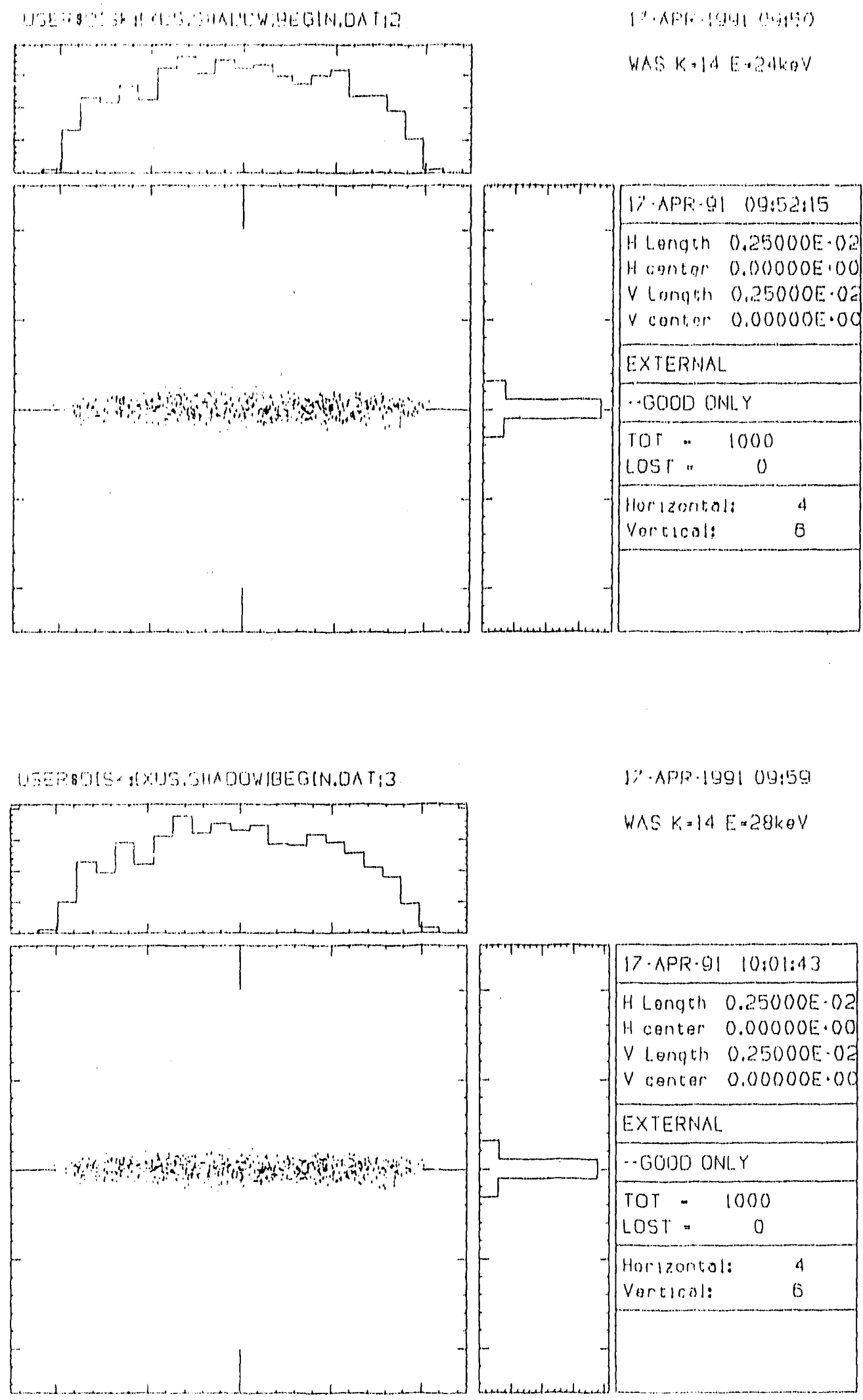

Fig. WA-3. Wiggler A. Top: hv $=24 \mathrm{keV}$, bottom: $h v=28 \mathrm{keV}$. 

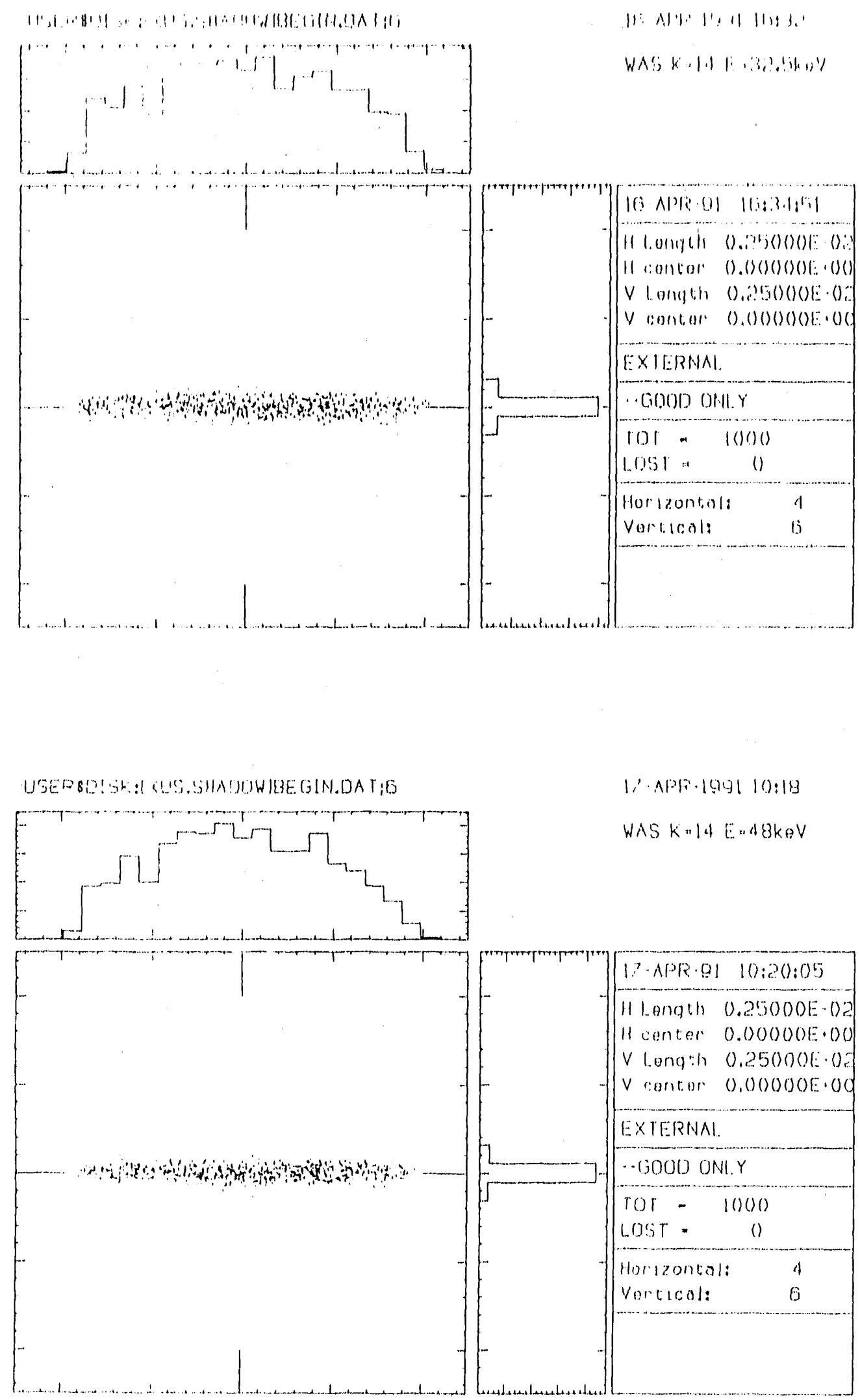

Fig. WA-4. Wiggler A. Top: $h v=32.5 \mathrm{keV}\left(=\mathrm{E}_{\mathrm{c}}\right)$, bottom: $\mathrm{hv}=48 \mathrm{keV}$. 

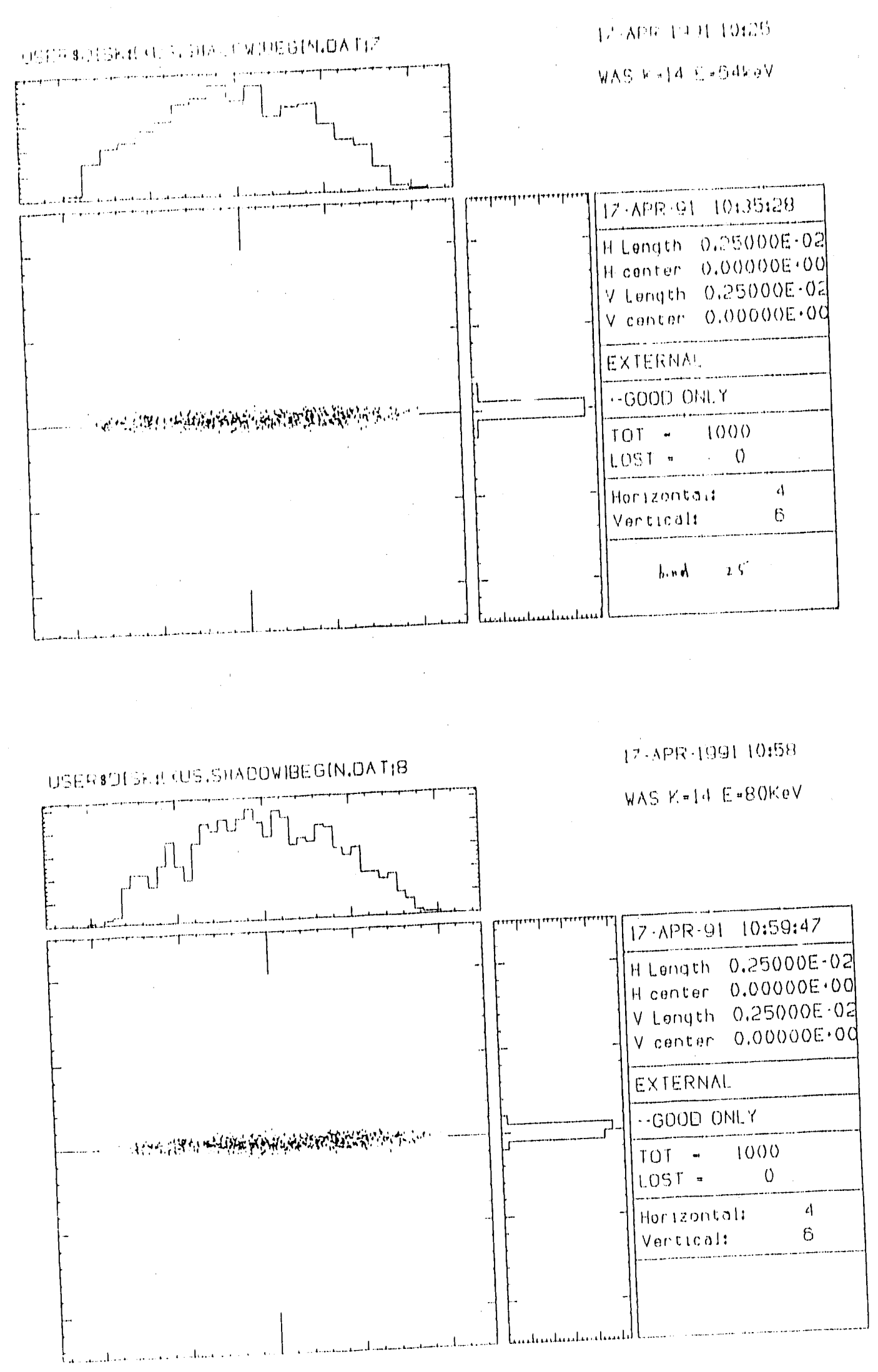

Fig. WA-5. Wiggler A. Top: hv $=64 \mathrm{keV}$, bottom: hv $=80 \mathrm{keV}$. 

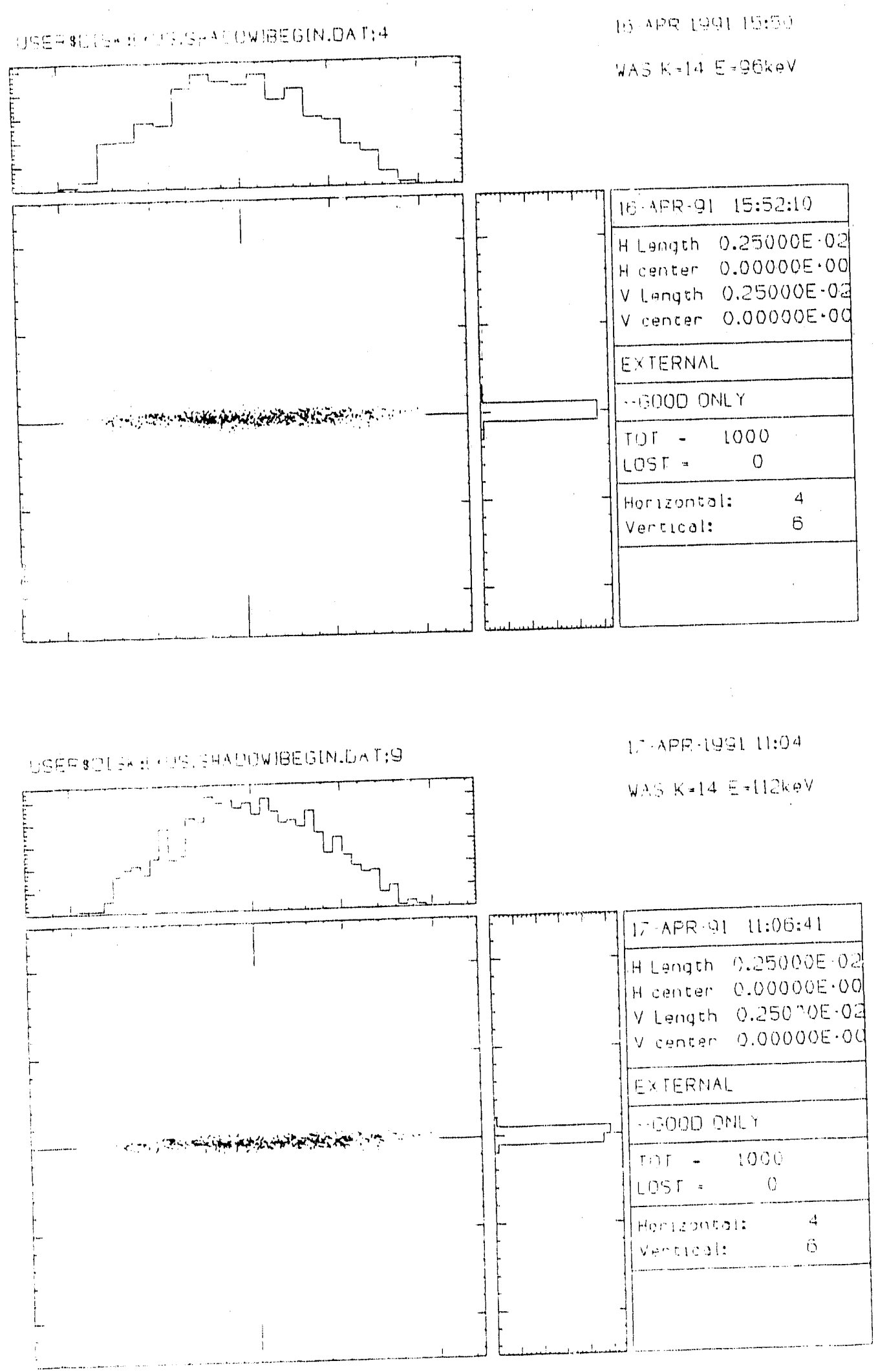

Fig. WA-6. Wiggler A. Top: $h v=96 \mathrm{keV}$, bottom: $h v=112 \mathrm{keV}$. 

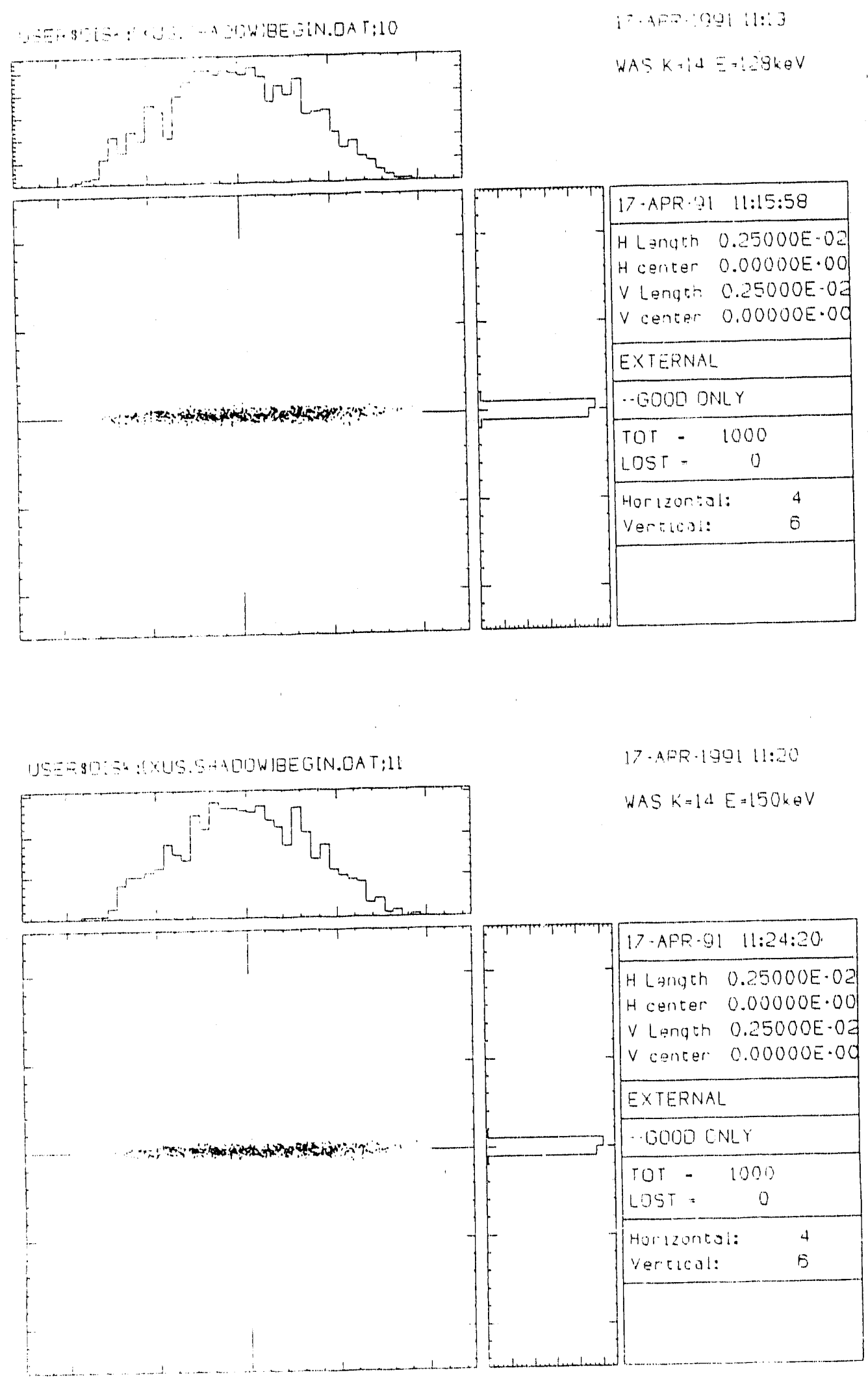

Fig. WA-7. Wiggler A. Top: hv $=128 \mathrm{keV}$, bottom: hv $=150 \mathrm{keV}$. 

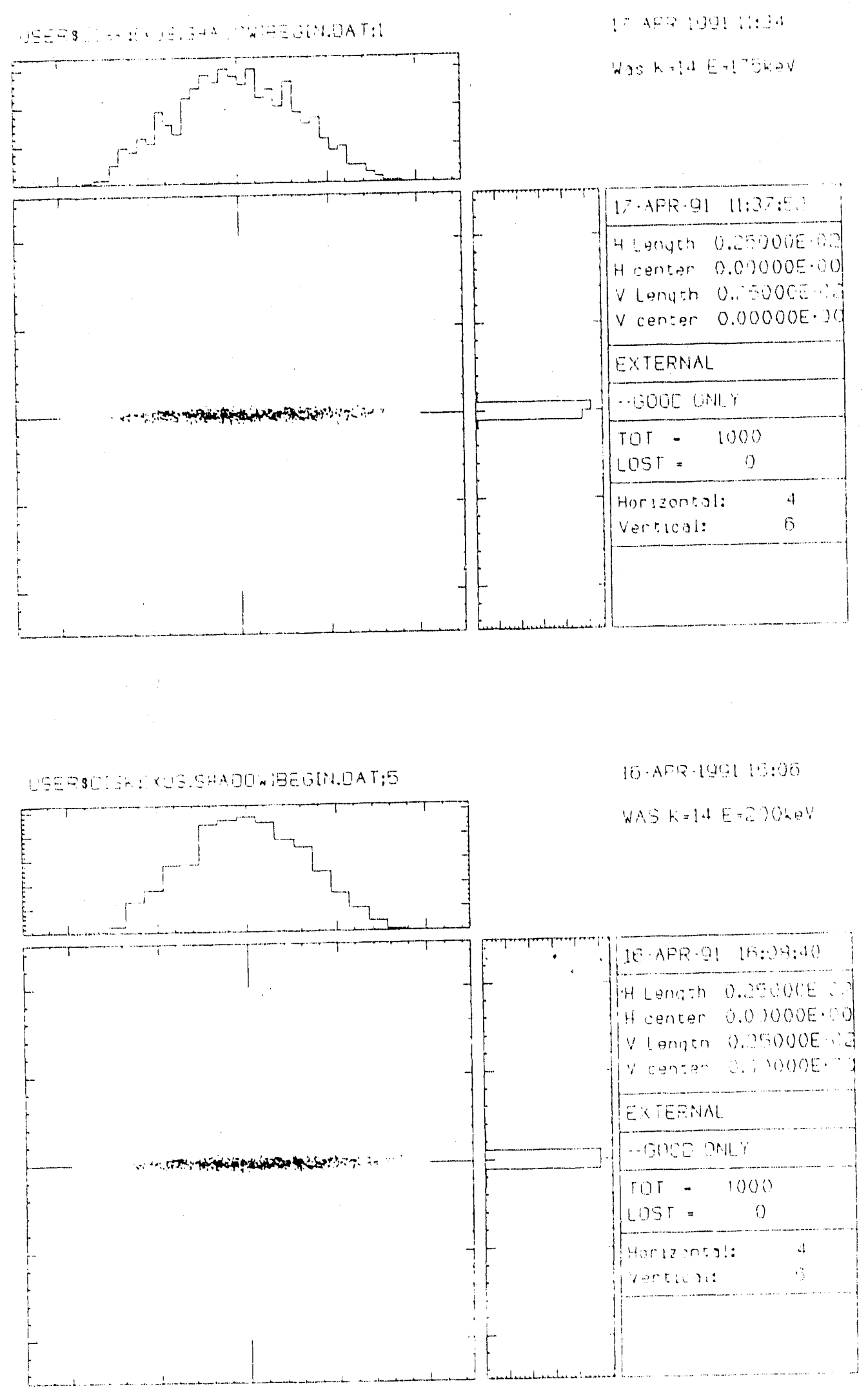

Fig. WA-8. Wiggler A. Top: $h v=175 \mathrm{keV}$, bottom: hv $=200 \mathrm{keV}$. 


\section{APS WIGGLER B}

\section{RAY-TRACING RESULTS}


-

-

c 

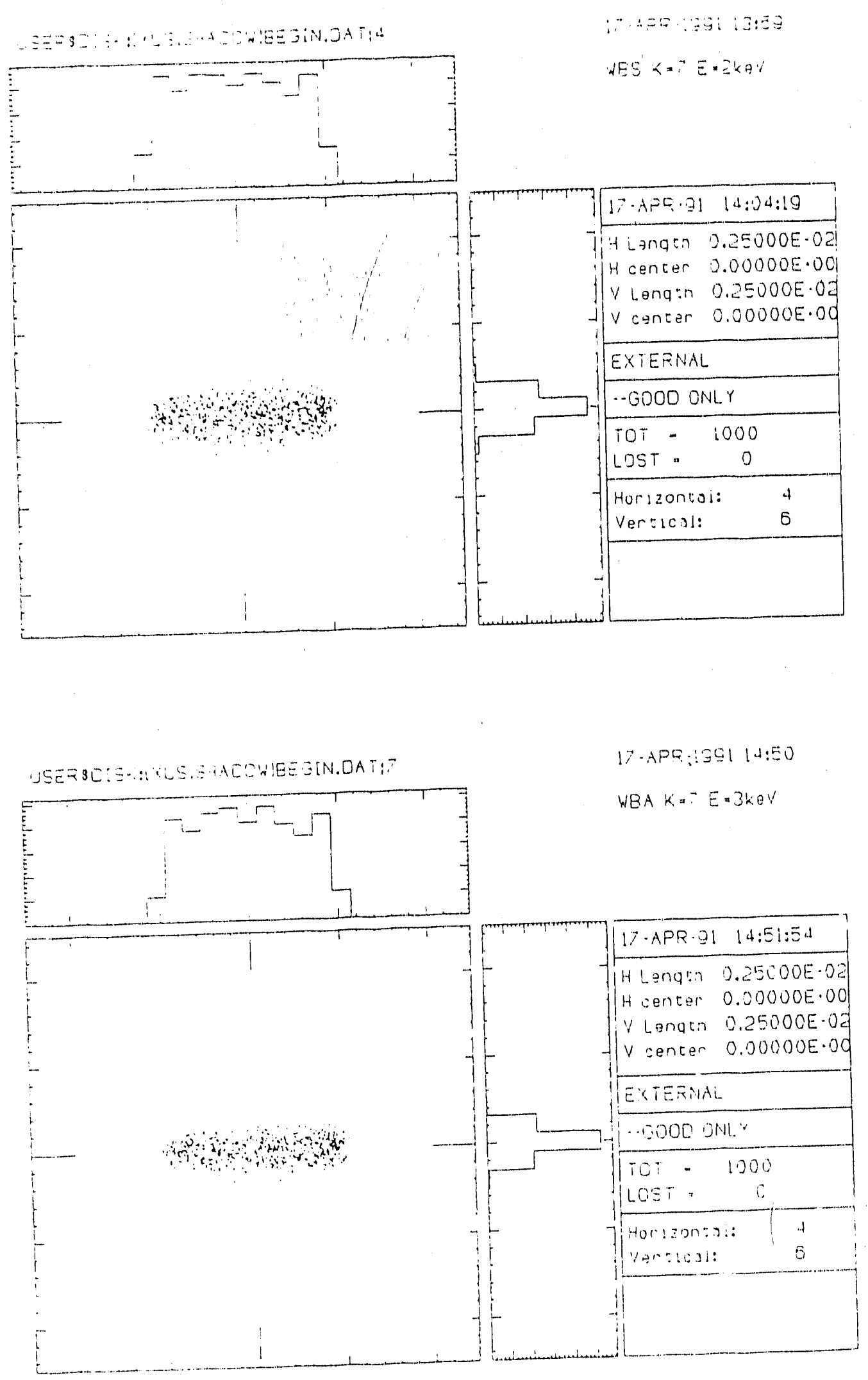

Fig. WB-1. Wiggler B. Top: $h v=2 \mathrm{keV}$, bottom: hv $=3 \mathrm{keV}$. The limits for each plot are $2.5 \mathrm{mrad} \times 2.5 \mathrm{mrad}$. 

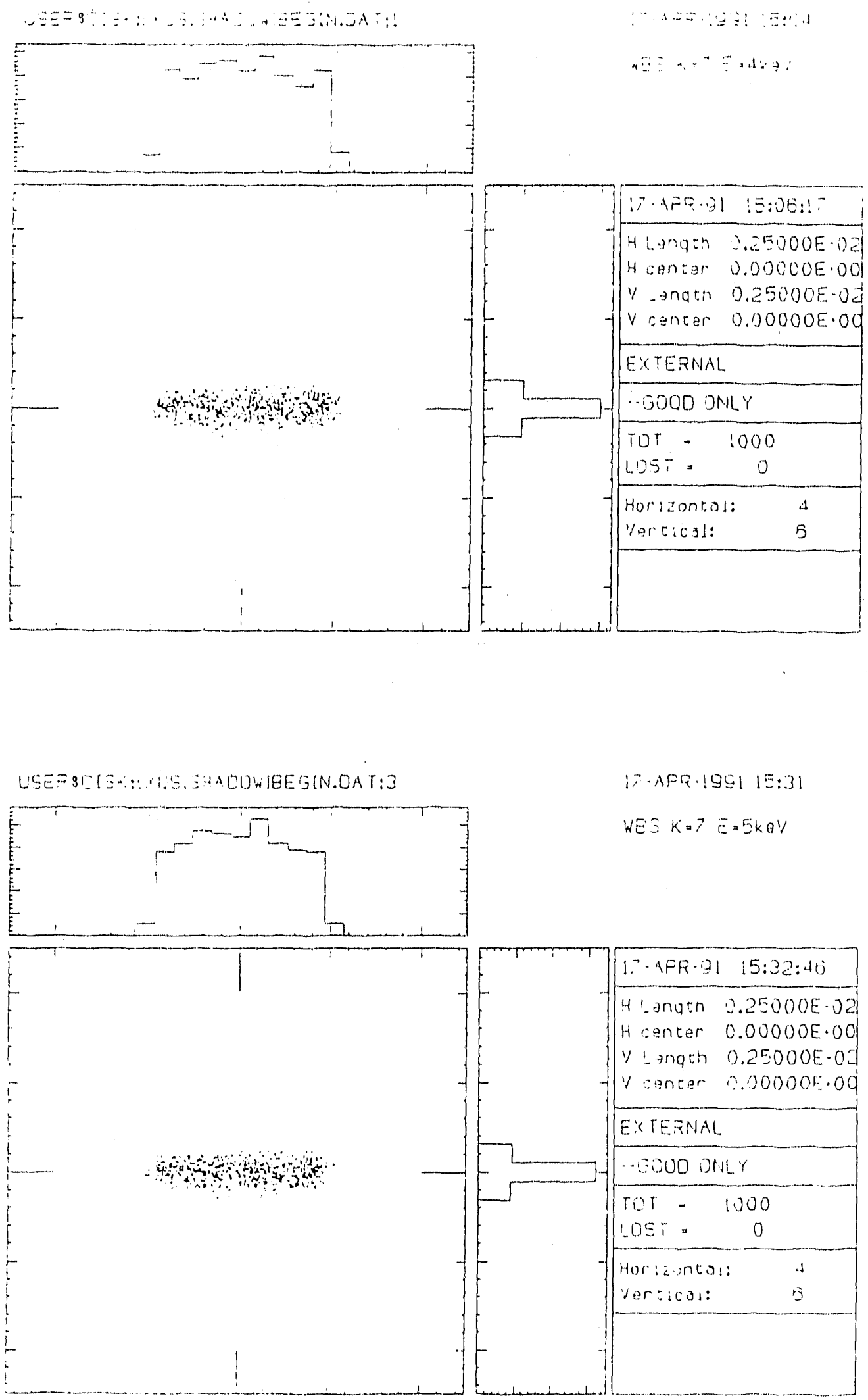

Fig. WB.2. Wiggler B. Top: hv $=4 \mathrm{keV}$, bottom: hv $=5 \mathrm{keV}$. 

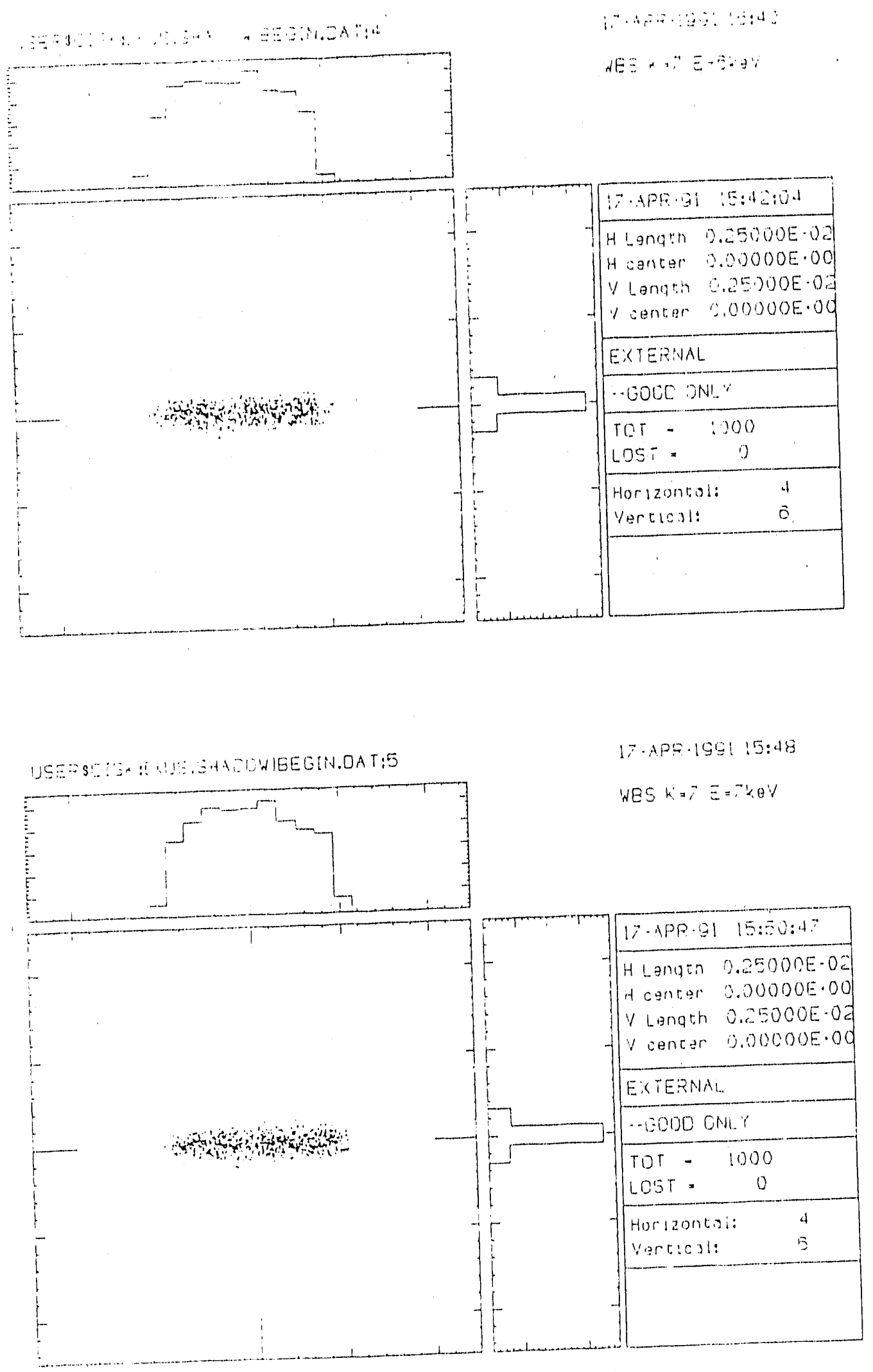

Fig. WB-3. Wiggler B. Top: $h v=6 \mathrm{keV}$, bottom: $h v=7 \mathrm{keV}$. 

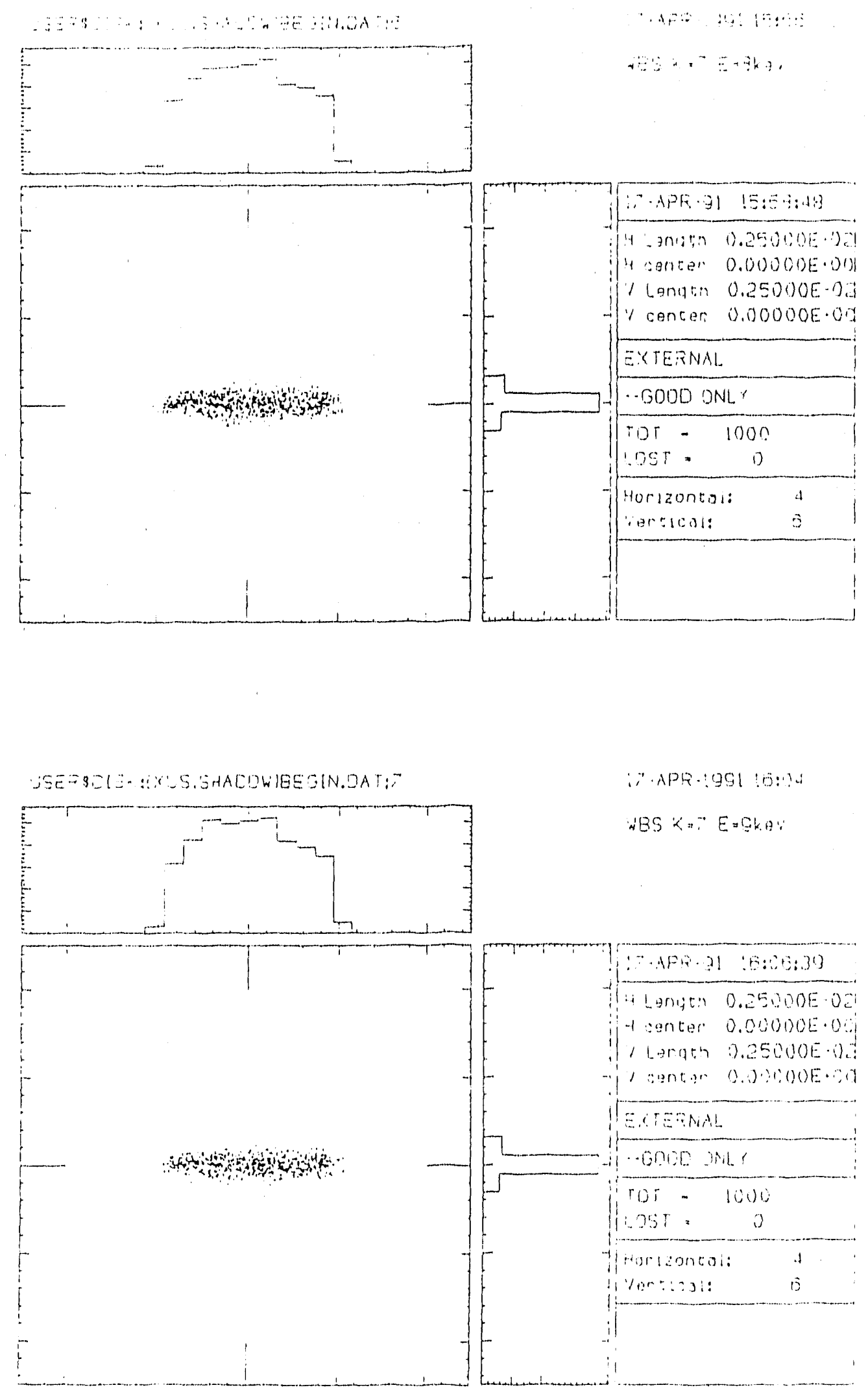

Fig. WB-4. Wiggler B. Top: hv $:=8 \mathrm{keV}$, bottom: hv $=9 \mathrm{keV}$. 


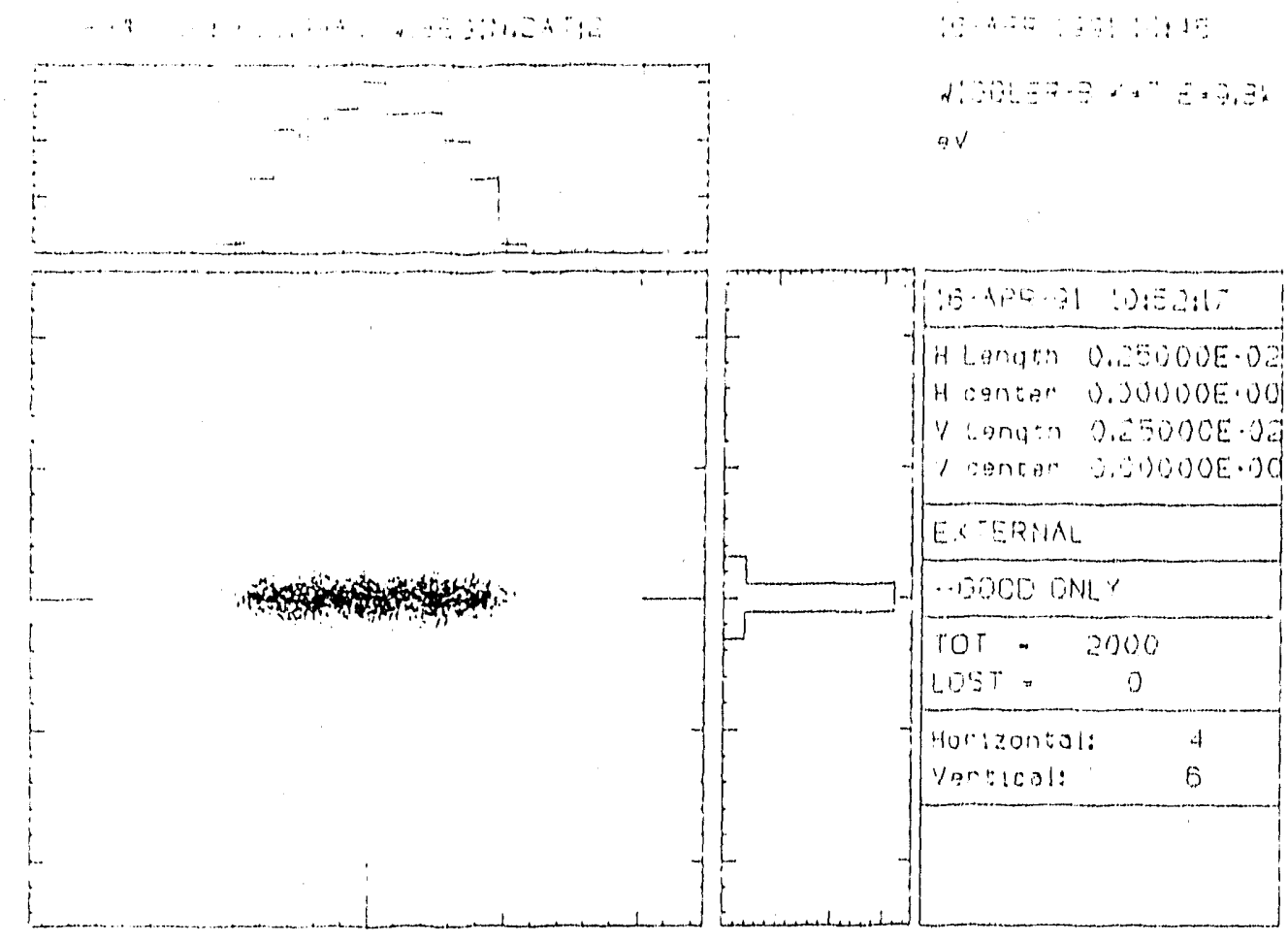

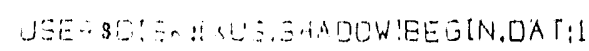

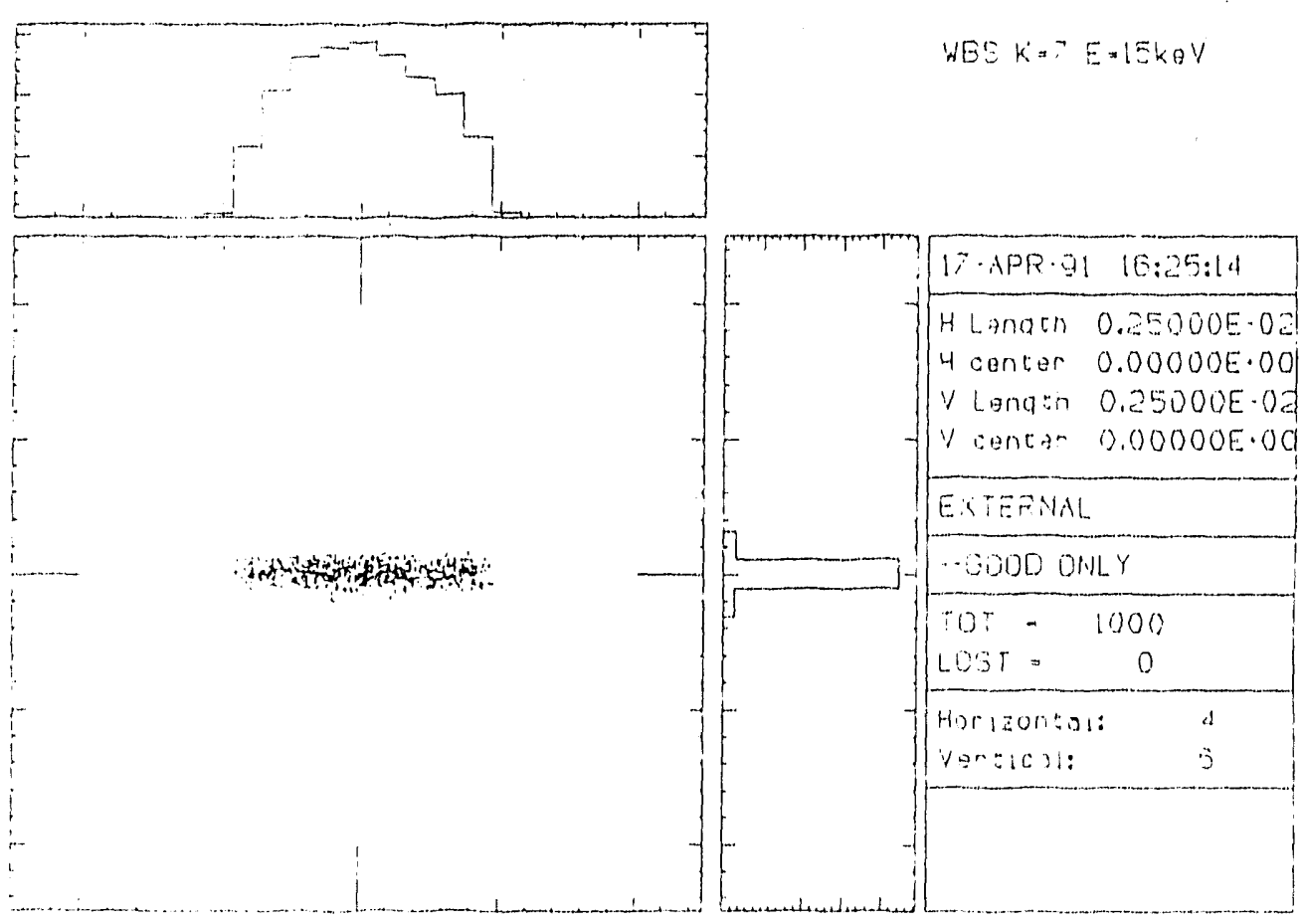

Fig. WB-5. Wiggler B. Top: hv $=9.8 \mathrm{keV}\left(=\mathrm{E}_{\mathrm{c}}\right)$, bottom: $\mathrm{hv}=15 \mathrm{keV}$. 

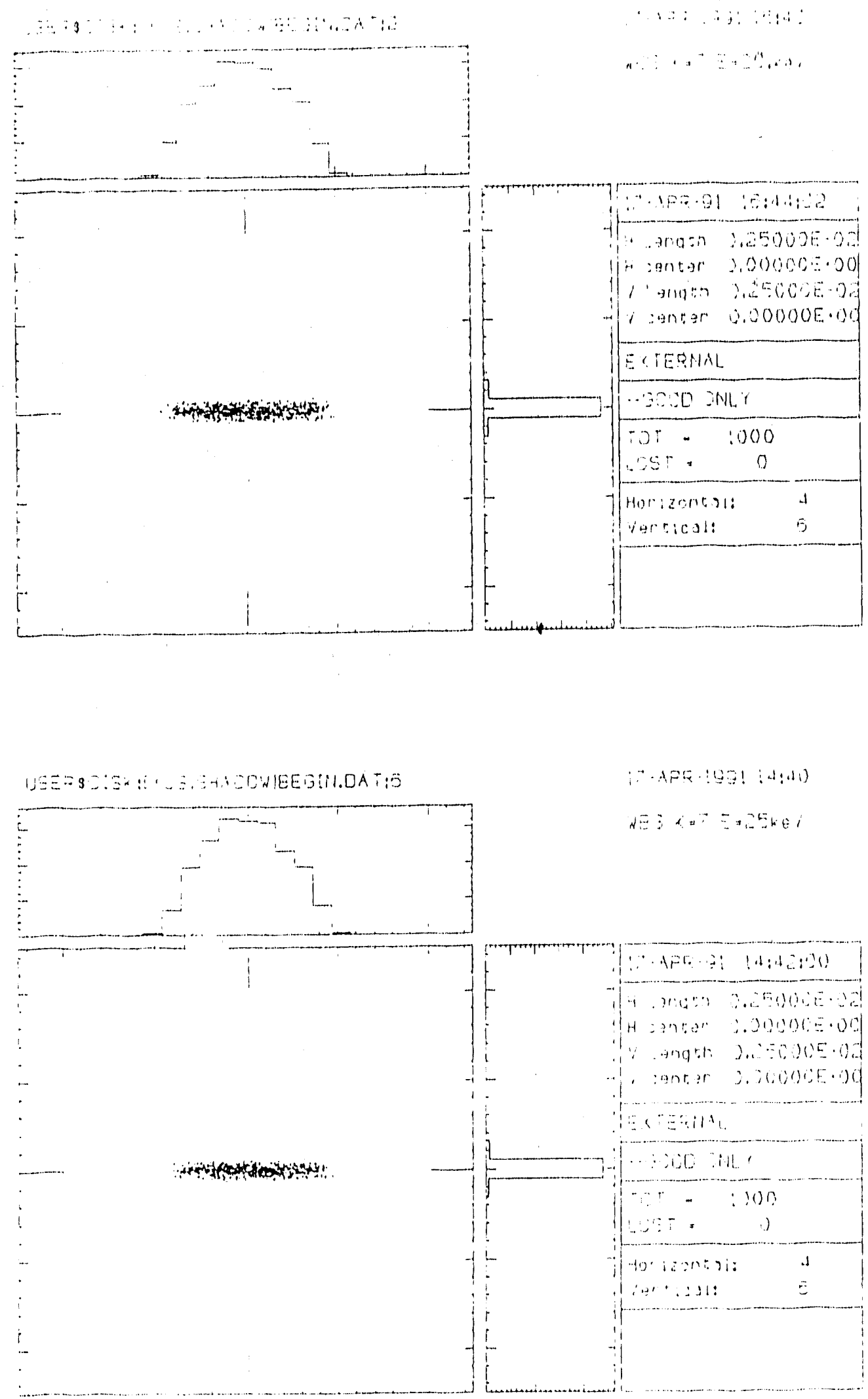

Fig. WB-6. Wiggler B. Top: hv $=20 \mathrm{keV}$, bottom: hv $=25 \mathrm{keV}$. 

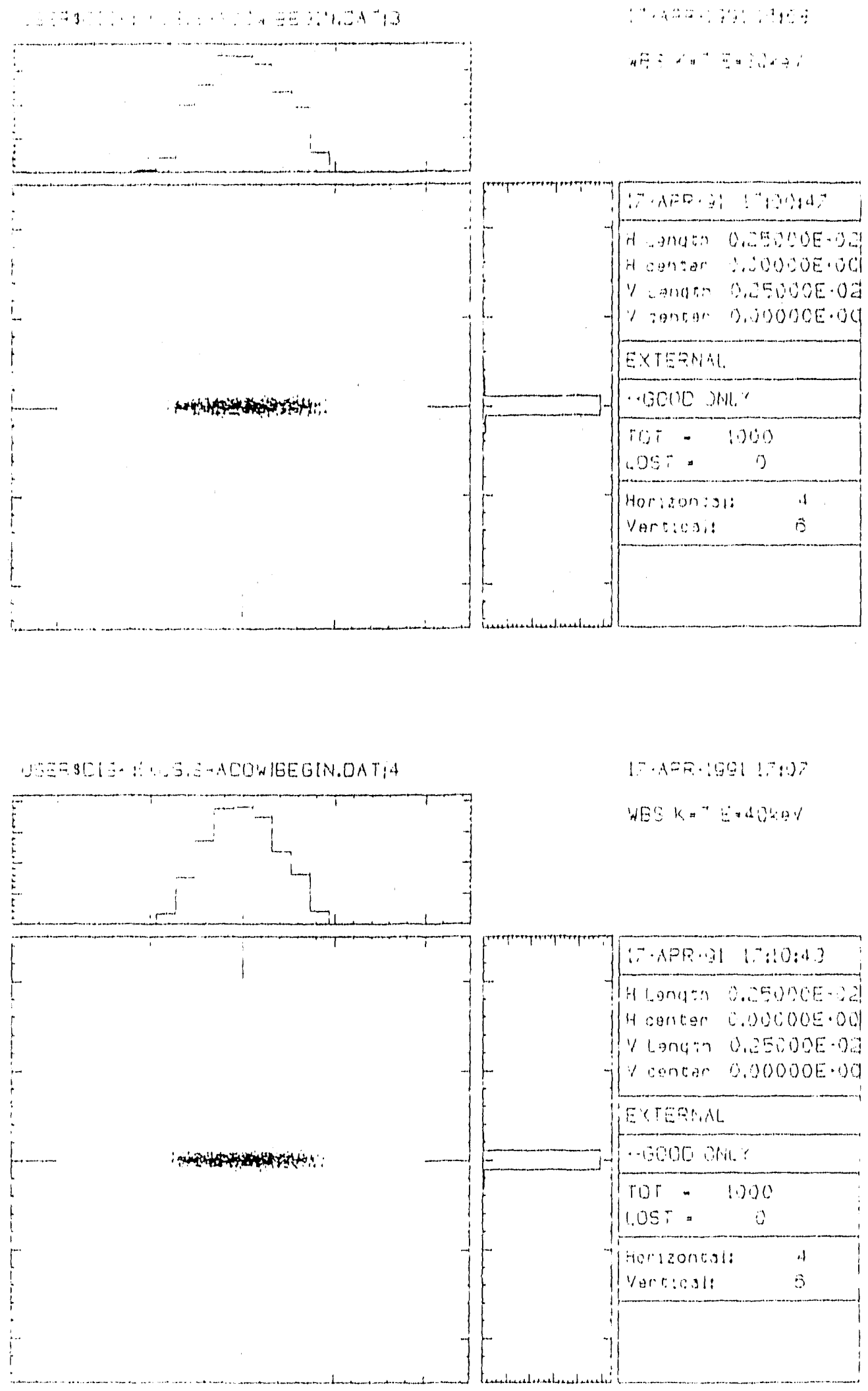

Fig. WB-7. Wiggler B. Top: hv $=30 \mathrm{keV}$, bottom: hv $=40 \mathrm{keV}$. 

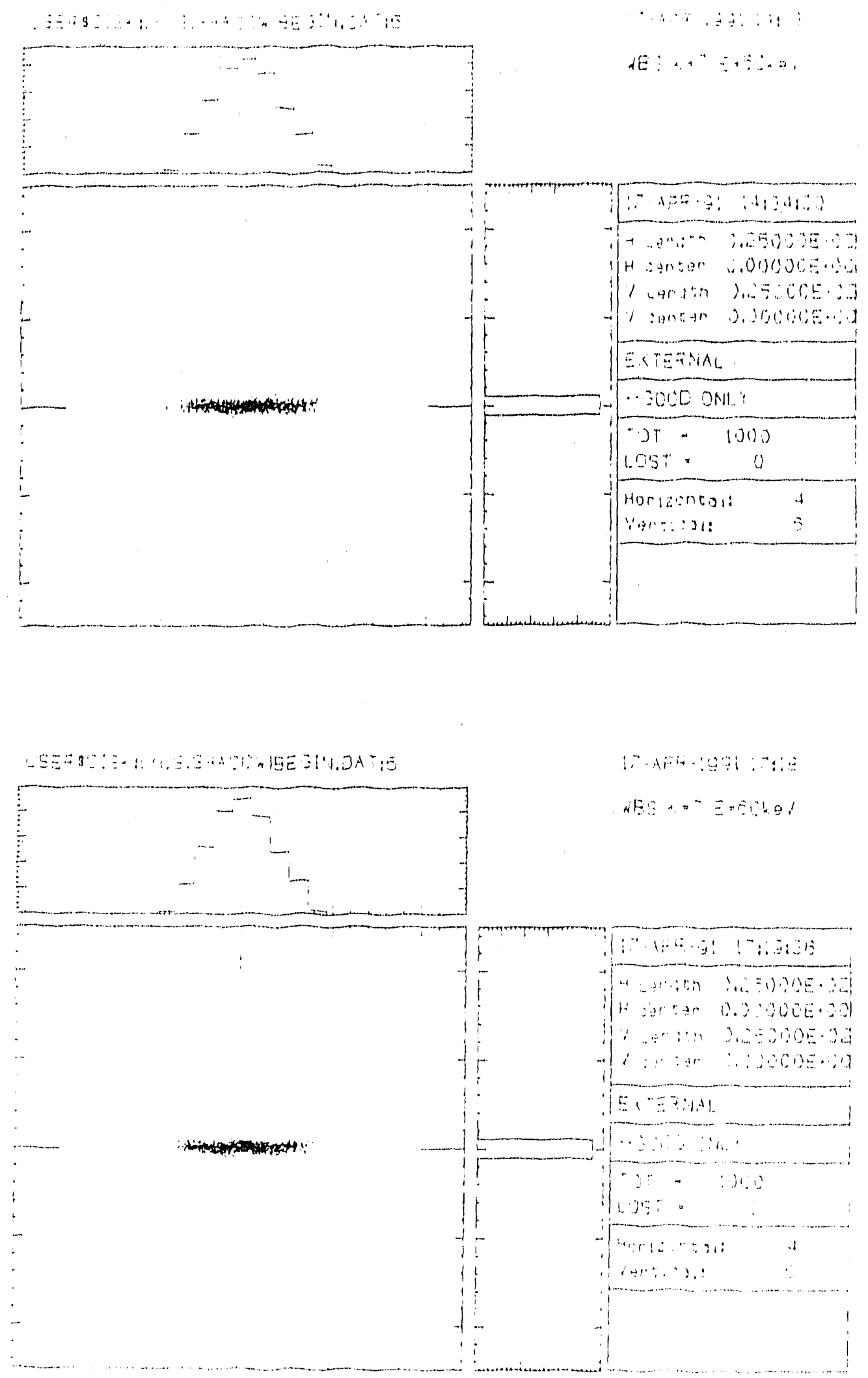

Fig. WB-8. Wiggler B. Top: hv $=50 \mathrm{keV}$, bottom: hv $=60 \mathrm{keV}$. 

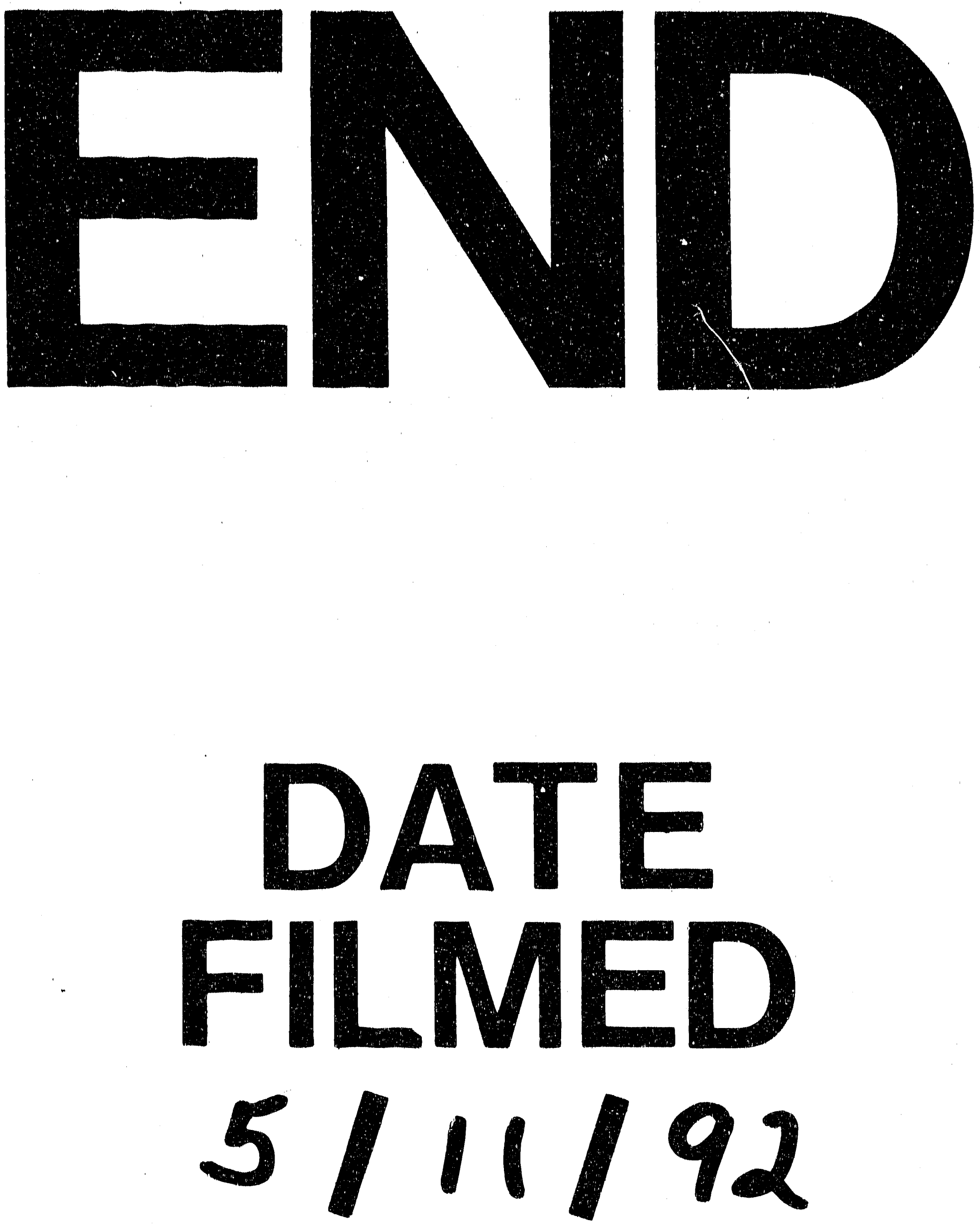